\title{
On blow up for the energy super critical defocusing nonlinear Schrödinger equations
}

\author{
Frank Merle ${ }^{1}$ - Pierre Raphaël ${ }^{2}$. \\ Igor Rodnianski ${ }^{3}$. Jeremie Szeftel ${ }^{4}$
}

Received: 15 January 2020 / Accepted: 16 August 2021 /

Published online: 15 September 2021

(C) The Author(s) 2021

Abstract We consider the energy supercritical defocusing nonlinear Schrödinger equation

$$
i \partial_{t} u+\Delta u-u|u|^{p-1}=0
$$

in dimension $d \geq 5$. In a suitable range of energy supercritical parameters $(d, p)$, we prove the existence of $e^{\infty}$ well localized spherically symmetric initial data such that the corresponding unique strong solution blows up in finite time. Unlike other known blow up mechanisms, the singularity formation does not occur by concentration of a soliton or through a self similar solution, which are unknown in the defocusing case, but via a front mechanism. Blow up is achieved by compression for the associated hydrodynamical flow which

\ Pierre Raphaël

praphael@maths.cam.ac.uk

Frank Merle

merle@math.u-cergy.fr

Igor Rodnianski

irod@math.princeton.edu

Jeremie Szeftel

jeremie.szeftel@upmc.fr

1 AGM, CY Cergy Paris Université and IHES, Cergy Pontoise cedex, France

2 Department of Pure Mathematics and Mathematical Statistics, Cambridge, UK

3 Department of Mathematics, Princeton University, Princeton, NJ, USA

4 CNRS and Laboratoire Jacques Louis Lions, Sorbonne Université, Paris, France 
in turn produces a highly oscillatory singularity. The front blow up profile is chosen among the countable family of $e^{\infty}$ spherically symmetric self similar solutions to the compressible Euler equation whose existence and properties in a suitable range of parameters are established in the companion paper (Merle et al. in Preprint (2019)) under a non degeneracy condition which is checked numerically.

\section{Mathematics Subject Classification 35Q55}

\section{Introduction}

We consider the defocusing nonlinear Schrödinger equation

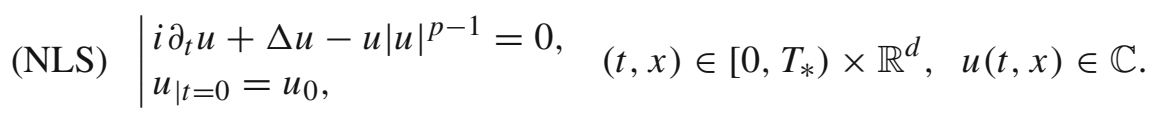

in dimension $d \geq 3$ for an integer nonlinearity $p \in 2 \mathbb{N}^{*}+1$ and address the problem of its global dynamics. We begin by giving a quick introduction to the problem and its development.

\subsection{Cauchy theory and scaling}

It is a very classical statement that smooth well localized initial data $u_{0}$ yield local in time, unique, smooth, strong solutions. For the global dynamics, two quantities conserved along the flow (1.1) are of the utmost importance:

$$
\begin{gathered}
\text { mass: } \quad M(u)=\int_{\mathbb{R}^{d}}|u(t, x)|^{2}=\int_{\mathbb{R}^{d}}\left|u_{0}(x)\right|^{2}, \\
\text { energy: } \quad E(u)=\frac{1}{2} \int_{\mathbb{R}^{d}}|\nabla u(t, x)|^{2}+\frac{1}{p+1} \int_{\mathbb{R}^{d}}|u(t, x)|^{p+1} d x=E\left(u_{0}\right) .
\end{gathered}
$$

The scaling symmetry group

$$
u_{\lambda}(t, x)=\lambda^{\frac{2}{p-1}} u\left(\lambda^{2} t, \lambda x\right), \quad \lambda>0
$$

acts on the space of solutions by leaving the critical norm invariant

$$
\int_{\mathbb{R}^{d}}\left|\nabla^{s_{c}} u_{\lambda}(t, x)\right|^{2}=\int_{\mathbb{R}^{d}}\left|\nabla^{s_{c}} u(t, x)\right|^{2} \text { for } s_{c}=\frac{d}{2}-\frac{2}{p-1} .
$$

Accordingly, the problem (1.1) can be classified as energy subcritical, critical or supercritical depending on whether the critical Sobolev exponent $s_{c}$ lies 
below, equal or above the energy exponent $s=1$. This classification also reflects the (in)/ability for the kinetic term in (1.2) to control the potential one via the Sobolev embedding $H^{1} \hookrightarrow L^{q}$.

\subsection{Classification of the dynamics}

We review the main known dynamical results which rely on the scaling classification.

Energy subcritical case. In the energy subcritical case $s_{c}<1$, the pioneering work of Ginibre-Velo [22] showed that for all $u_{0} \in H^{1}$, there exists a unique strong solution $u \in C^{0}\left(\left[0, T_{*}\right), H^{1}\right)$ to $(1.1)$ and identified the blow up criterion

$$
T_{*}<+\infty \Longrightarrow \lim _{t \uparrow T_{*}}\|u(t)\|_{H^{1}}=+\infty
$$

Conservation of energy, which is positive definite and thus controls the energy norm $H^{1}$, then immediately implies that the solution is global, $T_{*}=+\infty$. In fact, it can be shown in addition that these solutions scatter as $t \rightarrow \pm \infty$, [23]. Energy critical problem. In the energy critical case $s_{c}=1$, the criterion (1.3) fails and the energy density could concentrate. For the data with a small critical norm, Strichartz estimates allow one to rule out such a scenario, [10]. The large data critical problem has been an arena of an intensive and remarkable work in the last 20 years.

For large spherically symmetric data in dimensions $d=3,4$, the energy concentration mechanism was ruled out by Bourgain [7] and Grillakis [25] via a localized Morawetz estimate. In Bourgain's work, a new induction on energy argument led to the statements of both the global existence and scattering. These results were extended to higher dimensions by Tao [60].

The interaction Morawetz estimate, introduced in [11], led to a breakthrough on the global existence and scattering for general solutions without symmetry, first in $d=3$, [11], then in $d=4$, [55], and $d \geq 5$, [65].

A new approach was introduced in Kenig-Merle [30] in which, if there exists one global non-scattering solution, then using the concentration compactness profile decomposition [2,46], one extracts a minimal blow up solution and proves that up to renormalization, such a minimal element must behave like a soliton. The existence of such objects is ruled out using the defocusing nature of the nonlinearity, which is directly related to the non existence of solitons for defocusing models.

In all of these large data arguments, the a priori bound on the critical norm provided by the conservation of energy plays a fundamental role. Let us note that in the energy critical focusing setting, the concentration of the critical 
norm is known to be possible via type II (non self similar) blow up with soliton profile, see e.g. [34,41,51-54].

Energy supercritical problem. In the energy supercritical range $s_{c}>1$, local in time unique strong solutions can be constructed in the critical Sobolev space $H^{s_{c}},[10,32]$. Kenig-Merle's approach, [31,32], gives a blow up criterion

$$
T_{*}<+\infty \Longrightarrow \limsup _{t \uparrow T_{*}}\|u(t, \cdot)\|_{H^{s_{c}}}=+\infty,
$$

but the question of whether this actually happens for any solution remained completely open. On the other hand, the main difficulty in proving that $T_{*}=\infty$ for all solutions is that there are no a priori bounds at the scaling level of regularity $H^{s_{c}}$.

\subsection{Qualitative behavior for supercritical models}

The question of global existence or blow up for energy supercritical models is a fundamental open problem in many nonlinear settings, both focusing and defocusing. For focusing problems, the existence of finite energy type I (self similar) blow up solutions is known in various instances, see e.g. [15, 19,36, 38], and solitons have been proved to be admissible blow up profiles in certain type II (non self-similar) blow up regimes in all three settings of heat, wave and Schrödinger equations, see e.g. [14,28,39,42,49]. There are also several examples of supercritical problems with positive definite energy (wave maps, Yang-Mills) which admit smooth self-similar profiles and thus provide explicit blow up solutions, $[5,18,58]$.

On the other hand, for defocusing problems, soliton-like solutions are known not to exist and admissible self similar solutions are expected not to exist. For a simple defocusing model like the scalar nonlinear defocusing heat equation $\partial_{t} u=\Delta u-|u|^{p-1} u$, a direct application of the maximum principle ensures that $L^{\infty} \cap H^{1}$ data yield uniformly bounded solutions which are global in time and in fact dissipate. We recall again that for the energy critical problems, blow up occurs in the focusing case, where solitons exist, and it does not in the defocusing case where solitons are known not to exist.

This collection of facts led to the belief, as explicitly conjectured by Bourgain in [6], that global existence and scattering should hold for the energy supercritical defocusing Schrödinger and wave equations. Indications of various qualitative behaviors supporting different conclusions have been provided (we give a highly incomplete list) in numerical simulations e.g. [12,50], in model problems showing blow up e.g. [61,62], in examples of global solutions e.g. $[4,33]$, in logarithmically supercritical problems e.g. [13,59,63], and in ill-posedness and norm inflation type results e.g. [1,24,35,64]. 
The behavior of solutions in other supercritical models such as the ones arising in fluid and gas dynamics is extremely interesting and not yet well understood. We will not discuss it here.

\subsection{Statement of the result}

We assert that in dimensions $5 \leq d \leq 9$ the defocusing (NLS) model (1.1) admits finite time type II (non self similar) blow up solutions arising from $e^{\infty}$ well localized initial data. The singularity formation is based neither on soliton concentration nor self similar profiles, but on a new front scenario producing a highly oscillatory blow up profile: the leading order dynamics, after renormalization, is given by a type I (self-similar) singularity formation for the compressible Euler equation. The first step of our analysis is to construct $e^{\infty}$ self-similar solutions to the compressible Euler equations in a suitable range of parameters, which is done in full details in the companion paper [43]. The proof of existence of those solutions involves a non vanishing condition for an explicit constant

$$
S_{\infty}(d, \ell) \neq 0, \quad \ell=\frac{4}{p-1},
$$

which is checked numerically in the range of $(p, d)$ considered in [43].

The main result of this paper is the following.

Theorem 1.1 (Existence of energy supercritical type II defocusing blow up) Let

$$
(d, p) \in\{(5,9),(6,5),(8,3),(9,3)\},
$$

and let the critical blow up speed be

$$
r^{*}(d, \ell)=\frac{\ell+d}{\ell+\sqrt{d}}, \quad \ell=\frac{4}{p-1} .
$$

Assume that (1.4) holds for the range (1.5) as is checked numerically ${ }^{1}$ in [43]. Then there exists a discrete sequence of blow up speeds $\left(r_{k}\right)_{k \geq 1}$ with

$$
2<r_{k}<r^{*}(d, \ell), \quad \lim _{k \rightarrow+\infty} r_{k}=r^{*}(d, \ell)
$$

\footnotetext{
1 According to Lemma 5.4 in [43], $S_{\infty}$ is the limit of a sequence $S_{k}$ satisfying $\left|S_{k}-S_{\infty}\right| \leq \frac{C}{k}$ for a constant $C>0$. The sequence $S_{k}$ is defined by iteration, and we use matlab to compute it. In the range (1.5), it suffices in practice to run the computations until $S_{99}$ to check that $S_{\infty} \neq 0$ as the sequence $S_{k}$ is almost constant for $k \geq 62$. We refer to "Appendix I" of [43] for more details.
} 
such that any $k \geq 1$, there exists a finite co-dimensional manifold of smooth initial data $u_{0} \in \cap_{m \geq 0} H^{m}\left(\mathbb{R}^{d}, \mathbb{C}\right)$ with spherical symmetry such that the corresponding solution to (1.1) blows up in finite time $0<T_{*}<+\infty$ at the center of symmetry with

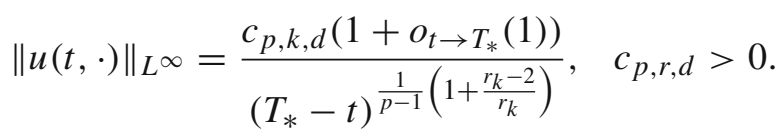

\section{Comments on the result.}

1. Hydrodynamical formulation. The heart of the proof of Theorem 1.1 is a study of (1.1) in its hydrodynamical formulation, i.e. with respect to its phase and modulus variables. The key to our analysis is the identification of an underlying compressible Euler dynamics. The latter arises as a leading order approximation of a "front" like renormalization of the original equation. In this process, the Laplace term applied to the modulus ${ }^{2}$ of the solution is treated perturbatively in the blow up regime. This is one of the key insights of the paper. The approximate Euler dynamics furnishes us with a self-similar solution, which requires very special properties and is constructed in the companion paper [43] and which, in turn, acts as a blow up profile for the original equation. The existence of these blow up profiles is directly related to the restriction on the parameters (1.5) which we discuss in comment 3 below. Let us recall that there is a long history of trying to use the hydrodynamical variables in (NLS) problems and exploit a connection with fluid mechanics, going back to Madelung's original formulation of quantum mechanics in hydrodynamical variables, [37]. Geometric optics and the hydrodynamical formulation were used to address ill-posedness and norm inflation in the defocusing Schrödinger equations, [1,24] as well as the study of the semiclassical limit [29]. There is also a recent study of vortex filaments in [3] and its dynamical use of the Hasimoto transform. The scheme of proof of Theorem 1.1 will directly apply to produce the first complete description of singularity formation for the three dimensional compressible Navier-Stokes equation in the companion paper [44].

2. Blow up profile. The blow up profile of Theorem 1.1 is more easily described in terms of the hydrodynamical variables:

$$
u(t, x)=\rho_{\operatorname{Tot}}(t, x) e^{i \phi(t, x)} .
$$

\footnotetext{
2 But not to the phase!.
} 
More precisely, we establish the decomposition

$$
\mid \begin{aligned}
& \rho_{\mathrm{Tot}}(t, x)=\frac{1}{\left(T_{*}-t\right)^{\frac{1}{p-1}}\left(1+\frac{r-2}{r}\right)}\left(\rho_{P}+\rho\right)(Z), \\
& \phi(t, x)=\frac{1}{\left(T_{*}-t\right)^{\frac{r-2}{r}}}\left(\Psi_{P}+\Psi\right)(Z),
\end{aligned} \quad Z=\frac{x}{\left(T_{*}-t\right)^{\frac{1}{r}}}
$$

and prove the local asymptotic stability

$$
\lim _{t \rightarrow T_{*}}\|\Psi\|_{L^{\infty}(Z \leq 1)}+\|\rho\|_{L^{\infty}(Z \leq 1)}=0 .
$$

Here, the blow up profile $\left(\rho_{P}, \Psi_{P}\right)$ is, after a suitable transformation, picked among the family of spherically symmetric, smooth and decaying as $Z \rightarrow+\infty$ self-similar solutions to the compressible Euler equations. The interest in selfsimilar solutions for the equations of gas dynamics goes back to the pioneering works of Guderley [26] and Sedov [57] (and references therein) who in particular considered converging motion of a compressible gas towards the center of symmetry. However, the rich amount of literature produced since then is concerned with non-smooth self-similar solutions. This is partly due to the physical motivations, e.g. interests in solutions modeling implosion or detonation waves, where self-similar rarefaction or compression is followed by a shock wave (these are self-similar solutions which contain shock discontinuities already present in the data), and, partly due to the fact that, as it turns out, global solutions with the desired behavior at infinity and at the center of symmetry are generically not $C^{\infty}$. This appears to be a fundamental feature of the self-similar Euler dynamics and, in the language of underlying acoustic geometry, means that generically such solutions are not smooth across the backward light cone (of the acoustical metric associated to the Euler profile) with the vertex at the singularity. The key of our analysis is to find those non-generic $e^{\infty}$ solutions and to discover that this regularity is an essential element in controlling suitable positivity properties of the associated linearized operator. This is at the heart of the control of the full blow up. A novel contribution of the companion paper [43] is the construction of $e^{\infty}$ spherically symmetric self-similar solutions to the compressible Euler equations with suitable behavior at infinity and at the center of symmetry for discrete values of the blow up speed parameter $r$ in the vicinity of the limiting blow up speed $r^{*}(d, \ell)$ given by (1.6).

3. Restriction on the parameters. There is nothing specific with the choice of parameters (1.5), and we refer to Remark 2.4 for a precise discussion. Two main constraints govern the restriction on the parameters. First of all, a fundamental restriction in order to make the Eulerian regime dominant is the constraint

$$
r^{*}(d, \ell)>2 \Leftrightarrow \ell<\ell_{2}(d)=d-2 \sqrt{d}
$$


which provides a non empty set of nonlinearities iff

$$
\ell_{2}(d)>0 \Leftrightarrow d \geq 5 .
$$

As a result, the case of dimensions $d=3,4$ is not amenable to our analysis at this point, and the existence of blow up solutions for $d=3,4$ remains open. The second restriction concerns the existence of $e^{\infty}$ smooth blow up profiles with suitable positivity properties of the associated linearized operator, as addressed in [43], see Sect. 2.2 and Remark 2.4 for detailed statements. In particular, a non degeneracy condition $S_{\infty}(d, \ell) \neq 0$ for an explicit convergent series is required. An elementary numerical computation to check the condition in the range (1.5).

4. Behavior of Sobolev norms. The conservation of mass and energy imply a uniform $H^{1}$ bound on the solution. This can also be checked directly on the leading order representation formulas (1.8), (1.9). For higher Sobolev norms, a computation, see "Appendix D", shows that the blow up solutions of Theorem 1.1 break scaling, i.e., we can find

$$
1<\sigma<s_{c}=\frac{d}{2}-\frac{2}{p-1}
$$

such that

$$
\lim _{t \rightarrow T_{*}}\|u(t)\|_{H^{\sigma}}=+\infty,
$$

and the critical Sobolev norm $\|u(t, \cdot)\|_{H^{s_{c}}}$ blows up polynomially.

5. Stability of blow up. The blow up profiles of Theorem 1.1 have a finite number of instability directions. Local asymptotic stability in the interior of the backward light (acoustic) cone from the singularity relies on an abstract spectral argument for compact perturbations of maximal accretive operators. Related arguments have been used in the literature for the study of self-similar solutions both in focusing and defocusing regimes, for example $[8,16,21,45,47]$ for parabolic and [19] for hyperbolic problems. The key to the control of the nonlinear flow in the exterior of the light cone is the propagation of certain weighted scale invariant norms. This generalizes a Lyapunov functional based approach developed in [42]. Counting the precise number of instability directions is an independent problem, disconnected to the nonlinear analysis of the blow up. A natural conjecture is that the number of unstable directions goes to infinity as $r_{k} \rightarrow r^{*}(d, \ell)$.

6. Non spherically symmetric perturbations. We expect that our analysis can be extended to prove the finite codimensional stability of singular dynamics to all perturbations, without the restriction to spherical symmetry. This remains to be done. 
7. Oscillatory behavior. The constructed solutions are smooth at the blow up time away from $x=0$ :

$$
\forall R>0, \quad \lim _{t \rightarrow T_{*}} u(t, x)=u^{*}(x) \text { in } H^{k}(|x|>R), \quad k \in \mathbb{N} .
$$

As in the cases for blow up problems in the focusing setting, see e.g. [40], the profile outside the blow up point has a universal behavior when approaching the singularity

$$
u^{*}(x)=c_{P}\left(1+o_{|x| \rightarrow 0}(1)\right) \frac{e^{i \frac{c_{\Psi}}{|x|^{r-2}}}}{|x|^{\frac{2(r-1)}{p-1}}}, \quad c_{P} \neq 0 .
$$

What is unusual, and together with potential non-genericity perhaps responsible for difficulties in numerical detection of the blow up phenomena, is the highly oscillatory behavior. This appears to be a deep consequence of the structure of the self-similar solution to the compressible Euler equation and the coupling of phase and modulus variable in the blow up regime, generating an anomalous Euler scaling. The heart of our analysis is to show that after passing to the suitable renormalized variables provided by the front, the highly oscillatory behavior (1.12) becomes regular near the singularity and can be controlled with the monotonicity estimates of energy type, without appealing to Fourier analysis.

8. On the role of the defocusing nonlinearity. The existence of self similar solutions to the energy supercritical (NLS) decaying at infinity is expected to hold in the focusing case, like for the heat equation [15]. In the defocusing case, such solutions are easily ruled out for the heat equation using the maximum principle, and their non existence is an open problem for the defocusing NLS, we refer to [33] for further discussion in the case of the wave equation. A fundamental observation is that in a suitable range of parameters, the semiclassical Euler limit provides admissible approximate blow up profiles for the defocusing NLS. The fact that our range of parameters is energy supercritical can be seen directly on the constraint (1.10):

$$
\begin{aligned}
r^{*}(d, \ell)>2 & \Leftrightarrow \frac{4}{p-1}=\ell<d-2 \sqrt{d} \\
& \Leftrightarrow p>1+\frac{4}{d-2 \sqrt{d}}>p_{c}=1+\frac{4}{d-2} .
\end{aligned}
$$

In other words, the existence of suitable blow up profiles given by Euler $(r<$ $\left.r^{*}(d, \ell)\right)$ combined with the constraint that the Euler regime dominates $(r>2)$ forces an energy supercritical range of parameters. 
The paper is organized as follows. In Sect. 2, we present the "front" renormalization of the flow which makes the Euler dynamics dominant, and recall all necessary facts about the corresponding self similar profile built in [43]. Theorem 1.1 reduces to building a global in time non vanishing solution to the renormalized flow (2.25) written in hydrodynamical variables. In Sect. 2.4 we detail the strategy of the proof. In Sect. 3, we introduce the functional setting related to maximal accretivity (modulo a compact perturbation) of the corresponding linear operator which leads to a statement of exponential decay in a neighborhood of the light cone for the space of solutions (modulo an a priori control of a finite dimensional manifold corresponding to the unstable directions.) In Sect. 4, we describe our set of initial data and the set of bootstrap assumptions which govern the analysis. In Sects. 5, 6, 7, we close the control of weighted Sobolev norms and the associated pointwise bounds. In Sect. 8, we close the exponential decay of low Sobolev norms by relying on spectral estimates and finite speed of propagation arguments.

\section{Notations}

The bracket

$$
\langle r\rangle=\sqrt{1+r^{2}} .
$$

The weighted scalar product for a given measure $g$ :

$$
(u, v)_{g}=\int_{\mathbb{R}^{d}} u \bar{v} g d x .
$$

The integer part of $x \in \mathbb{R}$

$$
x \leq[x]<x+1, \quad[x] \in \mathbb{Z} .
$$

The infinitesimal generator of dilations

$$
\Lambda=y \cdot \nabla \text {. }
$$

\section{Front renormalization, blow up profile and strategy of the proof}

In this section we introduce the hydrodynamical variables to study (1.1) and the associated renormalization procedure which makes the compressible Euler structure dominant. We collect from [43] the main facts about the existence of smooth spherically symmetric self-similar solutions to the compressible Euler equations which will serve as blow up profiles. 


\subsection{Hydrodynamical formulation and front renormalization}

We begin by establishing a link between the Eq. (1.1) and the compressible Euler equations. For non vanishing solutions, we write the equivalent hydrodynamical formulation in phase and modulus variables:

$$
u(t, x)=\sqrt{\rho}(\tilde{t}, x) e^{i \psi(\tilde{t}, x)}, \quad \tilde{t}=2 t .
$$

Equation (1.1) becomes a system

$$
\mid \begin{aligned}
& \partial_{\tilde{t}} \rho+\rho \Delta \psi+\nabla \rho \cdot \nabla \psi=0, \\
& \partial_{\tilde{t}} \psi+\frac{1}{2}|\nabla \psi|^{2}+\frac{1}{2} \rho^{\frac{p-1}{2}}=\frac{1}{2} \frac{\Delta \sqrt{\rho}}{\sqrt{\rho}} .
\end{aligned}
$$

This is precisely the compressible Euler (potential flow) equations (the second equation is the Bernoulli equation) for the density $\rho$, velocity $\nabla \psi$, the classical pressure

$$
P=\frac{p-1}{2(p+1)} \rho^{\frac{p+1}{2}}
$$

and the quantum stress tensor

$$
\mathcal{Q}=\frac{1}{2} \Delta \rho I-\frac{1}{2} \frac{\nabla \rho \otimes \nabla \rho}{\rho},
$$

so that

$$
\mid \begin{aligned}
& \partial_{\tilde{t}} \rho+\operatorname{div}(\rho \cdot \nabla \psi)=0, \\
& \rho \partial_{\tilde{t}} \nabla \psi+\rho \nabla \psi \cdot \nabla \nabla \psi+\nabla P=\operatorname{div} \mathcal{Q} .
\end{aligned}
$$

Below, we will show that passing to self-similar variables, the above system admits an additional front renormalization which damps the quantum stress term and therefore possesses approximate stationary solutions which, in turn, are self-similar solutions of the classical Euler equations. For convenience, we work in slightly different variables (using density squared in place of density, for instance). The correspondence between the systems derived below and the compressible Euler equations, along the lines of (2.1), will hold at every step. The explicit identification of the final approximate system (2.11) with the corresponding system representing self-similar solutions of the Euler equations is done in "Appendix A".

The standard self-similar renormalization

$$
u(t, x)=\frac{1}{\lambda(t)^{\frac{2}{p-1}}} v(\tau, y) e^{i \gamma}, \quad y=\frac{x}{\lambda},
$$


where we freeze the scaling parameter at the self-similar scale

$$
\frac{d \tau}{d t}=\frac{1}{\lambda^{2}}, \quad y=\frac{x}{\lambda(t)}, \quad-\frac{\lambda_{\tau}}{\lambda}=\frac{1}{2},
$$

then (1.1) becomes

$$
i \partial_{\tau} v+\Delta v-\gamma_{\tau} v-i \frac{\lambda_{\tau}}{\lambda}\left(\frac{2}{p-1} v+\Lambda v\right)-v|v|^{p-1}=0 .
$$

In the defocusing case, (2.2) has no known decaying type I self similar stationary solution, or type II soliton like solutions, [42], but, it turns out, that it admits approximate front like solutions. Their existence relies on a specific phase and modulus coupling and anomalous scaling. We introduce the parameters

$$
\mid \begin{aligned}
& r=\frac{2}{1-e}, \quad 0<e<1 \\
& \mu=\frac{1}{r}=\frac{1-e}{2} \\
& \ell=\frac{4}{p-1}
\end{aligned}
$$

and claim:

Lemma 2.1 (Front renormalization of the self similar flow) Define geometric parameters

$$
-\frac{\lambda_{\tau}}{\lambda}=\frac{1}{2}, \quad \frac{b_{\tau}}{b}=-e, \quad \gamma_{\tau}=-\frac{1}{b}, \frac{d \tau}{d t}=\frac{1}{\lambda^{2}}
$$

and introduce the renormalization

$$
u(t, x)=\frac{1}{\lambda(t)^{\frac{2}{p-1}}} v(\tau, y) e^{i \gamma}, \quad y=\frac{x}{\lambda}
$$

with the phase and modulus

$$
\mid \begin{aligned}
& v=w e^{i \phi}, \\
& w(\tau, y)=\frac{1}{(\sqrt{b})^{\frac{2}{p-1}}} \rho_{\mathrm{Tot}}(\tau, Z) \in \mathbb{R}_{+}^{*}, \\
& \phi(\tau, y)=\frac{1}{b} \Psi_{\mathrm{Tot}}(\tau, Z), \\
& Z=|y| \sqrt{b},
\end{aligned}
$$

In these variables (1.1) becomes, on $\left[\tau_{0},+\infty\right)$ :

$$
\mid \begin{aligned}
& \partial_{\tau} \rho_{\mathrm{Tot}}=-\rho_{\mathrm{Tot}} \Delta \Psi_{\mathrm{Tot}}-\frac{\mu \ell(r-1)}{2} \rho_{\mathrm{Tot}}-\left(2 \partial_{Z} \Psi_{\mathrm{Tot}}+\mu Z\right) \partial_{Z} \rho_{\mathrm{Tot}}, \\
& \rho_{\mathrm{Tot}} \partial_{\tau} \Psi_{\mathrm{Tot}}=b^{2} \Delta \rho_{\mathrm{Tot}} \\
& \quad-\left[\left|\nabla \Psi_{\mathrm{Tot}}\right|^{2}+\mu(r-2) \Psi_{\mathrm{Tot}}-1+\mu \Lambda \Psi_{\mathrm{Tot}}+\rho_{\mathrm{Tot}}^{p-1}\right] \rho_{\mathrm{Tot}} .
\end{aligned}
$$


Remark 2.2 Since from (2.4) we have frozen the scaling in its selfsimilar law, the lifetime of the solution in original variables is $T_{*}=e^{-\tau_{0}}$, see also (4.2).

Proof Starting from (2.2), we define a polar decomposition

$$
v=w e^{i \phi}
$$

so that

$$
v^{\prime}=\left(w^{\prime}+i \phi^{\prime} w\right) e^{i \phi}, \quad v^{\prime \prime}=w^{\prime \prime}-\left|\phi^{\prime}\right|^{2} w+2 i \phi^{\prime} w^{\prime}+i \phi^{\prime \prime} w
$$

and

$$
\begin{aligned}
0= & i \partial_{\tau} w+\Delta w+\left(-\partial_{\tau} \phi-|\nabla \phi|^{2}-\gamma_{\tau}+\frac{\lambda_{\tau}}{\lambda} y \cdot \nabla \phi\right) w \\
& +i\left(\Delta \phi-\frac{2}{p-1} \frac{\lambda_{\tau}}{\lambda}\right) w+i\left(2 \nabla \phi-\frac{\lambda_{\tau}}{\lambda} y\right) \cdot \nabla w-w|w|^{p-1} .
\end{aligned}
$$

Separating the real and imaginary parts yields the self-similar equation (2.2):

$$
\mid \begin{aligned}
& \partial_{\tau} w=-\left(\Delta \phi+\frac{1}{p-1}\right) w-\left(2 \frac{\partial_{y} \phi}{|y|}+\frac{1}{2}\right) \Lambda w \\
& w \partial_{\tau} \phi=\Delta w+\left(-|\nabla \phi|^{2}-\gamma_{\tau}-\frac{1}{2} \Lambda \phi\right) w-w|w|^{p-1} .
\end{aligned}
$$

We now renormalize according to

$$
w(\tau, y)=\frac{1}{(\sqrt{b})^{\frac{2}{p-1}}} \rho_{\operatorname{Tot}}(\tau, Z) \in \mathbb{R}_{+}^{*}, \phi(\tau, y)=\frac{1}{b} \Psi_{\mathrm{Tot}}(\tau, Z) Z=|y| \sqrt{b}
$$

with a fixed choice of parameters in the modulation equations

$$
\frac{b_{\tau}}{b}=-e, \quad \gamma_{\tau}=-\frac{1}{b}, \quad 0<e<1
$$

which transforms (2.7) into

$$
\mid \begin{aligned}
& \partial_{\tau} \rho_{\mathrm{Tot}}=-\rho_{\mathrm{Tot}} \Delta \Psi_{\mathrm{Tot}}-\frac{e+1}{p-1} \rho_{\mathrm{Tot}}-\left(2 \partial_{Z} \Psi_{\mathrm{Tot}}+\frac{1-e}{2} Z\right) \partial_{Z} \rho_{\mathrm{Tot}}, \\
& \rho_{\mathrm{Tot}} \partial_{\tau} \Psi_{\mathrm{Tot}}=b^{2} \Delta \rho_{\mathrm{Tot}} \\
& \quad-\left[\left|\nabla \Psi_{\mathrm{Tot}}\right|^{2}+e \Psi_{\mathrm{Tot}}-1+\frac{1}{2}(1-e) \Lambda \Psi_{\mathrm{Tot}}+\rho_{\mathrm{Tot}}^{p-1}\right] \rho_{\mathrm{Tot}} .
\end{aligned}
$$


We now compute from (2.3):

$$
\left|\begin{array}{l}
\frac{\mu \ell(r-1)}{2}=\frac{2}{p-1}(1-\mu)=\frac{1+e}{p-1} \\
\mu(r-2)=1-(1-e)=e
\end{array}\right|
$$

and (2.5) is proved.

\subsection{Blow up profile and Emden transform}

We recall in this section the main results of [43].

Emden transform. A stationary solution $\left(\rho_{P}, \Psi_{P}\right)$ to $(2.5)$ in the limiting Eulerian regime $b=0$ satisfies the profile equation

$$
\mid \begin{aligned}
& \left|\nabla \Psi_{P}\right|^{2}+\rho_{P}^{p-1}+\mu(r-2) \Psi_{P}+\mu \Lambda \Psi_{P}=1 \\
& \Delta \Psi_{P}+\frac{\mu \ell(r-1)}{2}+\left(2 \partial_{Z} \Psi_{P}+\mu Z\right) \frac{\partial_{Z} \rho_{P}}{\rho_{P}}=0 .
\end{aligned}
$$

We supplement it with the boundary conditions:

$$
\mid \begin{aligned}
& \rho_{P}(0)=1, \quad \Psi_{P}(0)=0, \\
& \rho_{P}(Z) \rightarrow 0, \quad \Psi_{P}(Z) \rightarrow \frac{1}{e} \text { as } Z \rightarrow \infty
\end{aligned}
$$

We now show that the system (2.8), (2.9) is equivalent to the corresponding system of equations describing self-similar solutions of the Euler equations. We define the Emden variables:

$$
\mid \begin{aligned}
& \phi=\frac{\mu}{2} \sqrt{\ell}, \quad p-1=\frac{4}{\ell}, \\
& Q=\rho_{P}^{p-1}=\frac{1}{M^{2}}, \quad \frac{1}{M}=\phi Z \sigma, \quad \varkappa=\log Z \\
& \frac{\Psi_{P}^{\prime}}{Z}=-\frac{\mu}{2} w
\end{aligned}
$$

then (2.8) is mapped onto

$$
\mid \begin{aligned}
& (w-1) w^{\prime}+\ell \sigma \sigma^{\prime}+\left(w^{2}-r w+\ell \sigma^{2}\right)=0 \\
& \frac{\sigma}{\ell} w^{\prime}+(w-1) \sigma^{\prime}+\sigma\left[w\left(\frac{d}{\ell}+1\right)-r\right]=0
\end{aligned}
$$

or equivalently

$$
\mid \begin{aligned}
& a_{1} w^{\prime}+b_{1} \sigma^{\prime}+d_{1}=0 \\
& a_{2} w^{\prime}+b_{2} \sigma^{\prime}+d_{2}=0
\end{aligned}
$$

with

$$
\mid \begin{aligned}
& a_{1}=w-1, \quad b_{1}=\ell \sigma, \quad d_{1}=w^{2}-r w+\ell \sigma^{2} \\
& a_{2}=\frac{\sigma}{\ell}, \quad b_{2}=w-1, \quad d_{2}=\sigma\left[\left(1+\frac{d}{\ell}\right) w-r\right]
\end{aligned} .
$$


The system (2.11) is exactly the one describing spherically symmetric selfsimilar solutions to the compressible Euler equation, [57] (and the references therein). For an explicit derivation see "Appendix A". It is analyzed in [43], following pioneering work of Guderley, Sedov and others.

Let

$$
w_{e}=\frac{\ell(r-1)}{d}
$$

and the determinants

$$
\mid \begin{aligned}
& \Delta=a_{1} b_{2}-b_{1} a_{2}=(w-1)^{2}-\sigma^{2} \\
& \Delta_{1}=-b_{1} d_{2}+b_{2} d_{1}=w(w-1)(w-r)-d\left(w-w_{e}\right) \sigma^{2} \\
& \Delta_{2}=d_{2} a_{1}-d_{1} a_{2}=\frac{\sigma}{\ell}\left[(\ell+d-1) w^{2}-w(\ell+d+\ell r-r)+\ell r-\ell \sigma^{2}\right]
\end{aligned}
$$

then

$$
w^{\prime}=-\frac{\Delta_{1}}{\Delta}, \quad \sigma^{\prime}=-\frac{\Delta_{2}}{\Delta}, \quad \frac{d w}{d \sigma}=\frac{\Delta_{1}}{\Delta_{2}} .
$$

Solution curves $w=w(\sigma)$ of the above system can be examined through its phase portrait in the $(\sigma, w)$ plane.

Critical points and admissible profile. The shape of the phase portrait depends crucially on the polynomials $\Delta, \Delta_{1}, \Delta_{2}$ and the parameters $(r, d, \ell)$, and we refer to [43] for a complete description. In particular, three critical points play a distinguished role in the analysis:

- the $P_{6}$ unstable point which corresponds to a point at infinity $(\sigma=$ $+\infty, w=w_{e}$ ), which in original variables corresponds to the smooth solution coming out of the origin $Z=0$,

- the $P_{4}$ stable point ( $\sigma=0, w=0$ ) which corresponds to selfsimilar decay as $Z \rightarrow+\infty$,

- the $P_{2}$ stable point which is a solution to the triple point equation

$$
\Delta\left(P_{2}\right)=\Delta_{1}\left(P_{2}\right)=\Delta_{2}\left(P_{2}\right)=0 .
$$

A classical analysis of the phase portrait reveals that in a suitable regime of parameters, there is a unique solution coming out of $P_{6}$ with the normalization

$$
\rho_{P}(0)=1, \quad \Psi_{P}(0)=0
$$

at $Z=0$, which is also $C^{\infty}$ in the vicinity of $Z=0$, and it must be attracted into $P_{2}$. This solution can be continued beyond $P_{2}$ by gluing it to a member 
of the 1-parameter family of curves that join $P_{2}$ to the selfsimilar decay $P_{4}$ as $Z \rightarrow+\infty$.

The above procedure produces a curve which is $C^{\infty}$ everywhere except at $P_{2}$ where it generically experiences an unavoidable discontinuity of high derivatives, except for discrete values of the speed $r$. The following structural proposition on the blow up profile is proved in the companion paper [43].

Theorem 2.3 (Existence and asymptotics of a $C^{\infty}$ profile, [43]) Let

$$
(d, p) \in\{(5,9),(6,5),(8,3),(9,3)\}
$$

and recall (1.6). Then there exists a sequence $\left(r_{k}\right)_{k \geq 1}$ with

$$
\lim _{k \rightarrow \infty} r_{k}=r^{*}(d, \ell), \quad r_{k}<r^{*}(d, \ell)
$$

such that for all $k \geq 1$, the following holds:

1. Existence of a smooth profile at the origin: the unique radially symmetric solution to (2.8) with Cauchy data at the origin (2.9) reaches in finite time $Z_{2}>0$ the point $P_{2}$.

2. Passing through $P_{2}$ : the solution passes through $P_{2}$ with $C^{\infty}$ regularity.

3. Large $Z$ asymptotic: the solution admits the asymptotics as $Z \rightarrow+\infty$ :

$$
\mid \begin{aligned}
& w(Z)=\frac{c_{w}}{Z^{r}}\left(1+O\left(\frac{1}{Z^{r}}\right)\right) \\
& \sigma(Z)=\frac{c_{\sigma}}{Z^{r}}\left(1+O\left(\frac{1}{Z^{r}}\right)\right)
\end{aligned}
$$

or equivalently

$$
\mid \begin{aligned}
& Q(Z)=\rho_{P}^{p-1}(Z)=\frac{c_{P}^{p-1}}{Z^{2(r-1)}}\left(1+O\left(\frac{1}{Z^{r}}\right)\right) \\
& \Psi_{P}(Z)=\frac{1}{e}+\frac{c_{\Psi}}{Z^{r-2}}\left(1+O\left(\frac{1}{Z^{r}}\right)\right)
\end{aligned}
$$

with non zero constants $c_{\sigma}, c_{P}$. Similar asymptotics hold for all higher order derivatives.

4. Non vanishing: there holds

$$
\forall Z \geq 0, \quad \rho_{P}>0
$$

5. Strict positivity inside the light cone: there exists $c=c(d, \ell, r)>0$ such that

$$
\forall 0 \leq Z \leq Z_{2}, \quad \begin{aligned}
& (1-w-\Lambda w)^{2}-(\sigma+\Lambda \sigma)^{2}>c \\
& 1-w-\Lambda w-\frac{(1-w)(\sigma+\Lambda \sigma)}{\sigma}>c
\end{aligned}
$$


6. Strict positivity outside the light cone:

$$
\exists c=c_{d, \ell, r}>0, \quad \forall Z \geq Z_{2}, \quad \begin{aligned}
& (1-w-\Lambda w)^{2}-(\sigma+\Lambda \sigma)^{2}>c, \\
& 1-w-\Lambda w>c .
\end{aligned}
$$

Remark 2.4 (Restriction on the parameters) The proof of Theorem 2.3 requires the non degeneracy of an explicit series $S_{\infty}(d, \ell) \neq 0$ which is numerically checked in [43] in the range (1.5). The positivity properties (2.21), (2.22) are checked analytically in [43] and will be fundamental for the well-posedness of the linearized flow inside the light cone, and the control of global Sobolev norms outside the light cone. Let us insist that the restriction on parameters relies on the intersection of the conditions (1.10), $S_{\infty}(d, \ell) \neq 0$ and (2.21), (2.22). The range (1.5) is just an example where this holds, but a larger range of parameters can be directly extracted from [43], and the conclusion of Theorem 1.1 would follow. In particular, since we are working with non vanishing solutions, the fact that the non linearity is an odd integer can be relaxed as in [44], hence providing an open range of parameters. Determining the exact range of validity of parameters for which Theorem 1.1 holds remains open.

Remark 2.5 The strict positivity property (2.21) inside the light cone will play a distinguished role in the analysis of the linearized of the operator and the derivation of the spectral gap which is the key to decay, see Proposition 3.10. Together with the strict positivity (2.22) outside the light cone, it will also allow us to derive energy bounds at high regularity, see Proposition 7.1.

From now on and for the rest of this paper, we assume (1.5). We observe from direct check that there holds:

$$
r^{*}(\ell)=\frac{d+\ell}{\ell+\sqrt{d}}>2 \Leftrightarrow \ell<d-2 \sqrt{d}=\ell_{2}(d) .
$$

Recalling (2.3), we may therefore assume from (2.18) that the blow speed $r=r_{k}$ satisfies

$$
r>2 \Leftrightarrow e=\frac{r-2}{r}>0 .
$$

\subsection{Linearization of the renormalized flow}

We look for $u$ solution to (1.1) and proceed to the decomposition of Lemma 2.1. We are left with finding a global, in self similar time $\tau \in\left[\tau_{0},+\infty\right)$, 
solution to (2.5):

$$
\mid \begin{gathered}
\partial_{\tau} \rho_{\mathrm{Tot}}=-\rho_{\mathrm{Tot}} \Delta \Psi_{\mathrm{Tot}}-\frac{\mu \ell(r-1)}{2} \rho_{\mathrm{Tot}}-\left(2 \partial_{Z} \Psi_{\mathrm{Tot}}+\mu Z\right) \partial_{Z} \rho_{\mathrm{Tot}} \\
\rho_{\mathrm{Tot}} \partial_{\tau} \Psi_{\mathrm{Tot}}=b^{2} \Delta \rho_{\mathrm{Tot}} \\
\quad-\left[\left|\nabla \Psi_{\mathrm{Tot}}\right|^{2}+\mu(r-2) \Psi_{\mathrm{Tot}}-1+\mu \Lambda \Psi_{\mathrm{Tot}}+\rho_{\mathrm{Tot}}^{p-1}\right] \rho_{\mathrm{Tot}}
\end{gathered}
$$

with non vanishing density $\rho_{\text {Tot }}>0$. We define

$$
\mid \begin{aligned}
& H_{2}=\mu+2 \frac{\Psi_{P}^{\prime}}{Z}=\mu(1-w), \\
& H_{1}=-\left(\Delta \Psi_{P}+\frac{\mu \ell(r-1)}{2}\right)=H_{2} \frac{\Lambda \rho_{P}}{\rho_{P}}=\frac{\mu \ell}{2}(1-w)\left[1+\frac{\Lambda \sigma}{\sigma}\right] .
\end{aligned}
$$

We linearize

$$
\rho_{\mathrm{Tot}}=\rho_{P}+\rho, \quad \Psi_{\mathrm{Tot}}=\Psi_{P}+\Psi
$$

and compute, using the profile Eq. (2.8), for the first equation:

$$
\begin{aligned}
\partial_{\tau} \rho= & -\left(\rho_{P}+\rho\right) \Delta\left(\Psi_{P}+\Psi\right)-\frac{\mu \ell(r-1)}{2}\left(\rho_{P}+\rho\right) \\
& -\left(2 \partial_{Z} \Psi_{P}+\mu Z+2 \partial_{Z} \Psi\right)\left(\partial_{Z} \rho_{P}+\partial_{Z} \rho\right) \\
= & -\rho_{\text {Tot }} \Delta \Psi-2 \nabla \rho_{\text {Tot }} \cdot \nabla \Psi+H_{1} \rho-H_{2} \Lambda \rho
\end{aligned}
$$

and for the second one:

$$
\begin{aligned}
\rho_{\mathrm{Tot}} \partial_{\tau} \Psi= & b^{2} \Delta \rho_{\mathrm{Tot}}-\rho_{\mathrm{Tot}}\left\{\left|\nabla \Psi_{P}\right|^{2}+2 \nabla \Psi_{P} \cdot \nabla \Psi+|\nabla \Psi|^{2}-1+\mu(r-2) \Psi_{P}\right. \\
& +\mu(r-2) \Psi+\mu\left(\Lambda \Psi_{P}+\Lambda \Psi\right) \\
& \left.+\left(\rho_{P}+\rho\right)^{p-1}\right\} \\
= & b^{2} \Delta \rho_{\mathrm{Tot}}-\rho_{\mathrm{Tot}}\left\{2 \nabla \Psi_{P} \cdot \nabla \Psi+\mu \Lambda \Psi+\mu(r-2) \Psi\right. \\
& \left.+|\nabla \Psi|^{2}+\left(\rho_{P}+\rho\right)^{p-1}-\rho_{P}^{p-1}\right\} \\
= & b^{2} \Delta \rho_{\mathrm{Tot}}-\rho_{\mathrm{Tot}}\left\{H_{2} \Lambda \Psi+\mu(r-2) \Psi\right. \\
& \left.+|\nabla \Psi|^{2}+(p-1) \rho_{P}^{p-2} \rho+\operatorname{NL}(\rho)\right\}
\end{aligned}
$$

with

$$
\mathrm{NL}(\rho)=\left(\rho_{P}+\rho\right)^{p-1}-\rho_{P}^{p-1}-(p-1) \rho_{P}^{p-2} \rho .
$$


We arrive at the exact (nonlinear) linearized flow

$$
\mid \begin{aligned}
& \partial_{\tau} \rho=H_{1} \rho-H_{2} \Lambda \rho-\rho_{\mathrm{Tot}} \Delta \Psi-2 \nabla \rho_{\mathrm{Tot}} \cdot \nabla \Psi, \\
& \partial_{\tau} \Psi=b^{2} \frac{\Delta \rho_{\mathrm{Tot}}}{\rho_{\mathrm{Tot}}}-\left\{H_{2} \Lambda \Psi+\mu(r-2) \Psi+|\nabla \Psi|^{2}\right. \\
& \left.\quad+(p-1) \rho_{P}^{p-2} \rho+\mathrm{NL}(\rho)\right\} .
\end{aligned}
$$

Theorem 1.1 is therefore equivalent to exhibiting a finite co-dimensional manifold of smooth well localized initial data leading to global, in renormalized $\tau$-time, solutions to (2.25).

\subsection{Strategy of the proof}

We now explain the strategy of the proof of Theorem 1.1.

Step 1 Wave equation and propagator estimate. After the change of variables $\Phi=\rho_{P} \Psi$, we may schematically rewrite the linearized flow (2.25) in the form

$$
\partial_{\tau} X=m X+\mathrm{NL}(X)-b^{2} \mid \begin{aligned}
& 0 \\
& \Delta\left(\rho_{P}+\rho\right)
\end{aligned}
$$

with

$$
X=\mid \begin{aligned}
& \rho \\
& \Phi
\end{aligned}, \quad m=\left(\begin{array}{ll}
H_{1}-H_{2} \Lambda & -\Delta+H_{3} \\
-(p-1) Q-H_{2} \Lambda & H_{1}-\mu(r-2)
\end{array}\right),
$$

where $Q, H_{1}, H_{2}, H_{3}$ are explicit potentials generated by the profile $\rho_{P}, \Psi_{P}$. During the first step the $b^{2} \Delta$ term is treated perturbatively. We commute the equation with the powers of the laplacian $\Delta^{k}$ and obtain for $X_{k}=\Delta^{k} X$

$$
\partial_{\tau} X_{k}=m_{k} X+\mathrm{NL}_{k}(X) .
$$

We then show that, provided $k$ is large enough, $m_{k}$ is a finite rank perturbation of a maximally dissipative operator with a spectral gap $\delta>0$. The topology in which maximal accretivity is established depends on the properties of the wave equation ${ }^{3}$ encoded in (2.28) and is based on weighted Sobolev norms with weights vanishing on the light cone corresponding to the point $P 2$ of the profile. Indeed, the principal part of the wave equation is roughly of the form

$$
\partial_{\tau}^{2} \rho-D(Z) \partial_{Z}^{2} \rho
$$

\footnotetext{
3 Reminiscent of the wave equation arising in a linearization of the compressible Euler equations.
} 
where the weight $D(Z)$ vanishes on the light cone $Z=Z_{2}$ corresponding to the $P_{2}$ point. The corresponding propagation estimates for the wave equation produce an priori control of the solution in the interior of the light cone $Z<Z_{2}$, modulo an a priori control of a finite number of directions corresponding to non positive eigenvalues of $m_{k}$. An essential structural fact of this step is the $e^{\infty}$ regularity of the profile. Indeed, we claim that for a generic non $e^{\infty}$ solution at $P 2$, the number of derivatives required to show accretivity of the linearized operator is always strictly greater than the regularity of the profile at $P_{2}$. As a result such profiles may be completely unstable and are not amenable to our analysis. The $e^{\infty}$ regularity obtained in [43] is therefore absolutely fundamental. The analytic properties leading to the maximality of the linearized operator will be consequences of (2.21), (2.22). We note that the coercivity constant in (2.21) degenerates as $r \rightarrow r^{*}$, and the number of derivatives needed for accretivity is inversely proportional to this constant. This is a manifestation of a quasilinear effect which is new for NLS: the problem sees a scaling which depends on the chosen self similar profile.

Step 2 Extension slightly beyond the light cone. Exponential decay estimates provided in the first step yield control in the interior of the light cone $Z<$ $Z_{2}$ only. It turns out that the analysis of the first step can be made more robust and extended ${ }^{4}$ slightly beyond the light cone, all the way to a spacelike hypersurface $Z=Z_{2}+a, 0<a \ll 1$, even though it is complicated by the dependence of the underlying wave equation on variable coefficients or, equivalently, on non constancy of the $Q(Z)$ term in (2.27). We can revisit the first step by producing a new maximal accretivity structure for a norm which does not generate in the zone $Z<Z_{2}+a, 0<a \ll 1$. The argument relies on a new generalized monotonicity formula. The corresponding propagation estimates recovers exponential decay in the extended zone $Z<Z_{2}+a$. Once decay has been obtained strictly beyond the light cone, a simple finite speed of propagation argument allows us to propagate decay to any compact set $Z<Z_{0}, Z_{0} \gg 1$.

Step 3 Loss of derivatives. The decay obtained in step 2 relies on energy estimates compatible with the wave propagation and the Eulerian structure of approximation. The full evolution however is that of the Schrödinger equation and contains the $b^{2} \Delta$ term on the right hand side of (2.26). Such a term leads to an unavoidable loss of one derivative. However, this loss comes with a $b^{2}$ smallness in front. We then argue as follows. We pick a large enough regularity level $k_{m}=k_{m}(r, d) \gg 2 k_{0}$, where $k_{0}$ is the power of the laplacian used for commutation in step 2, and derive a global Schrödinger like energy identity on the full flow (2.25). The choice of phase and modulus as basic variables turns the equation quasilinear and makes this identity rather complicated and

\footnotetext{
${ }^{4}$ Reminiscent of a non-characteristic energy estimate.
} 
unfamiliar. An essential difficulty, which is deeply related to step 2, is that at the highest level of derivatives, the non trivial space dependence of the profile measured by $Q(Z)=\rho_{P}^{p-1}(Z)$ in (2.27) produces a coupling term and a non trivial quadratic form. The condition (2.22) implies that the corresponding quadratic form is definite positive for $k_{m}$ large enough.

Step 4 Closing estimates. As explained above, we work with a linearized nonlinear equation, i.e., obtained after subtracting off the profile, written in terms of the phase and modulus unknowns $(\Psi, \rho)$, in renormalized self-similar variables $(\tau, Z)$, where the singularity corresponds to $(\tau=\infty, Z=0)$, a special light cone is $\left(\tau, Z=Z_{2}\right)$ and where in the original variables $(t, r)$ the region $r \geq 1$ corresponds to $Z \geq e^{\mu \tau}$.

First, outside the singularity $r \geq 1$, we modify the profile by strengthening its decay to make it rapidly decaying and of finite energy. Relative to the self-similar variables this modification happens at $Z \sim e^{\mu \tau}$, far from the singularity, and as a result is harmless. Then, we run two sets of estimates. First, we employ wave propagation like estimates which go initially just slightly beyond the special light cone and then extend to any compact set in $Z$. These estimates are carried out at a sufficiently high level of regularity with $\sim 2 k_{0}$ derivatives. The number $k_{0}$ emerges from the linear theory and is determined by the (conditional) positivity of a certain quadratic form responsible for maximal accretivity.

Then, we couple these estimates to global Schrödinger like estimates which take into account previously ignored $b^{2} \Delta$ and take care of global control. These estimates are carried out at all levels of regularity up to $k_{m}$ derivatives with $k_{m} \gg k_{0}$. They are carefully designed weighted $L^{2}$ type estimates. The weights depend on the number of derivatives $k$ : at first, their strength grows with $k$ but by the time we reach the highest level of regularity $k_{m}$ the weight function is identically $=1$. The latter has to do with a well-known fact that even for a linear Schrödinger equation, use of weights leads to a derivative loss ( $\Delta$ is not self-adjoint on a weighted $L^{2}$ space.) Therefore, our highest derivative norm should correspond to an unweighted $L^{2}$ estimate. Of course, this last estimate also sees a positivity condition (2.22) responsible for the coercivity of an appearing quadratic form.

These global weighted $L^{2}$ bounds then allow us to prove pointwise bounds for the solution and its derivatives which, in turn, allow us to control nonlinear terms. The obtained sets of weighted $L^{\infty}$ bounds on derivatives recover in particular the non vanishing assumption required of the solution. We should note that while all the local (in $Z$ ) norms decay exponentially in $\tau$, the global norms are merely bounded. In the original $(t, r)$ variables this means that the perturbation decays inside and slightly beyond the backward light cone from the singular point but does not decay away from the singularity. This is, of course, entirely consistent with the global conservation of energy for NLS. 
The whole proof proceeds via a bootstrap argument which also involves a Brouwer type argument to deal with unstable modes, if any, arising in linear theory of step 1 . This is what produces a finite co-dimension manifold of admissible data.

\section{Linear theory slightly beyond the light cone}

Our aim in this section is to study the linearized problem (2.25) for the exact Euler problem $b=0$. We in particular aim at setting up the suitable functional framework in order to apply classical propagator estimates which will yield exponential decay on compact sets $Z \lesssim 1$ modulo the control of a finite number of unstable directions.

\subsection{Growth bounds for dissipative operators}

We start this section by recalling classical facts about unbounded operators and their semigroups. Let $(H,\langle\cdot, \cdot\rangle)$ be a hermitian Hilbert space and $A$ be a closed operator with a dense domain $D(A)$. We recall the definition of the adjoint operator $A^{*}$ : let

$$
\begin{aligned}
D\left(A^{*}\right)=\{X \in H, \tilde{X} \in D(A) \mapsto & \langle X, A \tilde{X}\rangle \\
& \text { extends as a bounded functional on } H\},
\end{aligned}
$$

then $A^{*} X$ is given by the Riesz theorem as the unique element of $H$ such that

$$
\forall \tilde{X} \in D(A),\left\langle A^{*} X, \tilde{X}\right\rangle=\langle X, A \tilde{X}\rangle .
$$

Let $\sigma(A)$ denote the spectrum of $A$, i.e., the complement of the resolvent set. We recall the following classical lemma.

Lemma 3.1 (Properties of maximal dissipative operators, [56] p. 49) Let A be a maximal dissipative operator on a Hilbert space $H$ with domain $D(A)$, then:

(i) A is closed;

(ii) $A^{*}$ is maximal dissipative;

(iii) $\sigma(A) \subset\{\lambda \in \mathbb{C}, \Re(\lambda) \leq 0\}$;

(iv) $\left\|(A-\lambda)^{-1}\right\| \leq|\Re(\lambda)|^{-1}$ for $\Re(\lambda)>0$.

We now recall from Hille-Yoshida's theorem that a maximally dissipative operator $A_{0}$ generates a strongly continuous semigroup $T_{0}$ on $H$, and so does $A_{0}+K$ for any bounded perturbation $K$. Let us now recall the following classical properties of strongly continuous semigroup $T(t)$. 
Proposition 3.2 (Growth bound, [20] Cor 2.11, p. 258) Let the growth bound of the semigroup be defined as

$$
w_{0}=\inf \left\{w \in \mathbb{R}, \exists M_{w} \text { such that } \forall t \geq 0,\|T(t)\| \leq M_{w} e^{w t}\right\} .
$$

Let $w_{\mathrm{ess}}$ denote the essential growth bound of the semigroup:

$$
w_{\mathrm{ess}}=\inf \left\{w \in \mathbb{R}, \exists M_{w} \text { such that } \forall t \geq 0,\|T(t)\|_{\mathrm{ess}} \leq M_{w} e^{w t}\right\}
$$

with

$$
\|T(t)\|_{\mathrm{ess}}=\inf _{K \in \mathscr{K}(H)}\|T(t)-K\|_{H \rightarrow H}
$$

and $\mathscr{K}(H)$ is the ideal of compact operators on $H$; and let

$$
s(A)=\sup \{\Re(\lambda), \quad \lambda \in \sigma(A)\} .
$$

Then

$$
w_{0}=\max \left\{w_{\mathrm{ess}}, s(A)\right\}
$$

and

$$
\forall w>w_{\mathrm{ess}} \text {, the set } \Lambda_{w}(A):=\sigma(A) \cap\{\Re(\lambda)>w\} \text { is finite. }
$$

Moreover, each eigenvalue $\lambda \in \Lambda_{w}(A)$ has finite algebraic multiplicity $m_{\lambda}^{a}$ : $\exists k_{\lambda} \in \mathbb{Z}$ such that

$$
\begin{aligned}
& \operatorname{ker}(A-\lambda I)^{k_{\lambda}} \neq \emptyset, \quad \forall k \geq k_{\lambda}, \operatorname{ker}(A-\lambda I)^{k}=\operatorname{ker}(A-\lambda I)^{k_{\lambda}}, \\
& m_{\lambda}^{a}:=\operatorname{dim} \operatorname{ker}(A-\lambda I)^{k_{\lambda}}
\end{aligned}
$$

We note that the subspaces $V_{w}(A)=\cup_{\lambda \in \Lambda_{w}(A)} \operatorname{ker}(A-\lambda I)^{k_{\lambda}}$ and $V_{w}^{\perp}\left(A^{*}\right)$ are invariant for $A$. In particular, $A\left(D(A) \cap V_{w}^{\perp}\left(A^{*}\right)\right) \subset V_{w}^{\perp}\left(A^{*}\right)$. The invariance $V_{w}(A)$ is immediate. To show that $A\left(D(A) \cap V_{w}^{\perp}\left(A^{*}\right)\right) \subset V_{w}^{\perp}\left(A^{*}\right)$ we let $X \in D(A) \cap V_{w}^{\perp}\left(A^{*}\right), Y \in V_{w}\left(A^{*}\right)$ and consider $\langle A X, Y\rangle$. Since $Y \in D\left(A^{*}\right)$ and $V_{w}\left(A^{*}\right)$ is invariant for $A^{*}$,

$$
\langle A X, Y\rangle=\left\langle X, A^{*} Y\right\rangle=0 .
$$

We claim the following corollary. 
Lemma 3.3 (Perturbative exponential decay) Let $T_{0}$ be the strongly continuous semigroup generated by a maximal dissipative operator $A_{0}$, and $T$ be the strongly continuous semi group generated by $A=A_{0}+K$ where $K$ is a compact operator on $H$. Then for any $\delta>0$, the following holds:

(i) the set $\Lambda_{\delta}(A)=\sigma(A) \cap\{\lambda \in \mathbb{C}, \Re(\lambda)>\delta\}$ is finite, each eigenvalue $\lambda \in \Lambda_{\delta}(A)$ has finite algebraic multiplicity $k_{\lambda}$. In particular, the subspace $V_{\delta}(A)$ is finite dimensional;

(ii) We have $\Lambda_{\delta}(A)=\overline{\Lambda_{\delta}\left(A^{*}\right)}$ and $\operatorname{dim} V_{\delta}\left(A^{*}\right)=\operatorname{dim} V_{\delta}(A)$. The direct sum decomposition

$$
H=V_{\delta}(A) \bigoplus V_{\delta}^{\perp}\left(A^{*}\right)
$$

is preserved by $T(t)$ and there holds:

$$
\forall X \in V_{\delta}^{\perp}\left(A^{*}\right), \quad\|T(t) X\| \leq M_{\delta} e^{\delta t}\|X\| .
$$

(iii) The restriction of $A$ to $V_{\delta}(A)$ is given by a direct sum of $\left(m_{\lambda} \times m_{\lambda}\right)_{\lambda \in \Lambda_{\delta}(A)}$ matrices each of which is the Jordan block associated to the eigenvalue $\lambda$ and the number of Jordan blocks corresponding to $\lambda$ is equal to the geometric multiplicity of $\lambda-m_{\lambda}^{g}=\operatorname{dimker}(A-\lambda I)$. In particular, $m_{\lambda}^{a} \leq m_{\lambda}^{g} k_{\lambda}$. Each block corresponds to an invariant subspace $J_{\lambda}$ and the semigroup $T$ restricted to $J_{\lambda}$ is given by the matrix

$$
\left.T(t)\right|_{J_{\lambda}}=\left(\begin{array}{cccc}
e^{\lambda t} & t e^{\lambda t} & \cdots & t^{m_{\lambda}-1} e^{\lambda t} \\
0 & e^{\lambda t} & \cdots & t^{m_{\lambda}-2} e^{\lambda t} \\
\cdots & & & \\
0 & 0 & \cdots & e^{\lambda t}
\end{array}\right)
$$

Proof This is a simple consequence of Proposition 3.2.

Step 1 Perturbative bound. First, since $A_{0}$ is maximally dissipative,

$$
\forall t \geq 0, \quad\left\|T_{0}(t)\right\| \lesssim 1
$$

implies $w_{0}\left(A_{0}\right) \leq 0$. By Proposition 3.2, $s\left(A_{0}\right) \leq 0$ and

$$
w_{\text {ess }}\left(T_{0}\right) \leq 0
$$

On the other hand, from [20] Prop 2.12 p. 258, compactness of $K$ implies

$$
w_{\mathrm{ess}}(T)=w_{\mathrm{ess}}\left(T_{0}\right) \leq 0
$$


Let now $\lambda \in \sigma(A)$ with $\Re(\lambda)>0$, then the formula

$$
A-\lambda=A_{0}+K-\lambda=\left(A_{0}-\lambda\right)\left(\operatorname{Id}+\left(A_{0}-\lambda\right)^{-1} K\right)
$$

and invertibility of $\left(A_{0}-\lambda\right)$ imply that $\lambda$ belongs to the spectrum of the Fredholm operator Id $+\left(A_{0}-\lambda\right)^{-1} K$. Therefore, $\lambda$ is an eigenvalue of $A$. On the other hand, $\Re(\lambda)>\delta$ implies $\Re(\lambda)>\delta>0 \geq w_{\text {ess }}(T)$, and hence, by (3.2), there are finitely many eigenvalues with $\Re(\lambda)>\delta$. In fact, Proposition 3.2 also directly shows that each some $\lambda$ is an eigenvalue and implies the rest of (i).

Since $A^{*}=A_{0}^{*}+K^{*}$ and $A_{0}^{*}$ is maximally dissipative from Lemma 3.1, we can run the same argument as above for $A^{*}$. Moreover, $\sigma(A)=\overline{\sigma\left(A^{*}\right)}$ ([56], Prop. 2.7), (i) is proved.

The argument above, in fact, shows that $\{\lambda \in \mathbb{C}, \mathfrak{R}(\lambda)>\delta\} \cap\{\lambda \in \sigma(A)\}$ is finite, since for every $\Re(\lambda)>0$ and $\lambda \in \sigma(A), \lambda$ is an eigenvalue of $A$.

Step 2 The first statement of (ii) is standard. We already explained that the subspaces $V_{\delta}(A)$ and $D(A) \cap V_{\delta}^{\perp}\left(A^{*}\right)$ are invariant for $A$. To prove the direct decomposition we recall that the subspace $V_{\delta}(A)$ is the image of $H$ under the spectral projection $P_{\delta}(A)$ associated to the set $\Lambda_{\delta}(A)$ :

$$
P_{\delta}(A)=\frac{1}{2 \pi i} \int_{\Gamma} \frac{d \lambda}{\lambda I-A}
$$

where $\Gamma$ is an arbitrary contour containing the set $\Lambda_{\delta}(A)$. There is a direct decomposition

$$
H=\operatorname{Im} P_{\delta}(A) \bigoplus k \operatorname{er} P_{\delta}(A)
$$

On the other hand, the adjoint

$$
P_{\delta}^{*}(A)=\frac{1}{2 \pi i} \int_{\bar{\Gamma}} \frac{d \lambda}{\lambda I-A^{*}}=P_{\delta}\left(A^{*}\right)
$$

is the spectral projection of $A^{*}$ associated to the set $\overline{\Lambda_{\delta}(A)}$. The result is now immediate.

Step 3 Semigroups generated by restriction and conclusion. Let $V=V_{\delta}(A)$, $U=V_{\delta}^{\perp}\left(A^{*}\right)$ and $P$ denote the projection on $V_{\delta}^{\perp}\left(A^{*}\right)$ in the direct decomposition (3.3). Let $\tilde{A}$ denote the restriction of $A$ to $U$ with the domain $D(\tilde{A})=U \cap D(A)$. By invariance

$$
\forall X \in U \cap D(A), \quad \tilde{A} X=A X .
$$


Let $\tilde{T}$ be the semigroup on $U$ generated by $\tilde{A}=A$. Then for all $X \in$ $D(A) \cap U, \tilde{T}(t) X \in C^{1}([0,+\infty), D(\tilde{A}))$ is the unique strong solution to the ode

$$
\frac{d X(t)}{d t}=A X(t), \quad X(0)=X .
$$

This implies that $\tilde{T}(t) X=T(t) X$ for all $X \in D(A) \cap U$ and thus for all $X \in U$ by continuity of the semigroup. By Proposition 3.2 the growth bound of $\tilde{T}$ satisfies

$$
w_{0}(\tilde{T}) \leq \max \left\{w_{\mathrm{ess}}(\tilde{T}), s(\tilde{A})\right\}
$$

We first argue that

$$
w_{\mathrm{ess}}(\tilde{T}) \leq 0
$$

To prove that we note that we already established that $w_{\text {ess }}(T) \leq 0$. We then fix $\varepsilon>0$ and, for any $t \geq 0$ choose a compact operator $K(t) \in \mathcal{K}(H)$ on $H$ such that

$$
\log \|T(t)-K(t)\|_{H \rightarrow H}<\varepsilon t+\log M
$$

for some constant $M$ which may depend on $\varepsilon$. The restriction $\tilde{K}(t)=P K(t)$ of $K(t)$ to $U$ is a compact operator on $U$. Then, for any $t \geq 0$

$$
\begin{aligned}
\log \|\tilde{T}(t)-\tilde{K}(t)\|_{U \rightarrow U} & =\log \|P(T(t)-K(t))\|_{U \rightarrow U} \\
& \leq \log \left\{C_{P}\|T(t)-K(t)\|_{H \rightarrow H}\right\} \\
& <\log C_{P}+\log M+\varepsilon t,
\end{aligned}
$$

where $C_{P}$ denotes the norm of the projector $P$. The desired conclusion follows.

To show that $s(\tilde{A}) \leq \delta$ we assume that $\lambda \in \sigma(\tilde{A})$ with $\Re(\lambda)>\delta$, then $\lambda$ is an eigenvalue of $\tilde{A}$ and, by invariance of $U, \lambda$ is an eigenvalue of $A$ with a non-trivial eigenvector $\psi \in U$. However, by construction, all such $\psi$ belong to the subspace $V=V_{\delta}(A)$, contradiction. Hence $s(\tilde{A}) \leq \delta$ and Proposition 3.2 yields (3.4).

Finally, part (iii) is completely standard.

We will use Lemma 3.3 in the following form.

Lemma 3.4 (Exponential decay modulo finitely many instabilities) Let $\delta>0$ and let $T_{0}$ be the strongly continuous semigroup generated by a maximal dissipative operator $A_{0}$, and $T$ be the strongly continuous semigroup generated 
by $A=A_{0}-\delta+K$ where $K$ is a compact operator on $H$. Let the (possibly empty) finite set

$$
\Lambda=\{\lambda \in \mathbb{C}, \Re(\lambda) \geq 0\} \cap\{\lambda \text { is an eigenvalue of } A\}=\left(\lambda_{i}\right)_{1 \leq i \leq N}
$$

and let

$$
H=U \bigoplus V,
$$

where $U$ and $V$ are invariant subspaces for $A$ and $V$ is the image of the spectral projection of $A$ associated to the set $\Lambda$. Then there exist $C, \delta_{g}>0$ such that

$$
\forall X \in U, \quad\|T(t) X\| \leq C e^{-\frac{\delta g}{2} t}\|X\| .
$$

Proof We apply Lemma 3.3 to $\tilde{A}=A+\delta=A_{0}+K$ with generates the semi group $\tilde{T}$. Hence the set

$$
\Lambda_{\frac{\delta}{4}}(\tilde{A})=\left\{\lambda \in \mathbb{C}, \Re(\lambda)>\frac{\delta}{4}\right\} \cap\{\lambda \text { is an eigenvalue of } \tilde{A}\}
$$

is finite. Moreover

$$
A X=\lambda X \Leftrightarrow \tilde{A} X=(\lambda+\delta) X
$$

and hence

$$
\Lambda \subset \Lambda_{\frac{\delta}{4}}
$$

Let

$$
H=U_{\delta} \bigoplus V_{\delta}
$$

be the invariant decomposition of $\tilde{A}$ (and of $A$ ) associated to the set $\Lambda_{\frac{\delta}{4}}$. Clearly, $U_{\delta} \subset U$ and

$$
U=U_{\delta} \bigoplus O_{\delta}
$$

where $O_{\delta}$ is the image of the spectral projection of $A$ associated with the set $\Lambda_{\frac{\delta}{4}} \backslash \Lambda$. By Lemma 3.3,

$$
\forall X \in U_{\delta}, \quad\|\tilde{T}(t) X\| \leq M_{\delta} e^{\frac{\delta}{4} t}\|X\|,
$$


which implies

$$
\forall X \in U_{\delta}, \quad\|T(t) X\|=e^{-\delta t}\|\tilde{T}(t) X\| \leq M_{\delta} e^{-\frac{3 \delta}{4} t}\|X\| .
$$

Let now $X \in U$. Since $U_{\delta}$ is invariant by $T$ and (3.6) yields exponential decay on $U_{\delta}$, we assume $X \in O_{\delta}$. $O_{\delta}$ is an invariant subspace of $A$ generated by the eigenvalues $\lambda$ with the property that $-\frac{3}{4} \delta \leq \Re(\lambda)<0$. Let $\delta_{g}>0$ be defined as

$$
-\delta_{g}:=\sup \left\{\Re(\lambda):-\frac{3}{4} \delta \leq \Re(\lambda)<0\right\}
$$

From part (iii) of Lemma 3.3,

$$
\|T(t) X\|_{O_{\delta}} \leq C \sup _{\Re(\lambda)<0} e^{\lambda t} t^{m_{\lambda}-1}\|X\| \leq C^{-\frac{\delta g}{2} t}\|X\| .
$$

This concludes the proof of Lemma 3.4.

Our final result in this section is to set up a Brouwer type argument for the evolution of unstable modes.

Lemma 3.5 Let $A, \delta_{g}$ as in Lemma 3.4 with the decomposition

$$
H=U \bigoplus V
$$

into stable and unstable subspaces Fix a sufficiently large $t_{0}>0$ (dependent on A). Let $F(t, x)$ such that, $\forall t \geq t_{0}, F(t, x) \in V$, depends continuously on $x$ and

$$
\|F(t, x)\| \leq e^{-\frac{2 \delta g}{3} t}
$$

be given. Let $x(t)$ denote the solution to the ode

$$
\mid \begin{aligned}
& \frac{d x}{d t}=A x+F(t, x) \\
& x\left(t_{0}\right)=x_{0} \in V
\end{aligned}
$$

Then, for any $x_{0}$ in the ball

$$
\left\|x_{0}\right\| \leq e^{-\frac{3 \delta g}{5} t_{0}}
$$

we have

$$
\|x(t)\| \leq e^{-\frac{\delta g}{2} t}, \quad t_{0} \leq t \leq t_{0}+\Gamma
$$


for some large constant $\Gamma$ (which only depends on $A$ and $t_{0}$.) Moreover, there exists $x^{*} \in V$ in the same ball as a above such that $\forall t \geq t_{0}$ the solution $x(t)$ with initial data $x\left(t_{0}\right)=x^{*}$ obeys

$$
\|x(t)\| \leq e^{-\frac{3 \delta g}{5} t} .
$$

Proof According to Lemma 3.3 the subspace $V$ can be further decomposed into invariant subspaces on which $A$ is represented by Jordan blocks. We may therefore assume that $V$ is irreducible and corresponds to a Jordan block of $A$ of length $m_{\lambda}$ associated with an eigenvalue $\lambda$ with $\Re(\lambda) \geq 0$ and restrict $A$ to $V$. We decompose $A$ as

$$
A=\lambda I+N,
$$

where $N$ has the property that $N^{m_{\lambda}-1}=0$, and

$$
e^{t N}=\left(\begin{array}{cccc}
1 & t & \cdots & t^{m_{\lambda}-1} \\
0 & 1 & \cdots & t^{m_{\lambda}-2} \\
\cdots & & \\
0 & 0 & \cdots & 1
\end{array}\right) .
$$

The claim (3.7) follows from the growth on the Jordan block:

$$
\begin{aligned}
\|x(t)\| & =\left\|e^{\left(t-t_{0}\right) A} x_{0}+\int_{t_{0}}^{t} e^{(t-\tau) A} F(\tau, x) d \tau\right\| \\
& \leq C \Gamma^{m_{\lambda}-1} e^{\Re(\lambda) \Gamma} e^{-\frac{3 \delta_{g} t_{0}}{5}}+\int_{t_{0}}^{t} C\left|\tau-t_{0}\right|^{m_{\lambda}-1} e^{\Re(\lambda)(t-\tau)} e^{-\frac{2}{3} \delta_{g} \tau} d \tau \\
& \leq C \Gamma^{m_{\lambda}-1} e^{\Re(\lambda) \Gamma} e^{-\frac{3 \delta_{g} t_{0}}{5}}
\end{aligned}
$$

and hence the size of constant $\Gamma$ is determined from the inequality

$$
C \Gamma^{m_{\lambda}-1} e^{\Re(\lambda) \Gamma} e^{-\frac{3 \delta_{g} t_{0}}{5}} \leq e^{-\frac{\delta}{2}\left(t_{0}+\Gamma\right)},
$$

a sufficient condition being

$$
\Gamma \leq \frac{t_{0}}{2}\left[\frac{\delta_{g}}{10 \Re(\lambda)+5 \delta_{g}}\right],
$$

which can be made arbitrarily large by a choice of $t_{0}$.

We now define a new variable

$$
Y(t)=e^{-t N} e^{\frac{19 \delta_{g}}{30} t} x(t)
$$


Since $N$ and $A$ commute,

$$
\frac{d Y}{d t}=\left(\lambda+\frac{19 \delta_{g}}{30}\right) Y+\tilde{F}(t, Y), \quad Y\left(t_{0}\right)=y
$$

where $\tilde{F}(t, Y)=e^{-t N} e^{\frac{19 \delta g}{30} t} F(t, x)$ and

$$
\|\tilde{F}(t, Y)\| \lesssim e^{-\frac{\delta g}{31} t} .
$$

Since $t_{0}$ was chosen to be sufficiently large, we can assume that $\forall t \geq t_{0}$

$$
\|\tilde{F}(t, Y)\| \lesssim \epsilon e^{-\frac{\delta g}{60} t}
$$

and $\epsilon<\Re(\lambda)+\frac{19 \delta_{g}}{60}$. We now run a standard Brouwer type argument for $Y$. For any $y$ such that $\|y\| \leq 1$ we define the exit time $t^{*}$ to be the first time such that $\left\|Y\left(t^{*}\right)\right\|=1$. If for some $y, t^{*}=\infty$, we are done. Otherwise, assume that for all $\|y\| \leq 1, t^{*}<\infty$ and define the map $\Phi: B \rightarrow S$ as $\Phi(y)=Y\left(t^{*}\right)$ mapping the unit ball to the unit sphere. Note that $\Phi$ is the identity map on the boundary of $B$. To prove continuity of $\Phi$ we compute

$$
\frac{d\|Y\|^{2}}{d t}\left(t^{*}\right)=2 \Re(\lambda)+\frac{19 \delta_{g}}{15}+2 \Re\left\langle\tilde{F}\left(t^{*}, Y\left(t^{*}\right)\right), Y\left(t^{*}\right)\right\rangle \geq \frac{19 \delta_{g}}{30}>0 .
$$

This is the outgoing condition which implies continuity. The Brouwer argument applies and shows that such $\Phi$ can not exist. We now reinterpret the result in terms of $x$. We have shown existence of the data $x^{*}$ such that the corresponding solution $x(t)$ has the property that $\forall t \geq t_{0}$,

$$
\left\|e^{-t N} x(t)\right\| \leq e^{-\frac{19 \delta_{g}}{30} t} .
$$

Now $e^{-t N}$ is an invertible operator with the inverse given by $e^{t N}$ and its norm bounded by $C t^{m_{\lambda}-1}$. The result follows immediately. We note that the resulting solution $x(t)$ has initial data $x\left(t_{0}\right)$ in the ball $\left\|x\left(t_{0}\right)\right\| \leq e^{-\frac{3 \delta g}{5} t_{0}}$.

\subsection{Linearized equations}

Recall the exact linearized flow (2.25) which we rewrite:

$$
\mid \begin{aligned}
\partial_{\tau} \rho= & H_{1} \rho-H_{2} \Lambda \rho-\rho_{P} \Delta \Psi-2 \nabla \rho_{P} \cdot \nabla \Psi-\rho \Delta \Psi-2 \nabla \rho \cdot \nabla \Psi \\
\partial_{\tau} \Psi= & b^{2} \frac{\Delta \rho_{\mathrm{Tot}}}{\rho_{\mathrm{Tot}}}-\left\{H_{2} \Lambda \Psi+\mu(r-2) \Psi+(p-1) \rho_{P}^{p-2} \rho\right. \\
& \left.+|\nabla \Psi|^{2}+\mathrm{NL}(\rho)\right\} .
\end{aligned}
$$


Our aim for the remainder of the section is to find a Hilbert space in which the linearized operator is accretive modulo a compact perturbation in order to apply the general results of the previous section.

We introduce the new unknown

$$
\Phi=\rho_{P} \Psi
$$

and obtain equivalently using (2.24):

$$
\mid \begin{aligned}
& \partial_{\tau} \rho=H_{1} \rho-H_{2} \Lambda \rho-\Delta \Phi+H_{3} \Phi+G_{\rho}, \\
& \partial_{\tau} \Phi=-(p-1) Q \rho-H_{2} \Lambda \Phi+\left(H_{1}-\mu(r-2)\right) \Phi+G_{\Phi}
\end{aligned}
$$

with

$$
Q=\rho_{P}^{p-1}, \quad H_{3}=\frac{\Delta \rho_{P}}{\rho_{P}}
$$

and the nonlinear terms:

$$
\mid \begin{aligned}
& G_{\rho}=-\rho \Delta \Psi-2 \nabla \rho \cdot \nabla \Psi, \\
& G_{\Phi}=-\rho_{P}\left(|\nabla \Psi|^{2}+\mathrm{NL}(\rho)\right)+\frac{b^{2} \rho_{P}}{\rho_{\text {Tot }}} \Delta \rho_{\text {Tot }} .
\end{aligned}
$$

We transform (3.9) into a wave equation for $\Phi$ and compute:

$$
\begin{aligned}
\partial_{\tau}^{2} \Phi= & -(p-1) Q\left(H_{1} \rho-H_{2} \Lambda \rho-\Delta \Phi+H_{3} \Phi+G_{\rho}\right)+\partial_{\tau} G_{\Phi} \\
& -H_{2} \Lambda \partial_{\tau} \Phi+\left(H_{1}-\mu(r-2)\right) \partial_{\tau} \Phi \\
= & -(p-1) Q\left(-\Delta \Phi+H_{3} \Phi\right)-H_{2} \Lambda \partial_{\tau} \Phi+\left(H_{1}-\mu(r-2)\right) \partial_{\tau} \Phi \\
& +(p-1) Q\left(-H_{1}+H_{2} \Lambda\right)\left\{\frac { 1 } { ( p - 1 ) Q } \left[-\partial_{\tau} \Phi-H_{2} \Lambda \Phi\right.\right. \\
& \left.\left.+\left(H_{1}-\mu(r-2)\right) \Phi+G_{\Phi}\right]\right\}+\partial_{\tau} G_{\Phi}-(p-1) Q G_{\rho} \\
= & (p-1) Q \Delta \Phi-H_{2}^{2} \Lambda^{2} \Phi-2 H_{2} \Lambda \partial_{\tau} \Phi+A_{1} \Lambda \Phi+A_{2} \partial_{\tau} \Phi+A_{3} \Phi \\
& +\partial_{\tau} G_{\Phi}-\left(H_{1}+H_{2} \frac{\Lambda Q}{Q}\right) G_{\Phi}+H_{2} \Lambda G_{\Phi}-(p-1) Q G_{\rho}
\end{aligned}
$$

with

$$
\mid \begin{aligned}
& A_{1}=H_{2} H_{1}-H_{2} \Lambda H_{2}+H_{2}\left(H_{1}-\mu(r-2)\right)+H_{2}^{2} \frac{\Lambda Q}{Q}, \\
& A_{2}=2 H_{1}-\mu(r-2)+H_{2} \frac{\Lambda Q}{Q}, \\
& A_{3}=-\left(H_{1}-e\right) H_{1}+H_{2} \Lambda H_{1}-H_{2}\left(H_{1}-\mu(r-2)\right) \frac{\Lambda Q}{Q}-(p-1) Q H_{3} .
\end{aligned}
$$

In this section we focus on deriving decay estimates for (3.9). 
Remark 3.6 (Null coordinates and red shift) We note that the principal symbol of the above wave equation is given by the second order operator

$$
\square_{Q}:=\partial_{\tau}^{2}-\left((p-1) Q-H_{2}^{2} Z^{2}\right) \partial_{Z}^{2}+2 H_{2} Z \partial_{Z} \partial_{\tau} .
$$

In the variables of Emden transform this can be written equivalently as

$$
\square_{Q}=\partial_{\tau}^{2}-\mu^{2}\left[\sigma^{2}-(1-w)^{2}\right] \partial_{\varkappa}^{2}+2 \mu(1-w) \partial_{\varkappa} \partial_{\tau} .
$$

The two principal null direction associated with the above equation are

$$
L=\partial_{\tau}+\mu[(1-w)-\sigma] \partial_{\varkappa}, \quad \underline{L}=\partial_{\tau}+\mu[(1-w)+\sigma] \partial_{\varkappa}
$$

so that

$$
\square_{Q}=L \underline{L} .
$$

We observe that at $P 2$, we have $L=\partial_{\tau}$ and the surface $Z=Z_{2}$ is a null line (cone, if we view from the point of view of the higher dimensional space where a point $(\tau, Z)$ is in fact a $(d-1)$-dimensional sphere). Moreover, the associated acoustical metric is

$$
g_{Q}=\mu^{2} \Delta d \tau^{2}-2 \mu(1-w) d \tau d \varkappa+d \varkappa^{2}, \quad \Delta=(1-w)^{2}-\sigma^{2}
$$

for which $\partial_{\tau}$ is a Killing field (generator of translation symmetry). Therefore, $Z=Z_{2}$ is a Killing horizon (generated by a null Killing field.) We can make it even more precise by transforming the metric $g_{Q}$ into a slightly different form by defining the coordinate $s$ :

$$
s=\mu \tau-f(\varkappa), \quad f^{\prime}=\frac{1-w}{\Delta}
$$

so that

$$
g_{Q}=\Delta(d s)^{2}-\frac{\sigma^{2}}{\Delta} d \varkappa^{2}
$$

and then the coordinate $x^{*}$ :

$$
x^{*}=\int \frac{\sigma}{\Delta} d x
$$

so that

$$
g_{Q}=\Delta d\left(s+x^{*}\right) d\left(s-x^{*}\right)
$$


and $s+x^{*}$ and $s-x^{*}$ are the null coordinates of $g_{Q}$. The Killing horizon $Z=Z_{2}$ corresponds to $x^{*}=-\infty$ and $\Delta \sim e^{C x^{*}}$ for some positive constant $C$. In this form, near $Z_{2}$ the metric $g_{Q}$ resembles the $1+1$-quotient Schwarzschild metric near the black hole horizon. Note that the region $Z>Z_{2}$ corresponds to the interior of a black hole in a sense that the null geodesics of the acoustical metric never leave that region.

The associated surface gravity $\kappa$ which can be computed according to

$$
\begin{aligned}
\kappa & =\left.\frac{\partial_{x^{*} \Delta}}{2 \Delta}\right|_{P 2}=\left.\frac{\partial_{x} \Delta}{2 \sigma}\right|_{P 2}=\left.\frac{-w^{\prime}(1-w)-\sigma^{\prime} \sigma}{\sigma}\right|_{P 2} \\
& =\left.\left(-w^{\prime}-\sigma^{\prime}\right)\right|_{P 2}=1-w-\Lambda w-\left.\frac{(1-w) F}{\sigma}\right|_{P 2}>0 .
\end{aligned}
$$

This is precisely the positivity condition (2.21) (at P2). The positivity of surface gravity implies the presence of the red shift effect along $Z=Z_{2}$ both as an optical phenomenon for the acoustical metric $g_{Q}$ and also as an indicator of local monotonicity estimates for solutions of the wave equation $\square_{Q} \varphi=$ 0 , [17]. Near $Z_{2}$, the null characteristics spread out and the monotonicity estimates can be captured with the energy estimates based on a multiplier transversal to the set $Z=Z_{2}$, while the standard energy estimates based on the multiplier $\partial_{\tau}$ would be degenerate. The complication in the analysis below is the presence of lower order terms in the wave equation as well as the need for global in space estimates.

\subsection{The linearized operator}

The degeneracy of wave operator $\square_{Q}$ is a feature of the chosen coordinate system and, specifically, of the fact that $\partial_{\tau}$ is tangent to the set $Z=Z_{2}$. We can remedy this by adding to $\partial_{\tau}$ a small amount of $\Lambda$-derivative near $Z_{2}$. The precise technical implementation is as follows.

Pick a small enough parameter

$$
0<a \ll 1
$$

and consider the new variable

$$
T=\partial_{\tau} \Phi+a H_{2} \Lambda \Phi
$$

then

$$
\begin{aligned}
\partial_{\tau} T & =\partial_{\tau}^{2} \Phi+a H_{2} \Lambda \partial_{\tau} \Phi=\partial_{\tau}^{2} \Phi+a H_{2} \Lambda\left(T-a H_{2} \Lambda \Phi\right) \\
& =\partial_{\tau}^{2} \Phi+a H_{2} \Lambda T-a^{2} H_{2} \Lambda H_{2} \Lambda \Phi-a^{2} H_{2}^{2} \Lambda^{2} \Phi,
\end{aligned}
$$


which yields the $(T, \Phi)$ equation

$$
\partial_{\tau} \Phi=T-a H_{2} \Lambda \Phi
$$

and

$$
\begin{aligned}
\partial_{\tau} T= & (p-1) Q \Delta \Phi-H_{2}^{2} \Lambda^{2} \Phi-2 H_{2} \Lambda\left(T-a H_{2} \Lambda \Phi\right) \\
& +A_{1} \Lambda \Phi+A_{2}\left(T-a H_{2} \Lambda \Phi\right)+A_{3} \Phi \\
& +a H_{2} \Lambda T-a^{2} H_{2} \Lambda H_{2} \Lambda \Phi-a^{2} H_{2}^{2} \Lambda^{2} \Phi+G_{T} \\
= & (p-1) Q \Delta \Phi-(1-a)^{2} H_{2}^{2} \Lambda^{2} \Phi \\
& +\tilde{A}_{2} \Lambda \Phi+A_{3} \Phi-(2-a) H_{2} \Lambda T+A_{2} T+G_{T}
\end{aligned}
$$

with

$$
G_{T}=\partial_{\tau} G_{\Phi}-\left(H_{1}+H_{2} \frac{\Lambda Q}{Q}\right) G_{\Phi}+H_{2} \Lambda G_{\Phi}-(p-1) Q G_{\rho}
$$

and

$$
\tilde{A}_{2}=A_{1}+\left(2 a-a^{2}\right) H_{2} \Lambda H_{2}-a A_{2} H_{2} .
$$

We rewrite these equations in vectorial form

$$
\partial_{\tau} X=m X+G, \quad X=\left|\begin{array}{l}
\Phi \\
T
\end{array}, \quad G=\right| \begin{aligned}
& 0 \\
& G_{T}
\end{aligned}
$$

with

$$
m=\left(\begin{array}{cc}
-a H_{2} \Lambda & 1 \\
(p-1) Q \Delta-(1-a)^{2} H_{2}^{2} \Lambda^{2}+\tilde{A_{2}} \Lambda+A_{3}-(2-a) H_{2} \Lambda+A_{2}
\end{array}\right) .
$$

\subsection{Shifted measure}

The fine structure of the operator (3.15) involves the understanding of the associated light cone. 
Lemma 3.7 (Shifted measure) $L e t^{5}$

$$
D_{a}=(1-a)^{2}(w-1)^{2}-\sigma^{2}
$$

then for $0<a<a^{*}$ small enough, there exists a $C^{1}$ map $a \mapsto Z_{a}$ with

$$
Z_{a=0}=Z_{2}, \quad \frac{\partial Z_{a}}{\partial a}>0
$$

such that

$$
\mid \begin{aligned}
& D_{a}\left(Z_{a}\right)=0 \\
& -D_{a}(Z)>0 \text { on } 0 \leq Z<Z_{a} \\
& \lim _{Z \rightarrow 0} Z^{2}\left(-D_{a}\right)>0
\end{aligned}
$$

Proof of Lemma 3.7 We recall the notations of the Emden transform:

$$
\mid \begin{aligned}
& x=\log Z, \\
& \mu=\frac{1-e}{2}, \\
& F=\sigma+\Lambda \sigma, \\
& (p-1) Q=\mu^{2} Z^{2} \sigma^{2}, \frac{\Lambda Q}{Q}=2+2 \frac{\Lambda \sigma}{\sigma}=\frac{2(\sigma+\Lambda \sigma)}{\sigma} \\
& (p-1) \partial_{Z} Q=(p-1) \frac{\Lambda Q}{Q} \frac{Q}{Z}=2 \mu^{2} Z \sigma^{2}\left(1+\frac{\Lambda \sigma}{\sigma}\right) \\
& \quad=2 \mu^{2} Z \sigma(\sigma+\Lambda \sigma), \\
& H_{2}=\frac{1-e}{2}+2 \frac{\partial_{Z} \Psi_{P}}{Z}=\mu(1-w), \\
& H_{1}=H_{2} \frac{\Lambda \rho_{P}}{\rho_{P}}=\frac{H_{2}}{p-1} \frac{\Lambda Q}{Q}=\frac{2 \mu(\sigma+\Lambda \sigma)(1-w)}{(p-1) \sigma}, \\
& D=(w-1)^{2}-\sigma^{2} .
\end{aligned}
$$

Step 1 Values of derivatives at $P 2$. Let

$$
\Delta=(w-1)^{2}-\sigma^{2} .
$$

Let the variables

$$
w=w_{2}+W, \quad \sigma=\sigma_{2}+\Sigma,
$$

$\overline{5}$ Note that $D_{a}$ is directly connected to the highest (second) order term in $m$. Indeed, we have $(p-1) Q \partial_{Z}^{2}-(1-a)^{2} H_{2}^{2} Z^{2} \partial_{Z}^{2}=\mu^{2} Z^{2} \sigma^{2} \partial_{Z}^{2}-(1-a)^{2} \mu^{2}(1-w)^{2} Z^{2} \partial_{Z}^{2}=-\mu^{2} Z^{2} D_{a} \partial_{Z}^{2}$. 
then near $P 2$ :

$$
W=c_{-} \Sigma+O\left(\Sigma^{2}\right)
$$

Let

$$
\mid \begin{aligned}
& c_{1}=\partial_{W} \Delta_{1}\left(P_{2}\right), \\
& c_{2}=\partial_{W} \Delta_{2}\left(P_{2}\right), \\
& c_{3}=\partial_{\Sigma} \Delta_{1}\left(P_{2}\right), \\
& c_{4}=\partial_{\Sigma} \Delta_{2}\left(P_{2}\right)=-2 \sigma_{2}^{2} .
\end{aligned}
$$

Then, in our range of parameters,

$$
c_{1}<0, \quad c_{2}<0, \quad c_{3}<0, \quad c_{4}<0,
$$

and we have

$$
\mid \begin{aligned}
& c_{2} c_{-}+c_{4}=\lambda_{+} \\
& c_{2} c_{+}+c_{4}=\lambda_{-} \\
& c_{ \pm}=\frac{c_{1} c_{ \pm}+c_{3}}{c_{2} c_{ \pm}+c_{4}}
\end{aligned}
$$

which imply

$$
c_{1} c_{-}+c_{3}=c_{-}\left(c_{2} c_{-}+c_{4}\right)=c_{-} \lambda_{+}
$$

as well as

$$
-1<c_{-}<0<c_{+}, \quad \lambda_{-}<\lambda_{+}<0,
$$

see Lemmas 2.8 and 2.9 in [43].

We compute

$$
\mid \begin{aligned}
& \Delta_{1}=c_{1} W+c_{3} \Sigma+O\left(W^{2}+\Sigma^{2}\right)=\left(c_{1} c_{-}+c_{3}\right) \Sigma+O\left(\Sigma^{2}\right) \\
& \Delta_{2}=c_{2} W+c_{4} \Sigma+O\left(W^{2}+\Sigma^{2}\right)=\left(c_{2} c_{-}+c_{4}\right) \Sigma+O\left(\Sigma^{2}\right) \\
& \begin{array}{c}
\Delta=\left(1-w_{2}-W\right)^{2}-\left(\sigma_{2}+\Sigma\right)^{2}=\left(\sigma_{2}-W\right)^{2}-\left(\sigma_{2}+\Sigma\right)^{2} \\
\quad=-2 \sigma_{2}\left(c_{-}+1\right) \Sigma+O\left(\Sigma^{2}\right)
\end{array}
\end{aligned}
$$

This yields

$$
\mid \begin{aligned}
& \frac{d w}{d x}=-\frac{\Delta_{1}}{\Delta}=-\frac{c_{1} c_{-}+c_{3}+O(\Sigma)}{-2 \sigma_{2}\left(1+c_{-}\right)+O(\Sigma)}=\frac{\left|c_{-}\right|\left|\lambda_{+}\right|}{2 \sigma_{2}\left(1+c_{-}\right)}+O(\Sigma), \\
& \frac{d \sigma}{d x}=-\frac{\Delta_{2}}{\Delta}=-\frac{c_{2} c_{-}+c_{4}+O(\Sigma)}{-2 \sigma_{2}\left(1+c_{-}\right)+O(\Sigma)}=-\frac{\left|\lambda_{+}\right|}{2 \sigma_{2}\left(1+c_{-}\right)}+O(\Sigma),
\end{aligned}
$$

and hence

$$
Z_{2} \frac{d \Delta}{d Z}\left(Z_{2}\right)=\frac{d \Delta}{d x}\left(P_{2}\right)=-2\left(1-w_{2}\right) \frac{d w}{d x}\left(P_{2}\right)-2 \sigma_{2} \frac{d \sigma}{d x}\left(P_{2}\right)
$$




$$
\begin{aligned}
& =-2 \sigma_{2} \frac{\left|c_{-}\right|\left|\lambda_{+}\right|}{2 \sigma_{2}\left(1+c_{-}\right)}-2 \sigma_{2}\left[-\frac{\left|\lambda_{+}\right|}{2 \sigma_{2}\left(1+c_{-}\right)}\right] \\
& =\frac{\left|\lambda_{+}\right|}{1+c_{-}}\left(1-\left|c_{-}\right|\right) \\
& =\left|\lambda_{+}\right|>0
\end{aligned}
$$

Step 2 Computation of $Z_{a}$. Let $D_{0}(Z)=\Delta(Z)$, we have $D_{0}^{\prime}\left(Z_{2}\right)>0$ from (3.24) and hence by the implicit function theorem applied to the function $F(a, Z)=D_{a}(Z)$ at $(a, Z)=\left(0, Z_{2}\right)$ where $D_{0}\left(Z_{2}\right)=0$, we infer for all $a$ small enough the existence of a locally unique solution $Z_{a}$ to

$$
D_{a}\left(Z_{a}\right)=0 .
$$

Furthermore, $Z_{a}$ is $C^{1}$ in a neighborhood of $a=0$ and its derivative is given by

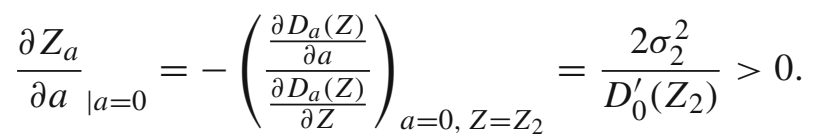

Thus

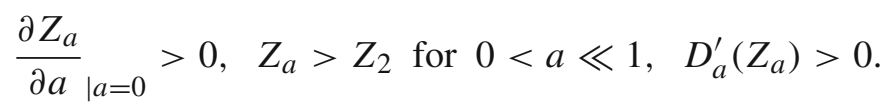

We now observe

$$
D_{a}(Z)=((1-a)(1-w)+\sigma)((1-a)(1-w)-\sigma)
$$

so that $D_{a}(Z)$ is of the sign of $(1-a)(1-w)-\sigma$ since $w<1$ and $\sigma>0$. Now from (3.23):

$$
\begin{aligned}
\frac{d}{d x}((1-a)(1-w)-\sigma) & =-(1-a) \frac{\left|c_{-}\right|\left|\lambda_{+}\right|}{2 \sigma_{2}\left(1+c_{-}\right)}+\frac{\left|\lambda_{+}\right|}{2 \sigma_{2}\left(1+c_{-}\right)} \\
& =\frac{\left|\lambda_{+}\right|}{2 \sigma_{2}\left(1+c_{-}\right)}\left[1-(1-a)\left|c_{-}\right|\right]>0 .
\end{aligned}
$$

Thus, $(1-a)(1-w)-\sigma$ is increasing on $\left(0, Z_{a}\right]$ and vanishes at $Z=Z_{a}$ so that

$$
D_{a}(Z)<0 \text { on }\left(0, Z_{a}\right)
$$

Moreover, we have in view of the behavior of $\sigma$ and $w$ as $Z \rightarrow 0_{+}$, see Lemma 3.1 in [43],

$$
\lim _{Z \rightarrow 0_{+}} Z^{2}\left(-D_{a}(Z)\right)=\lim _{Z \rightarrow 0_{+}} Z^{2} \sigma^{2}=1>0
$$


This concludes the proof of (3.17).

\subsection{Commuting with derivatives}

We define

$$
T_{k}=\Delta^{k} T, \quad \Phi_{k}=\Delta^{k} \Phi .
$$

Lemma 3.8 (Commuting with derivatives) Let $k \in \mathbb{N}$. There exists a smooth measure $^{6} g$ defined for $Z \in\left[0, Z_{a}\right]$ such that the following holds. Let the elliptic operator

$$
\mathcal{L}_{g} \Phi_{k}=\frac{\mu^{2}}{g Z^{d-1}} \partial_{Z}\left(Z^{d-1} Z^{2} g\left(-D_{a}\right) \partial_{Z} \Phi_{k}\right),
$$

then there holds

$$
\Delta^{k}(m X)=m_{k} \mid \begin{aligned}
& \Phi_{k} \\
& T_{k}
\end{aligned}+\tilde{m}_{k} X
$$

with

$m_{k}\left|\begin{array}{l}\Phi_{k} \\ T_{k}\end{array}=\right| \begin{aligned} & -a H_{2} \Lambda \Phi_{k}-2 a k\left(H_{2}+\Lambda H_{2}\right) \Phi_{k}+T_{k} \\ & \mathcal{L}_{g} \Phi_{k}-(2-a) H_{2} \Lambda T_{k}-2 k(2-a)\left(H_{2}+\Lambda H_{2}\right) T_{k}+A_{2} T_{k}\end{aligned}$,

where $\tilde{m}_{k}$ satisfies the following pointwise bound

$$
\left|\tilde{m}_{k} X\right| \lesssim_{k} \mid \begin{aligned}
& \sum_{j=0}^{2 k-1}\left|\partial_{Z}^{j} \Phi\right|, \\
& \sum_{j=0}^{2 k}\left|\partial_{Z}^{j} \Phi\right|+\sum_{j=0}^{2 k-1}\left|\partial_{Z}^{j} T\right| .
\end{aligned}
$$

Moreover, $g>0$ in $\left[0, Z_{a}\right)$ and admits the asymptotics:

$$
\mid \begin{aligned}
& g(Z)=1+O\left(Z^{2}\right) \text { as } Z \rightarrow 0, \\
& g(Z)=c_{a, d, r, \ell}\left(Z_{a}-Z\right)^{c_{g}}\left[1+O\left(Z-Z_{a}\right)\right] \text { as } Z \uparrow Z_{a},
\end{aligned}
$$

with $c_{a, d, r, \ell}>0$ and

$$
c_{g}>0
$$

\footnotetext{
$\overline{6} g$ is the density of the measure $g Z^{d-1} d Z$ with respect to which the operator $\mathcal{L}_{g}$ is selfadjoint. By a slight abuse of terminology, we call $g$ a measure. 
for all $k \geq k_{1}$ large enough and $0<a<a^{*}$ small enough. Finally, note that $g$ and $c_{g}$ depend on $k$.

Proof This is a direct computation.

Step 1 Proof of (3.27), (3.28). We recall (C.1):

$$
\left[\Delta^{k}, V\right] \Phi-2 k \nabla V \cdot \nabla \Delta^{k-1} \Phi=\sum_{|\alpha|+|\beta|=2 k,|\beta| \leq 2 k-2} c_{k, \alpha, \beta} \partial^{\alpha} V \partial^{\beta} \Phi,
$$

which together with the commutator formulas

$$
\mid \begin{aligned}
& {\left[\Delta^{k}, \Lambda\right]=2 k \Delta^{k}, \quad\left[\partial_{Z}, \Lambda\right]=\partial_{Z}} \\
& \Lambda^{2}=Z^{2} \Delta-(d-2) \Lambda \\
& \partial_{Z} \Lambda=\frac{\Lambda^{2}}{Z}=Z \Delta-(d-2) \partial_{Z}
\end{aligned}
$$

yields

$$
\begin{aligned}
\Delta^{k}(V \Lambda \Phi)= & V \Delta^{k}(\Lambda \Phi)+2 k \nabla V \cdot \nabla \Delta^{k-1} \Lambda \Phi \\
& +\sum_{|\alpha|+|\beta|=2 k,|\beta| \leq 2 k-2} c_{k, \alpha, \beta} \partial^{\alpha} V \partial^{\beta} \Lambda \Phi
\end{aligned}
$$

and

$$
\begin{aligned}
& 2 k \nabla V \cdot \nabla \Delta^{k-1} \Lambda \Phi=2 k \partial_{Z} V \partial_{Z}\left[\Lambda \Delta^{k-1} \Phi+2(k-1) \Phi_{k-1}\right] \\
& \quad=2 k \partial_{Z} V\left[\left(Z \Delta-(d-2) \partial_{Z}\right) \Phi_{k-1}+2(k-1) \partial_{Z} \Phi_{k-1}\right] \\
& =2 k \Lambda V \Phi_{k}+2 k(2 k-2-d+2) \partial_{Z} V \partial_{Z} \Phi_{k-1}
\end{aligned}
$$

from which for $0 \leq Z \leq Z_{a}$ :

$$
\Delta^{k}(V \Lambda \Phi)=V(2 k+\Lambda) \Phi_{k}+2 k \Lambda V \Phi_{k}+O_{k, a}\left(\sum_{j=0}^{2 k-1}\left|\partial_{Z}^{j} \Phi\right|\right) .
$$

We then use

$$
\begin{aligned}
{\left[\Delta^{k}, \Lambda^{2}\right] } & =\Delta^{k} \Lambda^{2}-\Lambda^{2} \Delta^{k}=\left[\Delta^{k}, \Lambda\right] \Lambda+\Lambda \Delta^{k} \Lambda-\Lambda\left(-\left[\Delta^{k}, \Lambda\right]+\Delta^{k} \Lambda\right) \\
& =2 k \Delta^{k} \Lambda+2 k \Lambda \Delta^{k}=4 k^{2} \Delta^{k}+4 k \Lambda \Delta^{k}
\end{aligned}
$$

to compute similarly:

$$
\Delta^{k}\left(V \Lambda^{2} \Phi\right)=V \Delta^{k}\left(\Lambda^{2} \Phi\right)+2 k \nabla V \cdot \nabla \Delta^{k-1} \Lambda^{2} \Phi
$$




$$
\begin{aligned}
+ & \sum_{|\alpha|+|\beta|=2 k,|\beta| \leq 2 k-2} c_{k, \alpha, \beta} \partial^{\alpha} V \partial^{\beta} \Lambda \Phi \\
= & V\left[\Lambda^{2} \Phi_{k}+4 k^{2} \Phi_{k}+4 k \Lambda \Phi_{k}\right] \\
& +2 k \partial_{Z} V \partial_{Z} \Delta^{k-1} \Lambda^{2} \Phi+O\left(\sum_{j=0}^{2 k}\left|\partial_{Z}^{j} \Phi\right|\right)
\end{aligned}
$$

and

$$
\begin{aligned}
\partial_{Z} \Delta^{k-1} \Lambda^{2} \Phi & =\partial_{Z}\left[\Lambda^{2} \Phi_{k-1}+4(k-1)^{2} \Phi_{k-1}+4(k-1) \Lambda \Phi_{k-1}\right] \\
& =\partial_{Z}\left(Z^{2} \Phi_{k}-(d-2) \Lambda \Phi_{k-1}\right)+O\left(\sum_{j=0}^{2 k}\left|\partial_{Z}^{j} \Phi\right|\right) \\
& =Z \Lambda \Phi_{k}+O\left(\sum_{j=0}^{2 k}\left|\partial_{Z}^{j} \Phi\right|\right)
\end{aligned}
$$

and hence

$$
\begin{aligned}
\Delta^{k}\left(V \Lambda^{2} \Phi\right)= & V\left[\Lambda^{2} \Phi_{k}+4 k^{2} \Phi_{k}+4 k \Lambda \Phi_{k}\right] \\
& +2 k \Lambda V \Lambda \Phi_{k}+O\left(\sum_{j=0}^{2 k}\left|\partial_{Z}^{j} \Phi\right|\right) .
\end{aligned}
$$

Recalling the definition of the operator (3.15), we obtain (3.27), (3.28) with

$m_{k}\left|\begin{array}{l|l}\Phi_{k} \\ T_{k}\end{array}=\right| \begin{aligned} & -a H_{2} \Lambda \Phi_{k}-2 a k\left(H_{2}+\Lambda H_{2}\right) \Phi_{k}+T_{k} \\ & (p-1) Q \Delta \Phi_{k}-(1-a)^{2} H_{2}^{2} \Lambda^{2} \Phi_{k}+A_{k} \Lambda \Phi_{k}-(2-a) H_{2} \Lambda T_{k} \\ & -2 k(2-a)\left(H_{2}+\Lambda H_{2}\right) T_{k}+A_{2} T_{k}\end{aligned}$

and

$$
A_{k}=2 k(p-1) \frac{\partial_{Z} Q}{Z}-(1-a)^{2} 4 k H_{2}\left[H_{2}+\Lambda H_{2}\right]+\tilde{A}_{2} .
$$

Step 2 Equation for the measure. We compute using (3.18), (3.16):

$$
\begin{aligned}
& (p-1) Q \Delta \Phi_{k}-(1-a)^{2} H_{2}^{2} \Lambda^{2} \Phi_{k} \\
& =\mu^{2} Z^{2} \sigma^{2}\left(\partial_{Z}^{2} \Phi_{k}+\frac{d-1}{Z} \partial_{Z} \Phi_{k}\right) \\
& \quad-\mu^{2}(1-w)^{2}(1-a)^{2}\left(Z^{2} \partial_{Z}^{2} \Phi_{k}+\Lambda \Phi_{k}\right)
\end{aligned}
$$




$$
=\mu^{2}\left\{-Z^{2} D_{a} \partial_{Z}^{2} \Phi_{k}+Z \partial_{Z} \Phi_{k}\left[(d-1) \sigma^{2}-(1-a)^{2}(1-w)^{2}\right]\right\}
$$

and, using $F=\sigma+\Lambda \sigma$,

$$
\begin{aligned}
A_{k} & =2 k(p-1) \frac{\partial_{Z} Q}{Z}-(1-a)^{2} 4 k H_{2}\left[H_{2}+\Lambda H_{2}\right]+\tilde{A}_{2} \\
& =4 k \mu^{2}\left[\sigma F-(1-a)^{2}(1-w)+(1-a)^{2}(1-w)(w+\Lambda w)\right]+\tilde{A}_{2}
\end{aligned}
$$

and hence:

$$
\begin{aligned}
& (p-1) Q \Delta \Phi_{k}-(1-a)^{2} H_{2}^{2} \Lambda^{2} \Phi_{k}+A_{k} \Lambda \Phi_{k} \\
& =-\mu^{2} Z^{2} D_{a} \partial_{Z}^{2} \Phi_{k}+\Lambda \Phi_{k}\left[\mu^{2}\left((d-1) \sigma^{2}-(1-a)^{2}(1-w)^{2}\right)\right. \\
& \left.\quad+4 k \mu^{2}\left[\sigma F-(1-a)^{2}(1-w)+(1-a)^{2}(1-w)(w+\Lambda w)\right]+\tilde{A}_{2}\right] .
\end{aligned}
$$

We compute the measure

$$
\begin{aligned}
& \frac{\mu^{2}}{g Z^{d-1}} \partial_{Z}\left(Z^{d-1} Z^{2} g\left(-D_{a}\right) \Phi_{k}^{\prime}\right) \\
& =\mu^{2} Z^{2}\left(-D_{a}\right) \partial_{Z}^{2} \Phi_{k}-\mu^{2} \Lambda \Phi_{k}\left((d+1) D_{a}+\frac{g^{\prime}}{g} Z D_{a}+Z D_{a}^{\prime}\right)
\end{aligned}
$$

and hence the relation:

$$
\begin{aligned}
- & \mu^{2}\left((d+1) D_{a}+\frac{g^{\prime}}{g} Z D_{a}+Z D_{a}^{\prime}\right) \\
= & \mu^{2}\left((d-1) \sigma^{2}-(1-w)^{2}\right)+4 k \mu^{2}\left[\sigma F-(1-a)^{2}(1-w)\right. \\
& \left.+(1-a)^{2}(1-w)(w+\Lambda w)\right]+\tilde{A}_{2} .
\end{aligned}
$$

Equivalently:

$$
\left(-D_{a}\right) \frac{\Lambda g}{g}=-C_{\mathcal{L}}
$$

with

$$
\begin{aligned}
\mathcal{L}= & -(d-1) \sigma^{2}+(1-w)^{2}-(d+1) D_{a}-\Lambda D_{a} \\
& +4 k\left[(1-a)^{2}(1-w)-\sigma F-(1-a)^{2}(1-w)(w+\Lambda w)\right] \\
& -\frac{\tilde{A}_{2}}{\mu^{2}}
\end{aligned}
$$


Step 3 Asymptotics of the measure. We now solve (3.32). Near the origin, the normalization (2.17) and (3.18) yield

$\sigma=\frac{\sqrt{p-1}}{\mu Z}\left[1+O\left(Z^{2}\right)\right], \quad F=\sigma+\Lambda \sigma=O(Z), \quad-D_{a}=\sigma^{2}+O(1)$

We compute

$$
\frac{\tilde{A_{2}}}{\mu^{2}}=O\left(\frac{|F|}{\sigma}+|\Lambda w|+|a|\right)=O(1)
$$

and hence

$$
\begin{aligned}
\mathcal{L}= & -(d-1) \sigma^{2}-(d+1)\left(-\sigma^{2}\right)-\Lambda\left(-\sigma^{2}\right) \\
& +O(1+|a|+\sigma|F|+|w|+|\Lambda w|) \\
= & 2 \sigma F+O(1)=O(1)
\end{aligned}
$$

which, recalling (3.17), yields:

$$
-\frac{\mathcal{L}}{\left(-D_{a}\right)}=\frac{O(1)}{\sigma^{2}+O(1)}=O\left(Z^{2}\right)
$$

and we may therefore choose explicitly:

$$
g=e^{\int_{0}^{Z}\left[-\frac{Q}{(-D a)}\right] \frac{d \tau}{\tau}}
$$

To compute the behavior near $Z_{a}$, recall from (3.25) (3.26) that we have

$$
D_{a}\left(Z_{a}\right)=0, \quad D_{a}^{\prime}\left(Z_{a}\right)>0
$$


We infer in the neighborhood of $Z=Z_{a}$

$$
\begin{aligned}
\frac{\partial_{Z} g}{g} & =\frac{\mathcal{L}}{Z D_{a}}=\frac{\mathcal{L}\left(Z_{a}\right)}{\Lambda D_{a}\left(Z_{a}\right)} \frac{1+O\left(Z-Z_{a}\right)}{Z-Z_{a}} \\
& =\left(\frac{\mathcal{L}_{(}\left(Z_{2}\right)}{\Lambda D_{0}\left(Z_{2}\right)}+O(|a|)\right) \frac{1+O\left(Z-Z_{a}\right)}{Z-Z_{a}} .
\end{aligned}
$$

The fundamental computation is then at $P 2$ using (3.23):

$$
\begin{aligned}
& {[(1-w)-\sigma(\sigma+\Lambda \sigma)-(1-w)(w+\Lambda w)]} \\
& \quad=\left(1-w_{2}\right)\left(1-w_{2}-\Lambda w\right)-\sigma_{2}\left(\sigma_{2}+\Lambda \sigma\right) \\
& \quad=\sigma_{2}\left(\sigma_{2}-\Lambda w\right)-\sigma_{2}\left(\sigma_{2}+\Lambda \sigma\right)=\sigma_{2}(-\Lambda w-\Lambda \sigma) \\
& \quad=\sigma_{2}\left[-\frac{\left|c_{-}\right|\left|\lambda_{+}\right|}{2 \sigma_{2}\left(1+c_{-}\right)}+\frac{\left|\lambda_{+}\right|}{2 \sigma_{2}\left(1+c_{-}\right)}\right]=\frac{\left|\lambda_{+}\right|}{2}>0 .
\end{aligned}
$$

Hence from (3.33)

$$
\mathcal{L}_{2}\left(Z_{2}\right)=2 k\left(\left|\lambda_{+}\right|+O(a)\right)+O(1)
$$

and from (3.24)

$$
\frac{\mathcal{L}_{(}\left(Z_{2}\right)}{\Lambda D_{0}\left(Z_{2}\right)}=\frac{2 k\left(\left|\lambda_{+}\right|+O(a)\right)+O(1)}{\left|\lambda_{+}\right|}>k
$$

for $0<a<a^{*}$ small enough and $k \geq k_{1}$ large enough. Inserting this into (3.34) yields (3.29).

\subsection{Hardy inequality and compactness}

We let $k \geq k_{1}$ large enough so that (3.30) holds and extend the measure $g$ by zero for $Z \geq Z_{a}$. We let $\chi$ be a smooth cut off function supported strictly inside the light cone $|Z|<Z_{2}$ with

$$
g \geq \frac{1}{2} \text { on } \operatorname{Supp} \chi
$$

Let

$$
\mathscr{D}_{\Phi}=\left\{\Phi \in e^{\infty}\left(\left[0, Z_{a}\right], \mathbb{C}\right) \text { with spherical symmetry }\right\}
$$

be the space of test functions and

$$
\langle\langle\Phi, \tilde{\Phi}\rangle\rangle=-\left(\mathcal{L}_{g} \Phi_{k}, \tilde{\Phi}_{k}\right)_{g}+\int \chi \Phi \overline{\tilde{\Phi}} g Z^{d-1} d Z
$$


be a Hermitian scalar product, where we recall the notation (1.13). We let $\mathbb{H}_{\Phi}$ be the completion of $\mathscr{D}_{\Phi}$ for the norm associated to (3.35). We claim the following compactness subcoercivity estimate:

Lemma 3.9 (Subcoercivity estimate) For $0<v<1$ :

$$
\begin{aligned}
\langle\langle\Phi, \Phi\rangle\rangle \gtrsim & \int \frac{\left|\Phi_{k}\right|^{2}}{Z_{a}-Z} g Z^{d-1} d Z \\
& +\sum_{m=0}^{2 k} \int\left|\partial_{Z}^{m} \Phi(Z)\right|^{2} \frac{g}{\left(Z_{a}-Z\right)^{1-\nu}} Z^{d-1} d Z .
\end{aligned}
$$

Furthermore, there exists $c>0$ and a sequence $\mu_{n}>0$ with $\lim _{n \rightarrow+\infty} \mu_{n}=$ $+\infty$ and $\Pi_{n} \in \mathbb{H}_{\Phi}, c_{n}>0$ such that $\forall n \geq 0, \forall \Phi \in \mathbb{H}_{\Phi}$,

$$
\begin{aligned}
\langle\langle\Phi, \Phi\rangle\rangle \geq & c \int \frac{\left|\Phi_{k}\right|^{2}}{Z_{a}-Z} g Z^{d-1} d Z \\
& +\mu_{n} \sum_{m=0}^{2 k} \int\left|\partial_{Z}^{m} \Phi(Z)\right|^{2} \frac{g}{\left(Z_{a}-Z\right)^{1-\nu}} Z^{d-1} d Z \\
& -c_{n} \sum_{i=1}^{n}\left(\Phi, \Pi_{i}\right)_{g}^{2} .
\end{aligned}
$$

Proof This is a classical Hardy and Sobolev based argument with a loss $v$. We provide a proof for the reader's convenience.

Step 1 Interior estimate. Let $Z_{0}<Z_{a}$ which will be chosen close enough to $Z_{a}$ in step 2. Then, we have

$$
\begin{aligned}
& \int_{0}^{Z_{0}} \frac{\left|\Phi_{k}\right|^{2}}{Z_{a}-Z} g Z^{d-1} d Z+\sum_{m=0}^{2 k} \int_{0}^{Z_{0}}\left|\partial_{Z}^{m} \Phi(Z)\right|^{2} \frac{g}{\left(Z_{a}-Z\right)^{1-\nu}} Z^{d-1} d Z \\
& \quad \leq C_{Z_{0}}\|\Phi\|_{H^{2 k}\left(0, Z_{0}\right)}^{2} \\
& \quad \leq C_{Z_{0}}\left[\int_{0}^{Z_{0}}\left|\partial_{Z} \Phi_{k}\right|^{2} Z^{d-1} d Z+\int_{0}^{Z_{0}} \chi|\Phi(Z)|^{2} Z^{d-1} d Z\right] .
\end{aligned}
$$

Since $-Z^{2} D_{a}$ and $g$ are smooth and satisfy $-Z^{2} D_{a}>0$ and $g>0$ on $\left[0, Z_{0}\right]$, we infer 


$$
\begin{aligned}
\langle\langle\Phi, \Phi\rangle\rangle \geq & c_{Z_{0}}\left[\int_{0}^{Z_{0}} \frac{\left|\Phi_{k}\right|^{2}}{Z_{a}-Z} g Z^{d-1} d Z\right. \\
& \left.+\sum_{m=0}^{2 k} \int_{0}^{Z_{0}}\left|\partial_{Z}^{m} \Phi(Z)\right|^{2} \frac{g}{\left(Z_{a}-Z\right)^{1-v}} Z^{d-1} d Z\right]
\end{aligned}
$$

for some $c_{Z_{0}}>0$. Thus, to prove (3.36), it remains to consider the region $\left(Z_{0}, Z_{a}\right)$. This will be done in steps 2 and 3 .

Step 2 Hardy inequality with loss. Let $0<v<1$, we claim the lossy Hardy bound for all $\Phi \in \mathscr{D}_{\Phi}$ :

$$
\sum_{m=0}^{2 k} \int_{Z_{0}}^{Z_{a}}\left|\partial_{Z}^{m} \Phi(Z)\right|^{2} \frac{g}{\left(Z_{a}-Z\right)^{1-v}} Z^{d-1} d Z \leq c_{v}\langle\langle\Phi, \Phi\rangle\rangle .
$$

Indeed, let $Z_{0}=Z_{a}-\delta$ with $\delta>0$ small enough, we estimate by Taylor expansion for $Z_{0} \leq Z<Z_{a}$ for $0 \leq m \leq 2 k$ :

$$
\left|\partial_{Z}^{m} \Phi(Z)\right|^{2} \leq C\left[\sum_{j=m}^{2 k}\left|\partial_{Z}^{j} \Phi\left(Z_{0}\right)\right|^{2}+\left(\int_{Z_{0}}^{Z}\left|\partial_{Z}^{2 k+1} \Phi(\tau)\right| d \tau\right)^{2}\right]
$$

From Sobolev,

$$
\sum_{j=m}^{2 k}\left|\partial_{Z}^{j} \Phi\left(Z_{0}\right)\right|^{2} \leq C_{Z_{0}}\|\Phi\|_{H^{2 k+1}\left(0, Z_{0}\right)}^{2} \leq C_{Z_{0}}\langle\langle\Phi, \Phi\rangle\rangle
$$

and hence

$$
\begin{aligned}
\left|\partial_{Z}^{m} \Phi(Z)\right|^{2} \leq & C_{Z_{0}}\langle\langle\Phi, \Phi\rangle\rangle+C\left(\int_{Z_{0}}^{Z}\left|\partial_{Z} \Delta^{k} \Phi(\tau)\right| d \tau\right)^{2} \\
& +C\left(\int \sum_{j=1}^{2 k}\left|\partial_{Z}^{j} \Phi\right| d \tau\right)^{2} \\
\leq & C_{Z_{0}}\langle\langle\Phi, \Phi\rangle\rangle+C\left(\int_{Z_{0}}^{Z}\left|\partial_{Z} \Phi_{k}\right|^{2}\left(Z_{a}-Z\right)^{1-v} Z^{d-1} d Z\right) \\
& \times\left(\int_{Z_{0}}^{Z} \frac{d \tau}{\left(Z_{a}-\tau\right)^{1-v}}\right)^{2 k}\left(\int_{Z_{0}}^{Z} \frac{\left|\partial_{Z}^{j} \Phi\right|^{2}}{\left(Z_{a}-Z\right)^{1-v}} d Z\right)\left(\int_{Z_{0}}^{Z}\left(Z_{a}-\tau\right)^{1-v} d \tau\right) \\
& +C \sum_{j=1}\left(\int^{1-1}\right)
\end{aligned}
$$




$$
\begin{aligned}
\leq & C_{Z_{0}}\langle\langle\Phi, \Phi\rangle\rangle+C \delta^{\nu} \int_{Z_{0}}^{Z}\left|\partial_{Z} \Phi_{k}\right|^{2}\left(Z_{a}-\tau\right)^{1-v} d \tau \\
& +C \delta \sum_{j=m}^{2 k}\left(\int_{Z_{0}}^{Z} \frac{\left|\partial_{Z}^{j} \Phi\right|^{2}}{\left(Z_{a}-\tau\right)^{1-\nu}} d \tau\right),
\end{aligned}
$$

where we used the fact that $0<v<1$ and $Z_{a}-Z_{0}=\delta$. Using again $0<v<1$ and $Z_{a}-Z_{0}=\delta$, as well as Fubini and the fact that $\partial_{Z} g<0$ on $\left(Z_{0}, Z_{a}\right)$ for $Z_{0}$ close enough to $Z_{a}$ so that $g$ is decreasing on $\left(Z_{0}, Z_{a}\right)$, we infer

$$
\begin{aligned}
& \sum_{m=0}^{2 k} \int_{Z_{0}}^{Z_{a}}\left|\partial_{Z}^{m} \Phi(Z)\right|^{2} \frac{g}{\left(Z_{a}-Z\right)^{1-\nu}} Z^{d-1} d Z \\
& \leq C_{Z_{0}}\langle\langle\Phi, \Phi\rangle\rangle+C \delta^{v} \int_{Z_{0}}^{Z_{a}} \frac{g}{\left(Z_{a}-Z\right)^{1-v}} \\
& \times\left(\int_{Z_{0}}^{Z}\left|\partial_{Z} \Phi_{k}\right|^{2}\left(Z_{a}-\tau\right)^{1-v} d \tau\right) Z^{d-1} d Z \\
& +C \delta \sum_{j=0}^{2 k} \int_{Z_{0}}^{Z_{a}} \frac{g}{\left(Z_{a}-Z\right)^{1-\nu}}\left(\int_{Z_{0}}^{Z} \frac{\left|\partial_{Z}^{j} \Phi\right|^{2}}{\left(Z_{a}-\tau\right)^{1-\nu}} d \tau\right) Z^{d-1} d Z \\
& \leq C_{Z_{0}}\langle\langle\Phi, \Phi\rangle\rangle+C \delta^{\nu} \int_{Z_{0}}^{Z_{a}}\left|\partial_{Z} \Phi_{k}\right|^{2} g(\tau)\left(Z_{a}-\tau\right)^{1-\nu} \\
& \times\left(\int_{\tau}^{Z_{a}} \frac{d Z}{\left(Z_{a}-Z\right)^{1-v}}\right) \tau^{d-1} d \tau \\
& +C \delta \sum_{j=0}^{2 k} \int_{Z_{0}}^{Z_{a}} \frac{\left|\partial_{Z}^{j} \Phi\right|^{2} g(\tau)}{\left(Z_{a}-\tau\right)^{1-\nu}}\left(\int_{\tau}^{Z_{a}} \frac{d Z}{\left(Z_{a}-Z\right)^{1-\nu}}\right) \tau^{d-1} d \tau \\
& \leq C_{Z_{0}}\langle\langle\Phi, \Phi\rangle\rangle+C \delta^{\nu} \int_{Z_{0}}^{Z_{a}}\left|\partial_{Z} \Phi_{k}\right|^{2} g(\tau)\left(Z_{a}-\tau\right) \tau^{d-1} d \tau \\
& +C \delta \sum_{j=0}^{2 k} \int_{Z_{0}}^{Z_{a}} \frac{\left|\partial_{Z}^{j} \Phi\right|^{2} g(\tau)}{\left(Z_{a}-\tau\right)^{1-\nu}} \tau^{d-1} d \tau .
\end{aligned}
$$

Letting $\delta=\delta(v)$ small enough and estimating from (3.26)

$$
-\left(D_{a}\right)(Z) \geq c\left(Z_{a}-Z\right)
$$

yields (3.38). 
Step 3 Sharp Hardy. We now claim the sharp Hardy inequality for $f \in \mathscr{D}_{\Phi}$ :

$$
\langle\langle\Phi, \Phi\rangle\rangle \gtrsim \int_{Z_{0}}^{Z_{a}} \frac{\left|\Phi_{k}\right|^{2}}{\left(Z_{a}-Z\right)} g Z^{d-1} d Z
$$

Indeed, recall (3.29), (3.30) near $Z_{a}$ :

$$
g(Z)=c\left(Z_{a}-Z\right)^{c_{g}}\left[1+O\left(Z-Z_{a}\right)\right],
$$

then integrating by parts:

$$
\begin{aligned}
& \int_{Z_{0}}^{Z_{a}} \frac{\left|\Phi_{k}\right|^{2}}{\left(Z_{a}-Z\right)} g Z^{d-1} d Z \lesssim \int_{Z_{0}}^{Z_{a}}\left|\Phi_{k}\right|^{2}\left(Z_{a}-Z\right)^{c_{g}-1} d Z \\
& =-\frac{1}{c_{g}}\left[\left|\Phi_{k}\right|^{2}\left(Z_{a}-Z\right)^{c_{g}}\right]_{Z_{0}}^{Z_{a}}+\frac{1}{c_{g}} \int_{Z_{0}}^{Z_{a}} 2 \Phi_{k} \partial_{Z} \Phi_{k}\left(Z_{a}-Z\right)^{c_{g}} d Z \\
& \lesssim\left|\Phi_{k}\right|^{2}\left(Z_{0}\right)+\left(\int\left|\Phi_{k}\right|^{2}\left(Z_{a}-Z\right)^{c_{g}-1} Z^{d-1} d Z\right)^{\frac{1}{2}} \\
& \quad \times\left(\int\left|\partial_{Z} \Phi_{k}\right|^{2}\left(Z_{a}-Z\right)^{c_{g}+1} Z^{d-1} d Z\right)^{\frac{1}{2}} \\
& \lesssim\langle\langle\Phi, \Phi\rangle\rangle+\left(\int_{Z_{0}}^{Z_{a}} \frac{\left|\Phi_{k}\right|^{2}}{\left(Z_{a}-Z\right)} g Z^{d-1} d Z\right)^{\frac{1}{2}} \\
& \quad \times\left(\int\left|\partial_{Z} \Phi_{k}\right|^{2} g\left(-D_{a}\right) Z^{d-1} d Z\right)^{\frac{1}{2}},
\end{aligned}
$$

where we used (3.39). The bound (3.40) now follows using Hölder. Together with steps 1 and 2, this concludes the proof of (3.36).

Step 4 Compactness. We now turn to the proof of (3.37) which follows from a standard compactness argument. Let us consider $T \in L_{g}^{2}$. Then from (3.36), the antilinear form

$$
h \mapsto(T, h)_{g}
$$

is continuous on $\mathbb{H}_{\Phi}$, and hence by Riesz Theorem, there exists a unique $L(T) \in \mathbb{H}_{\Phi}$ such that

$$
\forall h \in \mathbb{H}_{\Phi}, \quad\langle\langle L(T), h\rangle\rangle=(T, h)_{g},
$$


and the linear map $L$ is bounded from $L_{g}^{2}$ to $\mathbb{H}_{\Phi}$. For any $0<\delta<Z_{a}$, we have in view of (3.36)

$$
\begin{aligned}
\|h\|_{L_{g}^{2}} & \leq \delta^{\frac{1-v}{2}}\left\|\frac{h}{\left(Z_{a}-Z\right)^{\frac{1-v}{2}}}\right\|_{L_{g}^{2}}+\|h\|_{L_{g}^{2}\left(Z \leq Z_{a}-\delta\right)} \\
& \lesssim \delta^{\frac{1-v}{2}}\|h\|_{\mathbb{H}_{\Phi}}+\|h\|_{L_{g}^{2}\left(Z \leq Z_{a}-\delta\right)} .
\end{aligned}
$$

Relying on the smallness of $\delta^{\frac{1-v}{2}}$ for the first term, and Rellich Theorem for the second one, we easily infer that

$$
\mathbb{H}_{\Phi} \text { embeds compactly in } L_{g}^{2} \text {. }
$$

Since $L$ is bounded from $L_{g}^{2}$ to $\mathbb{H}_{\Phi}$, we infer that the map

$$
L: L_{g}^{2} \mapsto L_{g}^{2}
$$

is compact. Moreover, if $\Phi_{1}=L\left(T_{1}\right), \Phi_{2}=L\left(T_{2}\right)$ :

$$
\left(L\left(T_{1}\right), T_{2}\right)_{g}=\left(\Phi_{1}, T_{2}\right)_{g}=\overline{\left(T_{2}, \Phi_{1}\right)_{g}}=\overline{\left\langle\left\langle L T_{2}, \Phi_{1}\right\rangle\right\rangle}=\left\langle\left\langle\Phi_{1}, \Phi_{2}\right\rangle\right\rangle
$$

and hence interchanging the roles of $T_{1}, T_{2}$ :

$$
\left(T_{1}, L\left(T_{2}\right)\right)_{g}=\overline{\left(L\left(T_{2}\right), T_{1}\right)_{g}}=\overline{\left\langle\left\langle\Phi_{2}, \Phi_{1}\right\rangle\right\rangle}=\left\langle\left\langle\Phi_{1}, \Phi_{2}\right\rangle\right\rangle=\left(L\left(T_{1}\right), T_{2}\right)_{g}
$$

and $L$ is selfadjoint on $L_{g}^{2}$. Since $L>0$ from (3.41), we conclude that $L$ is a diagonalizable with a non increasing sequences of eigenvalues $\lambda_{n}>0$, $\lim _{n \rightarrow+\infty} \lambda_{n}=0$, and let $\left(\Pi_{n, i}\right)_{1 \leq i \leq I(n)}$ be an $L_{g}^{2}$ orthonormal basis for the eigenvalue $\lambda_{n}$. The eigenvalue equation implies $\Pi_{n, i} \in \mathbb{H}_{\Phi}$.

Let then

$$
\begin{aligned}
\mathcal{A}_{n}= & \left\{\Phi \in \mathbb{H}_{\Phi}, \quad \int|\Phi|^{2} g Z^{d-1} d Z=1,\left(\Phi, \Pi_{j, i}\right)_{g}=0, \quad 1 \leq i \leq I(j),\right. \\
1 \leq j \leq n\} &
\end{aligned}
$$

and the minimization problem

$$
I_{n}=\inf _{\Phi \in \mathcal{A}_{n}}\langle\langle\Phi, \Phi\rangle\rangle,
$$


then the infimum is attained in view of (3.42) at $\Phi \in \mathcal{A}_{n}$ and, by a standard Lagrange multiplier argument:

$$
\forall h \in \mathbb{H}_{\Phi}, \quad\langle\langle\Phi, h\rangle\rangle=\sum_{j=1}^{n} \sum_{i=1}^{I(j)} \alpha_{i, j}\left(\Pi_{j, i}, h\right)_{g}+\alpha(\Phi, h)_{g} .
$$

Letting $h=\Pi_{i, j}$ implies $\alpha_{i, j}=0$ and hence from (3.41):

$$
L(\Phi)=\frac{1}{\alpha} \Phi,
$$

which together with our orthogonality conditions implies

$$
\frac{1}{\alpha} \leq \lambda_{n+1}
$$

and hence

$$
I_{n}=\langle\langle\Phi, \Phi\rangle\rangle=\alpha\langle\langle L(\Phi), \Phi\rangle\rangle=\alpha(\Phi, \Phi)_{g}=\alpha \geq \frac{1}{\lambda_{n+1}} .
$$

Also, for $Z_{0}=Z_{a}-\delta$ with $\delta>0$ small enough, we estimate from (3.38)

$$
\sum_{m=0}^{2 k} \int_{Z_{0}}^{Z_{a}}\left|\partial_{Z}^{m} \Phi(Z)\right|^{2} \frac{g}{\left(Z_{a}-Z\right)^{1-\nu}} Z^{d-1} d Z \leq c_{\nu} \delta^{\frac{\nu}{2}}\langle\langle\Phi, \Phi\rangle\rangle .
$$

On the other hand, from Rellich and an elementary compactness argument, for all $Z_{a}>0, \delta>0, \epsilon>0, k \geq 1$, there exists $c_{Z_{a}, \delta, \epsilon, k}>0$ such that

$$
\begin{aligned}
\sum_{m=0}^{2 k} \int_{Z \leq Z_{a}-\delta}\left|\partial_{Z}^{m} \Phi\right|^{2} Z^{d-1} d Z \leq & \epsilon \int_{Z \leq Z_{a}-\delta}\left|\partial_{Z} \Delta^{k} \Phi\right|^{2} Z^{d-1} d Z \\
& +c_{Z_{a}, \delta, \epsilon, k} \int_{Z \leq Z_{a}-\delta}|\Phi|^{2} Z^{d-1} d Z
\end{aligned}
$$

Summing the two inequalities yields for all $\delta>0$ small and $\epsilon$ smaller still:

$$
\begin{aligned}
& \sum_{m=0}^{2 k} \int_{0}^{Z_{a}}\left|\partial_{Z}^{m} \Phi(Z)\right|^{2} \frac{g}{\left(Z_{a}-Z\right)^{1-\nu}} Z^{d-1} d Z \\
& \quad \leq c_{\nu} \delta^{\frac{\nu}{2}}\langle\langle\Phi, \Phi\rangle\rangle+\tilde{c}_{Z_{a}, \delta, k} \int_{0}^{Z_{a}}|\Phi|^{2} g Z^{d-1} d Z .
\end{aligned}
$$


Together with (3.43), this implies for any $\Phi$ satisfying the orthogonality conditions $\left(\Phi, \Pi_{j, i}\right)_{g}=0, \quad 1 \leq i \leq I(j), \quad 1 \leq j \leq n$, and for any $\delta>0$

$\sum_{m=0}^{2 k} \int_{0}^{Z_{a}}\left|\partial_{Z}^{m} \Phi(Z)\right|^{2} \frac{g}{\left(Z_{a}-Z\right)^{1-\nu}} Z^{d-1} d Z \leq\left(c_{\nu} \delta^{\frac{\nu}{2}}+\tilde{c}_{\delta, Z_{a}, k} \lambda_{n+1}\right)\langle\langle\Phi, \Phi\rangle\rangle$,

which yields (3.37).

\subsection{Accretivity}

We now turn to the proof of the accretivity of the operator $m$.

Hilbert space. Recall (3.35). We define the space of test functions

$$
\mathscr{D}_{0}=\mathscr{D}_{\Phi} \times \mathscr{D}_{\Phi},
$$

and let $\mathbb{H}_{2 k}$ be the completion of $\mathscr{D}_{0}$ for the scalar product:

$$
\langle X, \tilde{X}\rangle=\langle\langle\Phi, \tilde{\Phi}\rangle\rangle+\left(T_{k}, \tilde{T}_{k}\right)_{g}+\int \chi T \overline{\tilde{T}} Z^{d-1} d Z
$$

which is a coercive Hermitian form from (3.36).

Unbounded operator. Following (3.15), we define the operator

$m=\left(\begin{array}{cc}-a H_{2} \Lambda & 1 \\ (p-1) Q \Delta-(1-a)^{2} H_{2}^{2} \Lambda^{2}+\tilde{A}_{2} \Lambda+A_{3} & -(2-a) H_{2} \Lambda+A_{2}\end{array}\right)$

with domain

$$
D(m)=\left\{X \in \mathbb{H}_{2 k}, \quad m X \in \mathbb{H}_{2 k}\right\}
$$

equipped with the domain norm. We then pick suitable directions $\left(X_{i}\right)_{1 \leq i \leq N} \in$ $\mathbb{H}_{2 k}$ and consider the finite rank projection operator

$$
\mathcal{A}=\sum_{i=1}^{N}\left\langle\cdot, X_{i}\right\rangle X_{i}
$$

The aim of this section is to prove the following accretivity property:

Proposition 3.10 (Maximal accretivity/dissipativity) Let

$$
\mu, \quad r>0 .
$$


There exist $k^{*} \gg 1$ and $0<c^{*}, a^{*} \ll 1$ such that for all $k \geq k^{*}, \forall 0<a<a^{*}$ small enough, there exist $N=N(k, a)$ directions $\left(X_{i}\right)_{1 \leq i \leq N} \in \mathbb{H}_{2 k}$ such that the modified unbounded operator

$$
\tilde{m}=m-\mathcal{A}
$$

is dissipative: ${ }^{7}$

$$
\forall X \in \mathscr{D}(m), \Re\langle-\tilde{m} X, X\rangle \geq c^{*} a k\langle X, X\rangle
$$

and maximal:

$\forall R>0, \quad \forall F \in \mathbb{H}_{2 k}, \quad \exists X \in \mathscr{D}(m)$ such that $(-\tilde{m}+R) X=F .(3.47)$

Remark 3.11 We recall that maximal dissipative operators are closed.

Proof of Proposition 3.10 given $R>R^{*}(k)$ large enough, we define the space of test functions

$$
\begin{aligned}
\mathscr{D}_{R}:= & \left\{X=(\Phi, T), \quad X \in C^{\frac{\sqrt{R}}{2}}\left(\left[0, Z_{a}\right]\right) \times C^{\frac{\sqrt{R}}{2}}\left(\left[0, Z_{a}\right]\right)\right\} \\
& \cap\left\{X /(-m+R) X \in C^{\infty}\left(\left[0, Z_{a}\right]\right) \times C^{\infty}\left(\left[0, Z_{a}\right]\right)\right\} .
\end{aligned}
$$

In steps 1-3 below, we prove (3.46) for $X \in \mathscr{D}_{R}$ so that all integrations by parts in steps $1-3$ are justified, and all boundary terms at $Z=Z_{a}$ vanish due to the vanishing of $g$ at $Z=Z_{a}$. In steps 4 and 5, for any smooth $F$ on $\left[0, Z_{a}\right]$, we show existence and uniqueness of a solution $X \in \mathbb{H}_{2 k}$ to $(-m+R) X=F$ for $R>R^{*}(k)$ large enough. In step 6, we prove that $\mathscr{D}_{R}$ is dense in $D(m)$. In step 7, we conclude the proof of (3.46) and (3.47).

Step 1 Main integration by parts. Let $X \in \mathscr{D}_{R}$ for $R>R^{*}(k)$ large enough. We aim at proving (3.46) and split the computation in two:

$$
\mid \begin{aligned}
& \langle X, \tilde{X}\rangle_{1}=-\left(\mathcal{L}_{g} \Phi_{k}, \tilde{\Phi}_{k}\right)_{g}+\left(T_{k}, \tilde{T}_{k}\right)_{g}, \\
& \langle X, \tilde{X}\rangle_{3}=\int \chi \Phi \tilde{\Phi}+\int \chi T \overline{\tilde{T}} .
\end{aligned}
$$

In step 1, we consider the principal part. We compute from (3.27):

$$
\begin{aligned}
& -\Re\langle m X, X\rangle_{1}=\Re\left(\mathcal{L}_{g} \Delta^{k}(m X)_{\Phi}, \Phi_{k}\right)_{g}-\Re\left(\Delta^{k}(m X)_{T}, T_{k}\right)_{g} \\
& =-\Re\left\{\int \nabla\left[-a H_{2} \Lambda \Phi_{k}-2 a k\left(H_{2}+\Lambda H_{2}\right) \Phi_{k}+T_{k}+\left(\widetilde{m_{k} X}\right)_{\Phi}\right]\right.
\end{aligned}
$$

\footnotetext{
7 Equivalently, $-\tilde{m}$ is accretive.
} 


$$
\begin{aligned}
& \left.-\overline{\nabla \Phi_{k}} Z^{2}\left(-D_{a}\right) Z^{d-1} \mu^{2} g d Z\right\} \\
& -\Re\left\{\int \left[\mathcal{L}_{g} \Phi_{k}-(2-a) H_{2} \Lambda T_{k}-2 k(2-a)\left(H_{2}+\Lambda H_{2}\right) T_{k}\right.\right. \\
& \left.\left.+A_{2} T_{k}+\left(\widetilde{m_{k}} X\right)_{T}\right] \overline{T_{k}} g Z^{d-1} d Z\right\} \\
= & -\Re\left\{\int \nabla \left[-a H_{2} \Lambda \Phi_{k}-2 a k\left(H_{2}+\Lambda H_{2}\right) \Phi_{k}\right.\right. \\
& \left.\left.+\left(\widetilde{m_{k}} X\right)_{\Phi}\right] \cdot \overline{\nabla \Phi_{k}} Z^{2}\left(-D_{a}\right) Z^{d-1} g \mu^{2} d Z\right\} \\
& -\Re\left\{\int \left[-(2-a) H_{2} \Lambda T_{k}-2 k(2-a)\left(H_{2}+\Lambda H_{2}\right) T_{k}\right.\right. \\
& \left.\left.+A_{2} T_{k}+\left(\widetilde{m_{k}} X\right)_{T}\right] \overline{T_{k}} g Z^{d-1} d Z\right\} .
\end{aligned}
$$

$T_{k}$ terms. We use

$$
\Re\left(\int f h \overline{\Lambda h}\right)=-\frac{1}{2} \int|h|^{2} f\left(d+\frac{\Lambda f}{f}\right)
$$

to compute

$$
\begin{aligned}
& -\Re\left\{\int\left[-(2-a) H_{2} \Lambda T_{k}\right] \overline{T_{k}} g Z^{d-1} d Z\right\} \\
& =-\frac{2-a}{2} \int\left|T_{k}\right|^{2} g H_{2}\left(d+\frac{\Lambda g}{g}+\frac{\Lambda H_{2}}{H_{2}}\right)
\end{aligned}
$$

and hence

$$
\begin{aligned}
& -\Re\left\{\int\left[-(2-a) H_{2} \Lambda T_{k}-2 k(2-a)\left(H_{2}+\Lambda H_{2}\right) T_{k}+A_{2} T_{k}\right] \overline{T_{k}} g Z^{d-1} d Z\right\} \\
& =(2-a) \int A_{5} H_{2}\left|T_{k}\right|^{2} g Z^{d-1} d Z
\end{aligned}
$$

with

$$
A_{5}:=-\frac{1}{2}\left[d+\frac{\Lambda g}{g}+\frac{\Lambda H_{2}}{H_{2}}\right]+2 k\left(1+\frac{\Lambda H_{2}}{H_{2}}\right)-\frac{A_{2}}{(2-a) H_{2}} .
$$

$\Phi_{k}$ terms. We first compute:

$$
\begin{aligned}
& -\Re\left\{\int \nabla\left[-2 a k\left(H_{2}+\Lambda H_{2}\right) \Phi_{k}\right] \cdot \overline{\nabla \Phi_{k}} Z^{2}\left(-D_{a}\right) Z^{d-1} g d Z\right\} \\
& =2 a k \int\left(H_{2}+\Lambda H_{2}\right)\left|\nabla \Phi_{k}\right|^{2} Z^{2}\left(-D_{a}\right) Z^{d-1} g d Z
\end{aligned}
$$




$$
\begin{aligned}
& +2 a k \Re\left\{\int \Phi_{k} \nabla\left(H_{2}+\Lambda H_{2}\right) \cdot \overline{\nabla \Phi_{k}} Z^{2}\left(-D_{a}\right) Z^{d-1} g d Z\right\} \\
= & 2 a k \int\left(H_{2}+\Lambda H_{2}\right)\left|\nabla \Phi_{k}\right|^{2} Z^{2}\left(-D_{a}\right) Z^{d-1} g d Z \\
& -a k \int\left|\Phi_{k}\right|^{2} \nabla \cdot\left(Z^{2}\left(-D_{a}\right) \nabla\left(H_{2}+\Lambda H_{2}\right) g\right) Z^{d-1} d Z .
\end{aligned}
$$

For the second term:

$$
\begin{aligned}
& -\Re\left\{\int \nabla\left[-a H_{2} \Lambda \Phi_{k}\right] \cdot \overline{\nabla \Phi_{k}} Z^{2}\left(-D_{a}\right) Z^{d-1} g d Z\right\} \\
& =-a \Re\left\{\int \partial_{Z}\left(H_{2} \Lambda \Phi_{k}\right) H_{2} \overline{\Lambda \Phi_{k}} \frac{D_{a} Z^{d}}{H_{2}} g d Z\right\} \\
& =\frac{a}{2} \int\left|H_{2} \Lambda \Phi_{k}\right|^{2} \frac{D_{a} Z^{d} g}{H_{2}}\left(\frac{\partial_{Z} D_{a}}{D_{a}}+\frac{d}{Z}-\frac{\partial_{Z} H_{2}}{H_{2}}+\frac{\partial_{Z} g}{g}\right) d Z \\
& =-\frac{a}{2} \int\left|\partial_{Z} \Phi_{k}\right|^{2} H_{2}\left(\frac{\Lambda D_{a}}{D_{a}}+d-\frac{\Lambda H_{2}}{H_{2}}+\frac{\Lambda g}{g}\right)\left(-D_{a}\right) g Z^{2} Z^{d-1} d Z .
\end{aligned}
$$

We have therefore obtained the formula:

$$
\begin{aligned}
& -\Re\langle m X, X\rangle_{1}=(2-a) \int A_{5} H_{2}\left|T_{k}\right|^{2} g+\mu^{2} a \\
& \times \int\left|\nabla \Phi_{k}\right|^{2} A_{6} Z^{2}\left(-D_{a}\right) Z^{d-1} g d Z \\
& -\mu^{2} a k \int\left|\Phi_{k}\right|^{2} \nabla \cdot\left(Z^{2}\left(-D_{a}\right) \nabla\left(H_{2}+\Lambda H_{2}\right) g\right) Z^{d-1} d Z \\
& -\mu^{2} \Re\left\{\int \nabla\left(\widetilde{m}_{k} X\right)_{\Phi} \cdot \overline{\nabla \Phi_{k}} Z^{2}\left(-D_{a}\right) Z^{d-1} g d Z\right\} \\
& -\Re\left\{\int\left(\widetilde{m_{k}} X\right)_{T} \overline{T_{k}} g Z^{d-1} d Z\right\}
\end{aligned}
$$

where we have defined

$$
A_{6}=2 k\left(H_{2}+\Lambda H_{2}\right)-\frac{H_{2}}{2}\left(\frac{\Lambda D_{a}}{D_{a}}+d-\frac{\Lambda H_{2}}{H_{2}}+\frac{\Lambda g}{g}\right) .
$$

We now claim the following lower bounds on $A_{5}, A_{6}$ : there exist universal constants $k^{*} \gg 1,0<c^{*}, a^{*} \ll 1$ such that for all $k \geq k^{*}$ and $0<a<a^{*}$,

$$
\forall 0 \leq Z \leq Z_{1}, \quad \mid \begin{aligned}
& A_{5} \geq \frac{c^{*} k}{Z_{a-} Z}, \\
& A_{6} \geq \frac{c^{*} k}{Z_{a}-Z}
\end{aligned} .
$$


Proof of (3.51). Recall (3.32), (3.33):

$$
\begin{aligned}
-\frac{\Lambda g}{g}= & \frac{1}{\left(-D_{a}\right)}\left\{-(d-1) \sigma^{2}+(1-w)^{2}-(d+1) D_{a}-\Lambda D_{a}\right. \\
& \left.+4 k\left[(1-a)^{2}(1-w)-\sigma F-(1-a)^{2}(1-w)(w+\Lambda w)\right]-\frac{\tilde{A}_{2}}{\mu^{2}}\right\} \\
= & \frac{4 k}{\left(-D_{a}\right)}\left[(1-w)-\sigma F-(1-w)(w+\Lambda w)+O\left(a+\frac{1}{k}\right)\right]
\end{aligned}
$$

and hence from (3.49):

$$
\begin{aligned}
& A_{5}=-\frac{1}{2}\left[d+\frac{\Lambda g}{g}+\frac{\Lambda H_{2}}{H_{2}}\right]+2 k\left(1+\frac{\Lambda H_{2}}{H_{2}}\right)-\frac{A_{2}}{(2-a) H_{2}} \\
&=\frac{2 k}{\left(-D_{a}\right)}\left[(1-w)-\sigma F-(1-w)(w+\Lambda w)+O\left(a+\frac{1}{k}\right)\right] \\
&+2 k\left(1-\frac{\Lambda w}{1-w}+O\left(\frac{1}{k}\right)\right) \\
&= \frac{2 k}{\left(-D_{a}\right)}[(1-w)-\sigma F-(1-w)(w+\Lambda w) \\
&\left.+\left(-D_{a}\right)\left(1-\frac{\Lambda w}{1-w}\right)+O\left(a+\frac{1}{k}\right)\right] \\
&= \frac{2 k}{\left(-D_{a}\right)}[(1-w)-\sigma F-(1-w)(w+\Lambda w) \\
&\left.+(-\Delta)\left(1-\frac{\Lambda w}{1-w}\right)+O\left(a+\frac{1}{k}\right)\right] .
\end{aligned}
$$

We now compute for $Z \leq Z_{2}$

$$
\begin{aligned}
& (1-w)-\sigma F-(1-w)(w+\Lambda w)+(-\Delta)\left(1-\frac{\Lambda w}{1-w}\right) \\
& =(1-w)(1-w-\Lambda w)-\sigma F+\left(\sigma^{2}-(1-w)^{2}\right) \frac{1-w-\Lambda w}{1-w} \\
& =\frac{\sigma^{2}(1-w-\Lambda w)}{1-w}-\sigma F=\frac{\sigma^{2}}{1-w}\left[1-w-\Lambda w-\frac{1-w}{\sigma} F\right] \\
& \geq c \sigma^{2}
\end{aligned}
$$


from the fundamental coercivity bound (2.21), and hence for $Z \leq Z_{a}$ and $a<a^{*}$ small enough:

$$
A_{5} \geq \frac{k c \sigma^{2}}{\left(-D_{a}\right)} \geq \frac{k c^{*}}{Z_{a}-Z}
$$

for some $c^{*}$ independent of $k, a$. Similarly:

$$
\begin{aligned}
A_{6}= & \frac{2 k H_{2}}{-D_{a}}\left\{\left[1+\frac{\Lambda H_{2}}{H_{2}}+O\left(\frac{1}{k}\right)\right]\right. \\
& \left.\quad\left(-D_{a}\right)+(1-w)-\sigma F-(1-w)(w+\Lambda w)+O\left(a+\frac{1}{k}\right)\right\} \\
= & \frac{2 k \mu(1-w)}{\left(-D_{a}\right)}[(1-w)-\sigma F-(1-w)(w+\Lambda w) \\
& \left.\quad+(-\Delta)\left(1-\frac{\Lambda w}{1-w}\right)+O\left(a+\frac{1}{k}\right)\right] \\
\geq & \frac{k c^{*}}{Z_{a}-Z}
\end{aligned}
$$

arguing as above. This concludes the proof of (3.51).

Step 2 No derivatives term. We compute

$$
\begin{aligned}
-\Re\langle m X, X\rangle_{3}= & -\Re\left\{\int \chi(m X)_{\Phi} \bar{\Phi} Z^{d-1} d Z\right. \\
& \left.+\int \chi(m X)_{T} \bar{T} Z^{d-1} d Z\right\} \\
= & -\Re\left\{\int \chi\left[-a H_{2} \Lambda \Phi+T\right] \bar{\Phi} Z^{d-1} d Z\right\} \\
& -\Re\left\{\int \chi \left[(p-1) Q \Delta \Phi-(1-a)^{2} H_{2}^{2} \Lambda^{2} \Phi+\tilde{A}_{2} \Lambda \Phi\right.\right. \\
& \left.\left.+A_{3} \Phi-(2-a) H_{2} \Lambda T+A_{2} T\right] \bar{T}\right\} \\
= & O\left(\int ( \chi + | \Lambda \chi | ) \left(|\Phi|^{2}+\left|\partial_{Z} \Phi\right|^{2}\right.\right. \\
& \left.\left.+\left|\partial_{Z}^{2} \Phi\right|^{2}+|T|^{2}\right)\right) .
\end{aligned}
$$


Step 3 Accretivity in $\mathscr{D}_{0}$. We compute from (3.52), (3.50):

$$
\begin{aligned}
& -\Re\langle m X, X\rangle=-\Re\langle m X, X\rangle_{1}-\Re\langle m X, X\rangle_{3} \\
& =(2-a) \int A_{5} H_{2}\left|T_{k}\right|^{2} g+\mu^{2} a \int\left|\nabla \Phi_{k}\right|^{2} A_{6} Z^{2}\left(-D_{a}\right) Z^{d-1} g d Z \\
& -\mu^{2} a k \int\left|\Phi_{k}\right|^{2} \nabla \cdot\left(Z^{2}\left(-D_{a}\right) \nabla\left(H_{2}+\Lambda H_{2}\right) g\right) Z^{d-1} d Z \\
& -\mu^{2} \Re\left\{\int \nabla\left(\widetilde{m_{k}} X\right)_{\Phi} \cdot \overline{\nabla \Phi_{k}} Z^{2}\left(-D_{a}\right) Z^{d-1} g d Z\right\} \\
& -\Re\left\{\int\left(\widetilde{m_{k}} X\right)_{T} \overline{T_{k}} g Z^{d-1} d Z\right\} \\
& +O\left(\int(\chi+|\Lambda \chi|)\left(|\Phi|^{2}+\left|\partial_{Z} \Phi\right|^{2}+\left|\partial_{Z}^{2} \Phi\right|^{2}+|T|^{2}\right)\right) .
\end{aligned}
$$

We lower bound from (3.51) and the fact that $H_{2} \gtrsim 1$ :

$$
\begin{aligned}
& (2-a) \int A_{5} H_{2}\left|T_{k}\right|^{2} g+\mu^{2} a \int\left|\nabla \Phi_{k}\right|^{2} A_{6} Z^{2}\left(-D_{a}\right) Z^{d-1} g d Z \\
& \geq c^{*} a k\left[\int\left(\frac{\left|T_{k}\right|^{2}}{Z_{a}-Z}+\left|\nabla \Phi_{k}\right|^{2} \frac{Z^{2}\left(-D_{a}\right)}{Z_{a}-Z}\right) g Z^{d-1} d Z\right] .
\end{aligned}
$$

The smoothness and boundedness of the profile together with (3.32), (3.33) ensure that

$$
\left|\nabla \cdot\left[Z^{2}\left(-D_{a}\right) \nabla\left(H_{2}+\Lambda H_{2}\right) g\right]\right| \leq C_{k} \frac{Z^{2}\left(-D_{a}\right) g}{Z_{a}-Z} \leq C_{k} g
$$

and in view of (3.28),

$$
\begin{aligned}
& \mid-\Re\left\{\int \nabla\left(\widetilde{m_{k}} X\right)_{\Phi} \cdot \overline{\nabla \Phi_{k}} Z^{2}\left(-D_{a}\right) Z^{d-1} g d Z\right\} \\
& -\Re\left\{\int\left(\widetilde{m_{k}} X\right)_{T} \overline{T_{k}} g Z^{d-1} d Z\right\} \\
& \leq C_{k}\left(\int\left|\nabla \Phi_{k}\right|^{2} Z^{2}\left(-D_{a}\right) g Z^{d-1} d Z\right)^{\frac{1}{2}}\left(\sum_{j=0}^{2 k} \int\left|\partial_{Z}^{j} \Phi\right|^{2} g Z^{d-1} d Z\right)^{\frac{1}{2}}
\end{aligned}
$$




$$
\begin{aligned}
& +C_{k}\left(\int\left|T_{k}\right|^{2} g Z^{d-1} d Z\right)^{\frac{1}{2}}\left[\left(\sum_{j=0}^{2 k-1} \int\left|\partial_{Z}^{j} T\right|^{2} g Z^{d-1} d Z\right)^{\frac{1}{2}}\right. \\
& \left.+\left(\sum_{j=0}^{2 k} \int\left|\partial_{Z}^{j} \Phi\right|^{2} g Z^{d-1} d Z\right)^{\frac{1}{2}}\right]
\end{aligned}
$$

The collection of above bounds yields:

$$
\begin{aligned}
-\Re\langle m X, X\rangle \geq & c^{*} a k\left[\int \frac{\left|T_{k}\right|^{2}}{Z_{a}-Z} g Z^{d-1} d Z\right. \\
& \left.+\int\left|\nabla \Phi_{k}\right|^{2} \frac{Z^{2}\left(-D_{a}\right)}{Z_{a}-Z} g Z^{d-1} d Z\right] \\
& -C_{k}\left[\sum_{j=0}^{2 k} \int\left|\partial_{Z}^{j} \Phi\right|^{2} g Z^{d-1} d Z+\sum_{j=0}^{2 k-1} \int\left|\partial_{Z}^{j} T\right|^{2} g Z^{d-1} d Z\right] .
\end{aligned}
$$

We conclude using (3.37) with $N=N(a, k)$ large enough and its analogue for $T$ :

$$
-\Re\langle m X, X\rangle \geq c^{*} a k\langle X, X\rangle-C_{a, k} \sum_{i=1}^{N}\left(\left(\Phi, \Pi_{i}\right)_{g}^{2}+\left(T, \mathcal{T}_{i}\right)_{g}^{2}\right) .
$$

Therefore,

$$
\begin{aligned}
-\Re\langle(m-\mathcal{A}) X, X\rangle \geq & c^{*} a k\langle X, X\rangle+\sum_{i=1}^{N}\left(\left\langle X, X_{i, 1}\right\rangle^{2}+\left\langle X, X_{i, 2}\right\rangle^{2}\right. \\
& \left.-C_{a, k}\left[\left(\Phi, \Pi_{i}\right)_{g}^{2}+\left(T, \mathcal{T}_{i}\right)_{g}^{2}\right]\right) .
\end{aligned}
$$

The linear from

$$
X=(\Phi, T) \mapsto \sqrt{C_{a, k}}\left(\Phi, \Pi_{i}\right)_{g}
$$

from $\left(\mathbb{H}_{2 k},\langle\cdot\rangle\right)$ into $\mathbb{C}$ is continuous from Cauchy-Schwarz and (3.36), and hence by Riesz theorem, there exists $X_{i} \in \mathbb{H}_{2 k}$ such that

$$
\forall X \in \mathbb{H}_{2 k}, \quad\left\langle X, X_{i}\right\rangle=\left(\Phi, \Pi_{i}\right)_{g},
$$

and similarly for $\mathcal{T}_{i}$, and (3.46) follows for $X \in \mathscr{D}_{R}$. 
Step 4 ODE formulation of maximality. Our goal, in steps 4-6, is to prove that for all $R>0$ large enough,

$$
\forall F \in C^{\infty}\left(\left[0, Z_{a}\right]\right), \quad \exists ! X \in \mathbb{H}_{2 k} \quad \text { such that } \quad(-m+R) X=F .(3.53)
$$

(3.53) corresponds to solving

$$
\mid \begin{aligned}
& -\left[-a H_{2} \Lambda\right] \Phi-T+R \Phi=F_{\Phi}, \\
& -\left\{\left[(p-1) Q \Delta-(1-a)^{2} H_{2}^{2} \Lambda^{2}+\tilde{A}_{2} \Lambda+A_{3}\right] \Phi\right. \\
& \left.\quad-(2-a) H_{2} \Lambda T+A_{2} T\right\}+R T=F_{T} .
\end{aligned}
$$

Solving for $T$ :

$$
T=\left(a H_{2} \Lambda+R\right) \Phi-F_{\Phi},
$$

we look for $\Phi$ —solution to the second order elliptic equation:

$$
\begin{aligned}
& {\left[(p-1) Q \Delta-(1-a)^{2} H_{2}^{2} \Lambda^{2}+\tilde{A}_{2} \Lambda+A_{3}\right] \Phi} \\
& \quad\left[-(2-a) H_{2} \Lambda+A_{2}\right]\left[a H_{2} \Lambda \Phi+R \Phi-F_{\Phi}\right] \\
& \quad=-F_{T}+R\left(a H_{2} \Lambda \Phi+R \Phi-F_{\Phi}\right)
\end{aligned}
$$

i.e.

$$
\begin{aligned}
(p-1) Q \Delta \Phi-H_{2}^{2} \Lambda^{2} \Phi+\Lambda \Phi\left[\tilde{A}_{2}+a H_{2} A_{2}-2 R H_{2}\right. \\
\left.\quad-a(2-a) H_{2} \Lambda H_{2}\right]+\left(A_{3}+R A_{2}-R^{2}\right) \Phi \\
=-F_{T}-R F_{\Phi}+\left[-(2-a) H_{2} \Lambda+A_{2}\right] F_{\Phi} .
\end{aligned}
$$

Now, we have

$$
\begin{aligned}
(p-1) Q \Delta \Phi-H_{2}^{2} \Lambda^{2} \Phi= & \left((p-1) Q-H_{2}^{2} Z^{2}\right) \partial_{Z}^{2} \Phi \\
& +\left(\frac{(d-1)(p-1) Q}{Z}-H_{2}^{2} Z\right) \partial_{Z} \Phi
\end{aligned}
$$

and hence

$$
\begin{aligned}
& \left((p-1) Q-H_{2}^{2} Z^{2}\right) \partial_{Z}^{2} \Phi+\left\{\frac{(d-1)(p-1) Q}{Z}-H_{2}^{2} Z\right. \\
& \left.+Z\left[\tilde{A}_{2}+a H_{2} A_{2}-2 R H_{2}-a(2-a) H_{2} \Lambda H_{2}\right]\right\} \partial_{Z} \Phi
\end{aligned}
$$




$$
\begin{aligned}
& +\left(A_{3}+R A_{2}-R^{2}\right) \Phi \\
= & -F_{T}-R F_{\Phi}+\left[-(2-a) H_{2} \Lambda+A_{2}\right] F_{\Phi} .
\end{aligned}
$$

Since $(p-1) Q=\mu^{2} Z^{2} \sigma^{2}$, we have

$$
\begin{aligned}
& \left((p-1) Q-H_{2}^{2} Z^{2}\right) \partial_{Z}^{2} \Phi+\left\{\frac{(d-1)(p-1) Q}{Z}-H_{2}^{2} Z\right. \\
& \left.\quad+Z\left[\tilde{A}_{2}+a H_{2} A_{2}-2 R H_{2}-a(2-a) H_{2} \Lambda H_{2}\right]\right\} \partial_{Z} \Phi \\
& =\left(\mu^{2} \sigma^{2}-H_{2}^{2}\right) Z^{2} \partial_{Z}^{2} \Phi+\left\{(d-1) \mu^{2} Z \sigma^{2}-H_{2}^{2} Z\right. \\
& \left.\quad+Z\left[\tilde{A}_{2}+a H_{2} A_{2}-2 R H_{2}-a(2-a) H_{2} \Lambda H_{2}\right]\right\} \partial_{Z} \Phi \\
& =\frac{1}{Z^{d-1} \varpi} \partial_{Z}\left(Z^{d-1} \varpi\left(\mu^{2} \sigma^{2}-H_{2}^{2}\right) Z^{2} \partial_{Z} \Phi\right)
\end{aligned}
$$

with

$$
\begin{aligned}
& \left(\frac{\partial_{Z} \varpi}{\varpi}+\frac{d-1}{Z}\right)\left(\mu^{2} \sigma^{2}-H_{2}^{2}\right) Z^{2} \\
& \quad+2 Z\left(\mu^{2} \sigma^{2}-H_{2}^{2}\right)+\left(2 \mu^{2} \sigma \partial_{Z} \sigma-2 H_{2} \partial_{Z} H_{2}\right) Z^{2} \\
& =(d-1) \mu^{2} Z \sigma^{2}-H_{2}^{2} Z \\
& \quad+Z\left[\tilde{A}_{2}+a H_{2} A_{2}-2 R H_{2}-a(2-a) H_{2} \Lambda H_{2}\right]
\end{aligned}
$$

i.e.

$$
\begin{aligned}
\frac{\partial_{Z} \varpi}{\varpi}= & -\frac{2}{Z}-\frac{2 \mu^{2} \sigma \partial_{Z} \sigma-2 H_{2} \partial_{Z} H_{2}}{\mu^{2} \sigma^{2}-H_{2}^{2}} \\
& -\frac{2 R H_{2}-(d-2) H_{2}^{2}-\tilde{A}_{2}-a H_{2} A_{2}+a(2-a) H_{2} \Lambda H_{2}}{\left(\mu^{2} \sigma^{2}-H_{2}^{2}\right) Z} .
\end{aligned}
$$

Recalling $H_{2}=\mu(1-w)$ yields

$$
\begin{aligned}
\frac{\partial_{Z} \varpi}{\omega}=- & \frac{2}{Z}-\frac{\partial_{Z}\left[\sigma^{2}-(1-w)^{2}\right]}{\sigma^{2}-(1-w)^{2}} \\
& -\frac{\frac{\frac{2(1-w)}{\mu} R-(d-2)(1-w)^{2}-\frac{\tilde{A}_{2}}{\mu^{2}}-a(1-w) \frac{A_{2}}{\mu}-a(2-a)(1-w) \Lambda w}{Z\left(\sigma^{2}-(1-w)^{2}\right)} .}{} .
\end{aligned}
$$


We therefore define

$$
\varpi(Z)=\mid \begin{array}{ll}
\frac{e^{-F_{-}(Z)}}{Z^{2}\left(\sigma^{2}-(1-w)^{2}\right)} & \text { for } 0 \leq Z \leq Z_{2}, \\
\frac{e^{-F_{+}(Z)}}{Z^{2}\left(\sigma^{2}-(1-w)^{2}\right)} & \text { for } Z>Z_{2} .
\end{array}
$$

where $^{8}$

$$
\begin{aligned}
& F_{-}(Z)=\int_{\frac{Z_{2}}{2}}^{Z} \frac{\frac{2(1-w)}{\mu} R-(d-2)(1-w)^{2}-\frac{\tilde{A}_{2}}{\mu^{2}}-a(1-w) \frac{A_{2}}{\mu}-a(2-a)(1-w) \Lambda w}{Z^{\prime}\left(\sigma^{2}-(1-w)^{2}\right)} d Z^{\prime}+C_{-}, \\
& F_{+}(Z)=\int_{2 Z_{2}}^{Z} \frac{\frac{2(1-w)}{\mu} R-(d-2)(1-w)^{2}-\frac{\tilde{A}_{2}}{\mu^{2}}-a(1-w) \frac{A_{2}}{\mu}-a(2-a)(1-w) \Lambda w}{Z^{\prime}\left(\sigma^{2}-(1-w)^{2}\right)} d Z^{\prime}+C_{+} .
\end{aligned}
$$

In view of the above, we have obtained the elliptic equation:

$$
\mid \begin{aligned}
& -\frac{1}{Z^{d-1} \varpi} \partial_{Z}\left(Z^{d-1} \varpi\left(\sigma^{2}-(1-w)^{2}\right) Z^{2} \partial_{Z} \Phi\right) \\
& \quad+\frac{1}{\mu^{2}}\left(R^{2}-A_{2} R-A_{3}\right) \Phi=H, \\
& H=\frac{1}{\mu^{2}}\left\{F_{T}+R F_{\Phi}+\left[(2-a) H_{2} \Lambda-A_{2}\right] F_{\Phi}\right\}
\end{aligned},
$$

with $T$ recovered by (3.54). As $Z \rightarrow Z_{2}$, we have from (3.24):

$$
\Delta(Z)=\frac{\left|\lambda_{+}\right|}{Z_{2}}\left(Z-Z_{2}\right)+O\left(\left(Z-Z_{2}\right)^{2}\right)
$$

and hence

$$
Z\left(\sigma^{2}-(1-w)^{2}\right)=\left|\lambda_{+}\right|\left(Z_{2}-Z\right)\left[1+O\left(Z-Z_{2}\right)\right]
$$

and hence

$$
\begin{aligned}
& \frac{\frac{2(1-w)}{\mu} R-(d-2)(1-w)^{2}-\frac{\tilde{A}_{2}}{\mu^{2}}-a(1-w) \frac{A_{2}}{\mu}-a(2-a)(1-w) \Lambda w}{Z\left(\sigma^{2}-(1-w)^{2}\right)} \\
& =\frac{\frac{2 \sigma_{2}}{\mu\left|\lambda_{+}\right|} R\left[1+O\left(\frac{1}{R}\right)\right]}{\left(Z_{2}-Z\right)\left[1+O\left(Z-Z_{2}\right)\right]} .
\end{aligned}
$$

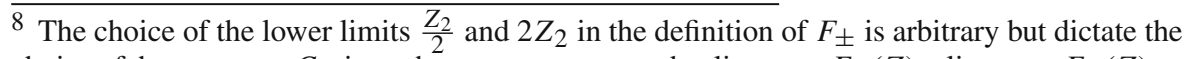
choice of the constants $C_{ \pm}$in such a way as to ensure that $\lim _{Z \uparrow Z_{2}} F_{-}(Z)-\lim _{Z \downarrow Z_{2}} F_{+}(Z)=$ 0 . The additional degree of freedom in the choice of $C_{ \pm}$is used to fix an overall normalization of $\varpi$. 
Since the profile passes through $P 2$ in a $C^{\infty}$ way, we obtain the development of the measure at $P 2$ : for any $M \geq 1$,

$\varpi(Z)=\left|Z_{2}-Z\right|^{c_{\varpi}}\left[1+\sum_{m=0}^{M} d_{\sigma, m, R}\left(Z_{2}-Z\right)^{m}+O_{M}\left(\left|Z_{2}-Z\right|^{M+1}\right)\right]$,

where

$$
c_{\varpi}=\frac{2 \sigma_{2}}{\mu\left|\lambda_{+}\right|} R\left[1+O\left(\frac{1}{R}\right)\right] \geq c^{*} R>0
$$

for $R>R^{*}$ large enough. Note that the above choice of $C_{ \pm}$is made to fix the normalization constant in front of $\left|Z_{2}-Z\right|^{c_{\varpi}}$ to be equal to 1 .

Step 5 Solving (3.56). We analyze the singularity of (3.56) at $P 2$ using a change of variables.

$0 \leq Z<Z_{2}$. We let

$$
\Phi(Z)=\Psi(Y), \quad Y=h(Z), \quad h(Z)=\int_{\frac{z_{2}}{2}}^{Z} \frac{d Z^{\prime}}{Z^{\prime d-1} \varpi Z^{\prime 2}\left(\sigma^{2}-(1-w)^{2}\right)},
$$

which maps (3.56) onto:

$$
\mid \begin{aligned}
& -\partial_{Y}^{2} \Psi+\frac{1}{\mu^{2}}\left(R^{2}-A_{2} R-A_{3}\right) Z^{2 d} \varpi^{2}\left(\sigma^{2}-(1-w)^{2}\right) \Psi=\tilde{H}, \\
& \tilde{H}=Z^{2 d} \varpi^{2}\left(\sigma^{2}-(1-w)^{2}\right) H .
\end{aligned}
$$

From (3.57),

$$
\begin{aligned}
Y & =h(Z)=\int_{\frac{Z_{2}}{2}}^{Z} \frac{d z}{z^{d-1} \varpi z^{2}\left(\sigma^{2}-(1-w)^{2}\right)} \\
& =\int_{\frac{Z_{2}}{2}}^{Z} \frac{d z}{z^{d-1} z\left|\lambda_{+}\right|\left(Z_{2}-z\right)\left(Z_{2}-z\right)^{c_{\sigma}}\left[1+\sum_{m=0}^{M} d_{\sigma, m, R}\left(Z_{2}-z\right)^{m}+O_{M}\left(\left|Z_{2}-z\right|^{M+1}\right)\right]} \\
& =\frac{C}{R \varpi}[1+\Gamma(Z)],
\end{aligned}
$$

where from (3.58) constant $C>0$ is independent of $R$ and, choosing $M=$ $\sqrt{R}$

$$
\Gamma(Z)=\sum_{m=1}^{\sqrt{R}} \tilde{d}_{\sigma, m, R}\left(Z_{2}-Z\right)^{m}+O\left(\left(Z_{2}-Z\right)^{\sqrt{R}+1}\right)
$$


with similar estimates for derivatives. Hence the potential term in (3.59) can be expanded in $Y$ and estimated as $Y \rightarrow+\infty$ for $R$ large enough:

$$
\begin{aligned}
& \frac{1}{\mu^{2}}\left(R^{2}-A_{2} R-A_{3}\right) Z^{2 d} \varpi^{2}\left(\sigma^{2}-(1-w)^{2}\right) \\
& \quad=\frac{C_{R}}{Y^{2+c_{R}}}\left[1+\sum_{j=1}^{\sqrt{R}} \frac{\tilde{\tilde{d}}_{j}}{Y^{j c_{R}}}+O\left(\frac{1}{Y^{c_{R}(\sqrt{R}+1)}}\right)\right]
\end{aligned}
$$

for some universal constants $\tilde{\tilde{d}}_{j}$,

$$
C_{R}=C+O\left(\frac{1}{R}\right), \quad 0<c_{R}=\frac{1}{c_{\varpi}} \lesssim \frac{1}{R}
$$

where $C>0$ is independent of R. Therefore, by an elementary fixed point argument, (3.59) with $\tilde{H}=0$ admits a basis of solutions $\Psi_{1}^{-}$and $\Psi_{2}^{-}$with the following behavior as $Y \rightarrow+\infty$

$$
\mid \begin{aligned}
& \Psi_{1}^{-}=1+\sum_{j=1}^{\sqrt{R}} \frac{c_{j, 1}}{Y^{j c_{R}}}+O\left(\frac{1}{Y^{(\sqrt{R}+1) c_{R}}}\right) \\
& \Psi_{2}^{-}=Y\left[1+\sum_{j=1}^{\sqrt{R}} \frac{c_{j, 2}}{Y^{j c_{R}}}+O\left(\frac{1}{Y^{(\sqrt{R}+1) c_{R}}}\right)\right]
\end{aligned}
$$

with similar estimates for derivatives. The sequences $\left(c_{j, 1}\right)_{j=1,2}$ are uniquely determined inductively from (3.59) with $\tilde{H}=0$ using the expansion of the potential (3.62).

$Z_{2}<Z \leq Z_{a}$. To the right of $P 2$, we let

$\Phi(Z)=\Psi(Y), \quad Y=h(Z), \quad h(Z)=\int_{2 Z_{2}}^{Z} \frac{d z}{z^{d-1} \varpi z^{2}\left(\sigma^{2}-(1-w)^{2}\right)}+\tilde{C}_{+}$,

which sends ${ }^{9} Y \rightarrow+\infty$ as $Z \downarrow Z_{2}$. We construct a similar basis of homogenous solutions $\Psi_{1}^{+}$and $\Psi_{2}^{+}$as $Y \rightarrow+\infty$ with asymptotics given by

$$
\begin{aligned}
& \Psi_{1}^{+}=1+\sum_{j=1}^{\sqrt{R}} \frac{c_{j, 1}}{Y^{j c_{R}}}+O\left(\frac{1}{Y^{(1+\sqrt{R}) c_{R}}}\right), \\
& \Psi_{2}^{+}=Y\left[1+\sum_{j=1}^{\sqrt{R}} \frac{c_{j, 2}}{Y^{j c_{R}}}+O\left(\frac{1}{Y^{(1+\sqrt{R}) c_{R}}}\right)\right]
\end{aligned}
$$

\footnotetext{
$\overline{9}$ We add constant $\tilde{C}_{+}$to match the asymptotic expansion of $Y$ in terms of $\left(Z_{2}-Z\right)$. In principle, it is unnecessary as it influences the terms of order $R$ and higher while we only need the universality of the expansion up to the order $\sqrt{R}$.
} 
with the sequences $c_{j, 1}, c_{j, 2}$ the same as in (3.63).

Basis of fundamental solutions. The function $\Phi_{1}(Z)=\Psi_{1}^{-}(Y)$ for $Z<Z_{2}$ and $\Phi_{1}(Z)=\Psi_{1}^{+}(Y)$ for $Z>Z_{2}$, obtained by gluing $\Psi_{1}^{ \pm}(Y)$ belongs to $e^{\sqrt{R}}\left(\left[0, Z_{a}\right]\right)$ and is a solution to the homogeneous Eq. (3.59). Let now $\Phi_{\mathrm{rad}}(Z)$ be the radial solution to the homogeneous problem associated to (3.56) with $\Phi_{\mathrm{rad}}(0)=1$. Then the wronskian is given by

$$
W=\partial_{Z} \Phi_{1} \Phi_{\mathrm{rad}}-\partial_{Z} \Phi_{\mathrm{rad}} \Phi_{1}=\frac{W_{0}}{Z^{d-1} \varpi Z^{2}\left(\sigma^{2}-(1-w)^{2}\right)}
$$

where $W_{0}$ is a constant. We claim $W_{0} \neq 0$. Indeed, otherwise $\Phi_{\text {rad }}$ is proportionate to $\Phi_{1}$ and hence is $C^{\sqrt{R}}$ on $\left[0, Z_{a}\right]$. In particular, if $T_{\text {rad }}$ is given by (3.54) with $F_{\Phi}=0$, then $X_{\text {rad }}=\left(\Phi_{\text {rad }}, T_{\text {rad }}\right)$ satisfies

$$
(-m+R) X_{\text {rad }}=0 \text { on }\left(0, Z_{a}\right) .
$$

Since $X_{\text {rad }}$ is $C^{\sqrt{R}-1}\left[\left[0, Z_{2}\right]\right)$, we may apply the analysis in steps $1-4$ for $R>R^{*}(k)$ large enough and (3.46) holds for $X_{\text {rad }}$, i.e.

$$
\begin{aligned}
0 & =\Re\left\langle(-m+R) X_{\text {rad }}, X_{\text {rad }}\right\rangle \\
& =\Re\left\langle(-m+\mathcal{A}) X_{\text {rad }}, X_{\text {rad }}\right\rangle-\Re\left\langle\mathcal{A} X_{\text {rad }}, X_{\text {rad }}\right\rangle+R\left\|X_{\text {rad }}\right\|_{\mathbb{H}_{2 k}}^{2} \\
& \geq R\left\|X_{\text {rad }}\right\|_{\mathbb{H}_{2 k}}^{2}-\left\langle\mathcal{A} X_{\text {rad }}, X_{\text {rad }}\right\rangle
\end{aligned}
$$

so that for $R>R^{*}(k)$ sufficiently large

$$
\frac{R}{2}\left\|X_{\text {rad }}\right\|_{\mathbb{H}_{2 k}}^{2} \leq 0
$$

and hence $X_{r a d}=0$ a contradiction. This concludes the proof of $W_{0} \neq 0$.

Inner solution of the inhomogeneous problem. $\left(\Phi_{\mathrm{rad}}, \Phi_{1}\right)$ is then a basis for the homogeneous problem corresponding to (3.56). As a consequence, the only solution to (3.56) which is $o\left(\left(Z_{2}-Z\right)^{-\frac{1}{c_{R}}}\right)$ at $Z=Z_{2}$ is given by ${ }^{10}$

$$
\Phi(Z)=-\Phi_{1}(Z) \int_{0}^{Z} \frac{H(\tau) \Phi_{\mathrm{rad}}(\tau)}{W(\tau)} d \tau-\Phi_{\mathrm{rad}}(Z) \int_{Z}^{Z_{2}} \frac{H(\tau) \Phi_{1}(\tau)}{W(\tau)} d \tau .
$$

$\overline{10}$ Note that $\Phi_{\text {rad }}(Z) \sim\left(Z_{2}-Z\right)^{-\frac{1}{c_{R}}}$ as $Z \rightarrow Z_{2}$ in view of the behavior of $\Psi_{2}$ as $Y \rightarrow+\infty$. 
For a smooth $H, \Phi$ is smooth on $\left[0, Z_{2}\right)$ and we study its regularity at $Z_{2}$. In $Y$ variables we obtain for some $Y_{0}$ large enough:

$$
\begin{aligned}
\Psi(Y)= & c_{Y_{0}, H} \Psi_{1}^{-}(Y)-\Psi_{1}^{-}(Y) \int_{Y_{0}}^{Y} \tilde{H}(\tau) \Psi_{2}^{-}(\tau) d \tau \\
& -\Psi_{2}^{-}(Y) \int_{Y}^{+\infty} \tilde{H}(\tau) \Psi_{1}^{-}(\tau) d \tau .
\end{aligned}
$$

We have from (3.57), (3.60):

$$
(R Y)^{c_{R}}=\frac{1}{Z_{2}-Z}\left(\sum_{m=0}^{\sqrt{R}} \beta_{m}\left(Z_{2}-Z\right)^{m}+O\left(\left|Z_{2}-Z\right|^{\sqrt{R}+1}\right)\right),
$$

and hence

$$
Z_{2}-Z=\sum_{m=1}^{\sqrt{R}} \frac{y_{m}}{Y^{m c_{R}}}+O\left(\frac{1}{Y^{(\sqrt{R}+1) c_{R}}}\right)
$$

with similar estimates for derivatives. In particular, a smooth function $H(Z)$ yields expansion for $\tilde{H}(Z)$ :

$$
\begin{aligned}
\tilde{H} & =\left(Z_{2}-Z\right)^{1+2 c_{R}^{-1}}\left(\sum_{m=0}^{\sqrt{R}} h_{m}\left(Z_{2}-Z\right)^{m}+O\left(\left(Z_{2}-Z\right)^{\sqrt{R}+1}\right)\right) \\
& =\sum_{m=1}^{\sqrt{R}} \frac{q_{m}}{Y^{2+m c_{R}}}+O\left(\frac{1}{Y^{2+(\sqrt{R}+1)} c_{R}}\right) .
\end{aligned}
$$

Conversely, an expansion of the form

$$
G=\sum_{m=0}^{\sqrt{R}-1} \frac{b_{m}}{Y^{m c_{R}}}+O\left(\frac{1}{Y^{\sqrt{R} c_{R}}}\right)
$$

defines a $C^{\sqrt{R}}$ function $G(Z)$ at $Z=Z_{2}$. Plugging in the asymptotic expansion for $\Psi_{1}^{-}, \Psi_{2}^{-}$and $\tilde{H}$ in (3.64) yields 


$$
\begin{aligned}
& \Psi(Y)=c_{Y_{0}, H}\left(\sum_{m=0}^{\sqrt{R}} \frac{c_{m, 1}}{Y^{m c_{R}}}+O\left(\frac{1}{Y^{(\sqrt{R}+1) c_{R}}}\right)\right) \\
& -\left(\sum_{m=0}^{\sqrt{R}} \frac{c_{m, 1}}{Y^{m c_{R}}}+O\left(\frac{1}{Y^{(\sqrt{R}+1) c_{R}}}\right)\right) \\
& \times \int_{Y_{0}}^{Y}\left(\sum_{m=0}^{\sqrt{R}} \frac{c_{m, 2}}{\tau^{m c_{R}}}+O\left(\frac{1}{Y^{(\sqrt{R}+1) c_{R}}}\right)\right) \\
& \times\left(\sum_{j=1}^{\sqrt{R}} \frac{q_{j}}{\tau^{1+j c_{R}}}+O\left(\frac{1}{\tau^{1+(\sqrt{R}+1) c_{R}}}\right)\right) d \tau \\
& -\left(\sum_{m=0}^{\sqrt{R}} \frac{c_{m, 2}}{Y^{m c_{R}}} Y+O\left(\frac{1}{Y^{(\sqrt{R}+1) c_{R}}}\right)\right) \\
& \int_{Y}^{\infty}\left(\sum_{m=0}^{\sqrt{R}} \frac{c_{m, 1}}{\tau^{m c_{R}}}+O\left(\frac{\log (\tau)}{\tau^{(\sqrt{R}+1) c_{R}}}\right)\right) \\
& \times\left(\sum_{j=1}^{\sqrt{R}} \frac{q_{j}}{\tau^{2+j c_{R}}}+O\left(\frac{1}{\tau^{2+(\sqrt{R}+1) c_{R}}}\right)\right) d \tau \\
& =\sum_{m=0}^{\sqrt{R}-1} \frac{b_{m}}{Y^{m c_{R}}}+O\left(\frac{1}{Y^{\sqrt{R} c_{R}}}\right) \text {. }
\end{aligned}
$$

We therefore have proved that for $H \in C^{\infty}\left(\left[0, Z_{2}\right]\right)$, there exists a unique solution $\Phi$ to (3.56) on $\left[0, Z_{2}\right]$ which is $o\left(\left(Z_{2}-Z\right)^{-\frac{1}{c_{R}}}\right)$ at $Z=Z_{2}$. Furthermore, this solution is smooth on $\left[0, Z_{2}\right)$, and is $C^{\sqrt{R}}$ at $Z=Z_{2}$ where it admits an asymptotic expansion

$$
\Phi(Z)=\sum_{j=0}^{\sqrt{R}-1} c_{j, \Phi}\left(Z_{2}-Z\right)^{j}+O\left(\left(Z_{2}-Z\right)^{\sqrt{R}}\right) .
$$

Outer solution of the inhomogeneous problem. We argue similarly, considering the basis $\Phi_{1}(Z)$ and $\Phi_{\text {rad }}^{+}(Z)$ with $\Phi_{\text {rad }}^{+}\left(Z_{a}\right)=1$, for $Z_{2}<Z \leq Z_{a}$ and construct $\Phi$ solution to (3.56) on $\left[Z_{2}, Z_{a}\right]$ which is smooth on $\left(Z_{2}, Z_{a}\right]$, $o\left(\left(Z_{2}-Z\right)^{-\frac{1}{c_{R}}}\right)$ at $Z=Z_{2}$ and $C^{\sqrt{R}}$ at $Z=Z_{2}$. Furthermore, $\Phi$ admits at 
$Z=Z_{2}$ the following asymptotic expansion analogous to (3.65)

$$
\Phi(Z)=\sum_{j=0}^{\sqrt{R}-1} \tilde{c}_{j, \Phi}\left(Z_{2}-Z\right)^{j}+O\left(\left(Z_{2}-Z\right)^{\sqrt{R}}\right) .
$$

The asymptotic expansion is uniquely determined from the Eq. (3.56) and the first coefficient $\tilde{c}_{0, \Phi}$. We now recall that the function $\Phi_{1}$ belongs to $e^{\sqrt{R}}\left[0, Z_{a}\right]$ and $\Phi_{1}\left(Z_{2}\right)=1$. By adding $\Phi_{1}$ to the above expansion, we obtain another solution in which we can force the condition

$$
\tilde{c}_{0, \Phi}=c_{0, \Phi}
$$

with $c_{0, \Phi}$ appearing in (3.65). As a result, the asymptotic expansions of the inner and outer solutions are matched to order $\sqrt{R}$, so that the constructed solution is $C^{\sqrt{R}}$ at $Z_{2}$. Finally, we have shown that given any smooth function $H$ on $\left[0, Z_{a}\right]$, there exists a unique solution $\Phi$ to (3.56) on $\left[0, Z_{a}\right]$ which is $o\left(\left(Z_{2}-Z\right)^{-\frac{1}{c_{R}}}\right)$ at $Z=Z_{2}$. Furthermore, this solution is smooth for $Z \neq Z_{2}$ and $C^{\sqrt{R}}$ at $Z=Z_{2}$. In particular, with $T$ recovered by (3.54) and smooth for $Z \neq Z_{2}$ and $C^{\sqrt{R}-1}$ at $Z=Z_{2}$, we have that $(\Phi, T) \in \mathbb{H}_{2 k}$ for $R>R(k)$ large enough. Also, since $(\Phi, T)$ with $\Phi \sim\left(Z_{2}-Z\right)^{-\frac{1}{c_{R}}}$ near $Z=Z_{2}$ does not belong to $\mathbb{H}_{2 k}$, ${ }^{11}$ we have now proved that, in fact, there exists a unique solution $X=(\Phi, T)$ to $(-m+R) X=F$ on $\left[0, Z_{a}\right]$ in $\mathbb{H}_{2 k}$, which concludes the proof of (3.53).

Step 6 Density of $\mathscr{D}_{R}$. We now prove that $\mathscr{D}_{R}$ given by (3.48) is dense in $D(m)$. Indeed, if $X \in D(m)$, then $X \in \mathbb{H}_{2 k}$ and $m X \in \mathbb{H}_{2 k}$ so that there exists a sequence $\left(Y_{n}\right)_{n \in \mathbb{N}} \in C^{\infty}\left(\left[0, Z_{a}\right], \mathbb{C}^{2}\right)$ with

$$
\lim _{n \rightarrow+\infty} Y_{n} \rightarrow(-m+R) X \text { in } \mathbb{H}_{2 k}
$$

From step 5, for each integer $n$, there exist a unique $Z_{n} \in \mathscr{D}_{R}$ solution to

$$
(-m+R) Z_{n}=Y_{n}, \quad Z_{n} \in \mathbb{H}_{2 k},
$$

and hence

$$
(-m+R) Z_{n} \rightarrow(-m+R) X \text { in } \mathbb{H}_{2 k} .
$$

${ }^{11}$ Recall that $\left(c_{R}\right)^{-1} \gtrsim R \gg 1$. 
Thus, to conclude, it remains to check that $Z_{n}$ converges to $X$ in $\mathbb{H}_{2 k}$. To this end, since $Z_{n} \in \mathscr{D}_{R}$, (3.46) holds for $Z_{n}-Z_{q}$ and thus:

$$
\begin{aligned}
& \Re\left\langle Y_{n}-Y_{q}, Z_{n}-Z_{q}\right\rangle=\Re\left\langle(-m+R)\left(Z_{n}-Z_{q}\right), Z_{n}-Z_{q}\right\rangle \\
& \quad=\Re\left\langle(-m+\mathcal{A})\left(Z_{n}-Z_{q}\right), Z_{n}-Z_{q}\right\rangle-\Re\left\langle\mathcal{A}\left(Z_{n}-Z_{q}\right), Z_{n}-Z_{q}\right\rangle \\
& \quad+R\left\|Z_{n}-Z_{q}\right\|_{\mathbb{H}_{2 k}}^{2} \\
& \quad \geq R\left\|Z_{n}-Z_{q}\right\|_{\mathbb{H}_{2 k}}^{2}-\Re\left\langle\mathcal{A}\left(Z_{n}-Z_{q}\right), Z_{n}-Z_{q}\right\rangle
\end{aligned}
$$

so that, since $\mathcal{A}$ is a bounded operator, we infer for $R$ sufficiently large

$$
\frac{R}{2}\left\|Z_{n}-Z_{q}\right\|_{\mathbb{H}_{2 k}} \leq\left\|Y_{n}-Y_{q}\right\|_{\mathbb{H}_{2 k}} .
$$

In view of the convergence of $\left(Y_{n}\right)$ in $\mathbb{H}_{2 k}$, we deduce that $Z_{n}$ is a Cauchy sequence in $\mathbb{H}_{2 k}$ and hence converges, i.e.

$$
\lim _{n \rightarrow+\infty} Z_{n} \rightarrow Z \text { in } \mathbb{H}_{2 k}, \quad Z \in \mathbb{H}_{2 k} .
$$

Since $(-m+R) Z_{n}$ converges to $(-m+R) X$ in $\mathbb{H}_{2 k}$, we infer

$$
(-m+R)(Z-X)=0 \text { in } \mathscr{D}^{\prime}\left(0, Z_{a}\right), \quad Z-X \in \mathbb{H}_{2 k} .
$$

The uniqueness statement in (3.53) applied for $F=0$ yields $Z=X$. Thus $Z_{n} \rightarrow X$ and $(-m+R) Z_{n} \rightarrow(-m+R) X$ in $\mathbb{H}_{2 k}$. Finally, we have obtained a sequence $Z_{n} \in \mathscr{D}_{R}$ such that $Z_{n} \rightarrow X$ in $D(M)$, and hence $\mathscr{D}_{R}$ is dense in $D(m)$ as claimed.

Step 7 Maximal accretivity. We have proved in steps 1 to 3 that (3.46) holds for $X \in \mathscr{D}_{R}$, i.e.

$$
\forall X \in \mathscr{D}_{R}, \quad \Re\langle(-m+\mathcal{A}) X, X\rangle \geq c^{*} a k\langle X, X\rangle .
$$

Since $\mathscr{D}_{R}$ is dense in $D(m)$, in view of step 6, we have

$$
\forall X \in \mathscr{D}(m), \quad \Re\langle(-m+\mathcal{A}) X, X\rangle \geq c^{*} a k\langle X, X\rangle,
$$

which concludes the proof of the accretivity property (3.46).

We now claim:

$$
\forall F \in \mathbb{H}_{2 k}, \quad \exists X \in D(m) \text { such that }(-m+R) X=F .
$$

Indeed, since $F \in \mathbb{H}_{2 k}$, by density, there exists

$$
\lim _{n \rightarrow+\infty} F_{n} \rightarrow F \text { in } \mathbb{H}_{2 k}, \quad F_{n} \in C^{\infty}\left(\left[0, Z_{a}\right]\right) .
$$


Since $F_{n} \in C^{\infty}\left(\left[0, Z_{a}\right]\right)$, by (3.53), there exists $X_{n} \in \mathbb{H}_{2 k}$-solution to

$$
(-m+R) X_{n}=F_{n}
$$

Using (3.46) and arguing as in step 6, we have for $R$ sufficiently large

$$
\frac{R}{2}\left\|X_{n}-X_{q}\right\|_{\mathbb{H}_{2 k}} \leq\left\|F_{n}-F_{q}\right\|_{\mathbb{H}_{2 k}} .
$$

In view of the convergence of $\left(F_{n}\right)$ in $\mathbb{H}_{2 k}$, we deduce that $X_{n}$ is a Cauchy sequence in $\mathbb{H}_{2 k}$ and hence converges, i.e.

$$
\lim _{n \rightarrow+\infty} X_{n} \rightarrow X \text { in } \mathbb{H}_{2 k}, \quad X \in \mathbb{H}_{2 k}
$$

On the other hand, since $(-m+R) X_{n}=F_{n}$ converges to $F$ in $\mathbb{H}_{2 k}$, we infer

$$
(-m+R) X=F, \quad X \in D(m)
$$

which concludes the proof of (3.66).

Finally, (3.46) and a classical and elementary argument ${ }^{12}$ ensures that the maximality property (3.47) is implied by

$$
\exists R>0, \quad \forall F \in \mathbb{H}_{2 k}, \quad \exists X \in \mathscr{D}(m) \text { such that }(-\tilde{m}+R) X=F .
$$

Indeed, let $R>0$ large enough and $F \in \mathbb{H}_{2 k}$. Since $\mathcal{A}$ is a bounded operator, for $R$ large enough, from (3.66) and (3.46),

$$
\Re\langle F, X\rangle=\Re\langle(-m+R) X, X\rangle=\Re\langle(-\tilde{m}-\mathcal{A}+R) X, X\rangle \geq \frac{R}{2}\|X\|_{\mathbb{H}_{2 k}}^{2} .
$$

Therefore, for any $F \in \mathbb{H}_{2 k}$, solution $X$ to (3.66) is unique. Therefore, $(-m+R)^{-1}$ is well defined on $\mathbb{H}_{2 k}$ with the bound

$$
\left\|(-m+R)^{-1}\right\|_{\mathcal{L}\left(\mathbb{H}_{2 k}, \mathbb{H}_{2 k}\right)} \lesssim \frac{1}{R}
$$

Hence

$$
-\tilde{m}+R=-m+\mathcal{A}+R=(-m+R)\left[\operatorname{Id}+(-m+R)^{-1} \mathcal{A}\right]
$$

is invertible on $\mathbb{H}_{2 k}$ for $R$ large enough, which yields (3.47). This concludes the proof of Proposition 3.10.

\footnotetext{
12 More precisely, one can easily prove that the set of $R$ in $(0,+\infty)$ such that a solution $X$ exists is both closed and open. Hence, it suffices to prove that it is non empty.
} 


\section{Set up and the bootstrap}

In this section we describe a set of smooth well localized initial data which lead to the conclusions of Theorem 1.1. The heart of the proof is a bootstrap argument coupled to the classical Brouwer topological argument of Lemma 3.5 to avoid finitely many unstable directions of the corresponding linear flow. Since our analysis relies essentially on the phase-modulus decomposition of solutions of the Schrödinger equation, our chosen data needs to give rise to nowhere vanishing solutions to (1.1) (at least for a sufficiently small time as in Proposition 4.1 of [9]).

\subsection{Renormalized variables}

Let $u(t, x) \in \mathcal{C}\left(\left[0, T_{*}\right), \cap_{k \geq 0} H^{k}\right)$ be a solution to (1.1) such that $u(t, x)$ does not vanish at any $(t, x) \in\left[0, T_{*}\right) \times \mathbb{R}^{d}$. This will be a consequence of our choice of initial data and suitable bootstrap assumptions. We introduce for such a solution the decomposition of Lemma 2.1

$$
u(t, x)=\frac{1}{(\lambda \sqrt{b})^{\frac{2}{p-1}}} w(\tau, y) e^{i \gamma}, \quad w(\tau, y)=\rho_{\mathrm{Tot}}(\tau, Z) e^{i \frac{\Psi_{\mathrm{Tot}}}{b}}
$$

with the renormalized space and times

$$
\mid \begin{aligned}
& Z=y \sqrt{b}=Z^{*} x, \quad Z^{*}=e^{\mu \tau}, \\
& \lambda(\tau)=e^{-\frac{\tau}{2}}, \quad b(\tau)=e^{-e \tau}, \quad \gamma_{\tau}=-\frac{1}{b}=-e^{e \tau}, \\
& \tau=-\log \left(T_{*}-t\right), \quad \tau_{0}=-\log \left(T_{*}\right) .
\end{aligned}
$$

Here, $0<e<1$ is the fixed front speed such that

$$
r=\frac{2}{1-e}>2 .
$$

Up to a constant the phase can more explicitly be written in the form

$$
\gamma(\tau)=-\frac{1}{e b}
$$

Our claim is that given

$$
\tau_{0}=-\log \left(T_{*}\right)
$$

large enough, we can construct a finite co-dimensional manifold of smooth well localized initial data $u_{0}$ such that the corresponding solution to the renormalized flow (2.23) is global in renormalized time $\tau \in\left[\tau_{0},+\infty\right)$, bounded in a 
suitable topology and nowhere vanishing. Upon unfolding (4.1), this produces a solution to (1.1) blowing up at $T_{*}$ in the regime described by Theorem 1.1.

\subsection{Stabilization and regularization of the profile outside the singularity}

The spherically symmetric profile solution $\left(\rho_{P}, \Psi_{P}\right)$ has an intrinsic slow decay as $Z \rightarrow+\infty$

$$
\rho_{P}(Z)=\frac{c_{P}}{Z^{\frac{2(r-1)}{p-1}}}\left(1+O\left(\frac{1}{Z^{r}}\right)\right)
$$

which needs to be regularized in order to produce finite energy non vanishing initial data.

1. Stabilization of the profile. Recall the asymptotics (2.20) and the choice of parameters (4.3), (4.2) which yield

$$
\lambda^{2(r-2)}=b^{r}, \quad r=\frac{2}{1-e}, \quad \mu=\frac{1-e}{2} .
$$

For $Z=\frac{\sqrt{b}}{\lambda} x \gg 1$, i.e., outside the singularity:

$$
\begin{aligned}
u_{P}(t, x) & =\frac{e^{i \gamma(\tau)}}{(\lambda \sqrt{b})^{\frac{2}{p-1}}} \rho_{P}(Z) e^{i \frac{\Psi_{P}}{b}} \\
& =\frac{c_{P} e^{-\frac{i}{e b}}}{(\lambda \sqrt{b})^{\frac{2}{p-1}}\left(\frac{\sqrt{b}}{\lambda} x\right)^{\frac{2(r-1)}{p-1}} e^{i\left[\frac{1}{e b}+\frac{c_{\Psi}}{b\left(\frac{\sqrt{b}}{\lambda}\right)^{r-2}}\right]}\left(1+O\left(\frac{1}{\langle Z\rangle^{r}}\right)\right)} \\
& =\frac{c_{P}}{x^{\frac{2(r-1)}{p-1}}} e^{i \frac{c_{\Psi}}{x^{r-2}}\left[1+O\left(\frac{1}{Z^{r}}\right)\right]}\left[1+O\left(\frac{1}{Z^{r}}\right)\right]
\end{aligned}
$$

We see that far away from the singularity the profile $u_{P}$ is stationary. It is precisely this property that will allow us to dampen the tail of the profile below and construct solutions arising from rapidly decaying (in particular, finite energy) initial data.

2. Dampening of the tail. We dampen the tail outside the singularity $x \geq 1$, i.e., $Z \geq Z^{*}$ as follows. Let

$$
R_{P}(t, x)=\frac{1}{(\lambda \sqrt{b})^{\frac{2}{p-1}}} \rho_{P}(Z), \quad x=Z e^{-\mu \tau},
$$


then the asympotics (4.4) imply the existence of a limiting profile for $x \geq 1$ :

$$
R_{P}(t, x)=\frac{c_{P}}{x^{\frac{2(r-1)}{p-1}}}\left(1+O\left(e^{-\mu r \tau}\right)\right)
$$

We then pick once and for all a large integer $n_{P} \gg 1$ and define a smooth non decreasing connection $\mathscr{K}(x)$

$$
\mathscr{K}(x)=\mid \begin{aligned}
& 0 \text { for }|x| \leq 5, \\
& n_{P}-\frac{2(r-1)}{p-1} \text { for }|x| \geq 10
\end{aligned}
$$

for some large enough universal constant

$$
n_{P}=n_{P}(d) \gg 1 \text {. }
$$

We then define the dampened tail profile in original variables

$$
\begin{aligned}
R_{D}(t, x) & =R_{P}(t, x) e^{-\int_{0}^{x} \frac{\mathscr{K}\left(x^{\prime}\right)}{x^{\prime}} d x^{\prime}} \\
& =\mid \begin{array}{l}
R_{P}(t, x) \text { for }|x| \leq 5, \\
\left.\frac{c_{P}}{x^{n} P}\left[1+O\left(e^{-\mu r \tau}\right)\right)\right] \text { for }|x| \geq 10
\end{array},
\end{aligned}
$$

and hence in renormalized variables:

$$
\mid \begin{aligned}
& \rho_{D}(\tau, Z)=(\lambda \sqrt{b})^{\frac{2}{p-1}} R_{D}(t, x) \\
& x=\frac{Z}{Z^{*}}, Z^{*}=e^{\mu \tau}
\end{aligned}
$$

Let

$$
\zeta(x)=e^{-\int_{0}^{x} \frac{\mathscr{K}\left(x^{\prime}\right)}{x^{\prime}} d x^{\prime}},
$$

we have the equivalent representation:

$$
\begin{aligned}
\rho_{D}(Z) & =(\lambda \sqrt{b})^{\frac{2}{p-1}} R_{D}(\tau, x)=(\lambda \sqrt{b})^{\frac{2}{p-1}} R_{P}(t, x) \zeta(x) \\
& =\zeta\left(\frac{Z}{Z^{*}}\right) \rho_{P}(Z)
\end{aligned}
$$

Note that by construction for $j \in \mathbb{N}^{*}$ :

$$
-\frac{Z^{j} \partial_{Z}^{j} \rho_{D}}{\rho_{D}}=\mid \begin{aligned}
& (-1)^{j-1}\left(\frac{2(r-1)}{p-1}\right)^{j}+O\left(\frac{1}{\langle Z\rangle^{r}}\right) \text { for } Z \leq 5 Z^{*} \\
& (-1)^{j-1} n_{P}^{j}+O\left(\frac{1}{\langle Z\rangle^{r}}\right) \text { for } Z \geq 10 Z^{*}
\end{aligned}
$$


and

$$
\left|\frac{Z^{j} \partial_{j} \rho_{D}}{\rho_{D}}\right|_{L^{\infty}} \lesssim 1
$$

The obtained dampened profile for $Z \geq Z^{*}$ will be denoted

$$
\left(\rho_{D}, \Psi_{P}\right), Q_{D}=\rho_{D}^{p-1} .
$$

\subsection{Initial data}

We now describe explicitly an open set of initial data which will be considered as perturbations of the profile $\left(\rho_{D}, \Psi_{P}\right)$ in a suitable topology. The conclusions of Theorem 1.1 will hold for a finite co-dimension set of such data.

We pick universal constants $0<a \ll 1, Z_{0} \gg 1$ which will be adjusted along the proof and depend only on $(d, \ell)$. We define two levels of regularity

$$
\frac{d}{2} \ll k_{0} \ll k_{m},
$$

where $k_{m}$ denotes the maximum level of regularity required for the solution and $k_{0}$ is the level of regularity required for the linear spectral theory on the compact set $\left[0, Z_{a}\right]$.

0 . Variables and notations for derivatives. We define the variables

$$
\mid \begin{aligned}
& \rho_{\mathrm{Tot}}=\rho_{P}+\rho=\rho_{D}+\tilde{\rho}, \\
& \Psi_{\mathrm{Tot}}=\Psi_{P}+\Psi \\
& \Phi=\rho_{P} \Psi
\end{aligned}
$$

and specify the data in the $(\tilde{\rho}, \Psi)$ variables. We will use the following notations for derivatives. Given $k \in \mathbb{N}$, we note

$$
\partial^{k}=\left(\partial_{1}^{k}, \ldots, \partial_{d}^{k}\right), \quad f^{(k)}:=\partial^{k} f
$$

the vector of $k$-th derivatives in each direction. The notation $\partial_{Z}^{k} f$ is the $k$-th radial derivative. We let

$$
\tilde{\rho}_{k}=\Delta^{k} \tilde{\rho}, \quad \Psi_{k}=\Delta^{k} \Psi
$$

Given a multiindex $\alpha=\left(\alpha_{1}, \ldots, \alpha_{d}\right) \in \mathbb{N}^{d}$, we note

$$
\partial^{\alpha}=\partial_{1}^{\alpha_{1}} \cdots \partial_{d}^{\alpha_{d}}, \quad|\alpha|=\alpha_{1}+\cdots+\alpha_{d} .
$$


1. Initializing the Brouwer argument. We define the variables adapted to the spectral analysis according to (3.8), (3.12):

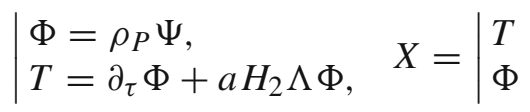

and recall the scalar product (3.44). For $0<c_{g}, a \ll 1$ small enough, we choose $k_{0} \gg 1$ such that Proposition 3.10 applies in the Hilbert space $\mathbb{H}_{2 k_{0}}$ with the spectral gap

$$
\forall X \in \mathscr{D}(m), \quad \Re\langle(-m+\mathcal{A}) X, X\rangle \geq c_{g}\langle X, X\rangle .
$$

Hence

$$
m=\left(m-\mathcal{A}+c_{g}\right)-c_{g}+\mathcal{A}
$$

and we may apply Lemma 3.4:

$$
\begin{aligned}
\Lambda_{0} & =\{\lambda \in \mathbb{C}, \quad \Re(\lambda) \geq 0\} \cap\{\lambda \text { is an eigenvalue of } m\} \\
& =\left(\lambda_{i}\right)_{1 \leq i \leq N}
\end{aligned}
$$

is a finite set corresponding to unstable eigenvalues, $V$ is an associated (unstable) finite dimensional invariant set, $U$ is the complementary (stable) invariant set

$$
\mathbb{H}_{2 k_{0}}=U \bigoplus V
$$

and $\mathbb{P}$ is the associated projection on $V$. We denote by $n$ the nilpotent part of the matrix, which consists of a finite collection of Jordan blocks, representing $m$ on $\mathrm{V}$ :

$$
\left.m\right|_{V}=n+\text { diag. }
$$

Note that $n$ commutes with $\left.m\right|_{V}$. Then there exist $C, \delta_{g}>0$ such that (3.5) holds:

$$
\forall X \in U,\left\|e^{\tau m} X\right\|_{\mathbb{H}_{2 k_{0}}} \leq C e^{-\frac{\delta_{g}}{2} \tau}\|X\|_{\mathbb{H}_{2 k_{0}}}, \quad \forall \tau \geq \tau_{0} .
$$

We now choose the data at $\tau_{0}$ such that (its restriction to $\left[0, Z_{a}\right]$, where the projection $\mathbb{P}$ and the space $\mathbb{H}_{2 k_{0}}$ are defined, satisfies)

$$
\left\|(I-\mathbb{P}) X\left(\tau_{0}\right)\right\|_{\mathbb{H}_{2 k_{0}}} \leq e^{-\frac{\delta_{g}}{2} \tau_{0}}, \quad\left\|\mathbb{P} X\left(\tau_{0}\right)\right\|_{\mathbb{H}_{2 k_{0}}} \leq e^{-\frac{3 \delta_{g}}{5} \tau_{0}}
$$


2. Bounds on local low Sobolev norms. Let $0 \leq m \leq 2 k_{0}$ and

$$
v_{0}=-\frac{2(r-1)}{p-1}+\frac{\delta_{g}}{2 \mu}
$$

let the weight function

$$
\chi_{v_{0}, m}=\frac{1}{\langle Z\rangle^{d-2(r-1)+2\left(\nu_{0}-m\right)}} \zeta\left(\frac{Z}{Z^{*}}\right), \quad \zeta(Z)=\mid \begin{aligned}
& 1 \text { for } Z \leq 2 \\
& 0 \text { for } Z \geq 3 .
\end{aligned}
$$

Then

$$
\sum_{m=0}^{2 k_{0}} \int(p-1) Q\left(\partial^{m} \rho\left(\tau_{0}\right)\right)^{2} \chi_{\nu_{0}, m}+\left|\nabla \partial^{m} \Phi\left(\tau_{0}\right)\right|^{2} \chi_{\nu_{0}, m} \leq e^{-\delta_{g} \tau_{0}}
$$

4. Pointwise assumptions. We assume the following interior pointwise bounds

$$
\begin{aligned}
\forall 0 & \leq k \leq k_{m}+1 \\
& \left\|\frac{\langle Z\rangle^{k} \partial_{Z}^{k} \tilde{\rho}\left(\tau_{0}\right)}{\rho_{D}}\right\|_{L^{\infty}\left(Z \leq Z^{*}\right)}+\left\|\langle Z\rangle^{r-2}\langle Z\rangle^{k} \partial_{Z}^{k} \Psi\left(\tau_{0}\right)\right\|_{L^{\infty}\left(Z \leq Z^{*}\right)} \\
& \leq b_{0}^{c_{0}}
\end{aligned}
$$

for some small enough universal constant $c_{0}$, and the exterior bounds:

$$
\begin{aligned}
\forall 0 & \leq k \leq k_{m}+1, \\
& \left\|\frac{Z^{k+1} \partial_{Z}^{k} \tilde{\rho}\left(\tau_{0}\right)}{\rho_{D}}\right\|_{L^{\infty}\left(Z \geq Z^{*}\right)}+\frac{\left\|Z^{k+1} \partial_{Z}^{k} \Psi\left(\tau_{0}\right)\right\|_{L^{\infty}\left(Z \geq Z^{*}\right)}}{b_{0}} \\
& \leq b_{0}^{C_{0}}
\end{aligned}
$$

for some large enough universal $C_{0}(d, r, p)$. Note in particular that (4.20), (4.21) ensure for $0<b_{0}<b_{0}^{*} \ll 1$ small enough:

$$
\left\|\frac{\tilde{\rho}\left(\tau_{0}\right)}{\rho_{D}}\right\|_{L^{\infty}} \leq \delta_{0} \ll 1
$$

and hence the data does not vanish.

5. Global rough bound for large Sobolev norms. We consider the global Sobolev norm 


$$
\begin{aligned}
& \|\tilde{\rho}, \Psi\|_{k_{m}}^{2} \\
& \quad:=\sum_{j=0}^{k_{m}} \sum_{|\alpha|=j} \int \frac{b^{2}\left|\nabla \partial^{\alpha} \tilde{\rho}\right|^{2}+(p-1) \rho_{D}^{p-2} \rho_{\mathrm{Tot}}\left(\partial^{\alpha} \tilde{\rho}\right)^{2}+\rho_{\mathrm{Tot}}^{2}\left|\nabla \partial^{\alpha} \Psi\right|^{2}}{\langle Z\rangle^{2\left(k_{m}-j\right)}},
\end{aligned}
$$

then we require:

$$
\left\|\tilde{\rho}\left(\tau_{0}\right), \Psi\left(\tau_{0}\right)\right\|_{k_{m}} \leq \frac{1}{2} .
$$

The bound above is actually implied by the pointwise assumptions.

Remark 4.1 Note that we may without loss of generality assume $u_{0} \in$ $\cap_{k \geq 0} H^{k}$.

\subsection{Bootstrap bounds}

We make the following bootstrap assumptions on the maximal interval $\left[\tau_{0}, \tau^{*}\right)$. 0 . Non vanishing and hydrodynamical variables. From standard Cauchy theory and the smoothness of the nonlinearity since $p \in 2 \mathbb{N}^{*}+1$, the smooth data $u_{0} \in \cap_{k \geq 0} H^{k}$ generates a unique local solution $u \in C\left(\left[0, T_{*}\right), \cap_{k \geq 0} H^{k}\right)$ with the blow up criterion

$$
T_{*}<+\infty \Rightarrow \lim _{t \rightarrow T_{*}}\|u(t, \cdot)\|_{H^{k_{c}}}=+\infty
$$

for some large enough $k_{c}(d, p)$. To ensure non vanishing, we first note that since $\inf _{|x| \leq 10}\left|u_{0}(x)\right|>0$, the continuity of $u$ in time ensures $\inf _{|x| \leq 10}|u(t, x)|>0$ for $t \in\left[0, T_{*}\right], T_{*}>0$ small enough. For $|x| \geq 10$, we estimate from the flow

$$
\left|r^{n_{P}}\right| u(t, x)\left|-r^{n_{P}}\right| u_{0}|| \leq\left.\int_{0}^{t} r^{n_{P}}|\Delta u-u| u\right|^{p-1} \mid d t
$$

and hence from our choice of initial data, the non vanishing of $u(t, x)$ follows on a time interval where

$$
T_{*}\left\|r^{n_{P}}\left(|\Delta u|+|u|^{p}\right)\right\|_{L^{\infty}\left(\left[0, T_{*}\right),|x| \geq 10\right)} \leq \delta
$$

for some sufficiently small universal constant $0<\delta \ll 1$. Using spherical symmetry we can replace the above by 


$$
\begin{aligned}
& T_{*}\left(\left\|\langle x\rangle^{n_{P}+1-\frac{d}{2}+\epsilon} \Delta u\right\|_{L^{\infty}\left(\left[0, T_{*}\right) ; H^{1}\right)}\right. \\
& \left.\quad+\left\|r^{2 \epsilon} u\right\|_{L^{\infty}\left(\left[0, T_{*}\right),|x| \geq 10\right)}^{p-1}\left\|\langle x\rangle^{n_{P}+1-\frac{d}{2}-\epsilon} u\right\|_{L^{\infty}\left(\left[0, T_{*}\right) ; H^{1}\right)}\right) \leq \delta
\end{aligned}
$$

for an arbitrarily small $\epsilon>0$. Our initial data $u_{0}$ belongs to the space

$$
\cap_{k \geq 0} H^{k} \cap\left\|\langle x\rangle^{n_{P}+1-\frac{d}{2}-\epsilon} u\right\|_{L^{2}} \cap\left\|\langle x\rangle^{n_{P}+3-\frac{d}{2}-\epsilon} \Delta u\right\|_{L^{2}} .
$$

Existence of the desired time interval $\left[0, T_{*}\right.$ ) now follows from a local wellposedness for NLS in weighted Sobolev spaces which is (essentially) in [27].

We may therefore introduce the hydrodynamical variables (4.1) on such a small enough time interval and will bootstrap the smallness bound which ensures non vanishing:

$$
\left\|\frac{\tilde{\rho}}{\rho_{\mathrm{Tot}}}\right\|_{L^{\infty}} \leq \delta
$$

for some sufficiently small $0<\delta=\delta\left(k_{m}\right) \ll 1$.

1. Global weighted Sobolev norms. Pick a small enough universal constant $0<\tilde{v}<\tilde{v}^{*}\left(k_{m}\right) \ll 1$, we define

$$
\mid \begin{aligned}
& v=\tilde{v}-\frac{2(r-1)}{p-1} \\
& \sigma_{v}=v+\frac{d}{2}-(r-1) \\
& m_{0}=\frac{4 k_{m}}{9}+1
\end{aligned}
$$

and let the continuous function:

$$
\sigma(m)=\mid \begin{aligned}
& \sigma_{v}-m \text { for } 0 \leq m \leq m_{0}, \\
& -\alpha\left(k_{m}-m\right) \text { for } m_{0} \leq m \leq k_{m}
\end{aligned}
$$

with the continuity requirement at $m_{0}$ :

$$
\alpha\left(k_{m}-m_{0}\right)=m_{0}-\sigma_{v}, \quad \alpha=\frac{m_{0}-\sigma_{v}}{k_{m}-m_{0}}=\frac{4}{5}+O\left(\frac{1}{k_{m}}\right) .
$$

In particular, $\alpha<1$. We note that for all $1 \leq m \leq k_{m}$

$$
\sigma(m-1) \geq \sigma(m)-\alpha .
$$

We also define the function

$$
\tilde{\sigma}(k)=\mid \begin{aligned}
& n_{P}-\frac{2(r-1)}{p-1}-(r-2)+2 \tilde{v} \text { for } 0 \leq k \leq \frac{2 k_{m}}{3}+1, \\
& \beta\left(k_{m}-k\right) \text { for } \frac{2 k_{m}}{3}+1 \leq k \leq k_{m},
\end{aligned}
$$




$$
\leq n_{P}-\frac{2(r-1)}{p-1}-(r-2)+2 \tilde{v},
$$

where $\beta$ is computed through the continuity requirement at $\frac{2 k_{m}}{3}$ :

$$
\frac{k_{m}}{3} \beta=n_{P}-\frac{2(r-1)}{p-1}-(r-2)+2 \tilde{v} \Leftrightarrow \beta=3 \frac{n_{P}-\frac{2(r-1)}{p-1}-(r-2)+2 \tilde{v}}{k_{m}} .
$$

We will choose $n_{P} \ll k_{m}$, e.g. $n_{P}=\frac{k_{m}}{30}$, so that in particular,

$$
\beta<\frac{1}{10}, \quad \alpha+\beta \leq 1
$$

We also note that

$$
\tilde{\sigma}(m-1) \leq \tilde{\sigma}(m)+\beta .
$$

We then define the weighted Sobolev norm:

$$
\mid \begin{aligned}
& \|\tilde{\rho}, \Psi\|_{m, \sigma(m)}^{2}=\sum_{k=0}^{m} \int \chi_{m, k, \sigma(m)} \\
& {\left[b^{2}\left|\nabla \tilde{\rho}^{(k)}\right|^{2}+(p-1) \rho_{D}^{p-2} \rho_{\mathrm{Tot}}\left(\tilde{\rho}^{(k)}\right)^{2}+\rho_{\mathrm{Tot}}^{2}\left|\nabla \Psi^{(k)}\right|^{2}\right],} \\
& \chi_{m, k, \sigma(m)}(Z)=\frac{1}{\langle Z\rangle^{2(m-k+\sigma(m))}} \xi_{m}\left(\frac{Z}{Z^{*}}\right),
\end{aligned}
$$

where the function

$$
\xi_{m}(x)=\mid \begin{array}{ll}
1 & \text { for } x \leq 1 \\
x^{2 \tilde{\sigma}(m)} & \text { for } x>1
\end{array}
$$

We assume the bootstrap bound:

$$
\|\tilde{\rho}, \Psi\|_{m, \sigma(m)}^{2} \leq 1, \quad 0 \leq m \leq k_{m}-1 .
$$

Remark 4.2 (Equivalence of norms) It is easy to see that the norm (4.33) is equivalent

$$
\begin{aligned}
\|\tilde{\rho}, \Psi\|_{m, \sigma(m)}^{2} \approx & \sum_{k=0}^{m} \sum_{|\alpha|=k} \int \chi_{m, k, \sigma(m)} \\
& {\left[b^{2}\left|\nabla \nabla^{\alpha} \tilde{\rho}\right|^{2}+\rho_{D}^{p-1}\left|\nabla^{\alpha} \tilde{\rho}\right|^{2}+\rho_{D}^{2}\left|\nabla \nabla^{\alpha} \Psi\right|^{2}\right] }
\end{aligned}
$$


and for even $m$

$$
\begin{aligned}
\|\tilde{\rho}, \Psi\|_{m, \sigma(m)}^{2} \approx & \sum_{k=0}^{\frac{m}{2}} \int \chi_{m, 2 k, \sigma(m)} \\
& {\left[b^{2}\left|\nabla \tilde{\rho}_{k}\right|^{2}+\rho_{D}^{p-1}\left|\tilde{\rho}_{k}\right|^{2}+\rho_{D}^{2}\left|\nabla \Psi_{k}\right|^{2}\right] . }
\end{aligned}
$$

Let us briefly sketch the proof. First, we note that the weight function $\chi_{m, k, \sigma}$ can be replaced by a smooth function $\tilde{\chi}_{m, k, \sigma}$ with similar properties. In particular,

$$
\left|\nabla^{\alpha} \tilde{\chi}_{m, k, \sigma}\right| \leq C_{\alpha, m, k, \sigma} \frac{\tilde{\chi}_{m, k, \sigma}}{\langle Z\rangle^{\alpha}}
$$

The functions $\rho_{D}^{2} \tilde{\chi}_{m, k, \sigma}$ and $\rho_{D}^{p-1} \tilde{\chi}_{m, k, \sigma}$ also obey the property above. We now consider the case $m=2$, let $\hat{\chi}$ be a weight function obeying (4.37) and observe that

$$
\int \hat{\chi}\left|\partial_{1} \partial_{2} f\right|^{2}=\int \hat{\chi} \partial_{1}^{2} f \partial_{2}^{2} f-\int \partial_{1} \hat{\chi} \partial_{2} f \partial_{1} \partial_{2} f+\int \partial_{2} \hat{\chi} \partial_{2} f \partial_{1}^{2} f .
$$

Therefore,

$$
\int \hat{\chi}\left|\partial_{1} \partial_{2} f\right|^{2} \lesssim \int \hat{\chi}\left(\left|\partial_{1}^{2} f\right|^{2}+\left|\partial_{2}^{2} f\right|^{2}\right)+\int \frac{\hat{\chi}}{\langle Z\rangle^{2}}\left(\left|\partial_{1} f\right|^{2}+\left|\partial_{2} f\right|^{2}\right) .
$$

Using this for $f=\nabla \tilde{\rho}, \tilde{\rho}, \nabla \Psi$ and with any mixed derivative in place of $\partial_{1} \partial_{2}$ immediately confirms the equivalence of the norms (4.33) and (4.35) for $m=2$. The equivalence for higher derivatives can be proved by induction. The equivalence with (4.36) follows from a similar Bochner type identity

$$
\begin{aligned}
\sum_{i, j=1}^{d} \int \hat{\chi}\left|\partial_{i} \partial_{j} f\right|^{2}= & \int \hat{\chi}|\Delta f|^{2}-\sum_{i, j=1}^{d} \int \partial_{i} \hat{\chi} \partial_{j} f \partial_{i} \partial_{j} f \\
& +\sum_{i, j=1}^{d} \int \partial_{j} \hat{\chi} \partial_{j} f \partial_{i} \partial_{i} f
\end{aligned}
$$

implying

$$
\begin{aligned}
\sum_{i, j=1}^{d} \int \hat{\chi}\left|\partial_{i} \partial_{j} f\right|^{2} & \lesssim \int \hat{\chi}|\Delta f|^{2}+\int \frac{\hat{\chi}}{\langle Z\rangle^{2}}|\nabla f|^{2} \\
& \lesssim \int \hat{\chi}|\Delta f|^{2}+\int \frac{\hat{\chi}}{\langle Z\rangle^{4}}|f|^{2}
\end{aligned}
$$


This gives the equivalence of (4.33) and (4.36) for $m=2$. Once again, higher norms follow by induction.

Finally, note that the above norm equivalences are even independent of the assumption of spherical symmetry on $\tilde{\rho}, \Psi$.

2. Global control of the highest Sobolev norm:

$$
\|\tilde{\rho}, \Psi\|_{k_{m}}^{2}=\|\tilde{\rho}, \Psi\|_{k_{m}, \sigma(m)}^{2} \leq 1 .
$$

3. Local decay of low Sobolev norms: for any $0 \leq k \leq 2 k_{0}$, any $1 \leq \hat{Z} \leq Z^{*}$ and universal constant $C=C\left(k_{0}\right)$ :

$$
\|(\tilde{\rho}, \Psi)\|_{H^{k}(Z \leq \hat{Z})} \leq \hat{Z}^{C} e^{-\frac{3 \delta g}{8} \tau} .
$$

4. Pointwise bounds:

$$
\mid \begin{aligned}
& \forall 0 \leq k \leq \frac{2 k_{m}}{3}, \quad\left\|\frac{Z^{n(k)} \partial_{Z}^{k} \tilde{\rho}}{\rho_{D}}\right\|_{L^{\infty}} \leq 1, \\
& \forall 1 \leq k \leq \frac{2 k_{m}}{3}, \quad\left\|Z^{n(k)}\langle Z\rangle^{r-2} \partial_{Z}^{k} \Psi\right\|_{L^{\infty}\left(Z \leq Z^{*}\right)}+\frac{\left\|Z^{n(k)} \partial_{Z}^{k} \Psi\right\|_{L^{\infty}\left(Z \geq Z^{*}\right)}}{b} \leq 1
\end{aligned}
$$

with

$$
n(k)=\mid \begin{array}{ll}
k & \text { for } \quad k \leq \frac{4 k_{m}}{9} \\
\frac{k_{m}}{4} & \text { for } \quad \frac{4 k_{m}}{9}<k \leq \frac{2 k_{m}}{3} .
\end{array}
$$

Remark 4.3 Since $b=e^{-\mu(r-2) \tau},(4.20)$ and (4.21) imply that the initial data verify the bootstrap inequalities (4.34), (4.38), (4.40) with the bound $e^{-c \tau_{0}}$ for some small universal constant $c$.

The heart of the proof of Theorem 1.1 is the following:

Proposition 4.4 (Bootstrap) Let $\tau^{*}$ be the maximal time with property that (see (4.16) for the definition of $n$ )

$$
\| e^{-\tau n_{\mathbb{P}} X(\tau) \|_{\mathbb{H}_{2 k_{0}}}<e^{-\frac{19 \delta_{g}}{30} \tau}}
$$

for all $\tau \in\left[\tau_{0}, \tau^{*}\right)$ and that the bounds (4.26), (4.34), (4.38), (4.39), (4.40), (4.27) hold on $\left[\tau_{0}, \tau^{*}\right)$ with $\delta^{-1}, \tau_{0}$ large enough. Then the following holds: 1. Exit criterion. The bounds (4.26), (4.34), (4.38), (4.39), (4.40), (4.27) can be strictly improved ${ }^{13}$ on $\left[\tau_{0}, \tau^{*}\right)$. Consequently, either $\tau^{*}=+\infty$ or, if $\tau^{*}<$

\footnotetext{
13 More precisely, the same bounds hold with the corresponding constants on the right-hand side being replaced by $1 / 2$ of their values.
} 
$+\infty$, then

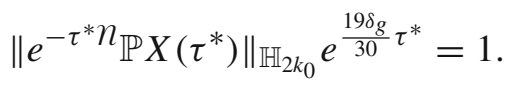

2. Linear evolution. The right hand side $G$ of the equation for $X(\tau)$

$$
\partial_{\tau} X=m X+G
$$

satisfies

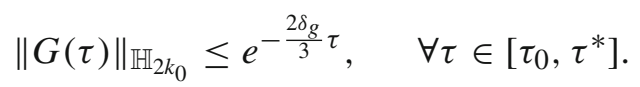

We will show in Sect. 8.3 that Proposition 4.4 immediately implies Theorem 1.1 .

Remark 4.5 We note that the assumption (4.42) implies that

$$
\|\mathbb{P} X(\tau)\|_{\mathbb{H}_{2 k_{0}}} \leq e^{-\frac{\delta g}{2} \tau}, \quad \forall \tau \in\left[\tau_{0}, \tau^{*}\right) .
$$

We will prove the bootstrap Proposition 4.4 under the weaker assumption (4.45). Specifically, we will define $\left[\tau_{0}, \tau^{*}\right)$ to be the maximal time interval on which (4.45) holds and will show that both the bounds (4.26), (4.34), (4.38), (4.39), (4.40), (4.27) can be improved and that $G$ satisfies (4.44).

We now focus on the proof of Proposition 4.4 and work on a time interval $\left[\tau_{0}, \tau^{*}\right), \tau_{0}<\tau^{*} \leq+\infty$ on which (4.26), (4.34), (4.38), (4.39), (4.40), (4.27) and (4.45) hold.

\section{Control of high Sobolev norms}

We first turn to the global in space control of high Sobolev norms. This is an essential step to control the $b$ dependence of the flow and the dissipative structure which can neither be treated by spectral analysis nor perturbatively. We claim an improvement of the bound (4.34), controlling all but the highest weighted Sobolev norm.

Proposition 5.1 There exists a universal constant $c_{k_{m}}^{*}>0$ such that for all $0 \leq m \leq k_{m}-1$

$$
\|\tilde{\rho}, \Psi\|_{m, \sigma(m)} \leq e^{-c_{k_{m}}^{*} \tau} .
$$

The rest of this section is devoted to the proof of Proposition 5.1. Let us outline the main steps: 
(1) First, we derive a general weighted energy identity, see (5.10), which will be used several times in the paper, and which respects the quasilinear structure of the problem. It is important that the $b^{2} \Delta$ term that was neglected in the Euler approximation of the flow produces a positive term in (5.10).

(2) Second, we show that thanks to our choice of weights, and knowing decay on the light cone, we can derive from (5.10) the differential inequality (5.13). The control of the corresponding nonlinear terms relies on classical interpolation estimates between weighted Sobolev norms.

\subsection{Algebraic energy identity}

We derive the energy identity for high Sobolev norms. Due to the use of the hydrodynamical variables, the identity exhibits a quasilinear structure.

Step 1 Equation for $\tilde{\rho}, \Psi$. Recall (2.23):

$$
\mid \begin{gathered}
\partial_{\tau} \rho_{\mathrm{Tot}}=-\rho_{\mathrm{Tot}} \Delta \Psi_{\mathrm{Tot}}-\frac{\mu \ell(r-1)}{2} \rho_{\mathrm{Tot}}-\left(2 \partial_{Z} \Psi_{\mathrm{Tot}}+\mu Z\right) \partial_{Z} \rho_{\mathrm{Tot}} \\
\rho_{\mathrm{Tot}} \partial_{\tau} \Psi_{\mathrm{Tot}}=b^{2} \Delta \rho_{\mathrm{Tot}}-\left[\left|\nabla \Psi_{\mathrm{Tot}}\right|^{2}+\mu(r-2) \Psi_{\mathrm{Tot}}-1\right. \\
\left.+\mu \Lambda \Psi_{\mathrm{Tot}}+\rho_{\mathrm{Tot}}^{p-1}\right] \rho_{\mathrm{Tot}} .
\end{gathered}
$$

By construction

$$
\mid \begin{aligned}
& \left|\nabla \Psi_{P}\right|^{2}+\rho_{D}^{p-1}+\mu(r-2) \Psi_{P}+\mu \Lambda \Psi_{P}-1=\tilde{\mathscr{E}}_{P, \Psi} \\
& \partial_{\tau} \rho_{D}+\rho_{D}\left[\Delta \Psi_{P}+\frac{\mu \ell(r-1)}{2}+\left(2 \partial_{Z} \Psi_{P}+\mu Z\right) \frac{\partial_{Z} \rho_{D}}{\rho_{D}}\right]=\tilde{\mathscr{E}}_{P, \rho}
\end{aligned}
$$

with $\tilde{\mathscr{E}}$ supported in $Z \geq 3 Z^{*}$. The linearized flow is given by

$$
\mid \begin{gathered}
\partial_{\tau} \tilde{\rho}=-\rho_{\mathrm{Tot}} \Delta \Psi-2 \nabla \rho_{\mathrm{Tot}} \cdot \nabla \Psi+H_{1} \tilde{\rho}-H_{2} \Lambda \tilde{\rho}-\tilde{\mathscr{E}}_{P, \rho} \\
\partial_{\tau} \Psi=b^{2} \frac{\Delta \rho_{\mathrm{Tot}}}{\rho_{\mathrm{Tot}}}-\left\{H_{2} \Lambda \Psi+\mu(r-2) \Psi+|\nabla \Psi|^{2}\right. \\
\left.\quad+(p-1) \rho_{D}^{p-2} \tilde{\rho}+\mathrm{NL}(\tilde{\rho})\right\}-\tilde{\mathscr{E}}_{P, \Psi}
\end{gathered}
$$

with the nonlinear term

$$
\mathrm{NL}(\tilde{\rho})=\left(\rho_{D}+\tilde{\rho}\right)^{p-1}-\rho_{D}^{p-1}-(p-1) \rho_{D}^{p-2} \tilde{\rho} .
$$

Note that the potentials

$$
H_{2}=\mu+2 \frac{\Psi_{P}^{\prime}}{Z}, \quad H_{1}=-\left(\Delta \Psi_{P}+\frac{\mu \ell(r-1)}{2}\right)
$$


remain the same in these equations: they are not affected by the profile localization introduced by passing from $\rho_{P}$ to $\rho_{D}$. We recall the Emden transform formulas (2.24):

$$
\mid \begin{aligned}
& H_{2}=\mu(1-w) \\
& H_{1}=\frac{\mu \ell}{2}(1-w)\left[1+\frac{\Lambda \sigma}{\sigma}\right]
\end{aligned}
$$

which, using (2.19), yield the bounds:

$$
\mid \begin{aligned}
& H_{2}=\mu+O\left(\frac{1}{\langle Z\rangle^{r}}\right), \quad H_{1}=-\frac{2 \mu(r-1)}{p-1}+O\left(\frac{1}{\langle Z\rangle^{r}}\right), \\
& \left|\langle Z\rangle^{j} \partial_{Z}^{j} H_{1}\right|+\left|\langle Z\rangle^{j} \partial_{Z}^{j} H_{2}\right| \lesssim \frac{1}{\langle Z\rangle^{r}}, \quad j \geq 1 .
\end{aligned}
$$

Our main task is now to produce an energy identity for (5.3) which respects the quasilinear nature of (5.3) and does not loose derivatives.

Step 2 Equation for derivatives. We recall the notation for the vector $\partial^{k}$ :

$$
\mid \begin{aligned}
& \partial^{k}:=\left(\partial_{1}^{k}, \ldots, \partial_{d}^{k}\right) \\
& \tilde{\rho}^{(k)}=\partial^{k} \tilde{\rho}, \quad \Psi^{(k)}=\partial^{k} \Psi
\end{aligned}
$$

Also, for convenience, we denote $\partial^{1}$ in various computations simply by $\partial$.

We use

$$
\left[\partial^{k}, \Lambda\right]=k \partial^{k}
$$

to compute from (5.3):

$$
\begin{aligned}
\partial_{\tau} \tilde{\rho}^{(k)}= & \left(H_{1}-k H_{2}\right) \tilde{\rho}^{(k)}-H_{2} \Lambda \tilde{\rho}^{(k)}-\left(\partial^{k} \rho_{\mathrm{Tot}}\right) \Delta \Psi-k \partial \rho_{\mathrm{Tot}} \partial^{k-1} \Delta \Psi \\
& -\rho_{\mathrm{Tot}} \Delta \Psi^{(k)}-2 \nabla\left(\partial^{k} \rho_{\mathrm{Tot}}\right) \cdot \nabla \Psi-2 \nabla \rho_{\mathrm{Tot}} \cdot \nabla \Psi^{(k)}+F_{1}
\end{aligned}
$$

with

$$
\begin{aligned}
& F_{1}=-\partial^{k} \tilde{\mathscr{E}}_{P, \rho}+\left[\partial^{k}, H_{1}\right] \tilde{\rho}-\left[\partial^{k}, H_{2}\right] \Lambda \tilde{\rho} \\
& -\quad \sum c_{j_{1}, j_{2}} \partial^{j_{1}} \rho_{\operatorname{Tot}} \partial^{j_{2}} \Delta \Psi \\
& \mid \begin{array}{l}
j_{1}+j_{2}=k \\
j_{1} \geq 2, j_{2} \geq 1
\end{array} \\
& -\sum_{\substack{j_{1}+j_{2}=k \\
j_{1}, j_{2} \geq 1}} c_{j_{1}, j_{2}} \partial^{j_{1}} \nabla \rho_{\mathrm{Tot}} \cdot \partial^{j_{2}} \nabla \Psi .
\end{aligned}
$$


For the second equation:

$$
\begin{aligned}
\partial_{\tau} \Psi^{(k)}= & b^{2}\left(\frac{\partial^{k} \Delta \rho_{\mathrm{Tot}}}{\rho_{\mathrm{Tot}}}-\frac{k \partial^{k-1} \Delta \rho_{\mathrm{Tot}} \partial \rho_{\mathrm{Tot}}}{\rho_{\mathrm{Tot}}^{2}}\right) \\
& -k H_{2} \Psi^{(k)}-H_{2} \Lambda \Psi^{(k)}-\mu(r-2) \Psi^{(k)}-2 \nabla \Psi \cdot \nabla \Psi^{(k)} \\
& -\left[(p-1) \rho_{D}^{p-2} \tilde{\rho}^{(k)}+k(p-1)(p-2) \rho_{D}^{p-3} \partial \rho_{D} \partial^{k-1} \tilde{\rho}\right]+F_{2}
\end{aligned}
$$

with

$$
\begin{aligned}
F_{2}= & -\partial^{k} \tilde{\mathscr{E}}_{P, \Psi}+b^{2}\left[\partial^{k}\left(\frac{\Delta \rho_{\mathrm{Tot}}}{\rho_{\mathrm{Tot}}}\right)-\frac{\partial^{k} \Delta \rho_{\mathrm{Tot}}}{\rho_{\mathrm{Tot}}}+\frac{k \partial^{k-1} \Delta \rho_{\mathrm{Tot}} \partial \rho_{\mathrm{Tot}}}{\rho_{\mathrm{Tot}}^{2}}\right] \\
& -\left[\partial^{k}, H_{2}\right] \Lambda \Psi-(p-1)\left(\left[\partial^{k}, \rho_{D}^{p-2}\right] \tilde{\rho}-k(p-2) \rho_{D}^{p-3} \partial \rho_{D} \partial^{k-1} \tilde{\rho}\right) \\
& -\sum_{j_{1}+j_{2}=k, j_{1}, j_{2} \geq 1} \partial^{j_{1}} \nabla \Psi \cdot \partial^{j_{2}} \nabla \Psi-\partial^{k} \operatorname{NL}(\tilde{\rho}) .
\end{aligned}
$$

Step 3 Algebraic energy identity. Let $\chi$ be a smooth function. We compute:

$$
\begin{aligned}
\frac{1}{2} \frac{d}{d \tau} & \left\{\int b^{2} \chi\left|\nabla \tilde{\rho}^{(k)}\right|^{2}+(p-1) \int \chi \rho_{D}^{p-2} \rho_{\mathrm{Tot}}\left(\tilde{\rho}^{(k)}\right)^{2}+\int \chi \rho_{\mathrm{Tot}}^{2}\left|\nabla \Psi^{(k)}\right|^{2}\right\} \\
= & \frac{1}{2} \int \partial_{\tau} \chi\left\{b^{2}\left|\nabla \tilde{\rho}^{(k)}\right|^{2}+(p-1) \rho_{D}^{p-2} \rho_{\mathrm{Tot}}\left(\tilde{\rho}^{(k)}\right)^{2}+\rho_{\mathrm{Tot}}^{2}\left|\nabla \Psi^{(k)}\right|^{2}\right\} \\
& -e b^{2} \int \chi\left|\nabla \tilde{\rho}^{(k)}\right|^{2}+\int \partial_{\tau} \tilde{\rho}^{(k)}\left[-b^{2} \chi \Delta \tilde{\rho}^{(k)}\right. \\
& \left.-b^{2} \nabla \chi \cdot \nabla \tilde{\rho}^{(k)}+(p-1) \chi \rho_{D}^{p-2} \rho_{\mathrm{Tot}} \tilde{\rho}^{(k)}\right] \\
& +\frac{p-1}{2} \int \chi(p-2) \partial_{\tau} \rho_{D} \rho_{D}^{p-3} \rho_{\mathrm{Tot}}\left(\tilde{\rho}^{(k)}\right)^{2} \\
& +\int \chi \partial_{\tau} \rho_{\mathrm{Tot}}\left[\frac{p-1}{2} \rho_{D}^{p-2}\left(\tilde{\rho}^{(k)}\right)^{2}+\rho_{\mathrm{Tot}}\left|\nabla \Psi^{(k)}\right|^{2}\right] \\
& -\int \partial_{\tau} \Psi^{(k)}\left[2 \chi \rho_{\mathrm{Tot}} \nabla \rho_{\mathrm{Tot}} \cdot \nabla \Psi^{(k)}\right. \\
& \left.+\chi \rho_{\mathrm{Tot}}^{2} \Delta \Psi^{(k)}+\rho_{\mathrm{Tot}}^{2} \nabla \chi \cdot \nabla \Psi^{(k)}\right] .
\end{aligned}
$$

We compute:

$$
\begin{aligned}
& \int \partial_{\tau} \tilde{\rho}^{(k)}\left[-b^{2} \chi \Delta \tilde{\rho}^{(k)}-b^{2} \nabla \chi \cdot \nabla \tilde{\rho}^{(k)}+(p-1) \chi \rho_{D}^{p-2} \rho_{\mathrm{Tot}} \tilde{\rho}^{(k)}\right] \\
& =\int F_{1}\left[-b^{2} \nabla \cdot\left(\chi \nabla \tilde{\rho}^{(k)}\right)+(p-1) \chi \rho_{D}^{p-2} \rho_{\mathrm{Tot}} \tilde{\rho}^{(k)}\right] \\
& \quad+\int\left[\left(H_{1}-k H_{2}\right) \tilde{\rho}^{(k)}-H_{2} \Lambda \tilde{\rho}^{(k)}-\left(\partial^{k} \rho_{\mathrm{Tot}}\right) \Delta \Psi-2 \nabla\left(\partial^{k} \rho_{\mathrm{Tot}}\right) \cdot \nabla \Psi\right] \\
& \quad \times\left[-b^{2} \chi \Delta \tilde{\rho}^{(k)}-b^{2} \nabla \chi \cdot \nabla \tilde{\rho}^{(k)}+(p-1) \chi \rho_{D}^{p-2} \rho_{\mathrm{Tot}} \tilde{\rho}^{(k)}\right]
\end{aligned}
$$




$$
\begin{aligned}
& -\int k \partial \rho_{\mathrm{Tot}} \partial^{k-1} \Delta \Psi\left[-b^{2} \chi \Delta \tilde{\rho}^{(k)}-b^{2} \nabla \chi \cdot \nabla \tilde{\rho}^{(k)}\right. \\
& \left.+(p-1) \chi \rho_{D}^{p-2} \rho_{\mathrm{Tot}} \tilde{\rho}^{(k)}\right]-\int\left(\rho_{\mathrm{Tot}} \Delta \Psi^{(k)}+2 \nabla \rho_{\mathrm{Tot}} \cdot \nabla \Psi^{(k)}\right) \\
\times & {\left[-b^{2} \chi \Delta \tilde{\rho}^{(k)}-b^{2} \nabla \chi \cdot \nabla \tilde{\rho}^{(k)}+(p-1) \chi \rho_{D}^{p-2} \rho_{\mathrm{Tot}} \tilde{\rho}^{(k)}\right] } \\
= & b^{2} \int \chi \nabla F_{1} \cdot \nabla \tilde{\rho}^{(k)}+(p-1) \int \chi F_{1} \rho_{D}^{p-2} \rho_{\mathrm{Tot}} \tilde{\rho}^{(k)} \\
+ & \int\left[\left(H_{1}-k H_{2}\right) \tilde{\rho}^{(k)}-H_{2} \Lambda \tilde{\rho}^{(k)}-\left(\partial^{k} \rho_{\mathrm{Tot}}\right) \Delta \Psi-2 \nabla\left(\partial^{k} \rho_{\mathrm{Tot}}\right) \cdot \nabla \Psi\right] \\
\times & {\left[-b^{2} \nabla \cdot\left(\chi \nabla \tilde{\rho}^{(k)}\right)+(p-1) \chi \rho_{D}^{p-2} \rho_{\mathrm{Tot}} \tilde{\rho}^{(k)}\right] } \\
& -\int k \partial \rho_{\mathrm{Tot}} \partial^{k-1} \Delta \Psi\left[-b^{2} \nabla \cdot\left(\chi \nabla \tilde{\rho}^{(k)}\right)+(p-1) \chi \rho_{D}^{p-2} \rho_{\mathrm{Tot}} \tilde{\rho}^{(k)}\right] \\
+ & b^{2} \int \nabla \chi \cdot \nabla \tilde{\rho}^{(k)}\left(\rho_{\mathrm{Tot}} \Delta \Psi^{(k)}+2 \nabla \rho_{\mathrm{Tot}} \cdot \nabla \Psi^{(k)}\right) \\
& -\int \chi\left(\rho_{\mathrm{Tot}} \Delta \Psi^{(k)}+2 \nabla \rho_{\mathrm{Tot}} \cdot \nabla \Psi^{(k)}\right) \\
& \times\left[-b^{2} \Delta \tilde{\rho}^{(k)}+(p-1) \rho_{D}^{p-2} \rho_{\mathrm{Tot}} \tilde{\rho}^{(k)}\right] .
\end{aligned}
$$

Similarly:

$$
\begin{aligned}
- & \int \partial_{\tau} \Psi^{(k)}\left[2 \chi \rho_{\mathrm{Tot}} \nabla \rho_{\mathrm{Tot}} \cdot \nabla \Psi^{(k)}+\chi \rho_{\mathrm{Tot}}^{2} \Delta \Psi^{(k)}+\rho_{\mathrm{Tot}}^{2} \nabla \chi \cdot \nabla \Psi^{(k)}\right] \\
= & -\int F_{2} \nabla \cdot\left(\chi \rho_{\mathrm{Tot}}^{2} \nabla \Psi^{(k)}\right) \\
& -\int\left\{b^{2}\left(\frac{\partial^{k} \Delta \rho_{\mathrm{Tot}}}{\rho_{\mathrm{Tot}}}-\frac{k \partial^{k-1} \Delta \rho_{\mathrm{Tot}} \partial \rho_{\mathrm{Tot}}}{\rho_{\mathrm{Tot}}^{2}}\right)\right\} \\
& \times\left[2 \chi \rho_{\mathrm{Tot}} \nabla \rho_{\mathrm{Tot}} \cdot \nabla \Psi^{(k)}+\chi \rho_{\mathrm{Tot}}^{2} \Delta \Psi^{(k)}+\rho_{\mathrm{Tot}}^{2} \nabla \chi \cdot \nabla \Psi^{(k)}\right] \\
& -\int\left\{-k H_{2} \Psi^{(k)}-H_{2} \Lambda \Psi^{(k)}-\mu(r-2) \Psi^{(k)}-2 \nabla \Psi \cdot \nabla \Psi^{(k)}\right. \\
& \left.-\left[(p-1) \rho_{D}^{p-2} \tilde{\rho}^{(k)}+k(p-1)(p-2) \rho_{D}^{p-3} \partial \rho_{D} \partial^{k-1} \tilde{\rho}\right]\right\} \\
& \times\left[2 \chi \rho_{\mathrm{Tot}} \nabla \rho_{\mathrm{Tot}} \cdot \nabla \Psi^{(k)}+\chi \rho_{\mathrm{Tot}}^{2} \Delta \Psi^{(k)}+\rho_{\mathrm{Tot}}^{2} \nabla \chi \cdot \nabla \Psi^{(k)}\right] \\
= & \int \chi \rho_{T}^{2} \nabla \Psi^{(k)} \cdot \nabla F_{2}-b^{2} \int\left(\partial^{k} \Delta \rho_{D}+\Delta \tilde{\rho}^{(k)}\right)\left[2 \chi \nabla \rho_{\mathrm{Tot}} \cdot \nabla \Psi^{(k)}\right. \\
& \left.+\chi \rho_{\mathrm{Tot}} \Delta \Psi^{(k)}+\rho_{\mathrm{Tot}} \nabla \chi \cdot \nabla \Psi^{(k)}\right] \\
& +b^{2} \int \frac{k \partial^{k-1} \Delta \rho_{\mathrm{Tot}} \partial \rho_{\mathrm{Tot}}\left[2 \chi \nabla \rho_{\mathrm{Tot}} \cdot \nabla \Psi^{(k)}\right.}{\rho_{\mathrm{Tot}}} \\
& \left.+\chi \rho_{\mathrm{Tot}} \Delta \Psi^{(k)}+\rho_{\mathrm{Tot}} \nabla \chi \cdot \nabla \Psi^{(k)}\right] \\
& -\int\left[-k H_{2} \Psi^{(k)}-H_{2} \Lambda \Psi^{(k)}-\mu(r-2) \Psi^{(k)}-2 \nabla \Psi \cdot \nabla \Psi^{(k)}\right] \nabla \\
& +\left(\chi \rho_{\mathrm{Tot}}^{2} \nabla \Psi^{(k)}\right)+\int(p-1) \rho_{D}^{p-2} \tilde{\rho}^{(k)}\left[2 \chi \rho_{\mathrm{Tot}} \nabla \rho_{\mathrm{Tot}} \cdot \nabla \Psi^{(k)}\right. \\
& \left.+\chi \rho_{\mathrm{Tot}}^{2} \Delta \Psi^{(k)}+\rho_{\mathrm{Tot}}^{2} \nabla \chi \cdot \nabla \Psi^{(k)}\right] \\
&
\end{aligned}
$$




$$
\begin{aligned}
& +\int k(p-1)(p-2) \rho_{D}^{p-3} \partial \rho_{D} \partial^{k-1} \tilde{\rho} \nabla \cdot\left(\chi \rho_{\mathrm{Tot}}^{2} \nabla \Psi^{(k)}\right) \\
& =\int \chi \rho_{T}^{2} \nabla \Psi^{(k)} \cdot \nabla F_{2}-b^{2} \int\left(\partial^{k} \Delta \rho_{D}\right) \nabla \cdot\left(\chi \rho_{\mathrm{Tot}}^{2} \nabla \Psi^{(k)}\right) \\
& +\int\left(-b^{2} \Delta \tilde{\rho}^{(k)}+(p-1) \rho_{D}^{p-2} \rho_{\mathrm{Tot}} \tilde{\rho}^{(k)}\right) \\
& +\left[2 \chi \nabla \rho_{\mathrm{Tot}} \cdot \nabla \Psi^{(k)}+\chi \rho_{\mathrm{Tot}} \Delta \Psi^{(k)}+\rho_{\mathrm{Tot}} \nabla \chi \cdot \nabla \Psi^{(k)}\right] \\
& +b^{2} \int \frac{k \partial^{k-1} \Delta \rho_{\mathrm{Tot}} \partial \rho_{\mathrm{Tot}}}{\rho_{T}^{2}} \nabla \cdot\left(\chi \rho_{\mathrm{Tot}}^{2} \nabla \Psi^{(k)}\right) \\
& -\int\left[-k H_{2} \Psi^{(k)}-H_{2} \Lambda \Psi^{(k)}-\mu(r-2) \Psi^{(k)}-2 \nabla \Psi \cdot \nabla \Psi^{(k)}\right] \nabla \cdot\left(\chi \rho_{\mathrm{Tot}}^{2} \nabla \Psi^{(k)}\right) \\
& +\int k(p-1)(p-2) \rho_{D}^{p-3} \partial \rho_{D} \partial^{k-1} \tilde{\rho} \nabla \\
& +\left(\chi \rho_{\mathrm{Tot}}^{2} \nabla \Psi^{(k)}\right) .
\end{aligned}
$$

This yields the algebraic energy identity:

$$
\begin{aligned}
& \frac{1}{2} \frac{d}{d \tau}\left\{\int b^{2} \chi\left|\nabla \tilde{\rho}^{(k)}\right|^{2}+(p-1) \int \chi \rho_{D}^{p-2} \rho_{\mathrm{Tot}}\left(\tilde{\rho}^{(k)}\right)^{2}+\int \chi \rho_{\mathrm{Tot}}^{2}\left|\nabla \Psi^{(k)}\right|^{2}\right\} \\
& =\frac{1}{2} \int \partial_{\tau} \chi\left\{b^{2}\left|\nabla \tilde{\rho}^{(k)}\right|^{2}+(p-1) \rho_{D}^{p-2} \rho_{\mathrm{Tot}}\left(\tilde{\rho}^{(k)}\right)^{2}+\rho_{\mathrm{Tot}}^{2}\left|\nabla \Psi^{(k)}\right|^{2}\right\} \\
& -b^{2} \int\left(\partial^{k} \Delta \rho_{D}\right) \nabla \cdot\left(\chi \rho_{\text {Tot }}^{2} \nabla \Psi^{(k)}\right) \\
& -e b^{2} \int \chi\left|\nabla \tilde{\rho}^{(k)}\right|^{2}+\int \chi \frac{\partial_{\tau} \rho_{\mathrm{Tot}}}{\rho_{\mathrm{Tot}}}\left[\frac{p-1}{2} \rho_{D}^{p-2} \rho_{\mathrm{Tot}}\left(\tilde{\rho}^{(k)}\right)^{2}+\rho_{T}^{2}\left|\nabla \Psi^{(k)}\right|^{2}\right] \\
& +\frac{p-1}{2} \int \chi(p-2) \frac{\partial_{\tau} \rho_{D}}{\rho_{D}} \rho_{D}^{p-2} \rho_{\operatorname{Tot}}\left(\tilde{\rho}^{(k)}\right)^{2}+\int F_{1} \chi(p-1) \rho_{D}^{p-2} \rho_{\operatorname{Tot}} \tilde{\rho}^{(k)} \\
& +b^{2} \int \chi \nabla F_{1} \cdot \nabla \tilde{\rho}^{(k)}+\int \chi \rho_{T}^{2} \nabla F_{2} \cdot \nabla \Psi^{(k)} \\
& +\int\left[\left(H_{1}-k H_{2}\right) \tilde{\rho}^{(k)}-H_{2} \Lambda \tilde{\rho}^{(k)}-\left(\partial^{k} \rho_{\text {Tot }}\right) \Delta \Psi-2 \nabla\left(\partial^{k} \rho_{\text {Tot }}\right) \cdot \nabla \Psi\right] \\
& \times\left[-b^{2} \nabla \cdot\left(\chi \nabla \tilde{\rho}^{(k)}\right)+(p-1) \chi \rho_{D}^{p-2} \rho_{\operatorname{Tot}} \tilde{\rho}^{(k)}\right] \\
& -\int\left[-k H_{2} \Psi^{(k)}-H_{2} \Lambda \Psi^{(k)}-\mu(r-2) \Psi^{(k)}-2 \nabla \Psi \cdot \nabla \Psi^{(k)}\right] \\
& \times \nabla \cdot\left(\chi \rho_{\mathrm{Tot}}^{2} \nabla \Psi^{(k)}\right) \\
& -\int k \partial \rho_{\operatorname{Tot}} \partial^{k-1} \Delta \Psi\left[-b^{2} \nabla \cdot\left(\chi \nabla \tilde{\rho}^{(k)}\right)+(p-1) \chi \rho_{D}^{p-2} \rho_{\text {Tot }} \tilde{\rho}^{(k)}\right] \\
& +b^{2} \int \frac{k \partial^{k-1} \Delta \rho_{\mathrm{Tot}} \partial \rho_{\mathrm{Tot}}}{\rho_{T}^{2}} \nabla \cdot\left(\chi \rho_{\mathrm{Tot}}^{2} \nabla \Psi^{(k)}\right) \\
& +\int k(p-1)(p-2) \rho_{D}^{p-3} \partial \rho_{D} \partial^{k-1} \tilde{\rho} \nabla \cdot\left(\chi \rho_{\text {Tot }}^{2} \nabla \Psi^{(k)}\right) \\
& +b^{2} \int \nabla \chi \cdot \nabla \tilde{\rho}^{(k)}\left(\rho_{\mathrm{Tot}} \Delta \Psi^{(k)}+2 \nabla \rho_{\mathrm{Tot}} \cdot \nabla \Psi^{(k)}\right) \\
& +\int\left(-b^{2} \Delta \tilde{\rho}^{(k)}+(p-1) \rho_{D}^{p-2} \rho_{\operatorname{Tot}} \tilde{\rho}^{(k)}\right)\left[\rho_{\mathrm{Tot}} \nabla \chi \cdot \nabla \Psi^{(k)}\right] .
\end{aligned}
$$




\subsection{Weighted $L^{2}$ bound for $m \leq k_{m}-1$}

Given $\sigma \in \mathbb{R}$, we recall the notation

$$
\mid \begin{aligned}
& \|\tilde{\rho}, \Psi\|_{k, \sigma}^{2}=\sum_{m=0}^{k} \int \chi_{k, m, \sigma}\left[b^{2}\left|\nabla \tilde{\rho}_{m}\right|^{2}+(p-1) \rho_{D}^{p-2} \rho_{\text {Tot }} \tilde{\rho}_{m}^{2}\right. \\
& \left.\quad+\rho_{\text {Tot }}^{2}\left|\nabla \Psi_{m}\right|^{2}\right], \\
& \chi_{k, m, \sigma}(Z)=\frac{1}{\langle Z\rangle^{2(k-m+\sigma)}} \xi_{k}\left(\frac{Z}{Z^{*}}\right) .
\end{aligned}
$$

We let

$$
\begin{aligned}
I_{k, \sigma}= & \int \frac{\xi_{k}\left(\frac{Z}{Z^{*}}\right)}{\langle Z\rangle^{2 \sigma}}\left[b^{2}\left|\nabla \tilde{\rho}^{(k)}\right|^{2}+(p-1) \rho_{D}^{p-2} \rho_{\mathrm{Tot}}\left(\tilde{\rho}^{(k)}\right)^{2}\right. \\
& \left.+\rho_{\mathrm{Tot}}^{2}\left|\nabla \Psi^{(k)}\right|^{2}\right] .
\end{aligned}
$$

Lemma 5.2 (Weighted $L^{2}$ bound) Recall the definition (4.28), (4.29) of $\sigma(m)$ and let

$$
\mid \begin{aligned}
& \sigma=\sigma(k) \\
& v+\frac{2(r-1)}{p-1}=\tilde{v}
\end{aligned}
$$

then there exists $c_{k_{m}}>0$ such that for all $0<\tilde{v}<\tilde{v}\left(k_{m}\right) \ll 1$ and $b_{0}<$ $b_{0}\left(k_{m}\right) \ll 1$, for all $1 \leq k \leq k_{m}-1, I_{k}:=I_{k, \sigma(k)}$ given by (5.11) satisfies the differential inequality

$$
\frac{d I_{k}}{d \tau}+2 \mu \tilde{\nu} I_{k} \leq e^{-c_{k_{m}} \tau}
$$

We claim that Lemma 5.2 implies Proposition 5.1.

Proof of Proposition 5.1 Integrating (5.13) on the interval $\left[\tau_{0}, \tau\right]$, with initial data prescribed at $\tau_{0}$, we obtain

$$
I_{k}(\tau) \leq e^{-2 \mu \tilde{\nu}\left(\tau-\tau_{0}\right)} I_{k}\left(\tau_{0}\right)+\frac{1}{c_{k_{m}}-2 \mu \tilde{\nu}}\left(e^{-2 \mu \tilde{\nu}\left(\tau-\tau_{0}\right)-c_{k_{m}} \tau_{0}}-e^{-c_{k_{m}} \tau}\right) .
$$

We now recall, see Remark 4.3, that $I_{k}\left(\tau_{0}\right) \leq e^{-c \tau_{0}}$. Choosing $4 \mu \tilde{\nu} \leq$ $\min \left\{c, c_{k_{m}}\right\}$ we obtain that

$$
I_{k}(\tau) \leq 2 e^{-2 \mu \tilde{\nu} \tau}
$$


We now recall from (4.35) and (4.36) for even $m$ that $\|\tilde{\rho}, \Psi\|_{m, \sigma}$ controls all the corresponding Sobolev norms: let a multi-index $\alpha=\left(\alpha_{1}, \ldots, \alpha_{d}\right)$ with

$$
\alpha_{1}+\cdots+\alpha_{d}=|\alpha|, \quad \nabla^{\alpha}:=\partial_{1}^{\alpha_{1}} \cdots \partial_{d}^{\alpha_{d}},
$$

then for all $|\alpha|=k, \quad 0 \leq k \leq m$,

$$
\begin{aligned}
& b^{2} \int \chi_{k, m, \sigma}\left|\nabla \nabla^{\alpha} \tilde{\rho}\right|^{2}+(p-1) \int \chi_{k, m, \sigma} \rho_{D}^{p-2} \rho_{\mathrm{Tot}}\left|\nabla^{\alpha} \tilde{\rho}\right|^{2} \\
& \quad+\int \chi_{k, m . \sigma} \rho_{\mathrm{Tot}}^{2}\left|\nabla \nabla^{\alpha} \Psi\right|^{2}, \\
& \quad \lesssim\|\tilde{\rho}, \Psi\|_{k, \sigma}^{2},
\end{aligned}
$$

and similarly the norm $\|\tilde{\rho}, \Psi\|_{k, \sigma}^{2}$ (with even $k$ ) is equivalent to the one where $\partial^{m}$ with $1 \leq m \leq k$ derivatives are replaced by $\Delta^{m}$ with $1 \leq m \leq \frac{k}{2}$.

We now claim

$$
\|\tilde{\rho}, \Psi\|_{m, \sigma(m)}^{2} \leq \sum_{k=0}^{m} I_{k, \sigma(k)} .
$$

Combining this with (5.14) concludes the proof of (5.1) (with $\left.c_{k_{m}}^{*}=\mu \tilde{v}\right)$. Proof of (5.16). Indeed,

$$
\begin{aligned}
\|\tilde{\rho}, \Psi\|_{m, \sigma(m)}^{2}= & \sum_{k=0}^{m} \int \chi_{m, k, \sigma(m)} \\
& {\left[b^{2}\left|\nabla \tilde{\rho}^{(k)}\right|^{2}+(p-1) \rho_{D}^{p-2} \rho_{\mathrm{Tot}}\left(\tilde{\rho}^{(k)}\right)^{2}+\rho_{\mathrm{Tot}}^{2}\left|\nabla \Psi^{(k)}\right|^{2}\right] } \\
= & \sum_{k=0}^{m} \int \frac{\langle Z\rangle^{2 k}}{\langle Z\rangle^{2(m+\sigma(m))}} \xi_{m}(x) \\
& {\left[b^{2}\left|\nabla \tilde{\rho}^{(k)}\right|^{2}+(p-1) \rho_{D}^{p-2} \rho_{\mathrm{Tot}}\left(\tilde{\rho}^{(k)}\right)^{2}+\rho_{\mathrm{Tot}}^{2}\left|\nabla \Psi^{(k)}\right|^{2}\right] }
\end{aligned}
$$

and

$$
\begin{aligned}
\sum_{k=0}^{m} I_{k, \sigma(k)}= & \sum_{k=0}^{m} \int \frac{\xi_{k}(x)}{\langle Z\rangle^{2 \sigma(k)}} \\
& {\left[b^{2}\left|\nabla \tilde{\rho}^{(k)}\right|^{2}+(p-1) \rho_{D}^{p-2} \rho_{\mathrm{Tot}}\left(\tilde{\rho}^{(k)}\right)^{2}+\rho_{\mathrm{Tot}}^{2}\left|\nabla \Psi^{(k)}\right|^{2}\right] } \\
= & \sum_{k=0}^{m} \int \frac{\langle Z\rangle^{2 k} \xi_{k}(x)}{\langle Z\rangle^{2(\sigma(k)+k)}}
\end{aligned}
$$




$$
\left[b^{2}\left|\nabla \tilde{\rho}^{(k)}\right|^{2}+(p-1) \rho_{D}^{p-2} \rho_{\operatorname{Tot}}\left(\tilde{\rho}^{(k)}\right)^{2}+\rho_{\text {Tot }}^{2}\left|\nabla \Psi^{(k)}\right|^{2}\right]
$$

and hence (5.16) follows from $\sigma(k)+k \leq \sigma(m)+m$ and $\xi_{k}(x) \geq \xi_{m}(x)$ for $0 \leq k \leq m$.

\subsection{Proof of Lemma 5.2}

This follows from the energy identity (5.10) coupled with the pointwise bound (4.40) to control the nonlinear term.

Step 1 Interpolation bounds. In what follows we use the convention $\lesssim$ to denote any dependence on the universal constants, including $k_{m}$. Constants $c, c_{k_{m}}$ will stand for generic, universal small constants.

Our main technical tool below will be the following interpolation bound: for any $0 \leq m \leq k_{m}-1$ and $\delta>0$, there exists $c_{\delta, k_{m}}>0$ such that

$$
\|\tilde{\rho}, \Psi\|_{m, \sigma(m)+\delta}^{2} \leq e^{-c_{\delta, k_{m}} \tau} .
$$

Indeed, the claim follows by interpolating the local decay bootstrap bound (4.39) and the bound (4.38) for the highest Sobolev norm for $Z \leq Z_{c}^{*}:=\left(Z^{*}\right)^{c}$ and using the global weighted Sobolev bound for (4.34) for $Z \geq Z_{c}^{*}$

$$
\begin{aligned}
\|\tilde{\rho}, \Psi\|_{m, \sigma(m)+\delta}^{2} & \leq\left(Z_{c}^{*}\right)^{C_{k_{m}}} e^{-c_{k_{m}} \tau}+\frac{1}{\left(Z_{c}^{*}\right)^{2 \delta}}\|\tilde{\rho}, \Psi\|_{m, \sigma(m)}^{2} \\
& \leq e^{-c_{\delta, k_{m}} \tau}
\end{aligned}
$$

Above, on the set $Z \leq Z_{c}^{*}$, we can replace the norm $\|\tilde{\rho}, \Psi\|_{m, \sigma(m)+\delta}^{2}$ by $\left(Z_{c}^{*}\right) C_{k_{m}}$ with some large constant $C_{k_{m}}$, times the unweighted Sobolev norm $\|\tilde{\rho}, \Psi\|_{H^{m}\left(Z \leq Z_{c}^{*}\right)}^{2}$ and then interpolate the latter between the Sobolev bounds (4.39) and (4.38). That will bring an additional factor $\left(Z_{c}^{*}\right)^{C}$, which can be absorbed by $C_{k_{m}}$, and the decaying factor $e^{-c_{k_{m}} \tau}$ with a small constant $c_{k_{m}}$, explicitly dependent on $k_{m}$ and $\delta$. We can then choose $c$ small enough (dependent on $C_{k_{m}}$ ) to obtain the second inequality in (5.18).

We will also use the bound for the damped profile from (4.7), (4.8) and (4.9):

$$
\left|Z^{k} \partial_{Z}^{k} \rho_{D}\right| \lesssim \frac{1}{\langle Z\rangle^{\frac{2(r-1)}{p-1}}} \mathbf{1}_{Z \leq Z^{*}}+\frac{1}{\left(Z^{*}\right)^{\frac{2(r-1)}{p-1}}} \frac{1}{\left(\frac{Z}{Z^{*}}\right)^{n_{P}}} \mathbf{1}_{Z \geq Z^{*}}
$$

We will also use the bound

$$
\chi_{k-1, k-1, \sigma(k-1)} \leq\langle Z\rangle^{2(\alpha+\beta)} \chi_{k, k, \sigma(k)} \leq\langle Z\rangle^{2} \chi_{k, k, \sigma(k)},
$$


which follows from

$$
\sigma(k-1)+\alpha \geq \sigma(k), \quad \tilde{\sigma}(k-1) \leq \tilde{\sigma}(k)+\beta
$$

and $\alpha+\beta \leq 1$.

Step 2 Energy identity. We run (5.10) with

$$
\chi=\frac{1}{\langle Z\rangle^{2 \sigma}} \xi_{k}\left(\frac{Z}{Z^{*}}\right), \quad \sigma=\sigma(k), \quad 1 \leq k \leq k_{m}-1
$$

with $\xi_{k}(x)=1$ for $x \leq 1$ and $\xi_{k}(x)=x^{2 \tilde{\sigma}(k)}$ for $x>1$, and estimate all terms. In our notations

$$
\chi=\chi_{k, k, \sigma(k)}
$$

From (4.28), (4.29) and recalling $m_{0}=\frac{4 k_{m}}{9}+1$ :

$$
\begin{aligned}
\sigma(k)+k & =\mid \begin{array}{l}
\sigma_{v} \text { for } 0 \leq k \leq m_{0} \\
-\alpha\left(k_{m}-k\right)+k=(\alpha+1)\left(k-m_{0}\right)+\sigma_{v} \text { for } m_{0} \leq k \leq k_{m}
\end{array} \\
& \geq \sigma_{v}
\end{aligned}
$$

and

$$
\begin{aligned}
\tilde{\sigma}(k) & =\mid \begin{array}{l}
n_{P}-\frac{2(r-1)}{p-1}-(r-2)+2 \tilde{v} \text { for } 0 \leq k \leq \frac{2 k_{m}}{3}+1, \\
\beta\left(k_{m}-k\right) \text { for } \frac{2 k_{m}}{3}+1 \leq k \leq k_{m},
\end{array} \\
\leq & n_{P}-\frac{2(r-1)}{p-1}-(r-2)+2 \tilde{v},
\end{aligned}
$$

which implies

$$
\begin{aligned}
\chi & =\frac{1}{\langle Z\rangle^{2 \sigma(k)}} \xi_{k}\left(\frac{Z}{Z^{*}}\right) \\
& \lesssim \frac{1}{\langle Z\rangle^{2 \sigma(k)}}\left[1+\left(\frac{Z}{Z^{*}}\right)^{2 n_{P}-\frac{4(r-1)}{p-1}-2(r-2)+4 \tilde{v}} \mathbf{1}_{Z \geq Z^{*}}\right] \\
& \lesssim \frac{1}{\langle Z\rangle^{-2 k+2\left(\frac{d}{2}+\tilde{v}-\frac{2(r-1)}{p-1}-(r-1)\right)}}+\frac{\left(\frac{Z}{Z^{*}}\right)^{2 n_{P}-\frac{4(r-1)}{p-1}-2(r-2)+4 \tilde{v}}}{\langle Z\rangle^{-2 k+2\left(\frac{d}{2}+\tilde{v}-\frac{2(r-1)}{p-1}-(r-1)\right)}} \mathbf{1}_{Z \geq Z^{*}} .
\end{aligned}
$$


which we will use below. The following additional inequality will be of particular significance $\left(b=\left(Z^{*}\right)^{2-r}\right)$ :

$$
\begin{aligned}
\rho_{D}^{2} \chi & \lesssim \frac{1}{\langle Z\rangle^{-2 k+2\left(\frac{d}{2}+\tilde{v}-(r-1)\right)}}\left[\mathbf{1}_{Z \leq Z^{*}}+\left(\frac{Z}{Z^{*}}\right)^{4 \tilde{v}-2(r-2)} \mathbf{1}_{Z \geq Z^{*}}\right] \\
& =\frac{1}{\langle Z\rangle^{-2 k+2\left(\frac{d}{2}+\tilde{v}-(r-1)\right)}} \mathbf{1}_{Z \leq Z^{*}}+\frac{1}{b^{2-\frac{4 \tilde{v}}{r-2}}\langle Z\rangle^{-2 k+2\left(\frac{d}{2}-\tilde{v}-1\right)}} \mathbf{1}_{Z \geq Z^{*}}
\end{aligned}
$$

Step 3 Leading order terms. In what follows, we will systematically use the standard Pohozhaev identity:

$$
\begin{aligned}
\int \Delta g F \cdot \nabla g d x & =\sum_{i, j=1}^{d} \int \partial_{i}^{2} g F_{j} \partial_{j} g d x=-\sum_{i, j=1}^{d} \int \partial_{i} g\left(\partial_{i} F_{j} \partial_{j} g+F_{j} \partial_{i, j}^{2} g\right) \\
& =-\sum_{i, j=1}^{d} \int \partial_{i} F_{j} \partial_{i} g \partial_{j} g+\frac{1}{2} \int|\nabla g|^{2} \nabla \cdot F
\end{aligned}
$$

which becomes in the case of spherically symmetric functions

$$
\begin{aligned}
\int_{\mathbb{R}^{d}} f \Delta g \partial_{r} g d x & =c_{d} \int_{\mathbb{R}^{+}} \frac{f}{r^{d-1}} \partial_{r}\left(r^{d-1} \partial_{r} g\right) r^{d-1} \partial_{r} g d r \\
& =-\frac{1}{2} \int_{\mathbb{R}^{d}}\left|\partial_{r} g\right|^{2}\left[f^{\prime}-\frac{d-1}{r} f\right] d x,
\end{aligned}
$$

Cross terms. We consider

$$
A_{1}=b^{2} k\left[\int \partial \rho_{\mathrm{Tot}} \partial^{k-1} \Delta \Psi \nabla \cdot\left(\chi \nabla \tilde{\rho}^{(k)}\right)+\frac{\partial^{k-1} \Delta \rho_{\mathrm{Tot}} \partial \rho \rho_{\mathrm{Tot}}}{\rho_{T}^{2}} \nabla \cdot\left(\chi \rho_{\mathrm{Tot}}^{2} \nabla \Psi^{(k)}\right)\right] .
$$

We compute

$$
\begin{aligned}
\partial \rho_{\mathrm{Tot}} \partial^{k-1} \Delta \Psi \nabla \cdot\left(\chi \nabla \tilde{\rho}^{(k)}\right)+\frac{\partial^{k-1} \Delta \rho_{\mathrm{Tot}} \partial \rho_{\mathrm{Tot}}}{\rho_{T}^{2}} \nabla \cdot\left(\chi \rho_{\mathrm{Tot}}^{2} \nabla \Psi^{(k)}\right) \\
=\partial \rho_{\operatorname{Tot}} \partial^{k-1} \Delta \Psi\left[\nabla \chi \cdot \nabla \tilde{\rho}^{(k)}+\chi \Delta \tilde{\rho}^{(k)}\right] \\
\quad+\partial^{k-1} \Delta \rho_{\operatorname{Tot}} \partial \rho_{\operatorname{Tot}}\left[\nabla \chi \cdot \nabla \Psi^{(k)}\right. \\
\left.\quad+2 \chi \frac{\nabla \rho_{\mathrm{Tot}}}{\rho_{\mathrm{Tot}}} \cdot \nabla \Psi^{(k)}+\chi \Delta \Psi^{(k)}\right] \\
=\partial \rho_{\operatorname{Tot}} \partial^{k-1} \Delta \Psi \nabla \chi \cdot \nabla \tilde{\rho}^{(k)}+\partial^{k-1} \Delta \rho_{\operatorname{Tot}} \partial \rho_{\operatorname{Tot}} \nabla \chi \cdot \nabla \Psi^{(k)}
\end{aligned}
$$




$$
\begin{aligned}
& +2 \partial^{k-1} \Delta \rho_{\operatorname{Tot}} \partial \rho_{\operatorname{Tot}} \chi \frac{\nabla \rho \operatorname{Tot}}{\rho_{\text {Tot }}} \cdot \nabla \Psi^{(k)} \\
& +\chi \partial \rho_{\operatorname{Tot}} \partial^{k-1} \Delta \Psi \Delta \tilde{\rho}^{(k)}+\chi \partial^{k-1} \Delta \rho_{\operatorname{Tot}} \partial \rho \operatorname{Tot} \Delta \Psi^{(k)} .
\end{aligned}
$$

The last 2 terms require an integration by parts:

$$
\begin{aligned}
& b^{2} k\left|\int\left[\chi \partial \rho_{\operatorname{Tot}} \partial^{k-1} \Delta \Psi \Delta \tilde{\rho}^{(k)}+\chi \partial^{k-1} \Delta \rho_{\operatorname{Tot}} \partial \rho_{\mathrm{Tot}} \Delta \Psi^{(k)}\right]\right| \\
&=b^{2} k\left|\int\left[-\left(\Delta \partial^{k-1} \tilde{\rho}\right) \partial\left(\chi \partial \rho_{\operatorname{Tot}} \partial^{k-1} \Delta \Psi\right)+\chi \partial^{k-1} \Delta \rho_{\operatorname{Tot}} \partial \rho_{\operatorname{Tot}} \Delta \Psi^{(k)}\right]\right| \\
&=b^{2} k \mid \int\left[-\partial^{k-1} \Delta \tilde{\rho}\left[\chi \partial^{2} \rho_{\operatorname{Tot}} \partial^{k-1} \Delta \Psi+\partial \chi \partial \rho_{\operatorname{Tot}} \partial^{k-1} \Delta \Psi\right]\right. \\
&\left.\quad+\chi \partial^{k-1} \Delta \rho_{D} \partial \rho_{\operatorname{Tot}} \Delta \Psi^{(k)}\right] \mid \\
& \lesssim C_{k} b^{2} \int \chi\left|\partial^{k-1} \Delta \Psi\right|\left[\left|\partial^{k-1} \Delta \tilde{\rho} \partial^{2} \rho_{\mathrm{Tot}}\right|\right. \\
&\left.+\frac{\left|\partial^{k-1} \Delta \tilde{\rho} \partial \rho_{\mathrm{Tot}}\right|}{\langle Z\rangle}+\left|\partial\left(\partial^{k-1} \Delta \rho_{D} \partial \rho_{\mathrm{Tot}}\right)\right|\right] \\
& \lesssim C_{k} b^{2} \int \chi \rho \operatorname{Tot}\left|\partial^{k-1} \Delta \Psi\right|\left[\frac{\rho_{D}}{\langle Z\rangle^{k+2}}+\frac{\left|\partial^{k-1} \Delta \tilde{\rho}\right|}{\langle Z\rangle}\right] \\
& \lesssim \sum_{|\alpha|=k} \int \frac{\chi \rho_{\mathrm{Tot}}^{2}}{\langle Z\rangle}\left|\nabla \partial^{\alpha} \Psi\right|^{2}+c_{k} b^{4} \int \frac{\chi}{\langle Z\rangle}\left|\nabla \partial^{\alpha} \tilde{\rho}\right|^{2}+b^{4} \int \chi \frac{\rho_{D}^{2}}{\langle Z\rangle^{2 k+3}},
\end{aligned}
$$

where in penultimate inequality we used the pointwise bound (4.40).

We now estimate the source term from (5.26):

$$
\begin{aligned}
b^{4} \int \chi \frac{\rho_{D}^{2}}{\langle Z\rangle^{2 k+3} \lesssim} b^{4} \int_{Z \leq Z^{*}} \frac{Z^{d-1} d Z}{\langle Z\rangle^{2 k+3-2 k+2\left(\frac{d}{2}+\tilde{v}-(r-1)\right)}} \\
\quad+b^{2} \int_{Z \geq Z^{*}}\left(\frac{Z}{Z^{*}}\right)^{4 \tilde{v}} \frac{Z^{d-1} d Z}{\langle Z\rangle^{2 k+3-2 k+2\left(\frac{d}{2}+\tilde{v}-1\right)}} \\
\lesssim b^{4} \int_{Z \leq Z^{*}}\langle Z\rangle^{2(r-2)-2-2 \tilde{v}} d Z \\
\quad+b^{2} \int_{Z \geq Z^{*}}\left(\frac{Z}{Z^{*}}\right)^{4 \tilde{v}}\langle Z\rangle^{-2-2 \tilde{v}} d Z \\
\lesssim b^{4}\left(Z^{*}\right)^{2(r-2)-1-2 \tilde{v}} \lesssim e^{-c \tau}
\end{aligned}
$$


and hence, using (5.18),

$$
\begin{aligned}
& b^{2} k\left|\int\left[\chi \partial \rho_{\operatorname{Tot}} \partial^{k-1} \Delta \Psi \Delta \tilde{\rho}^{(k)}+\chi \partial^{k-1} \Delta \rho_{\operatorname{Tot}} \partial \rho_{\operatorname{Tot}} \Delta \Psi^{(k)}\right]\right| \\
& \quad \lesssim e^{-c \tau}+\|\tilde{\rho}, \Psi\|_{k, \sigma+\frac{1}{2}}^{2} \lesssim e^{-c_{k_{m}} \tau} .
\end{aligned}
$$

We estimate similarly,

$$
\begin{aligned}
& k b^{2} \mid \partial \rho_{\mathrm{Tot}} \partial^{k-1} \Delta \Psi \nabla \chi \cdot \nabla \tilde{\rho}^{(k)}+\partial^{k-1} \Delta \rho_{\mathrm{Tot}} \partial \rho_{\mathrm{Tot}} \nabla \chi \cdot \nabla \Psi^{(k)} \\
& \quad+2 \partial^{k-1} \Delta \rho_{\mathrm{Tot}} \partial \rho_{\mathrm{Tot}} \chi \frac{\nabla \rho_{\mathrm{Tot}}}{\rho_{\mathrm{Tot}}} \cdot \nabla \Psi^{(k)} \mid \\
& \lesssim \sum_{|\alpha|=k}\left[b^{4} \int \frac{\chi}{\langle Z\rangle}\left|\nabla \partial^{\alpha} \tilde{\rho}\right|^{2}+\int \frac{\chi}{\langle Z\rangle^{3}} \rho_{\mathrm{Tot}}^{2}\left|\nabla \partial^{\alpha} \Psi\right|^{2}\right]+b^{4} \int \chi \frac{\rho_{D}^{2}}{\langle Z\rangle^{2 k+3}} \\
& \lesssim e^{-c_{k_{m}} \tau}+\|\tilde{\rho}, \Psi\|_{k, \sigma+\frac{1}{2}}^{2} \lesssim e^{-c_{k_{m}} \tau} .
\end{aligned}
$$

The remaining cross terms are estimated as follows.

$$
\begin{aligned}
& k(p-1)\left|\int \chi \partial \rho_{\mathrm{Tot}} \partial^{k-1} \Delta \Psi \rho_{D}^{p-2} \rho_{\mathrm{Tot}} \tilde{\rho}^{(k)}\right| \\
& \quad \lesssim c_{k} \int \chi \frac{\rho_{\mathrm{Tot}}^{p-1}}{\langle Z\rangle} \rho_{\mathrm{Tot}}\left|\partial^{k-1} \Delta \Psi \| \tilde{\rho}^{(k)}\right| \\
& \lesssim \int \frac{\chi}{\langle Z\rangle} \rho_{D}^{p-1}\left(\tilde{\rho}^{(k)}\right)^{2}+\int \frac{\chi}{\langle Z\rangle} \rho_{\mathrm{Tot}}^{2}\left|\nabla \partial^{\alpha} \Psi\right|^{2} \\
& \quad \leq\|\tilde{\rho}, \Psi\|_{k, \sigma+\frac{1}{2}}^{2} \lesssim e^{-c_{k m} \tau}
\end{aligned}
$$

where we used that $p \geq 1$ and a trivial bound $\left|\rho_{D}\right| \lesssim 1$. Similarly,

$$
\begin{aligned}
& \int\left|(p-1) \rho_{D}^{p-2} \tilde{\rho}^{(k)} \rho_{T}^{2} \nabla \chi \cdot \nabla \Psi^{(k)}\right| \\
& \quad \lesssim \int \frac{\chi}{\langle Z\rangle} \rho_{D}^{p-1}\left(\tilde{\rho}^{(k)}\right)^{2}+\int \frac{\chi}{\langle Z\rangle} \rho_{\mathrm{Tot}}^{2}\left|\nabla \partial^{\alpha} \Psi\right|^{2} \\
& \quad \leq\|\tilde{\rho}, \Psi\|_{k, \sigma+\frac{1}{2}}^{2} \lesssim e^{-c_{k_{m}} \tau} .
\end{aligned}
$$


The other remaining cross term is estimated using an integration by parts:

$$
\begin{aligned}
& k(p-1)(p-2)\left|\int \nabla \cdot\left(\chi \rho_{\mathrm{Tot}}^{2} \nabla \Psi^{(k)}\right) \rho_{D}^{p-3} \partial \rho_{D} \partial^{k-1} \tilde{\rho}\right| \\
& \quad \lesssim \int \frac{\chi}{\langle Z\rangle} \rho_{D}^{p-1}\left|\nabla \tilde{\rho}_{k-1}\right|^{2}+\int \frac{\chi}{\langle Z\rangle^{3}} \rho_{D}^{p-1} \tilde{\rho}_{k-1}^{2}+\int \frac{\chi}{\langle Z\rangle} \rho_{\mathrm{Tot}}^{2}\left|\nabla \partial^{\alpha} \Psi\right|^{2} \\
& \quad \leq\|\rho, \Psi\|_{k, \sigma+\frac{1}{2}}^{2} \lesssim e^{-c_{k_{m}} \tau} .
\end{aligned}
$$

$\rho_{k}$ terms. We compute using (5.5):

$$
\begin{aligned}
\int & \chi\left(H_{1}-k H_{2}\right) \tilde{\rho}^{(k)}\left(-b^{2} \Delta \tilde{\rho}^{(k)}+(p-1) \rho_{D}^{p-2} \rho_{\mathrm{Tot}} \tilde{\rho}^{(k)}\right) \\
& -b^{2} \int\left[H_{1}-k H_{2}\right] \tilde{\rho}^{(k)} \nabla \chi \cdot \nabla \tilde{\rho}^{(k)} \\
= & \int \chi\left(H_{1}-k H_{2}\right)\left[b^{2}\left|\nabla \tilde{\rho}^{(k)}\right|^{2}+(p-1) \rho_{D}^{p-2} \rho_{\mathrm{Tot}} \tilde{\rho}_{k}^{2}\right] \\
& -\frac{b^{2}}{2} \int\left(\tilde{\rho}^{(k)}\right)^{2} \nabla \cdot\left[\chi \nabla\left(H_{1}-k H_{2}\right)\right] \\
= & -\int \mu \chi\left(k+\frac{2(r-1)}{p-1}+O\left(\frac{1}{\langle Z\rangle^{r}}\right)\right) \\
& \times\left(b^{2}\left|\nabla \tilde{\rho}^{(k)}\right|^{2}+(p-1) \rho_{D}^{p-2} \rho_{\mathrm{Tot}} \tilde{\rho}_{k}^{2}\right) \\
& -\frac{b^{2}}{2} \int\left(\tilde{\rho}^{(k)}\right)^{2} \nabla \cdot\left[\chi \nabla\left(H_{1}-k H_{2}\right)\right] \\
= & O\left(e^{-c_{k m} \tau}\right)-\int \mu \chi\left(k+\frac{2(r-1)}{p-1}\right) \\
& \times\left(b^{2}\left|\nabla \tilde{\rho}^{(k)}\right|^{2}+(p-1) \rho_{D}^{p-2} \rho_{\mathrm{Tot}} \tilde{\rho}_{k}^{2}\right) \\
& -\frac{b^{2}}{2} \int \chi\left(\tilde{\rho}^{(k)}\right)^{2}\left[\frac{\Lambda \chi \Lambda\left(H_{1}-k H_{2}\right)}{\chi Z^{2}}+\Delta\left(H_{1}-k H_{2}\right)\right],
\end{aligned}
$$

where we used the interpolation bound (5.18). Similarly, using that $\chi_{k, k, \sigma}=$ $\langle Z\rangle^{2} \chi_{k, k-1, \sigma}$ and $\left|\rho_{k}\right| \leq\left|\nabla \rho_{k-1}\right|$ as well as (5.5), (5.18) gives

$$
\begin{aligned}
& \frac{b^{2}}{2} \int \chi\left(\tilde{\rho}^{(k)}\right)^{2}\left[\frac{\Lambda \chi \Lambda\left(H_{1}-k H_{2}\right)}{\chi Z^{2}}+\Delta\left(H_{1}-k H_{2}\right)\right] \\
& \lesssim\|\tilde{\rho}, \Psi\|_{k, \sigma(k)+\frac{1}{2}(1+r)}^{2} \lesssim e^{-c_{k m} \tau} .
\end{aligned}
$$


Next using

$$
\left|\partial^{k} \rho_{D}\right| \lesssim \frac{\rho_{D}}{\langle Z\rangle^{k}}
$$

we estimate after an integration by parts:

$$
\begin{aligned}
& b^{2}\left|\int\left(\chi \Delta \tilde{\rho}^{(k)}+\nabla \chi \cdot \nabla \tilde{\rho}^{(k)}\right)\left[\left(\partial^{k} \rho_{D}\right) \Delta \Psi+2 \nabla\left(\partial^{k} \rho_{D}\right) \cdot \nabla \Psi\right]\right| \\
& \lesssim b^{2} \int \chi\left|\nabla \tilde{\rho}^{(k)}\right|\left[\left|\nabla\left(\partial^{k} \rho_{D} \Delta \Psi\right)\right|+\left|\nabla\left(\nabla \partial^{k} \rho_{D} \cdot \nabla \Psi\right)\right|\right. \\
& \left.\quad+\frac{\left|\left(\partial^{k} \rho_{D}\right) \Delta \Psi+2 \nabla\left(\partial^{k} \rho_{D}\right) \cdot \nabla \Psi\right|}{\langle Z\rangle}\right] \\
& \leq b^{2} \int \chi \frac{\left|\nabla \tilde{\rho}^{(k)}\right|^{2}}{\langle Z\rangle}+b^{2} \sum_{j=1}^{3} \int \chi \frac{\langle Z\rangle \rho_{D}^{2}}{\langle Z\rangle^{2 k}}\left(\frac{\left|\partial^{j} \Psi\right|}{\langle Z\rangle^{3-j}}\right)^{2} .
\end{aligned}
$$

We use the pointwise bootstrap bound (4.40)

$$
\left|\langle Z\rangle^{j} \partial^{j} \Psi\right| \leq C_{K}\left[\frac{\mathbf{1}_{Z \leq Z^{*}}}{\langle Z\rangle^{r-2}}+b\right] \lesssim\left[\frac{\mathbf{1}_{Z \leq Z^{*}}}{\langle Z\rangle^{r-2}}+\frac{\mathbf{1}_{Z \geq Z^{*}}}{\left\langle Z^{*}\right\rangle^{r-2}}\right], \quad 1 \leq j \leq 3
$$

to estimate from (5.26):

$$
\begin{aligned}
& b^{2} \sum_{j=1}^{3} \int \chi \frac{\langle Z\rangle \rho_{D}^{2}}{\langle Z\rangle^{2 k}}\left(\frac{\left|\partial^{j} \Psi\right|}{\langle Z\rangle^{3-j}}\right)^{2} \\
& \quad \leq b^{2} C_{K} \int \frac{Z^{d-1} d Z}{\langle Z\rangle^{2\left(\tilde{v}+\frac{d}{2}-(r-1)\right)}} \frac{1}{\langle Z\rangle^{5}}\left(\left[\frac{\mathbf{1}_{Z \leq Z^{*}}}{\langle Z\rangle^{2(r-2)}}+\left(\frac{Z}{Z^{*}}\right)^{4 \tilde{v}} \frac{\mathbf{1}_{Z \geq Z^{*}}}{\langle Z\rangle^{2(r-2)}}\right]\right) \\
& \quad \lesssim e^{-c_{k_{m}} \tau}
\end{aligned}
$$

and hence

$b^{2}\left|\int\left(\chi \Delta \tilde{\rho}^{(k)}+\nabla \chi \cdot \nabla \tilde{\rho}^{(k)}\right)\left[\left(\partial^{k} \rho_{D}\right) \Delta \Psi+2 \nabla\left(\partial^{k} \rho_{D}\right) \cdot \nabla \Psi\right]\right| \lesssim e^{-c_{k_{m}} \tau}$.

For the nonlinear term, we use the Pohozhaev identity (5.27) and the pointwise bound (5.35)

$$
\left|Z^{j} \partial^{j} \Psi\right| \lesssim\langle Z\rangle^{-(r-2)}, \quad j=1, \ldots, 3
$$


to estimate by the interpolation bound (5.18)

$$
\begin{aligned}
& b^{2}\left|\int\left(\chi \Delta \tilde{\rho}^{(k)}+\nabla \chi \cdot \nabla \tilde{\rho}^{(k)}\right)\left[\tilde{\rho}^{(k)} \Delta \Psi+2 \nabla \tilde{\rho}^{(k)} \cdot \nabla \Psi\right]\right| \\
& \quad \lesssim b^{2}\left[\int \chi \frac{\left|\nabla \tilde{\rho}^{(k)}\right|^{2}}{\langle Z\rangle^{r}}+\int \chi \frac{\left(\tilde{\rho}^{(k)}\right)^{2}}{\langle Z\rangle^{r+2}}\right] \\
& \quad \lesssim e^{-c_{k m} \tau} .
\end{aligned}
$$

Note that the last term in the case $k=0$ should be treated with the help of the bound $\tilde{\rho} \lesssim \rho_{D}$ and the estimate (5.28). For $k \neq 0$, we simply use $\left|\rho_{k}\right| \leq\left|\nabla \rho_{k-1}\right|$. We recall that by definition of the norm:

$$
\|\tilde{\rho}, \Psi\|_{k, \sigma}^{2} \gtrsim \sum_{m=0}^{k} \int \chi_{k, k, \sigma} \frac{\rho_{\mathrm{Tot}}^{2}\left|\partial^{m} \nabla \Psi\right|^{2}}{\langle Z\rangle^{2(k-m)}} \gtrsim \sum_{m=1}^{k+1} \int \chi \frac{\rho_{\mathrm{Tot}}^{2}\left|\partial^{m} \Psi\right|^{2}}{\langle Z\rangle^{2(k+1-m)}}
$$

Hence, by the interpolation bound,

$$
\begin{aligned}
& \left|\int \chi\left[\left(\partial^{k} \rho_{D}\right) \Delta \Psi+2 \nabla\left(\partial^{k} \rho_{D}\right) \cdot \nabla \Psi\right](p-1) \rho_{D}^{p-2} \rho_{\mathrm{Tot}} \tilde{\rho}^{(k)}\right| \\
& \quad \lesssim \int \chi \frac{\rho_{D}^{p-2} \rho_{\mathrm{Tot}}\left(\tilde{\rho}^{(k)}\right)^{2}}{\langle Z\rangle}+\int \chi \rho_{\mathrm{Tot}}^{p-2} \rho_{\mathrm{Tot}}^{2}\left[\frac{\left|\partial^{2} \Psi\right|^{2}}{\langle Z\rangle^{2 k-1}}+\frac{|\partial \Psi|^{2}}{\langle Z\rangle^{2(k+1)-1}}\right] \\
& \quad \leq\|\tilde{\rho}, \Psi\|_{k, \sigma+\frac{1}{2}}^{2} \lesssim e^{-c_{k m} \tau} .
\end{aligned}
$$

For the nonlinear term, we integrate by parts and use (5.35):

$$
\begin{aligned}
& \left|\int \chi\left[\tilde{\rho}^{(k)} \Delta \Psi+2 \nabla \tilde{\rho}^{(k)} \cdot \nabla \Psi\right](p-1) \rho_{D}^{p-2} \rho_{\mathrm{Tot}} \tilde{\rho}^{(k)}\right| \\
& \quad \lesssim \int \chi \frac{\rho_{D}^{p-2} \rho_{\mathrm{Tot}}\left(\tilde{\rho}^{(k)}\right)^{2}}{\langle Z\rangle} \lesssim e^{-c_{k_{m}} \tau}
\end{aligned}
$$

From Pohozhaev (5.27) and (5.5):

$$
\begin{aligned}
& -\int H_{2} \chi \Lambda \tilde{\rho}^{(k)}\left(-b^{2} \Delta \tilde{\rho}^{(k)}\right)+b^{2} \int H_{2} \Lambda \rho_{k} \nabla \chi \cdot \nabla \tilde{\rho}^{(k)} \\
& =b^{2}\left[\int \Delta \tilde{\rho}^{(k)}\left(Z \chi H_{2}\right) \cdot \nabla \tilde{\rho}^{(k)}+\int H_{2} \Lambda \rho_{k} \nabla \chi \cdot \nabla \tilde{\rho}^{(k)}\right] \\
& =b^{2}\left[-\sum_{i, j=1}^{d} \int \partial_{i}\left(Z_{j} \chi H_{2}\right) \partial_{i} \tilde{\rho}^{(k)} \partial_{j} \tilde{\rho}^{(k)}+\frac{1}{2} \int\left|\nabla \tilde{\rho}^{(k)}\right|^{2} \nabla \cdot\left(Z \chi H_{2}\right)\right.
\end{aligned}
$$




$$
\begin{aligned}
& \left.+\sum_{i, j=1}^{d} \int H_{2} Z_{j} \partial_{j} \tilde{\rho}^{(k)} \partial_{i} \chi \partial_{i} \tilde{\rho}^{(k)}\right] \\
= & b^{2}\left\{-\sum_{i, j=1}^{d} \int \partial_{i} \tilde{\rho}^{(k)} \partial_{j} \tilde{\rho}^{(k)}\left[\delta_{i j} \chi H_{2}+Z_{j} \partial_{i} \chi H_{2}\right.\right. \\
& \left.+Z_{j} \chi \partial_{i} H_{2}-H_{2} Z_{j} \partial_{i} \chi\right] \\
& \left.+\frac{1}{2} \int\left|\nabla \tilde{\rho}^{(k)}\right|^{2} \chi H_{2}\left[d+\frac{\Lambda \chi}{\chi}+\frac{\Lambda H_{2}}{H_{2}}\right]\right\} \\
= & \frac{\mu}{2} b^{2} \int \chi\left|\nabla \tilde{\rho}^{(k)}\right|^{2}\left[d-2+\frac{\Lambda \chi}{\chi}+O\left(\frac{1}{\langle Z\rangle^{r-1}}\right)\right] .
\end{aligned}
$$

Integrating by parts and using (5.5):

$$
\begin{aligned}
& -\int \chi H_{2} \Lambda \tilde{\rho}^{(k)}\left[(p-1) \rho_{D}^{p-2} \rho_{\mathrm{Tot}} \tilde{\rho}^{(k)}\right] \\
& \quad+\frac{p-1}{2} \int \chi(p-2) \partial_{\tau} \rho_{D} \rho_{D}^{p-3} \rho_{\operatorname{Tot}}\left(\tilde{\rho}^{(k)}\right)^{2} \\
& \quad+\frac{p-1}{2} \int \chi \partial_{\tau} \rho_{\operatorname{Tot}} \rho_{D}^{p-2}\left(\tilde{\rho}^{(k)}\right)^{2} \\
& =\frac{p-1}{2} \int\left(\tilde{\rho}^{(k)}\right)^{2}\left[\nabla \cdot\left(Z \chi H_{2} \rho_{D}^{p-2} \rho_{\operatorname{Tot}}\right)\right. \\
& \left.\quad+\chi \rho_{\operatorname{Tot}} \partial_{\tau}\left(\rho_{D}^{p-2}\right)+\chi \partial_{\tau} \rho_{\operatorname{Tot}} \rho_{D}^{p-2}\right] \\
& =\frac{p-1}{2} \int \chi \rho_{D}^{p-2} \rho_{\operatorname{Tot}}\left(\tilde{\rho}^{(k)}\right)^{2}\left[\mu d+\mu \frac{\Lambda \chi}{\chi}+(p-2)\left(\frac{\partial_{\tau} \rho_{D}+\mu \Lambda \rho_{D}}{\rho_{D}}\right)\right. \\
& \left.\quad+\frac{\partial_{\tau} \rho_{\mathrm{Tot}}+\mu \Lambda \rho_{\mathrm{Tot}}}{\rho_{\mathrm{Tot}}}+O\left(\frac{1}{\langle Z\rangle^{r-1}}\right)\right] .
\end{aligned}
$$

We now claim the fundamental behavior

$$
\frac{\partial_{\tau} \rho_{D}+\mu \Lambda \rho_{D}}{\rho_{D}}=-\frac{2 \mu(r-1)}{p-1}+O\left(\frac{1}{\langle Z\rangle^{r}}\right)
$$

and

$$
\frac{\partial_{\tau} \rho_{\mathrm{Tot}}+\mu \Lambda \rho_{\mathrm{Tot}}}{\rho_{\mathrm{Tot}}}=-\frac{2 \mu(r-1)}{p-1}+O\left(\frac{1}{\langle Z\rangle^{r}}\right) .
$$


Assume (5.41), (5.42), we obtain

$$
\begin{aligned}
- & \int \chi H_{2} \Lambda \tilde{\rho}^{(k)}\left[(p-1) \rho_{D}^{p-2} \rho_{\mathrm{Tot}} \tilde{\rho}^{(k)}\right] \\
& +\frac{p-1}{2} \int \chi(p-2) \partial_{\tau} \rho_{D} \rho_{D}^{p-3} \rho_{\mathrm{Tot}}\left(\tilde{\rho}^{(k)}\right)^{2} \\
& +\frac{p-1}{2} \int \partial_{\tau} \rho_{\mathrm{Tot}} \tilde{\rho}^{p-2}\left(\tilde{\rho}^{(k)}\right)^{2} \\
= & \mu \frac{p-1}{2} \int \chi \rho_{D}^{p-2} \rho_{\mathrm{Tot}}\left(\tilde{\rho}^{(k)}\right)^{2}\left[d+\frac{\Lambda \chi}{\chi}-2(r-1)+O\left(\frac{1}{\langle Z\rangle^{r}}\right)\right] \\
= & \mu \frac{p-1}{2} \int \chi \rho_{D}^{p-2} \rho_{\operatorname{Tot}}\left(\tilde{\rho}^{(k)}\right)^{2}\left[d+\frac{\Lambda \chi}{\chi}-2(r-1)\right] \\
& +O\left(e^{-c_{k m} \tau}\right) .
\end{aligned}
$$

Proof of (5.41). From (4.9):

$$
\begin{aligned}
& \partial_{\tau} \rho_{D}+\mu \Lambda \rho_{D}=-\mu \Lambda \zeta\left(\frac{Z}{Z^{*}}\right) \rho_{P}(Z)+\mu \Lambda \zeta\left(\frac{Z}{Z^{*}}\right) \rho_{P}(Z), \\
& +\mu \zeta\left(\frac{Z}{Z^{*}}\right) \Lambda \rho_{P}=\mu \zeta\left(\frac{Z}{Z^{*}}\right) \Lambda \rho_{P}, \\
& \frac{\partial_{\tau} \rho_{D}+\mu \Lambda \rho_{D}}{\rho_{D}}=\mu \frac{\Lambda \rho_{P}}{\rho_{P}}=-\frac{2 \mu(r-1)}{p-1}+O\left(\frac{1}{\langle Z\rangle^{r}}\right)
\end{aligned}
$$

and (5.41) is proved.

Proof of (5.42). Recall (2.23)

$$
\partial_{\tau} \rho_{\mathrm{Tot}}=-\rho_{\mathrm{Tot}} \Delta \Psi_{\mathrm{Tot}}-\frac{\mu \ell(r-1)}{2} \rho_{\mathrm{Tot}}-\left(2 \partial_{Z} \Psi_{\mathrm{Tot}}+\mu Z\right) \partial_{Z} \rho_{\mathrm{Tot}}
$$

which yields

$$
\left|\frac{\partial_{\tau} \rho_{\mathrm{Tot}}+\mu \Lambda \rho_{\mathrm{Tot}}}{\rho_{\mathrm{Tot}}}+\frac{\mu \ell(r-1)}{2}\right|=\left|-\Delta \Psi_{\mathrm{Tot}}-2 \frac{\partial_{Z} \Psi_{\mathrm{Tot}} \partial_{Z} \rho_{\mathrm{Tot}}}{\rho_{\mathrm{Tot}}}\right|
$$

and (5.42) follows from (5.35).

$\Psi^{(k)}$ terms. Integrating by parts:

$$
\begin{aligned}
& \left|b^{2} \int \partial^{k} \Delta \rho_{D} \nabla \cdot\left(\chi \rho_{\mathrm{Tot}}^{2} \nabla \Psi^{(k)}\right)\right| \lesssim b^{2} \int \chi \rho_{T}^{2} \frac{\left|\nabla \Psi^{(k)}\right|}{\langle Z\rangle^{k+3}} \\
& \lesssim \int \chi \frac{\rho_{\mathrm{Tot}}^{2}\left|\nabla \Psi^{(k)}\right|^{2}}{\langle Z\rangle}+b^{4} \int \chi \frac{\rho_{\mathrm{Tot}}^{2}}{\langle Z\rangle^{2(k+3)-1}} \lesssim e^{-c_{k_{m}} \tau},
\end{aligned}
$$


where we used (5.28).

Next

$$
\mu(r-2) \int \Psi^{(k)} \nabla \cdot\left(\chi \rho_{\mathrm{Tot}}^{2} \nabla \Psi^{(k)}\right)=-\mu(r-2) \int \chi \rho_{\mathrm{Tot}}^{2}\left|\nabla \Psi^{(k)}\right|^{2} .
$$

Similarly, using $\partial_{Z} H_{2}=O\left(\frac{1}{\langle Z\rangle^{r}}\right)$ :

$$
\begin{aligned}
k & \int H_{2} \Psi^{(k)} \nabla \cdot\left(\chi \rho_{\mathrm{Tot}}^{2} \nabla \Psi^{(k)}\right) \\
= & -k\left[\int \chi \mu\left[1+O\left(\frac{1}{\langle Z\rangle^{\frac{1}{2}}}\right)\right] \rho_{\mathrm{Tot}}^{2}\left|\nabla \Psi^{(k)}\right|^{2}\right. \\
& \left.+O\left(\int \chi \rho_{\mathrm{Tot}}^{2} \frac{\left|\Psi^{(k)}\right|^{2}}{\langle Z\rangle^{2 r-\frac{1}{2}}}\right)\right] \\
= & -k \mu \int \chi \rho_{\mathrm{Tot}}^{2}\left|\nabla \Psi^{(k)}\right|^{2}+O\left(e^{-c_{k_{m}} \tau}\right),
\end{aligned}
$$

where we also used that $r>2, k \neq 0$ and $\int \chi_{k, k, \sigma(k)} \rho_{\mathrm{Tot}}^{2} \frac{\left|\Psi^{(k)}\right|^{2}}{\langle Z\rangle^{2 r-\frac{1}{2}}} \lesssim \int \chi_{k, k-1, \sigma(k)} \rho_{\mathrm{Tot}}^{2} \frac{\left|\nabla \Psi_{k-1}\right|^{2}}{\langle Z\rangle^{2 r-\frac{1}{2}-2}} \leq\|\tilde{\rho}, \Psi\|_{k, \sigma(k)+\frac{1}{2}}^{2}$

Then using (5.35):

$$
\begin{aligned}
& \left|\int 2 \chi \rho_{\mathrm{Tot}}^{2} \nabla \Psi \cdot \nabla \Psi^{(k)}\left(2 \frac{\nabla \rho_{\mathrm{Tot}}}{\rho_{\mathrm{Tot}}}+\frac{\nabla \chi}{\chi}\right) \cdot \nabla \Psi^{(k)}\right| \\
& \lesssim \int \chi \frac{\rho_{\mathrm{Tot}}^{2}\left|\nabla \Psi^{(k)}\right|^{2}}{\langle Z\rangle} \lesssim e^{-c_{k_{m}} \tau}
\end{aligned}
$$

and from (5.27), (5.35):

$$
\begin{aligned}
\left|\int 2 \chi \rho_{\text {Tot }} \nabla \Psi \cdot \nabla \Psi^{(k)}\left(\rho_{\text {Tot }} \Delta \Psi^{(k)}\right)\right| & \lesssim \int \chi\left|\nabla \Psi^{(k)}\right|^{2}\left(\left|\partial\left(\rho_{\text {Tot }}^{2} \nabla \Psi\right)\right|+\frac{\left|\rho_{\text {Tot }}^{2} \nabla \Psi\right|}{\langle Z\rangle}\right) \\
& \lesssim \int \chi \frac{\rho_{\text {Tot }}^{2}\left|\nabla \Psi^{(k)}\right|^{2}}{\langle Z\rangle^{2}} \lesssim e^{-c_{k_{m}} \tau} .
\end{aligned}
$$


We now carefully compute from (5.27) again:

$$
\begin{aligned}
\int & \chi \rho_{\mathrm{Tot}} H_{2} \Lambda \Psi^{(k)}\left(2 \nabla \rho_{\mathrm{Tot}} \cdot \nabla \Psi^{(k)}+\rho_{\mathrm{Tot}} \Delta \Psi^{(k)}\right) \\
& +\int H_{2} \Lambda \Psi^{(k)} \rho_{\mathrm{Tot}}^{2} \nabla \chi \cdot \nabla \Psi^{(k)} \\
= & 2 \sum_{i, j} \int \chi \rho_{\mathrm{Tot}} H_{2} Z_{j} \partial_{j} \Psi^{(k)} \partial_{i} \rho_{\mathrm{Tot}} \partial_{i} \Psi^{(k)} \\
- & \sum_{i, j} \int \partial_{i}\left(\chi Z_{j} H_{2} \rho_{\mathrm{Tot}}^{2}\right) \partial_{i} \Psi^{(k)} \partial_{j} \Psi^{(k)} \\
& +\frac{1}{2} \int \nabla \cdot\left(\chi Z H_{2} \rho_{\mathrm{Tot}}^{2}\right)\left|\nabla \Psi^{(k)}\right|^{2}+\sum_{i, j} H_{2} \rho_{\mathrm{Tot}}^{2} Z_{j} \partial_{j} \Psi^{(k)} \partial_{i} \chi \partial_{i} \Psi^{(k)} \\
= & \sum_{i, j} H_{2} \partial_{j} \Psi^{(k)} \partial_{i} \Psi^{(k)}\left[2 \chi \rho_{\mathrm{Tot}} \partial_{i} \rho \mathrm{Tot} Z_{j}-\partial_{i} \chi Z_{j} \rho_{\mathrm{Tot}}^{2}\right. \\
& \left.-\chi \delta_{i j} \rho_{\mathrm{Tot}}^{2}-2 \chi Z_{j} \rho_{\mathrm{Tot}} \partial_{i} \rho_{\mathrm{Tot}}-\chi Z_{j} \frac{\partial_{i} H_{2}}{H_{2}} \rho_{\mathrm{Tot}}^{2}+Z_{j} \rho_{\mathrm{Tot}}^{2} \partial_{i} \chi\right] \\
& +\frac{1}{2} \int \chi H_{2} \rho_{\mathrm{Tot}}^{2}\left|\nabla \Psi^{(k)}\right|^{2}\left[d+\frac{\Lambda \chi}{\chi}+\frac{\Lambda H_{2}}{H_{2}}+2 \frac{\Lambda \rho_{\mathrm{Tot}}}{\rho_{\mathrm{Tot}}}\right] \\
= & \frac{1}{2} \mu \int \chi \rho_{\mathrm{Tot}}^{2}\left|\nabla \Psi^{(k)}\right|^{2}\left[d-2+\frac{\Lambda \chi}{\chi}+2 \frac{\Lambda \rho_{\mathrm{Tot}}}{\rho_{\mathrm{Tot}}}+O\left(\frac{1}{\langle Z\rangle^{r}}\right)\right]
\end{aligned}
$$

Hence the final formula recalling (5.42):

$$
\begin{aligned}
\int & \chi \rho_{\mathrm{Tot}} H_{2} \Lambda \Psi^{(k)}\left(2 \nabla \rho_{\mathrm{Tot}} \cdot \nabla \Psi^{(k)}+\rho_{\mathrm{Tot}} \Delta \Psi^{(k)}\right) \\
& +\int H_{2} \Lambda \Psi^{(k)} \rho_{\mathrm{Tot}}^{2} \nabla \chi \cdot \nabla \Psi^{(k)}+\int \chi \partial_{\tau} \rho_{\mathrm{Tot}} \rho_{\mathrm{Tot}}\left|\nabla \Psi^{(k)}\right|^{2} \\
= & \int \mu \chi \rho_{\mathrm{Tot}}^{2}\left|\nabla \Psi^{(k)}\right|^{2}\left[\frac{d-2}{2}+\frac{1}{2} \frac{\Lambda \chi}{\chi}+\frac{\Lambda \rho_{\mathrm{Tot}}}{\rho_{\mathrm{Tot}}}+\frac{1}{\mu} \frac{\partial_{\tau} \rho_{\mathrm{Tot}}}{\rho_{\mathrm{Tot}}}+O\left(\frac{1}{\langle Z\rangle^{r}}\right)\right] \\
= & \int \mu \chi \rho_{\mathrm{Tot}}^{2}\left|\nabla \Psi^{(k)}\right|^{2}\left[\frac{d-2}{2}+\frac{1}{2} \frac{\Lambda \chi}{\chi}-\frac{2(r-1)}{p-1}+O\left(\frac{1}{\langle Z\rangle^{r}}\right)\right] \\
= & \int \mu \chi \rho_{\mathrm{Tot}}^{2}\left|\nabla \Psi^{(k)}\right|^{2}\left[\frac{d-2}{2}+\frac{1}{2} \frac{\Lambda \chi}{\chi}-\frac{2(r-1)}{p-1}\right]+O\left(e^{-c_{k_{m}} \tau}\right) .
\end{aligned}
$$

Loss of derivatives terms. We integrate by parts the non linear term which must loose derivatives: 


$$
\begin{aligned}
& b^{2}\left|\int \rho_{\mathrm{Tot}} \nabla \chi \cdot \nabla \Psi^{(k)} \Delta \tilde{\rho}^{(k)}\right| \\
& \lesssim b^{2}\left|\int \rho_{\mathrm{Tot}} \Delta \Psi^{(k)} \nabla \chi \cdot \nabla \tilde{\rho}^{(k)}\right|+b^{2} \int \chi \frac{\rho_{\mathrm{Tot}}}{\langle Z\rangle^{2}}\left|\nabla \Psi^{(k)}\right|\left|\nabla \tilde{\rho}^{(k)}\right| \\
& \lesssim b^{3} \int \chi\left|\nabla \tilde{\rho}^{(k)}\right|^{2}+b\left[\int \chi \frac{\rho_{\mathrm{Tot}}^{2}\left|\Delta \Psi^{(k)}\right|^{2}}{\langle Z\rangle^{2}}+\int \chi \frac{\rho_{\mathrm{Tot}}^{2}\left|\nabla \Psi^{(k)}\right|^{2}}{\langle Z\rangle^{4}}\right] \\
& \lesssim e^{-c_{k_{m}} \tau}+b \int \chi \frac{\rho_{\mathrm{Tot}}^{2}\left|\Delta \Psi^{(k)}\right|^{2}}{\langle Z\rangle^{2}} .
\end{aligned}
$$

We now use (5.20) for $0 \leq k \leq k_{m}-1$ which implies

$$
\begin{aligned}
\int \chi_{k, k, \sigma(k)} \frac{\rho_{\operatorname{Tot}}^{2}\left|\Delta \Psi^{(k)}\right|^{2}}{\langle Z\rangle^{2}} & \leq \int \chi_{k+1, k+1, \sigma(k+1)} \rho_{\operatorname{Tot}}^{2}\left|\Delta \Psi^{(k)}\right|^{2} \\
& \lesssim\|\tilde{\rho}, \Psi\|_{k+1, \sigma(k+1)}^{2} \lesssim 1 .
\end{aligned}
$$

Hence

$$
\begin{aligned}
& b^{2}\left|\int \rho_{\mathrm{Tot}} \nabla \chi \cdot \nabla \Psi^{(k)} \Delta \tilde{\rho}^{(k)}\right|+b^{2}\left|\int \rho_{\mathrm{Tot}} \Delta \Psi^{(k)} \nabla \chi \cdot \nabla \tilde{\rho}^{(k)}\right| \\
& +b^{2}\left|\int \nabla \chi \cdot \nabla \tilde{\rho}^{(k)} \nabla \rho_{\mathrm{Tot}} \cdot \nabla \Psi^{(k)}\right| \lesssim e^{-c_{k_{m}} \tau} .
\end{aligned}
$$

Conclusion for linear terms. The energy identity (5.10) with the weight $\chi$ in (5.22), together with the estimates (5.29)-(5.34), (5.36)-(5.39), (5.43)-(5.48), (5.50) and (5.52) yields:

$$
\begin{aligned}
& \frac{1}{2} \frac{d}{d \tau}\left\{\int b^{2} \chi\left|\nabla \tilde{\rho}^{(k)}\right|^{2}+(p-1) \int \chi \rho_{D}^{p-2} \rho_{\mathrm{Tot}}\left(\tilde{\rho}^{(k)}\right)^{2}+\int \chi \rho_{\mathrm{Tot}}^{2}\left|\nabla \Psi^{(k)}\right|^{2}\right\} \\
& \leq e^{-c_{k_{m} \tau}}+\mu \int \chi\left[-k+\frac{d}{2}-(r-1)-\frac{2(r-1)}{p-1}+\frac{1}{2} \frac{\mu^{-1} \partial_{\tau} \chi+\Lambda \chi}{\chi}\right] \\
& \quad \times\left[b^{2}\left|\nabla \tilde{\rho}^{(k)}\right|^{2}+(p-1) \rho_{D}^{p-2} \rho_{\operatorname{Tot}}\left(\tilde{\rho}^{(k)}\right)^{2}+\rho_{\mathrm{Tot}}^{2}\left|\nabla \Psi^{(k)}\right|^{2}\right] \\
& \quad+\int F_{1} \chi(p-1) \rho_{D}^{p-2} \rho_{\mathrm{Tot}} \tilde{\rho}^{(k)}+b^{2} \int \chi \nabla F_{1} \cdot \nabla \tilde{\rho}^{(k)} \\
& \quad+\int \chi \rho_{T}^{2} \nabla F_{2} \cdot \nabla \Psi^{(k)} .
\end{aligned}
$$


Step $4 F_{1}$ terms. We recall (5.7) and claim the bound:

$$
\begin{aligned}
& (p-1) \int \chi F_{1}^{2} \rho_{D}^{p-2} \rho_{\mathrm{Tot}}+b^{2} \int \chi\left|\nabla F_{1}\right|^{2} \\
& \lesssim e^{-c_{k_{m}} \tau}\left[1+\|\tilde{\rho}, \Psi\|_{k, \sigma}^{2}\right]
\end{aligned}
$$

Source term induced by localization. Recall (5.2)

$$
\begin{aligned}
\tilde{\mathscr{E}}_{P, \rho} & =\partial_{\tau} \rho_{D}+\rho_{D}\left[\Delta \Psi_{P}+\mu \frac{\ell(r-1)}{2}+\left(2 \partial_{Z} \Psi_{P}+\mu Z\right) \frac{\partial_{Z} \rho_{D}}{\rho_{D}}\right] \\
& =\partial_{\tau} \rho_{D}+\mu \Lambda \rho_{D}+\mu \frac{\ell(r-1)}{2} \rho_{D}+\rho_{D} \Delta \Psi_{P}+2 \partial_{Z} \Psi_{P} \partial_{Z} \rho_{D} .
\end{aligned}
$$

From the proof of (5.41)

$$
\rho_{D}=\zeta\left(\frac{Z}{Z^{*}}\right) \rho_{P}, \quad \partial_{\tau} \rho_{D}+\mu \Lambda \rho_{D}=\mu \zeta\left(\frac{Z}{Z^{*}}\right) \Lambda \rho_{P} .
$$

Therefore, using the profile equation for $\rho_{P}$, we obtain

$$
\tilde{\varepsilon}_{P, \rho}=2 \frac{\Psi_{P}^{\prime}}{Z} \frac{Z}{Z^{*}} \zeta^{\prime} \rho_{P}
$$

From (2.10) and (2.19) we then conclude that

$$
\left|\partial^{k} \tilde{\mathscr{E}}_{P, \rho}\right| \lesssim \frac{\rho_{D}}{\langle Z\rangle^{k+r}} \mathbf{1}_{Z \geq Z^{*}}
$$

Hence, recalling (5.19) and (5.26):

$$
\begin{aligned}
\int & \chi \rho_{D}^{p-2} \rho_{\mathrm{Tot}}\left|\partial^{k} \tilde{\mathscr{E}}_{P, \rho}\right|^{2} \\
\lesssim & \int_{Z \geq Z^{*}} \frac{Z^{d-1} d Z}{Z^{-2 k+2\left(\frac{d}{2}-(r-1)+\tilde{v}-\frac{2(r-1)}{p-1}\right)}} \frac{1}{Z^{2 k+2 r}} \frac{(Z)^{-\frac{2(r-1)(p+1)}{p-1}}}{\left(\frac{Z}{Z^{*}}\right)^{(p-1) n_{P}-2(r-1)+2(r-2)-4 \tilde{v}}} \\
\lesssim & \int_{Z \geq Z^{*}} \frac{d Z}{Z^{2 r+2 \tilde{v}+1}\left(\frac{Z}{Z^{*}}\right)^{(p-1) n_{P}-2-4 \tilde{v}}} \lesssim\left(Z^{*}\right)^{-2 r-2 \tilde{v}} \lesssim e^{-c \tau} .
\end{aligned}
$$

Similarly, from (5.26):

$$
b^{2} \int \chi\left|\nabla \partial^{k} \tilde{\mathscr{E}}_{P, \rho}\right|^{2} \lesssim \int_{Z \geq Z^{*}} \frac{Z^{d-1} d Z}{Z^{2\left(\frac{d}{2}-1-\tilde{v}\right)+2+2 r}} \lesssim\left(Z^{*}\right)^{-2 r+2 \tilde{v}} \lesssim e^{-c \tau} .
$$


$\left[\partial^{k}, H_{1}\right]$ term. We use (5.5) to estimate:

$$
\mid \begin{aligned}
& \left|\left[\partial^{k}, H_{1}\right] \tilde{\rho}\right| \lesssim \sum_{j=0}^{k-1}\left|\partial^{j} \tilde{\rho} \partial^{k-j} H_{1}\right| \lesssim \sum_{j=0}^{k-1} \frac{\left|\partial^{j} \tilde{\rho}\right|}{\langle Z\rangle^{r+k-j}}, \\
& \left|\nabla\left[\partial^{k}, H_{1}\right] \tilde{\rho}\right| \lesssim \sum_{j=0}^{k} \frac{\left|\partial^{j} \tilde{\rho}\right|}{\langle Z\rangle^{1+r+k-j}} .
\end{aligned}
$$

Hence

$$
\begin{aligned}
(p-1) \int \chi \rho_{D}^{p-2} \rho_{\mathrm{Tot}}\left(\left[\partial^{k}, H_{1}\right] \tilde{\rho}\right)^{2} & \lesssim \sum_{j=0}^{k-1} \int \chi \rho_{D}^{p-1} \frac{\left|\partial^{j} \tilde{\rho}\right|^{2}}{\langle Z\rangle^{2(r+k-j)}} \\
& \lesssim\|\tilde{\rho}, \Psi\|_{k, \sigma(k)+r}^{2} \lesssim e^{-c_{k_{m}} \tau}
\end{aligned}
$$

and

$$
\begin{aligned}
& b^{2} \int \chi\left|\nabla\left(\left[\partial^{k}, H_{1}\right] \tilde{\rho}\right)\right|^{2} \lesssim b^{2} \sum_{j=0}^{k} \int \chi \frac{\left|\partial^{j} \tilde{\rho}\right|^{2}}{\langle Z\rangle^{2(1+r+k-j)}} \\
& \quad \lesssim b^{2} \int \chi \frac{\tilde{\rho}^{2}}{\langle Z\rangle^{2(1+r+k)}}+b^{2} \sum_{j=0}^{k} \int \chi \frac{\left|\partial^{j} \nabla \tilde{\rho}\right|^{2}}{\langle Z\rangle^{2(r+k-j)}} \\
& \quad \lesssim b^{2} \int \chi \frac{\rho_{D}^{2}}{\langle Z\rangle^{2(1+r+k)}}+e^{-c_{k_{m}} \tau} \lesssim e^{-c_{k_{m}} \tau}
\end{aligned}
$$

where we used the bootstrap bound (4.40), the decay of $b^{2}$ and (5.26). $\left[\partial^{k}, H_{2}\right]$ term. Similarly, from (5.5):

$$
\mid \begin{aligned}
& \left|\left[\partial^{k}, H_{2}\right] \Lambda \tilde{\rho}\right| \lesssim \sum_{j=0}^{k-1}\left|\partial^{j}(\Lambda \tilde{\rho}) \partial^{k-j} H_{2}\right| \lesssim \sum_{j=1}^{k} \frac{\left|\partial^{j} \tilde{\rho}\right|}{\langle Z\rangle^{r-1+k-j}} \\
& \left|\nabla\left[\partial^{k}, H_{2}\right] \Lambda \tilde{\rho}\right| \lesssim \sum_{j=1}^{k+1} \frac{\left|\partial^{j} \tilde{\rho}\right|}{\langle Z\rangle^{r+k-j}}
\end{aligned}
$$

Hence, using $r>1$ :

$$
\begin{aligned}
& (p-1) \int \chi \rho_{D}^{p-2} \rho_{\mathrm{Tot}}\left(\left[\partial^{k}, H_{2}\right] \Lambda \tilde{\rho}\right)^{2} \\
& \lesssim \sum_{j=1}^{k} \int \chi \rho_{D}^{p-1} \frac{\left|\partial^{j} \tilde{\rho}\right|^{2}}{\langle Z\rangle^{2(r-1+k-j)}} \lesssim e^{-c_{k_{m}} \tau},
\end{aligned}
$$

and 


$$
\begin{aligned}
& b^{2} \int \chi\left|\nabla\left(\left[\partial^{k}, H_{2}\right] \Lambda \tilde{\rho}\right)\right|^{2} \lesssim b^{2} \sum_{j=1}^{k+1} \int \chi \frac{\left|\partial^{j} \tilde{\rho}\right|^{2}}{\langle Z\rangle^{2(r+k-j)}} \\
& \quad=b^{2} \sum_{j=0}^{k} \int \chi \frac{\left|\partial^{j} \nabla \tilde{\rho}\right|^{2}}{\langle Z\rangle^{2(r-1+k-j)}} \lesssim\|\tilde{\rho}, \Psi\|_{k, \sigma+r-1}^{2} \lesssim e^{-c_{k_{m}} \tau}
\end{aligned}
$$

Nonlinear term. Changing indices, we need to estimate terms

$$
N_{j_{1}, j_{2}}=\partial^{j_{1}} \rho_{\mathrm{Tot}} \partial^{j_{2}} \nabla \Psi, \quad j_{1}+j_{2}=k+1, \quad 2 \leq j_{1}, j_{2} \leq k-1
$$

For the profile term:

$$
\left|\partial^{j_{1}} \rho_{D} \partial^{j_{2}} \nabla \Psi\right| \lesssim \rho_{D} \frac{\left|\partial^{j_{2}} \nabla \Psi\right|}{\langle Z\rangle^{j_{1}}}=\rho_{D} \frac{\left|\partial^{j_{2}} \nabla \Psi\right|}{\langle Z\rangle^{k+1-j_{2}}}
$$

and hence using from (5.19) the rough global bound:

$$
\rho_{D} \lesssim \frac{1}{\langle Z\rangle^{\frac{2(r-1)}{p-1}}}
$$

yields

$$
\begin{aligned}
\int(p-1) \chi N_{j_{1}, j_{2}}^{2} \rho_{D}^{p-2} \rho_{\mathrm{Tot}} & \lesssim \int \chi \frac{\rho_{\mathrm{Tot}}^{2}\left|\partial^{j_{2}} \nabla \Psi\right|^{2}}{\langle Z\rangle^{2\left(k+1-j_{2}\right)+2(r-1)}} \\
& =\int \chi \frac{\rho_{\mathrm{Tot}}^{2}\left|\partial^{j_{2}} \nabla \Psi\right|^{2}}{\langle Z\rangle^{2\left(k-j_{2}\right)+2 r}} \lesssim e^{-c_{k_{m}} \tau}
\end{aligned}
$$

Similarly, after taking a derivative:

$$
b^{2} \int \chi\left|\nabla N_{j_{1}, j_{2}}\right|^{2} \lesssim b^{2} \int \chi \frac{\rho_{\mathrm{Tot}}^{2}\left|\partial^{j_{2}} \nabla \Psi\right|^{2}}{\langle Z\rangle^{2\left(k+2-j_{2}\right)}} \lesssim e^{-c_{k_{m}} \tau}
$$

We now turn to the control of the nonlinear term. If $j_{1} \leq \frac{4 k_{m}}{9}$, then from (4.40):

$$
\int \chi \rho_{D}^{p-1}\left|\partial^{j_{1}} \tilde{\rho} \partial^{j_{2}} \nabla \Psi\right|^{2} \lesssim \int \chi \rho_{D}^{2} \frac{\left|\partial^{j_{2}} \nabla \Psi\right|^{2}}{\langle Z\rangle^{2\left(k+1-j_{2}\right)+2(r-1)}} \lesssim e^{-c_{k_{m}} \tau} .
$$

If $j_{2} \leq \frac{4 k_{m}}{9}$, then from (4.40) and $b=\frac{1}{\left(Z^{*}\right)^{r-2}}$ :

$$
\int \chi \rho_{D}^{p-1}\left|\partial^{j_{1}} \tilde{\rho} \partial^{j_{2}} \nabla \Psi\right|^{2} \lesssim \int_{Z \leq Z^{*}} \chi \rho_{D}^{p-1} \frac{\left|\partial^{j_{1}} \tilde{\rho}\right|^{2}}{\langle Z\rangle^{2\left(k+1+(r-2)-j_{1}\right)}}
$$




$$
+b^{2} \int_{Z \geq Z^{*}} \chi \rho_{D}^{p-1} \frac{\left|\partial^{j_{1}} \tilde{\rho}\right|^{2}}{\langle Z\rangle^{2\left(k+1-j_{1}\right)}} \lesssim e^{-c_{k_{m}} \tau}
$$

We may therefore assume $j_{1}, j_{2} \geq m_{0}=\frac{4 k_{m}}{9}+1$, which implies $k \geq m_{0}$ and $j_{1}, j_{2} \leq \frac{2 k_{m}}{3}$. From (4.29):

$$
\begin{aligned}
\sigma(k) & =-\alpha\left(k_{m}-k\right) \geq-\alpha\left(k_{m}-\frac{4 k_{m}}{9}\right)=-\frac{4}{5}\left(1-\frac{4}{9}\right) k_{m}+O_{k_{m} \rightarrow+\infty}(1) \\
& \geq-\frac{4 k_{m}}{9}+O_{k_{m} \rightarrow+\infty}(1) .
\end{aligned}
$$

From (4.41):

$$
\sigma(k)+n\left(j_{1}\right)+n\left(j_{2}\right) \geq-\frac{4 k_{m}}{9}+\frac{k_{m}}{4}+\frac{k_{m}}{4}+O_{k_{m} \rightarrow+\infty}(1) \geq \frac{k_{m}}{20}
$$

and hence from (4.40) and interpolating on $Z \leq Z_{c}^{*}$ with (4.39):

$$
\begin{aligned}
& \int \chi \rho_{D}^{p-1}\left|\partial^{j_{1}} \tilde{\rho} \partial^{j_{2}} \nabla \Psi\right|^{2} \lesssim e^{-c_{k_{m}} \tau}+\int_{Z \geq Z_{c}^{*}} \frac{Z^{d-1} d Z}{\langle Z\rangle^{\frac{k_{m}}{10}}} \\
& {\left[\mathbf{1}_{Z \leq Z^{*}}+\left(\frac{Z}{Z^{*}}\right)^{-(p-3) n_{P}-\frac{4(r-1)}{(p-1)}-2(r-2)-2(r-1)+4 \tilde{v}} \mathbf{1}_{Z \geq Z^{*}}\right] } \\
& \lesssim e^{-c_{k_{m}} \tau}
\end{aligned}
$$

The $b^{2}$ derivative term and the other nonlinear term in (5.7) are estimated similarly. We note that the relation

$$
k_{m} \gg n_{P} \gg 1
$$

ensures that the terms containing $k_{m}$ are dominant and eliminates the need to track the dependence on $n_{P}$. This concludes the proof of (5.54).

Step $5 F_{2}$ terms. We claim:

$$
\int \chi \rho_{\mathrm{Tot}}^{2}\left|\nabla F_{2}\right|^{2} \lesssim e^{-c_{k_{m}} \tau}\left[1+\|\tilde{\rho}, \Psi\|_{k+1, \sigma(k+1)}^{2}\right]
$$

Source term induced by localization. Recall (5.2):

$$
\tilde{\mathscr{E}}_{P, \Psi}=\left|\nabla \Psi_{P}\right|^{2}+\rho_{D}^{p-1}+e \Psi_{P}+\frac{1-e}{2} \Lambda \Psi_{P}-1=\rho_{D}^{p-1}-\rho_{P}^{p-1}
$$


which yields the rough bound

$$
\left|\nabla \partial^{k} \tilde{\mathscr{E}}_{P, \Psi}\right| \lesssim \frac{1}{\langle Z\rangle^{k+1+2(r-1)}} \mathbf{1}_{Z \geq Z^{*}}
$$

and hence, from (5.26),

$$
\begin{aligned}
\int \chi \rho_{\mathrm{Tot}}^{2}\left|\nabla \partial^{k} \tilde{\mathscr{E}}_{P, \Psi}\right|^{2} & \lesssim \int_{Z \geq Z^{*}} \frac{Z^{d-1}}{b^{2}\langle Z\rangle^{-2 k+2\left(\frac{d}{2}+\tilde{\nu}-1\right)}} \frac{\left(\frac{Z}{Z^{*}}\right)^{4 \tilde{\nu}}}{\langle Z\rangle^{2 k+2+4(r-1)}} \\
& \lesssim Z^{* 2(r-2)-4(r-1)+4 \tilde{v}} \lesssim e^{-c \tau}
\end{aligned}
$$

$\left[\partial^{k}, H_{2}\right] \Lambda \Psi$ term. From (5.5):

$$
\left|\nabla\left(\left[\partial^{k}, H_{2}\right] \Lambda \Psi\right)\right| \lesssim \sum_{j=1}^{k+1} \frac{\left|\partial^{j} \Psi\right|}{\langle Z\rangle^{r+k-j}} \lesssim \sum_{j=0}^{k} \frac{\left|\nabla \partial^{j} \Psi\right|}{\langle Z\rangle^{r+k-j-1}}
$$

and hence

$$
\int \chi \rho_{\mathrm{Tot}}^{2}\left|\nabla\left(\left[\partial^{k}, H_{2}\right] \Lambda \Psi\right)\right|^{2} \lesssim \sum_{j=0}^{k} \int \chi \rho_{\mathrm{Tot}}^{2} \frac{\left|\nabla \partial^{j} \Psi\right|^{2}}{\langle Z\rangle^{2(r-1+k-j)}} \lesssim e^{-c_{k_{m}} \tau}
$$

$\left[\partial^{k}, \rho_{D}^{p-2}\right] \tilde{\rho}-k(p-2) \rho_{D}^{p-3} \partial \rho_{D} \partial^{k-1} \tilde{\rho}$ term. By Leibnitz:

$$
\left|\left[\left[\partial^{k}, \rho_{D}^{p-2}\right] \tilde{\rho}-k(p-2) \rho_{D}^{p-3} \partial \rho_{D} \partial^{k-1} \tilde{\rho}\right]\right| \lesssim \sum_{j=0}^{k-2} \frac{\left|\partial^{j} \tilde{\rho}\right|}{\langle Z\rangle^{k-j}} \rho_{D}^{p-2}
$$

and, hence, taking a derivative:

$$
\begin{aligned}
\int & \chi \rho_{\text {Tot }}^{2}\left|\nabla\left[\left[\partial^{k}, \rho_{D}^{p-2}\right] \tilde{\rho}-k(p-2) \rho_{D}^{p-3} \partial \rho_{D} \partial^{k-1} \tilde{\rho}\right]\right|^{2} \\
& \lesssim \sum_{j=0}^{k-1} \int \chi \rho_{D}^{2(p-2)+2} \frac{\left|\partial^{j} \tilde{\rho}\right|^{2}}{\langle Z\rangle^{2(k-j)+2}} \\
& \lesssim \sum_{j=0}^{k-1} \int \chi \rho_{D}^{p-1} \frac{\left|\partial^{j} \tilde{\rho}\right|^{2}}{\langle Z\rangle^{2(k-j)+2}} \lesssim e^{-c_{k_{m}} \tau}
\end{aligned}
$$

Nonlinear $\Psi$ term. Let

$$
\partial N_{j_{1}, j_{2}}=\partial^{j_{1}} \nabla \Psi \partial^{j_{2}} \nabla \Psi, \quad j_{1}+j_{2}=k+1, \quad j_{1}, j_{2} \geq 1 .
$$


If $j_{1} \leq \frac{4 k_{m}}{9}$, then from (4.40):

$$
\int \chi \rho_{\mathrm{Tot}}^{2}\left|\nabla N_{j_{1}, j_{2}}\right|^{2} \lesssim \int \rho_{\mathrm{Tot}}^{2} \chi \frac{\left|\partial^{j_{2}} \nabla \Psi\right|^{2}}{\langle Z\rangle^{2\left(k+1-j_{2}\right)}} \lesssim\|\tilde{\rho}, \Psi\|_{k, \sigma+\frac{1}{2}}^{2} \lesssim e^{-c_{k_{m}} \tau} .
$$

The expression being symmetric in $j_{1}, j_{2}$, we may assume $j_{1}, j_{2} \geq m_{0}=$ $\frac{4 k_{m}}{9}+1, j_{1}, j_{2} \leq \frac{2 k_{m}}{3}$ and $k \geq m_{0}=\frac{4 k_{m}}{9}+1$. Using (4.40), (5.62) and arguing as above $\left(k_{m} \gg n_{P}\right)$ :

$$
\begin{aligned}
\int \chi \rho_{\mathrm{Tot}}^{2}\left|\nabla N_{j_{1}, j_{2}}\right|^{2} & \lesssim e^{-c \tau}+\int_{Z \geq Z_{c}^{*}} \frac{d Z}{\langle Z\rangle^{\frac{k_{m}}{10}}}\left[\mathbf{1}_{Z \leq Z^{*}}+\left(\frac{Z}{Z^{*}}\right)^{2 n_{P}+4 \tilde{v}} \mathbf{1}_{Z \geq Z^{*}}\right] \\
& \lesssim e^{-c_{k_{m}} \tau} .
\end{aligned}
$$

Quantum pressure term. We estimate from Leibniz:

$$
\begin{aligned}
& b^{2}\left|\partial^{k}\left(\frac{\Delta \rho_{\mathrm{Tot}}}{\rho_{\mathrm{Tot}}}\right)-\frac{\partial^{k} \Delta \rho_{\mathrm{Tot}}}{\rho_{\mathrm{Tot}}}+\frac{k \partial^{k-1} \Delta \rho_{\mathrm{Tot}} \partial \rho_{\mathrm{Tot}}}{\rho_{\mathrm{Tot}}^{2}}\right| \\
& \lesssim b^{2} \sum_{j_{1}+j_{2}=k, j_{2} \geq 2}\left|\partial^{j_{1}} \Delta \rho_{\mathrm{Tot}} \partial^{j_{2}}\left(\frac{1}{\rho_{\mathrm{Tot}}}\right)\right| .
\end{aligned}
$$

and using the Faa-di Bruno formula:

$$
\left|\partial^{j_{2}}\left(\frac{1}{\rho_{\mathrm{Tot}}}\right)\right| \lesssim \frac{1}{\rho_{\text {Tot }}^{j_{2}+1}} \sum_{q_{1}+2 q_{2}+\cdots+j_{2} q_{j_{2}}=j_{2}} \Pi_{i=1}^{j_{2}}\left|\left(\partial^{i} \rho_{\mathrm{Tot}}\right)^{q_{i}}\right| .
$$

We decompose $\rho_{\mathrm{Tot}}=\rho_{D}+\tilde{\rho}$ and control the $\rho_{D}$ term using the bound

$$
\left|\partial^{i} \rho_{D}\right| \lesssim \frac{\rho_{D}}{\langle Z\rangle^{i}}
$$

which yields

$$
\frac{1}{\rho_{\mathrm{Tot}}^{j_{2}+1}} \sum_{m_{1}+2 m_{2}+\cdots+j_{2} m_{j_{2}}=j_{2}} \Pi_{i=1}^{j_{2}}\left|\left(\partial^{i} \rho_{D}\right)^{m_{i}}\right| \lesssim \frac{1}{\rho_{\mathrm{Tot}}\langle Z\rangle^{j_{2}}}
$$

and hence the corresponding contribution to (5.63):

$$
b^{4} \int \chi \rho_{\text {Tot }}^{2}\left\{\sum_{j_{1}+j_{2}=k, j_{2} \geq 2} \frac{\left|\partial^{j_{1}+1} \Delta \rho_{\mathrm{Tot}}\right|^{2}}{\rho_{\mathrm{Tot}}^{2}\langle Z\rangle^{2 j_{2}}}+\frac{\left|\partial^{j_{1}} \Delta \rho_{\mathrm{Tot}}\right|^{2}}{\rho_{\mathrm{Tot}}^{2}\langle Z\rangle^{2 j_{2}+2}}\right\}
$$




$$
\begin{aligned}
& \lesssim b^{4} \sum_{j_{1}+j_{2}=k, j_{2} \geq 2}\left[\int \chi \frac{\rho_{D}^{2} d Z}{\langle Z\rangle^{2 j_{2}+2\left(j_{1}+3\right)}}+\int \chi \frac{\left|\partial^{j_{1}+3} \tilde{\rho}\right|^{2}}{\langle Z\rangle^{2 j_{2}}}\right] \\
& \lesssim b^{4} \int \chi \frac{\rho_{D}^{2} d Z}{\langle Z\rangle^{2 k+6}}+b^{4} \sum_{j_{1}=2}^{k} \int \chi \frac{\left|\nabla \partial^{j_{1}} \tilde{\rho}\right|^{2}}{\langle Z\rangle^{2\left(k-j_{1}\right)+2}} \lesssim e^{-c_{k_{m}} \tau}
\end{aligned}
$$

where we used (5.28) in the last step.

We now turn to the control of the nonlinear term and consider

$$
N_{j_{1}, j_{2}}=b^{2}\left(\partial^{j_{1}+1} \Delta \rho_{\mathrm{Tot}}\right) \frac{1}{\rho_{\text {Tot }}^{j_{2}+1}} \sum_{q_{1}+2 q_{2}+\cdots+j_{2} q_{j_{2}}=j_{2}} \Pi_{i=1}^{j_{2}}\left(\partial^{i} \hat{\rho}\right)^{q_{i}},
$$

where $\hat{\rho}$ is either $\rho_{D}$ or $\tilde{\rho}$. In both cases we will use the weaker estimates (4.40).

First assume that $q_{i}=0$ whenever $i \geq \frac{4 k_{m}}{9}+1$, then from (4.40):

$$
\begin{aligned}
\left|N_{j_{1}, j_{2}}\right| & \lesssim b^{2}\left|\partial^{j_{1}+1} \Delta \rho_{\mathrm{Tot}}\right| \frac{1}{\rho_{\mathrm{Tot}}^{j_{2}+1}} \sum_{q_{1}+2 q_{2}+\cdots+j_{2} q_{j_{2}}=j_{2}} \Pi_{i=1}^{j_{2}}\left|\left(\partial^{i} \hat{\rho}\right)^{q_{i}}\right| \\
& \lesssim b^{2} \frac{\left|\partial^{j_{1}+1} \Delta \rho_{\mathrm{Tot}}\right|}{\rho_{\operatorname{Tot}}\langle Z\rangle^{j_{2}}}
\end{aligned}
$$

and the conclusion follows verbatim as above. Otherwise, there are at most two value $\frac{4 k_{m}}{9} \leq i_{1} \leq i_{2} \leq j_{2}$ with $q_{i_{1}}, q_{i_{2}} \neq 0$ and $q_{i_{1}}+q_{i_{2}} \leq 2$. Hence from (4.40):

$$
\begin{aligned}
\frac{1}{\rho_{\mathrm{Tot}}^{j_{2}+1}} \Pi_{i=1}^{j_{2}}\left|\left(\partial^{i} \hat{\rho}\right)^{q_{i}}\right| & \lesssim \frac{1}{\rho_{\mathrm{Tot}}^{j_{2}+1}}\left|\partial^{i_{1}} \hat{\rho}\right|^{q_{i_{1}}}\left|\partial^{i_{2}} \hat{\rho}\right|^{q_{i_{2}}} \Pi_{1 \leq i \leq j_{2}, i \notin\left\{i_{1}, i_{2}\right\}}\left(\frac{\rho_{D}}{\langle Z\rangle^{i}}\right)^{q_{i}} \\
& \lesssim\left(\frac{\left|\partial^{i_{1}} \hat{\rho}\right|}{\rho_{D}}\right)^{q_{i_{1}}}\left(\frac{\left|\partial^{i_{2}} \hat{\rho}\right|}{\rho_{D}}\right)^{q_{i_{2}}} \frac{1}{\rho_{\mathrm{Tot}}\langle Z\rangle^{j_{2}-\left(q_{i_{1}} i_{1}+q_{i_{2}} i_{2}\right)}} .
\end{aligned}
$$

Assume first $i_{2} \geq \frac{2 k_{m}}{3}+1$, then $q_{i_{1}}=0, q_{i_{2}}=1$ and $j_{1}+3 \leq \frac{4 k_{m}}{9}$ from which:

$$
\begin{aligned}
\int \chi \rho_{\text {Tot }}^{2}\left|N_{j_{1}, j_{2}}\right|^{2} & \lesssim b^{4} \int \chi \rho_{\text {Tot }}^{2}\left|\partial^{j_{1}+1} \Delta \rho_{\text {Tot }}\right|^{2} \frac{\left|\partial^{i_{2}} \hat{\rho}\right|^{2}}{\rho_{T}^{2}} \frac{1}{\rho_{T}^{2}\langle Z\rangle^{2\left(j_{2}-i_{2}\right)}} \\
& \lesssim b^{4} \int \chi \frac{\left|\partial^{i_{2}} \hat{\rho}\right|^{2}}{\langle Z\rangle^{2\left(j_{2}-i_{2}\right)+2\left(j_{1}+3\right)}} \\
& \lesssim b^{4} \int \chi \frac{\left|\partial^{i_{2}} \hat{\rho}\right|^{2}}{\langle Z\rangle^{2\left(k-i_{2}\right)+6}} \lesssim e^{-c_{k_{m}} \tau}
\end{aligned}
$$


There remains the case $\frac{4 k_{m}}{9}+1 \leq i_{1} \leq i_{2} \leq \frac{2 k_{m}}{3}$ which imply $j_{1}+3 \leq \frac{2 k_{m}}{3}$, and we distinguish cases:

- case $\left(m_{i_{1}}, m_{i_{2}}\right)=(0,1):$ if $j_{1}+3 \leq \frac{4 k_{m}}{9}$, we estimate

$$
\begin{aligned}
& \int \chi \rho_{\text {Tot }}^{2}\left|N_{j_{1}, j_{2}}\right|^{2} \lesssim b^{4} \int \chi \rho_{D}^{2}\left|\partial^{j_{1}+1} \Delta \rho_{\mathrm{Tot}}\right|^{2} \frac{\left|\partial^{i_{2}} \hat{\rho}\right|^{2}}{\rho_{T}^{2}} \frac{1}{\rho_{T}^{2}\langle Z\rangle^{2\left(j_{2}-i_{2}\right)}} \\
& \lesssim b^{4} \int \chi \frac{\left|\partial^{i_{2}} \hat{\rho}\right|^{2}}{\langle Z\rangle^{2\left(j_{2}-i_{2}\right)+2\left(j_{1}+3\right)}} \lesssim b^{4} \int \chi \frac{\left|\partial^{i_{2}} \hat{\rho}\right|^{2}}{\langle Z\rangle^{2\left(k-i_{2}\right)+6}} \lesssim e^{-c_{k_{m}} \tau}
\end{aligned}
$$

Otherwise, $\frac{4 k_{m}}{9}+1 \leq j_{1}+3 \leq \frac{2 k_{m}}{3}$. Since $j_{2} \geq \frac{4 k_{m}}{9}+1$, then necessarily $j_{2} \leq \frac{2 k_{m}}{3}$. Hence $\frac{4 k_{m}}{9}+1 \leq j_{1}+3 \leq \frac{2 k_{m}}{3}, \frac{4 k_{m}}{9}+1 \leq j_{2} \leq \frac{2 k_{m}}{3}$ and we estimate from (4.40):

$$
\begin{aligned}
\int \chi \rho_{\text {Tot }}^{2}\left|N_{j_{1}, j_{2}}\right|^{2} \lesssim & b^{4} \int \frac{Z^{d-1} d Z}{\langle Z\rangle^{2\left(\sigma(k)+\frac{k_{m}}{4}+\frac{k_{m}}{4}+j_{2}-i_{2}\right)}} \\
& {\left[\mathbf{1}_{Z \leq Z^{*}}+\left(\frac{Z}{Z^{*}}\right)^{2 n_{P}+4 \tilde{v}} \mathbf{1}_{Z \geq Z^{*}}\right] \lesssim b^{4} \lesssim e^{-c_{k_{m}} \tau}, }
\end{aligned}
$$

where we once again used that in this range of $k$

$$
\sigma(k)+\frac{k_{m}}{2} \geq \frac{k_{m}}{20}, \quad k_{m} \gg n_{P} \gg 1
$$

- case $m_{i_{1}}+m_{i_{2}}=2$ : we use (4.40) and estimate crudely:

$$
\begin{aligned}
& \int \chi \rho_{\mathrm{Tot}}^{2}\left|N_{j_{1}, j_{2}}\right|^{2} \lesssim b^{4} \int \chi\left|\partial^{j_{1}+1} \Delta \rho_{\mathrm{Tot}}\right|^{2}\left(\frac{1}{\langle Z\rangle^{\frac{k_{m}}{4}}}\right)^{4} \\
& \lesssim b^{4} \int \frac{Z^{d-1} d Z}{\langle Z\rangle^{2\left(\sigma(k)+\frac{k_{m}}{2}\right)}} \\
& \times\left[\mathbf{1}_{Z \leq Z^{*}}+\left(\frac{Z}{Z^{*}}\right)^{2 n_{P}+4 \tilde{v}} \mathbf{1}_{Z \geq Z^{*}}\right] \lesssim b^{4} \lesssim e^{-c_{k_{m}} \tau} .
\end{aligned}
$$

$\mathrm{NL}(\tilde{\rho})$ term. We expand, using, according to our assumptions, that the power of the nonlinear term is an integer $p \geq 3$ :

$$
\mathrm{NL}(\tilde{\rho})=\left(\rho_{D}+\tilde{\rho}\right)^{p-1}-\rho_{D}^{p-1}-(p-1) \rho_{D}^{p-2} \tilde{\rho}=\sum_{q=2}^{p-1} c_{q} \tilde{\rho}^{q} \rho_{D}^{p-1-q}
$$


and hence by Leibniz:

$$
\begin{aligned}
\partial^{k} \mathrm{NL}(\tilde{\rho}) & =\sum_{q=2}^{p-1} \sum_{j_{1}+j_{2}=k} c_{q, j_{1}, j_{2}} \partial^{j_{1}}\left(\tilde{\rho}^{q}\right) \partial^{j_{2}}\left(\rho_{D}^{p-1-q}\right) \\
& =\sum_{q=2}^{p-1} \sum_{j_{1}+j_{2}=k} \sum_{\ell_{1}+\cdots+\ell_{q}=j_{1}} \partial^{\ell_{1}} \tilde{\rho} \cdots \partial^{\ell_{q}} \tilde{\rho} \partial^{j_{2}}\left(\rho_{D}^{p-1-q}\right) .
\end{aligned}
$$

Let

$$
N_{\ell_{1}, \ldots, \ell_{q}, j_{1}, q}=\partial^{\ell_{1}} \tilde{\rho} \cdots \partial^{\ell_{q}} \tilde{\rho} \partial^{j_{2}}\left(\rho_{D}^{p-1-q}\right), \quad \ell_{1} \leq \cdots \leq \ell_{q}
$$

then

$$
\left|\nabla N_{\ell_{1}, \ldots, \ell_{q}, j_{1}, q}\right| \lesssim\left|N_{\ell_{1}, \ldots, \ell_{q}, j_{1}, q}^{(1)}\right|+\left|N_{\ell_{1}, \ldots, \ell_{q}, j_{1}, q}^{(2)}\right|
$$

with

$\left|N_{\ell_{1}, \ldots, \ell_{q}, j_{1}, q}^{(1)}\right| \lesssim\left|\partial^{m_{1}} \tilde{\rho} \cdots \partial^{m_{q}} \tilde{\rho}\right| \frac{\rho_{D}^{p-1-q}}{\langle Z\rangle^{j_{2}}}, \quad \mid \begin{aligned} & 0 \leq m_{1} \leq \cdots \leq m_{q} \leq k+1 \\ & m_{1}+\cdots+m_{q}=j_{1}+1 .\end{aligned}$

We estimate $N_{\ell_{1}, \ldots, \ell_{q}, j_{1}, q}^{(1)}$, the other term being estimated similarly. We distinguish cases.

- case $m_{q} \leq \frac{4 k_{m}}{9}$, then from (4.40):

$$
\left|N_{m_{1}, \ldots, m_{q}, j_{1}, q}^{(1)}\right| \lesssim \frac{\tilde{\rho}^{q}}{\langle Z\rangle^{j_{1}+1}} \frac{\rho_{D}^{p-1-q}}{\langle Z\rangle^{j_{2}}} \lesssim \frac{\rho_{D}^{p-1}}{\langle Z\rangle^{k+1}}
$$

and hence, from (5.19) and (5.25), the contribution of this term is given by

$$
\begin{aligned}
& \int \chi \rho_{\text {Tot }}^{2}\left|N_{m_{1}, \ldots, m_{q}, j_{1}, q}^{(1)}\right|^{2} \lesssim e^{-c \tau} \\
& \quad+\int_{Z \geq Z_{c}^{*}} \frac{Z^{d-1} d Z}{\langle Z\rangle^{2 \sigma(k)+2(k+1)}} \frac{\mathbf{1}_{Z \leq Z^{*}+\left(\frac{Z}{Z^{*}}\right)^{-2(p-1) n_{P}-4(r-1)-2(r-2)+4 \tilde{v}}} \mathbf{1}_{Z \geq Z^{*}}}{\langle Z\rangle^{4(r-1)+\frac{4(r-1)}{p-1}}} \\
& \lesssim e^{-c \tau}+\int_{Z \geq Z_{c}^{*}} \frac{d Z}{\langle Z\rangle^{2(r-1)+3}} \lesssim e^{-c_{k_{m}} \tau} .
\end{aligned}
$$

We now assume $m_{q} \geq \frac{4 k_{m}}{9}+1$ and recall $m_{q} \leq j_{1}+1 \leq k+1 \leq k_{m}$. 
- case $m_{q-1} \leq \frac{4 k_{m}}{9}$, then from (4.40):

$\left|N_{m_{1}, \ldots, m_{q}, j_{1}, q}^{(1)}\right| \lesssim \frac{\rho_{D}^{q-1}}{\langle Z\rangle^{j_{1}-m_{q}+1}}\left|\partial^{m_{q}} \tilde{\rho}\right| \frac{\rho_{D}^{p-1-q}}{\langle Z\rangle^{j_{2}}} \lesssim\left|\partial^{m_{q}} \tilde{\rho}\right| \rho_{D}^{p-2} \frac{1}{\langle Z\rangle^{k+1-m_{q}}}$.

If $m_{q} \leq k$ then

$$
\int \chi \rho_{\text {Tot }}^{2}\left|N_{m_{1}, \ldots, m_{q}, j_{1}, q}^{(1)}\right|^{2} \lesssim \int \chi \rho_{D}^{2} \frac{\left|\partial^{m_{q}} \tilde{\rho}\right|^{2}}{\langle Z\rangle^{2\left(k-m_{q}+1\right)+\frac{4(r-1)(p-2)}{p-1}}} \lesssim e^{-c_{k_{m}} \tau}
$$

On the other hand, if $m_{q}=k+1$, then, using (5.20)

$$
\begin{aligned}
& \int \chi_{k, k, \sigma(k)} \rho_{\mathrm{Tot}}^{2}\left|N_{m_{1}, \ldots, m_{q}, j_{1}, q}^{(1)}\right|^{2} \lesssim \int \chi_{k, k, \sigma(k)} \rho_{D}^{2} \rho_{D}^{2(p-3)} \tilde{\rho}^{2}\left|\partial^{k+1} \tilde{\rho}\right|^{2} \\
& \lesssim \int_{Z<Z_{c}^{*}} \chi_{k+1, k+1, \sigma(k+1)} \rho_{D}^{2(p-2)}\langle Z\rangle^{2} \tilde{\rho}^{2}\left|\partial^{k+1} \tilde{\rho}\right|^{2} \\
& \quad+\int_{Z>Z_{c}^{*}} \chi_{k+1, k+1, \sigma(k+1)} \rho_{D}^{p-1} \frac{\left|\partial^{k+1} \tilde{\rho}\right|^{2}}{\langle Z\rangle^{-2+2(r-1)}} \\
& \lesssim e^{-c_{k_{m}} \tau}\|\tilde{\rho}, \Psi\|_{k+1, \sigma(k+1)}^{2},
\end{aligned}
$$

where we used the following interpolation bound

$$
\|\tilde{\rho}\|_{L^{\infty}\left(Z \leq Z_{c}^{*}\right)} \lesssim e^{-c \tau}
$$

the estimate

$$
\tilde{\rho} \lesssim \rho_{D} \lesssim\langle Z\rangle^{-\frac{2(r-1)}{p-1}}
$$

and the condition

$$
-2+2(r-1)>0
$$

which follows from $r>2$.

- case $m_{q-1} \geq \frac{4 k_{m}}{9}+1$, then necessarily $m_{q-2} \leq \frac{4 k_{m}}{9}<\frac{4 k_{m}}{9}+1 \leq m_{q-1} \leq$ $m_{q}<\frac{2 k_{m}}{3}$ and $k \geq \frac{4 k_{m}}{9}+1$. Hence

$$
\left|N_{m_{1}, \ldots, m_{q}, j_{1}, q}^{(1)}\right| \lesssim \frac{\rho_{D}^{q}}{\langle Z\rangle^{\frac{k_{m}}{4}+\frac{k_{m}}{4}}} \rho_{D}^{p-1-q} \lesssim \frac{\rho_{D}^{p-1}}{\langle Z\rangle^{\frac{k_{m}}{2}}} .
$$


The integral for $Z<Z_{c}^{*}$ is estimated as above, and we further estimate from (5.25) and (5.62), using that $k_{m} \gg n_{P} \gg 1$,

$$
\int_{Z \geq Z_{c}^{*}} \chi \rho_{D}^{2}\left|N_{m_{1}, \ldots, m_{q}, j_{1}, q}^{(1)}\right|^{2} \lesssim \int_{Z \geq Z_{c}^{*}} \frac{d Z}{\langle Z\rangle^{\frac{k_{m}}{20}}} \lesssim e^{-c_{k_{m}} \tau} .
$$

This concludes the proof of (5.63).

Step 6 Conclusion. Injecting (5.54) and (5.63) into (5.53) yields:

$$
\begin{aligned}
\frac{1}{2} & \frac{d}{d \tau}\left\{\int b^{2} \chi\left|\nabla \tilde{\rho}^{(k)}\right|^{2}+(p-1) \int \chi \rho_{D}^{p-2} \rho_{\operatorname{Tot}}\left(\tilde{\rho}^{(k)}\right)^{2}+\int \chi \rho_{\mathrm{Tot}}^{2}\left|\nabla \Psi^{(k)}\right|^{2}\right\} \\
\leq & \mu \int \chi\left[-k+\frac{d}{2}-(r-1)-\frac{2(r-1)}{p-1}+\frac{1}{2} \frac{\mu^{-1} \partial_{\tau} \chi+\Lambda \chi}{\chi}\right] \\
& \times\left[b^{2}\left|\nabla \tilde{\rho}^{(k)}\right|^{2}+(p-1) \rho_{D}^{p-2} \rho_{\operatorname{Tot}}\left(\tilde{\rho}^{(k)}\right)^{2}+\rho_{\mathrm{Tot}}^{2}\left|\nabla \Psi^{(k)}\right|^{2}\right]+e^{-c_{k_{m} \tau}} .
\end{aligned}
$$

We now compute, noting that $\partial_{\tau} Z^{*}=\mu Z^{*}$ and that $\xi_{k}$ only depends on $\tau$ through $Z^{*}$ :

$$
\begin{aligned}
& \partial_{\tau} \chi+\mu \Lambda \chi \\
& =\frac{1}{\langle Z\rangle^{2 \sigma(k)}}\left[\partial_{\tau} \xi_{k}\left(\frac{Z}{Z^{*}}\right)+\mu \Lambda \xi_{k}\left(\frac{Z}{Z^{*}}\right)\right]+\xi_{k}\left(\frac{Z}{Z^{*}}\right) \mu \Lambda\left(\frac{1}{\langle Z\rangle^{2 \sigma(k)}}\right) \\
& =\xi_{k}\left(\frac{Z}{Z^{*}}\right) \mu \Lambda\left(\frac{1}{\langle Z\rangle^{2 \sigma(k)}}\right) .
\end{aligned}
$$

Hence recalling (5.23):

$$
\begin{aligned}
k & -\frac{d}{2}+r-1+\frac{2(r-1)}{p-1}-\frac{1}{2} \frac{\mu^{-1} \partial_{\tau} \chi+\Lambda \chi}{\chi} \\
& =k+\sigma(k)-\frac{d}{2}+r-1+\frac{2(r-1)}{p-1}+O\left(\frac{1}{\langle Z\rangle}\right) \\
& \geq \sigma_{v}-\frac{d}{2}+r-1+\frac{2(r-1)}{p-1}+O\left(\frac{1}{\langle Z\rangle}\right) \geq \tilde{v}+O\left(\frac{1}{\langle Z\rangle}\right) .
\end{aligned}
$$

Using that $k \leq k_{m}-1$ and the interpolation bound (5.17) we may absorb the $O\left(\frac{1}{\langle Z\rangle}\right)$ term and (5.13) is proved.

\section{Pointwise bounds}

We are now in position to close the control of the pointwise bounds (4.40). We start with inner bounds $|x| \lesssim 1$ : 
Lemma 6.1 (Interior pointwise bounds) For all $0 \leq k \leq \frac{2 k_{m}}{3}$ :

$$
\mid \begin{aligned}
& \forall 0 \leq k \leq \frac{2 k_{m}}{3}, \quad\left\|\frac{\langle Z\rangle^{n(k)} \partial_{Z}^{k} \rho}{\rho_{P}}\right\|_{L^{\infty}\left(Z \leq Z^{*}\right)} \leq d_{0} \\
& \forall 1 \leq k \leq \frac{2 k_{m}}{3}, \quad\left\|\langle Z\rangle^{n(k)}\langle Z\rangle^{r-2} \partial_{Z}^{k} \Psi\right\|_{L^{\infty}\left(Z \leq Z^{*}\right)} \leq d_{0}
\end{aligned}
$$

where $d_{0}$ is a smallness constant depending on data.

Proof We integrate (5.13) in time and obtain, by choosing $0<\tilde{v} \ll c+c_{k_{m}}$, $\forall 0 \leq m \leq k_{m}-1$ :

$$
\begin{aligned}
I_{m}(\tau) & \leq e^{-2 \mu \tilde{\nu}\left(\tau-\tau_{0}\right)} I_{m}(0)+\frac{1}{c_{k_{m}}-2 \mu \tilde{\nu}}\left(e^{-2 \mu \tilde{\nu} \tau-c_{k_{m}} \tau_{0}}-e^{-c_{k_{m}} \tau}\right) \\
& \leq e^{-2 \mu \tilde{\nu}\left(\tau-\tau_{0}\right)} e^{-c \tau_{0}}+\frac{e^{-c_{k_{m}} \tau_{0}}}{c_{k_{m}}-2 \mu \tilde{\nu}} e^{-2 \mu \tilde{\nu} \tau} \leq d_{0} e^{-2 \mu \tilde{\nu} \tau}
\end{aligned}
$$

for some small constant $d_{0}$, which can be chosen to be arbitrarily small by increasing $\tau_{0}$. Below, we will adjust $\alpha_{0}$ to remain small while absorbing any other universal constant.

Recalling (5.16):

$$
\forall 0 \leq m \leq k_{m}-1, \quad\|\tilde{\rho}, \Psi\|_{m, \sigma(m)} \leq d_{0} e^{-\mu \tilde{\nu} \tau} .
$$

This, in particular, already implies bounds on the Sobolev and pointwise norms of $(\tilde{\rho}, \Psi)$ on compact sets: for any $Z_{K}<\infty$ and any $k \leq k_{m}-d$

$$
\|(\tilde{\rho}, \Psi)\|_{H^{k}\left(Z \leq Z_{K}\right)} \leq d_{0} e^{-\mu \tilde{\nu} \tau}, \quad\left\|\left(\partial^{k} \tilde{\rho}, \partial^{k} \Psi\right)\right\|_{L^{\infty}\left(Z \leq Z_{K}\right)} \leq d_{0} e^{-\mu \tilde{\nu} \tau}(6.4)
$$

case $m \leq \frac{4 k_{m}}{9}+1=m_{0}$. Recall (4.29), then (6.2) implies: $\forall 0 \leq m \leq m_{0}$,

$$
\begin{aligned}
& \left\|\langle Z\rangle^{m-\frac{d}{2}+\frac{2(r-1)}{p-1}-\tilde{v}} \partial_{Z}^{m} \rho\right\|_{L^{2}\left(Z \leq Z^{*}\right)}^{2}+\left\|\langle Z\rangle^{m-\frac{d}{2}+(r-1)-\tilde{v}} \partial_{Z}^{m+1} \Psi\right\|_{L^{2}\left(Z \leq Z^{*}\right)}^{2} \\
& \leq Q_{0} e^{-2 \mu \tilde{v} \tau} .
\end{aligned}
$$

We now write for any spherically symmetric function $u$ and $\gamma>\frac{d}{2}-1$ :

$$
\begin{aligned}
|u(Z)| \lesssim & |u(1)|+\int_{1}^{Z}\left|\partial_{Z} u\right| d \sigma \lesssim|u(1)| \\
& +\left(\int_{1 \leq \sigma \leq Z} \frac{\left|\partial_{Z} u\right|^{2}}{\tau^{2 \gamma}} \tau^{d-1} d \tau\right)^{\frac{1}{2}}\left(\int_{1 \leq \sigma \leq Z} \frac{\tau^{2 \gamma}}{\tau^{d-1}} d \tau\right)^{\frac{1}{2}} \\
\lesssim & |u(1)|+\langle Z\rangle^{\gamma+1-\frac{d}{2}}\left\|\frac{\partial_{Z} u}{\langle Z\rangle \gamma}\right\|_{L^{2}(1 \leq \sigma \leq Z)} .
\end{aligned}
$$


We pick $1 \leq m \leq m_{0}$ and apply this to $u=Z^{\frac{2(r-1)}{p-1}} Z^{m-1} \partial_{Z}^{m-1} \rho, \gamma+1=\frac{d}{2}+\tilde{v}$ and obtain for $Z \leq Z^{*}$ from (6.4) and (6.5):

$$
\begin{aligned}
\left|Z^{m-1+\frac{2(r-1)}{p-1}} \partial_{Z}^{m-1} \rho\right| & \lesssim e^{-c \tau}+\langle Z\rangle^{\tilde{\nu}}\left\|\frac{\partial_{Z}\left(Z^{\frac{2(r-1)}{p-1}+m-1} \partial_{Z}^{m-1} \rho\right)}{\langle Z\rangle^{\frac{d}{2}+\tilde{v}-1}}\right\|_{L^{2}\left(2 Z_{2} \leq Z \leq Z^{*}\right)} \\
& \lesssim e^{-c \tau}+\langle Z\rangle^{\tilde{\nu}}\left[\left\|\frac{\langle Z\rangle^{m+\frac{2(r-1)}{p-1}} \partial_{Z}^{m} \rho}{\langle Z\rangle^{\frac{d}{2}+\tilde{v}}}\right\|_{L^{2}\left(Z \leq Z^{*}\right)}\right. \\
& \left.+\left\|\frac{\langle Z\rangle^{m-1+\frac{2(r-1)}{p-1}} \partial_{Z}^{m-1} \rho}{\langle Z\rangle^{\frac{d}{2}+\tilde{v}}}\right\|_{L^{2}\left(Z \leq Z^{*}\right)}\right] \\
& \lesssim e^{-c \tau}+d_{0}\langle Z\rangle^{\tilde{v}} e^{-\mu \tilde{\nu} \tau} \leq e^{-c \tau}+d_{0}\left(\frac{\langle Z\rangle}{Z^{*}}\right)^{\tilde{v}} \lesssim d_{0}
\end{aligned}
$$

and hence

$$
\forall 0 \leq m \leq \frac{4 k_{m}}{9}, \quad\left\|\frac{Z^{m} \partial_{Z}^{m} \rho}{\rho_{P}}\right\|_{L^{\infty}\left(Z \leq Z^{*}\right)} \leq d_{0} .
$$

We similarly pick $1 \leq m \leq m_{0}$, apply (6.6) to $u=\langle Z\rangle^{r-2+m} \partial_{Z}^{m} \Psi, \gamma+1=$ $\frac{d}{2}+\tilde{v}$, and obtain for $Z \leq Z^{*}$ from (6.5):

$$
\begin{aligned}
\left|\langle Z\rangle^{r-2+m} \partial_{Z}^{m} \Psi\right| \lesssim & e^{-c \tau}+\langle Z\rangle^{\tilde{\nu}}\left\|\frac{\partial_{Z}\left(\langle Z\rangle^{r-2+m} \partial_{Z}^{m} \Psi\right)}{\langle Z\rangle^{\frac{d}{2}+\tilde{v}-1}}\right\|_{L^{2}} \\
& +\langle Z\rangle^{\tilde{v}}\left[\left\|\frac{\left.\langle Z\rangle^{r-3+m} \partial_{Z}^{m} \Psi\right)}{\langle Z\rangle^{\frac{d}{2}+\tilde{\nu}-1}}\right\|_{L^{2}\left(2 Z_{2} \leq Z \leq Z^{*}\right)}\right. \\
& \left.+\left\|\frac{\left.\langle Z\rangle^{r-2+m} \partial_{Z}^{m+1} \Psi\right)}{\langle Z\rangle^{\frac{d}{2}+\tilde{v}-1}}\right\|_{L^{2}\left(2 Z_{2} \leq Z \leq Z^{*}\right)}\right] \\
\lesssim & e^{-c \tau}+d_{0}\langle Z\rangle^{\tilde{v}} e^{-\mu \tilde{v} \tau} \leq e^{-c \tau}+d_{0}\left(\frac{\langle Z\rangle}{Z^{*}}\right)^{\tilde{v}} \leq d_{0}
\end{aligned}
$$

and hence

$$
\forall 1 \leq m \leq m_{0}=\frac{4 k_{m}}{9}+1, \quad\left\|\langle Z\rangle^{r-2+m} \partial_{Z}^{m} \Psi\right\|_{L^{\infty}\left(Z \leq Z^{*}\right)} \leq d_{0} .
$$


case $m_{0} \leq m \leq \frac{2 k_{m}}{3}+1$. Recall (5.23):

$$
\sigma(m)+m=-\alpha\left(k_{m}-m\right)+m=(\alpha+1)\left(m-m_{0}\right)+\sigma_{v}
$$

and rewrite the norm:

$$
\begin{aligned}
\|\tilde{\rho}, \Psi\|_{m, \sigma}^{2} \geq & \sum_{k=0}^{m} \int_{Z \leq Z^{*}} \frac{1}{\langle Z\rangle^{2(m-k+\sigma(m))}} \\
& \times\left[b^{2}\left|\nabla \tilde{\rho}^{(k)}\right|^{2}+(p-1) \rho_{D}^{p-2} \rho_{\mathrm{Tot}}\left(\tilde{\rho}^{(k)}\right)^{2}+\rho_{\mathrm{Tot}}^{2}\left|\nabla \Psi^{(k)}\right|^{2}\right] \\
= & \sum_{k=0}^{m} \int_{Z \leq Z^{*}} \frac{\langle Z\rangle^{2 k}}{\langle Z\rangle^{2(\alpha+1)\left(m-m_{0}\right)+2 \sigma_{\nu}}} \\
& \times\left[b^{2}\left|\nabla \tilde{\rho}^{(k)}\right|^{2}+(p-1) \rho_{D}^{p-2} \rho_{\operatorname{Tot}}\left(\tilde{\rho}^{(k)}\right)^{2}+\rho_{\mathrm{Tot}}^{2}\left|\nabla \Psi^{(k)}\right|^{2}\right] .
\end{aligned}
$$

We infer, using also (6.2):

$$
\begin{aligned}
& \int_{2 Z_{2} \leq Z \leq Z^{*}}\left|\frac{Z^{m-(\alpha+1)\left(m-m_{0}\right)} \partial_{Z}^{m} \rho}{\langle Z\rangle^{\frac{d}{2}-\frac{2(r-1)}{p-1}+\tilde{v}}}\right|^{2} \\
& \quad+\int_{Z \leq Z_{*}}\left|\frac{Z^{m-(\alpha+1)\left(m-m_{0}\right)}\langle Z\rangle^{r-1} \partial_{Z}^{m+1} \Psi}{\langle Z\rangle^{\frac{d}{2}+\tilde{v}}}\right|^{2} \\
& \lesssim\|\rho, \Psi\|_{m, \sigma(m)}^{2} \leq d_{0} e^{-2 \mu \tilde{\nu} \tau} .
\end{aligned}
$$

Observe that for $m_{0} \leq m \leq \frac{2 k_{m}}{3}+1$, from (4.30):

$$
\begin{aligned}
m & -(\alpha+1)\left(m-m_{0}\right)=m_{0}(1+\alpha)-\alpha m \geq m_{0}(1+\alpha)-\alpha\left(2 \frac{k_{m}}{3}+1\right) \\
& =k_{m}\left[\frac{4}{9}\left(1+\frac{4}{5}\right)-\frac{2}{3} \frac{4}{5}\right]+O_{k_{m} \rightarrow+\infty}(1) \\
& =\frac{4 k_{m}}{15}+O_{k_{m} \rightarrow+\infty}(1)>\frac{k_{m}}{4}+10 .
\end{aligned}
$$

We now apply (6.6), (6.7) to $m_{0}+1 \leq m \leq \frac{2 k_{m}}{3}+1$, $u=\langle Z\rangle^{m+\frac{2(r-1)}{p-1}-(\alpha+1)\left(m-m_{0}\right)-1} \partial_{Z}^{m-1} \rho, \gamma+1=\frac{d}{2}+\tilde{v}$ and obtain for $Z \leq Z^{*}$ :

$$
\left|\langle Z\rangle^{m+\frac{2(r-1)}{p-1}-(\alpha+1)\left(m-m_{0}\right)-1} \partial_{Z}^{m-1} \rho\right|
$$




$$
\begin{aligned}
& \lesssim e^{-c \tau}+\langle Z\rangle^{\tilde{v}}\left\|\frac{\partial_{Z}\left(\langle Z\rangle^{m+\frac{2(r-1)}{p-1}-(\alpha+1)\left(m-m_{0}\right)-1} \partial_{Z}^{m-1} \rho\right)}{\langle Z\rangle^{\frac{d}{2}+\tilde{v}-1}}\right\|_{L^{2}\left(Z \leq Z^{*}\right)} \\
& \lesssim e^{-c \tau}+\langle Z\rangle^{\tilde{v}}\left\|\frac{\langle Z\rangle^{m+\frac{2(r-1)}{p-1}-(\alpha+1)\left(m-m_{0}\right)} \partial_{Z}^{m} \rho}{\langle Z\rangle^{\frac{d}{2}+\tilde{v}}}\right\| \\
& +\langle Z\rangle^{\tilde{v}}\left\|\frac{\langle Z\rangle^{m+\frac{2(r-1)}{p-1}-(\alpha+1)\left(m-m_{0}\right)-1} \partial_{Z}^{m-1} \rho}{\langle Z\rangle^{\frac{d}{2}+\tilde{v}}}\right\| \\
& \lesssim d_{0}\left[1+\langle Z\rangle^{\tilde{v}} e^{-\mu \tilde{v} \tau}\right]
\end{aligned}
$$

and hence using (6.8) for $Z \leq Z^{*}$ :

$$
\left|\frac{Z^{\frac{k_{m}}{4}} \partial_{Z}^{m-1} \rho}{\rho_{D}}\right| \lesssim\left|Z^{m+\frac{2(r-1)}{p-1}-(\alpha+1)\left(m-m_{0}\right)-1} \partial_{Z}^{m-1} \rho\right| \lesssim \alpha_{0}\left[1+\left(\frac{Z}{Z^{*}}\right)^{\tilde{v}}\right] \lesssim \ell_{0}
$$

and hence

$$
\forall \frac{4 k_{m}}{9}+1 \leq m \leq \frac{2 k_{m}}{3},\left\|\frac{Z^{\frac{k_{m}}{4}} \partial_{Z}^{m} \rho}{\rho_{D}}\right\|_{L^{\infty}\left(Z \leq Z^{*}\right)} \leq d_{0}
$$

For the phase, we apply (6.6), (6.7) to $m_{0}+1 \leq m \leq \frac{2 k_{m}}{3}+1, \gamma+1=\frac{d}{2}+\tilde{v}$, $u=\langle Z\rangle^{r-1+m-(\alpha+1)\left(m-m_{0}\right)} \partial_{Z}^{m} \Psi$ and obtain:

$$
\begin{aligned}
& \langle Z\rangle^{r-1+m-(\alpha+1)\left(m-m_{0}\right)}\left|\partial_{Z}^{m} \Psi\right| \\
& \lesssim e^{-c \tau}+\langle Z\rangle^{\tilde{v}}\left\|\frac{\partial_{Z}\left(\langle Z\rangle^{r-1+m-(\alpha+1)\left(m-m_{0}\right)} \partial_{Z}^{m} \Psi\right)}{\langle Z\rangle^{\frac{d}{2}+\tilde{v}-1}}\right\|_{L^{2}\left(Z \leq Z^{*}\right)} \\
& \lesssim e^{-c \tau}+\langle Z\rangle^{\tilde{v}}\left\|\frac{\langle Z\rangle^{r-1+m-1-(\alpha+1)\left(m-m_{0}\right)} \partial_{Z}^{m} \Psi}{\langle Z\rangle^{\frac{d}{2}+\tilde{v}-1}}\right\|_{L^{2}\left(Z \leq Z^{*}\right)} \\
& +\langle Z\rangle^{\tilde{v}}\left\|\frac{\langle Z\rangle^{r-1+m-(\alpha+1)\left(m-m_{0}\right)} \partial_{Z}^{m+1} \Psi}{\langle Z\rangle^{\frac{d}{2}+\tilde{v}-1}}\right\| \|_{L^{2}\left(Z \leq Z^{*}\right)} \\
& \leq d_{0}\left[1+\langle Z\rangle^{\tilde{v}} e^{-\mu \tilde{v} \tau}\right]
\end{aligned}
$$

and hence for $m_{0}+1 \leq m \leq \frac{2 k_{m}}{3}$ from (6.8) for $Z \leq Z^{*}$ :

$$
\langle Z\rangle^{r-2+\frac{k m}{4}}\left|\partial_{Z}^{m} \Psi\right| \lesssim\langle Z\rangle^{r-1+m-(\alpha+1)\left(m-m_{0}\right)}\left|\partial_{Z}^{m} \Psi\right| \leq d_{0},
$$


which concludes the proof of (6.1).

Similar to the above, we also have the following exterior bounds for $|x| \geq 1$ :

Lemma 6.2 (Exterior pointwise bounds) There holds:

$$
\mid \begin{aligned}
& \forall 0 \leq k \leq \frac{2 k_{m}}{3}, \quad\left\|\frac{\langle Z\rangle^{n(k)} \partial_{Z}^{k} \rho}{\rho P}\right\|_{L^{\infty}\left(Z \geq Z^{*}\right)} \leq d_{0}, \\
& \forall 1 \leq k \leq \frac{2 k_{m}}{3}, \quad\left\|\frac{\langle Z\rangle^{n(k)} \partial_{Z}^{k} \Psi}{b}\right\|_{L^{\infty}\left(Z \geq Z^{*}\right)} \leq d_{0},
\end{aligned}
$$

where $d_{0}$ is a smallness constant depending on data.

Proof We start with the case $0 \leq k \leq \frac{4 k_{m}}{9}$. We have in that case

$$
\begin{aligned}
I_{k, \sigma(k)} \geq & \int_{Z \geq Z^{*}}\langle Z\rangle^{2 k-2 \sigma_{\nu}}\left(\frac{Z}{Z^{*}}\right)^{2 n_{P}-\frac{4(r-1)}{p-1}-2(r-2)+4 \tilde{\nu}} \\
& \times\left[b^{2}\left|\nabla \tilde{\rho}^{(k)}\right|^{2}+(p-1) \rho_{D}^{p-1}\left(\tilde{\rho}^{(k)}\right)^{2}+\rho_{D}^{2}\left|\nabla \Psi^{(k)}\right|^{2}\right]
\end{aligned}
$$

We observe from (4.9), (4.28), (5.12) and $b=Z^{* 2-r}$ that for $Z \geq Z^{*}$

$$
\begin{aligned}
& Z^{d-1}\langle Z\rangle^{2 k-2 \sigma_{v}}\left(\frac{Z}{Z^{*}}\right)^{2 n_{P}-\frac{4(r-1)}{p-1}-2(r-2)+4 \tilde{v}} b^{2}\left|\nabla \tilde{\rho}^{(k)}\right|^{2} \\
& \approx\langle Z\rangle^{d-1+2 k-2 \sigma_{v}-\frac{4(r-1)}{p-1}-2(r-2)+4 \tilde{v}} \frac{\left|\nabla \tilde{\rho}^{(k)}\right|^{2}}{\left(Z^{*}\right)^{4 \tilde{v}} \rho_{D}^{2}} \\
& =\langle Z\rangle^{1+2 \tilde{v}}\left(\frac{\langle Z\rangle^{k}\left|\nabla \tilde{\rho}^{(k)}\right|}{\left(Z^{*}\right)^{2 \tilde{v}} \rho_{D}}\right)^{2}
\end{aligned}
$$

Similarly,

$$
\begin{aligned}
& Z^{d-1}\langle Z\rangle^{2 k-2 \sigma_{v}}\left(\frac{Z}{Z^{*}}\right)^{2 n_{P}-\frac{4(r-1)}{p-1}-2(r-2)+4 \tilde{v}} \rho_{D}^{2}\left|\nabla \Psi^{(k)}\right|^{2} \\
& \approx\langle Z\rangle^{d-1+2 k-2 \sigma_{v}-\frac{4(r-1)}{p-1}-2(r-2)+4 \tilde{v}} \frac{\left|\nabla \Psi^{(k)}\right|^{2}}{\left(Z^{*}\right)^{4 \tilde{v}} b^{2}} \\
& =\langle Z\rangle^{1+2 \tilde{v}}\left(\frac{\langle Z\rangle^{k}\left|\nabla \Psi^{(k)}\right|}{\left(Z^{*}\right)^{2 \tilde{v}} b}\right)^{2} .
\end{aligned}
$$

Now, for a spherically symmetric function $u, Z \geq Z^{*}$ and an arbitrary $\lambda>0$

$$
|u(Z)|=\left|u\left(Z^{*}\right)+\int_{Z^{*}}^{Z} \partial Z u\right|
$$




$$
\begin{aligned}
& \leq\left|u\left(Z^{*}\right)\right|+\left(\int_{Z^{*}}^{Z} \tau^{1+2 \lambda}\left|\partial_{Z} u\right|^{2} d \tau\right)^{\frac{1}{2}}\left(\int_{Z^{*}}^{Z} \tau^{-1-2 \lambda} d \tau\right)^{\frac{1}{2}} \\
& \leq\left|u\left(Z^{*}\right)\right|+\left(Z^{*}\right)^{-\lambda}\left(\int_{Z^{*}}^{Z} \tau^{1+2 \lambda}\left|\partial_{Z} u\right|^{2} d \tau\right)^{\frac{1}{2}} .
\end{aligned}
$$

We apply this to $u=\left(\frac{\langle Z\rangle^{k} \tilde{\rho}^{(k)}}{\left(Z^{*}\right)^{2} \tilde{v} \rho_{D}}\right)$ for $k \geq 1$ and $\lambda=\tilde{v}$

$$
\begin{aligned}
& \left|\left(\frac{\langle Z\rangle^{k} \tilde{\rho}^{(k)}}{\left(Z^{*}\right)^{2 \tilde{v}} \rho_{D}}\right)(Z)\right| \leq\left|\left(\frac{\langle Z\rangle^{k} \tilde{\rho}^{(k)}}{\left(Z^{*}\right)^{2 \tilde{v}} \rho_{D}}\right)\left(Z^{*}\right)\right| \\
& \quad+\left(Z^{*}\right)^{-\tilde{v}}\left(\int_{Z^{*}}^{Z} \tau^{1+2 \tilde{v}}\left[\left(\frac{\langle\tau\rangle^{k}\left|\nabla \tilde{\rho}^{(k)}\right|}{\left(Z^{*}\right)^{2 \tilde{v}} \rho_{D}}\right)^{2}+\left(\frac{\langle\tau\rangle^{k-1} \tilde{\rho}^{(k)}}{\left(Z^{*}\right)^{2 \tilde{v}} \rho_{D}}\right)^{2}\right] d \tau\right)^{\frac{1}{2}} \\
& \quad \lesssim\left(Z^{*}\right)^{-2 \tilde{v}} d_{0}+\left(Z^{*}\right)^{-\tilde{v}}\left(I_{k, \sigma(k)}+I_{k-1, \sigma(k-1)}\right)^{\frac{1}{2}},
\end{aligned}
$$

where we used the already proved interior bounds (6.1). This, together with (6.2), immediately implies the exterior bound for $\partial_{Z}^{k} \rho$ and $1 \leq k \leq \frac{4 k_{m}}{9}+1$. The corresponding bound for $\partial_{Z}^{k} \Psi$ is obtained similarly using $u=\left(\frac{\langle Z\rangle^{k} \Psi^{(k)}}{\left(Z^{*}\right)^{2 \tilde{v}} b}\right)$ and $\lambda=\tilde{v}$. To prove the result for $\rho$ in the case of $k=0$ we note that the bootstrap assumptions imply that $\tilde{\rho} \rightarrow 0$, so that, together with the above estimate for $k=1$, we have, for $Z \geq Z_{*}$,

$$
|\tilde{\rho}(Z)|=\left|\int_{Z}^{+\infty} \partial_{Z} \tilde{\rho}\right| \leq \alpha_{0} \int_{Z}^{+\infty} \frac{\rho_{D}(\tau)}{\tau} d \tau \leq \alpha_{0} \rho_{D}(Z)
$$

as desired.

Finally, we consider the regime $\frac{4 k_{m}}{9}+1 \leq k \leq \frac{2 k_{m}}{3}$. We have in that case

$$
\begin{aligned}
I_{k, \sigma(k) \geq} & \int_{Z \geq Z^{*}}\langle Z\rangle^{2 \alpha\left(k_{m}-k\right)}\left(\frac{Z}{Z^{*}}\right)^{2 n_{P}-\frac{4(r-1)}{p-1}-2(r-2)+4 \tilde{v}} \\
& \times\left[b^{2}\left|\nabla \tilde{\rho}^{(k)}\right|^{2}+(p-1) \rho_{D}^{p-1}\left(\tilde{\rho}^{(k)}\right)^{2}+\rho_{D}^{2}\left|\nabla \Psi^{(k)}\right|^{2}\right]
\end{aligned}
$$

We observe, using $n(k)=k_{m} / 4$ in that range, for $Z \geq Z^{*}$

$$
Z^{d-1}\langle Z\rangle^{2 \alpha\left(k_{m}-k\right)}\left(\frac{Z}{Z^{*}}\right)^{2 n_{P}-\frac{4(r-1)}{p-1}-2(r-2)+4 \tilde{v}} b^{2}\left|\nabla \tilde{\rho}^{(k)}\right|^{2}
$$




$$
\approx\langle Z\rangle^{\varpi(k)}\left(\frac{\langle Z\rangle^{n(k)}\left|\nabla \tilde{\rho}^{(k)}\right|}{\left(Z^{*}\right)^{2 \tilde{v}} \rho_{D}}\right)^{2}
$$

and

$$
\begin{aligned}
& Z^{d-1}\langle Z\rangle^{2 \alpha\left(k_{m}-k\right)}\left(\frac{Z}{Z^{*}}\right)^{2 n_{P}-\frac{4(r-1)}{p-1}-2(r-2)+4 \tilde{v}} \rho_{D}^{2}\left|\nabla \Psi^{(k)}\right|^{2} \\
& \approx\langle Z\rangle^{\varpi(k)}\left(\frac{\langle Z\rangle^{k}\left|\nabla \Psi^{(k)}\right|}{\left(Z^{*}\right)^{2 \tilde{v}} b}\right)^{2}
\end{aligned}
$$

where

$$
\varpi(k):=2 \alpha\left(k_{m}-k\right)-\frac{k_{m}}{2}-2(r-2)+4 \tilde{v}-\frac{4(r-1)}{p-1}+d-1 .
$$

Since $k \leq \frac{2 k_{m}}{3}$, and in view of the control of $\alpha$ in (4.30), we have

$$
\varpi(k) \geq\left(\frac{8}{15}-\frac{1}{2}\right) k_{m}+O(1)_{k_{m} \rightarrow+\infty}=\frac{1}{30}+O(1)_{k_{m} \rightarrow+\infty} \geq \frac{k_{m}}{31} .
$$

In particular, we have $\varpi(k)>1$, so that the proof for $Z \geq Z_{*}$ in the case $\frac{4 k_{m}}{9}+1 \leq k \leq \frac{2 k_{m}}{3}$ is analogous to the case $0 \leq k \leq \frac{4 k_{m}}{9}$. Details are left to the reader.

\section{Highest Sobolev norm}

In this section we improve the bootstrap bound (4.38) on the highest unweighted Sobolev norm of $(\tilde{\rho}, \Psi)$. Specifically, for (see (4.23))

$$
\|\tilde{\rho}, \Psi\|_{k_{m}}^{2}=\sum_{j=0}^{k_{m}} \sum_{|\alpha|=j} \int \frac{b^{2}\left|\nabla \nabla^{\alpha} \tilde{\rho}\right|^{2}+(p-1) \rho_{D}^{p-2} \rho_{\mathrm{Tot}}\left(\nabla^{\alpha} \tilde{\rho}\right)^{2}+\rho_{\mathrm{Tot}}^{2}\left|\nabla \nabla^{\alpha} \Psi\right|^{2}}{\langle Z\rangle^{2\left(k_{m}-j\right)}}
$$

we will establish the following

Proposition 7.1 (Control of the highest Sobolev norm) For some small constant d dependent on the data,

$$
\|\tilde{\rho}, \Psi\|_{k_{m}}^{2} \leq\left\|(\tilde{\rho}, \Psi)\left(\tau_{0}\right)\right\|_{k_{m}}^{2}+\ell
$$


Proof of Proposition 7.1 This follows from the global unweighted quasilinear energy identity. We let

$$
k_{m}=2 K_{m}, \quad K_{m} \in \mathbb{N}
$$

and denote in this section

$$
k=k_{m}, \quad \tilde{\rho}^{(k)}=\Delta^{K_{m}} \tilde{\rho}, \quad \Psi^{(k)}=\Delta^{K_{m}} \Psi .
$$

We recall the notation (5.11)

$$
I_{k_{m}}=\int b^{2}\left|\nabla \partial^{k_{m}} \tilde{\rho}\right|^{2}+(p-1) \int \rho_{D}^{p-2} \rho_{\mathrm{Tot}}\left|\partial^{k_{m}} \tilde{\rho}\right|^{2}+\int \rho_{\mathrm{Tot}}^{2}\left|\nabla \partial^{k_{m}} \Psi\right|^{2}
$$

Step 1 Control of lower order terms. We recall the notation:

$$
\mid \begin{aligned}
& \|\tilde{\rho}, \Psi\|_{k_{m}, \sigma(m)}^{2}=\sum_{j=0}^{k_{m}} \int \chi_{j, k_{m}, \sigma(m)} b^{2}\left|\nabla \partial^{j} \tilde{\rho}\right|^{2} \\
& \quad+(p-1) \int \chi_{j, k_{m}} \rho_{D}^{p-2} \rho_{\mathrm{Tot}}\left(\partial^{j} \tilde{\rho}\right)^{2}+\int \chi_{j, k_{m}} \rho_{\mathrm{Tot}}^{2}\left|\nabla \partial^{j} \Psi\right|^{2} \\
& \chi_{j, k_{m}, \sigma(m)}(Z)=\frac{1}{\langle Z\rangle^{2\left(k_{m}-j\right)}} .
\end{aligned}
$$

In view of (5.16) and (6.2), we have

$$
\|\tilde{\rho}, \Psi\|_{k_{m}, \sigma(m)}^{2} \leq \sum_{k=0}^{k_{m}} I_{k} \leq I_{k_{m}}+d_{0} .
$$

By Remark 4.2 we can replace (up to the lower order terms controlled as above) $I_{k_{m}}$ with

$$
\begin{aligned}
J_{k_{m}}:= & \int b^{2}\left|\nabla \Delta^{K_{m}} \tilde{\rho}\right|^{2}+(p-1) \int \rho_{D}^{p-2} \rho_{\mathrm{Tot}}\left|\Delta^{K_{m}} \tilde{\rho}\right|^{2} \\
& +\int \rho_{\mathrm{Tot}}^{2}\left|\nabla \Delta^{K_{m}} \Psi\right|^{2} .
\end{aligned}
$$

We claim: there exist $k_{m}^{*}(d, r, p), c_{d, r, p}>0$ such that for all $k_{m}>k_{m}^{*}(d, r, p)$, there holds:

$$
\frac{d}{d \tau}\left\{J_{k_{m}}[1+O(\delta)]\right\}+c_{d, r} k_{m} J_{k_{m}} \leq \ell .
$$

Integrating the above in time, using (4.24), (7.4), yields (7.2). 
Step 2 Energy identity. We revisit the computation of (5.6), (5.7), (5.8), (5.3) in order to extract all the coupling terms at the highest level of derivatives. Recall (5.3):

$$
\mid \begin{gathered}
\partial_{\tau} \tilde{\rho}=-\rho_{\mathrm{Tot}} \Delta \Psi-2 \nabla \rho_{\mathrm{Tot}} \cdot \nabla \Psi+H_{1} \tilde{\rho}-H_{2} \Lambda \tilde{\rho}-\tilde{\mathscr{E}}_{P, \rho} \\
\partial_{\tau} \Psi=b^{2} \frac{\Delta \rho_{\mathrm{Tot}}}{\rho_{\mathrm{Tot}}}-\left\{H_{2} \Lambda \Psi+\mu(r-2) \Psi\right. \\
\left.\quad+|\nabla \Psi|^{2}+(p-1) \rho_{D}^{p-2} \tilde{\rho}+\operatorname{NL}(\tilde{\rho})\right\}-\tilde{\mathscr{E}}_{P, \Psi}
\end{gathered}
$$

We use

$$
\left[\Delta^{K_{m}}, \Lambda\right]=k_{m} \Delta^{K_{m}}
$$

and recall (C.1):

$$
\left[\Delta^{k}, V\right] \Phi-2 k \nabla V \cdot \nabla \Delta^{k-1} \Phi=\sum_{|\alpha|+|\beta|=2 k,|\beta| \leq 2 k-2} c_{k, \alpha, \beta} \partial^{\alpha} V \partial^{\beta} \Phi,
$$

which gives

$$
\Delta^{K_{m}}\left(H_{2} \Lambda \tilde{\rho}\right)=k_{m}\left(H_{2}+\Lambda H_{2}\right) \rho_{k}+H_{2} \Lambda \rho_{k}+\mathcal{A}_{k}(\tilde{\rho})
$$

with

$$
\mid \begin{aligned}
& \left|\mathcal{A}_{k}(\tilde{\rho})\right| \lesssim c_{k} \sum_{j=1}^{k-1} \frac{\left|\nabla^{j} \tilde{\rho}\right|}{\langle Z\rangle^{k_{m}+r-j}}, \\
& \left|\nabla \mathcal{A}_{k}(\tilde{\rho})\right| \lesssim c_{k} \sum_{j=1}^{k} \frac{\left|\nabla^{j} \tilde{\rho}\right|}{\langle Z\rangle^{k_{m}+r+1-j}},
\end{aligned}
$$

where $\nabla^{j}=\partial_{1}^{\alpha_{1}} \cdots \partial_{d}^{\alpha_{d}}, j=\alpha_{1}+\cdots+\alpha_{d}$ denotes a generic derivative of order $j$. Using (C.1) again:

$$
\begin{aligned}
\partial_{\tau} \tilde{\rho}^{(k)}= & \left(H_{1}-k\left(H_{2}+\Lambda H_{2}\right)\right) \tilde{\rho}_{k}-H_{2} \Lambda \tilde{\rho}_{k}-\left(\Delta^{K_{m}} \rho_{\mathrm{Tot}}\right) \Delta \Psi \\
& -k \nabla \rho_{\mathrm{Tot}} \cdot \nabla \Psi^{(k)}-\rho_{\mathrm{Tot}} \Delta \Psi_{k} \\
& -2 \nabla\left(\Delta^{K_{m}} \rho_{\mathrm{Tot}}\right) \cdot \nabla \Psi-2 \nabla \rho_{\mathrm{Tot}} \cdot \nabla \Psi_{k}+F_{1}
\end{aligned}
$$

with

$$
\begin{aligned}
& F_{1}=-\Delta^{K_{m}} \tilde{\mathscr{E}}_{P, \rho}+\left[\Delta^{K_{m}}, H_{1}\right] \tilde{\rho}-A_{k}(\tilde{\rho})
\end{aligned}
$$

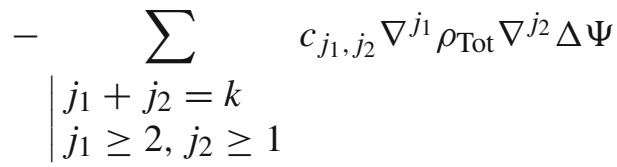




$$
-\sum_{\substack{j_{1}+j_{2}=k \\ j_{1}, j_{2} \geq 1}} c_{j_{1}, j_{2}} \nabla^{j_{1}} \nabla \rho_{\mathrm{Tot}} \cdot \nabla^{j_{2}} \nabla \Psi .
$$

For the second equation, we have similarly:

$$
\begin{aligned}
\partial_{\tau} \Psi_{k}= & b^{2}\left(\frac{\Delta^{K_{m}+1} \rho_{\mathrm{Tot}}}{\rho_{\mathrm{Tot}}}-\frac{k \nabla \Delta^{K_{m}} \rho_{\mathrm{Tot}} \cdot \nabla \rho_{\mathrm{Tot}}}{\rho_{\mathrm{Tot}}^{2}}\right) \\
& -k\left(H_{2}+\Lambda H_{2}\right) \Psi_{k}-H_{2} \Lambda \Psi_{k}-\mu(r-2) \Psi_{k}-2 \nabla \Psi \cdot \nabla \Psi_{k} \\
& -\left[(p-1) \rho_{P}^{p-2} \tilde{\rho}_{k}+k(p-1)(p-2) \rho_{D}^{p-3} \nabla \rho_{D} \cdot \nabla \Delta^{K_{m}-1} \tilde{\rho}\right] \\
& +F_{2}
\end{aligned}
$$

with

$$
\begin{aligned}
F_{2}= & -\partial^{k} \tilde{\mathscr{E}}_{P, \Psi}+b^{2}\left[\Delta^{K_{m}}\left(\frac{\Delta \rho_{\mathrm{Tot}}}{\rho_{\mathrm{Tot}}}\right)-\frac{\Delta^{K_{m}+1} \rho_{\mathrm{Tot}}}{\rho_{\mathrm{Tot}}}+\frac{k \nabla \Delta^{K_{m}} \rho_{\mathrm{Tot}} \cdot \nabla \rho_{\mathrm{Tot}}}{\rho_{\mathrm{Tot}}^{2}}\right] \\
& -(p-1)\left(\left[\Delta^{K_{m}}, \rho_{D}^{p-2}\right] \tilde{\rho}-k(p-2) \rho_{D}^{p-3} \nabla \rho_{D} \cdot \nabla \Delta^{K_{m}-1} \tilde{\rho}\right) \\
& -\mathcal{A}_{k}(\Psi)-\sum_{j_{1}+j_{2}=k, j_{1}, j_{2} \geq 1} \nabla^{j_{1}} \nabla \Psi \cdot \nabla^{j_{2}} \nabla \Psi-\Delta^{K_{m}} \mathrm{NL}(\tilde{\rho})
\end{aligned}
$$

and

$$
\mid \begin{aligned}
& \left|\mathcal{A}_{k}(\Psi)\right| \lesssim \sum_{j=1}^{k-1} \frac{\left|\nabla^{j} \Psi\right|}{\langle Z\rangle^{k_{m}+r-j}} \\
& \left|\nabla \mathcal{A}_{k}(\Psi)\right| \lesssim \sum_{j=1}^{k} \frac{\left|\nabla^{j} \Psi\right|}{\langle Z\rangle^{k_{m}+r+1-j}}
\end{aligned}
$$

We then run the global quasilinear energy identity similar to (5.10) with $\chi=1$ and obtain:

$$
\begin{aligned}
\frac{1}{2} \frac{d}{d \tau}\left\{\int b^{2}\left|\nabla \tilde{\rho}_{k}\right|^{2}+(p-1) \int \rho_{D}^{p-2} \rho_{\mathrm{Tot}} \tilde{\rho}_{k}^{2}+\int \rho_{\mathrm{Tot}}^{2}\left|\nabla \Psi_{k}\right|^{2}\right\} \\
=-\mu(r-2) b^{2} \int\left|\nabla \tilde{\rho}_{k}\right|^{2}+\int \partial_{\tau} \rho_{\mathrm{Tot}}\left[\frac{p-1}{2} \rho_{D}^{p-2} \tilde{\rho}_{k}^{2}+\rho_{\mathrm{Tot}}\left|\nabla \Psi_{k}\right|^{2}\right] \\
\quad+\frac{p-1}{2} \int(p-2) \partial_{\tau} \rho_{D} \rho_{D}^{p-3} \rho_{\mathrm{Tot}} \tilde{\rho}_{k}^{2} \\
\quad+\int F_{1}(p-1) \rho_{D}^{p-2} \rho_{\mathrm{Tot}} \tilde{\rho}_{k}+b^{2} \int \nabla F_{1} \cdot \nabla \tilde{\rho}_{k}+\int \rho_{T}^{2} \nabla F_{2} \cdot \nabla \Psi_{k} \\
\quad-\int k \nabla \rho_{\mathrm{Tot}} \cdot \nabla \Psi_{k}\left(-b^{2} \Delta \tilde{\rho}_{k}+(p-1) \rho_{D}^{p-2} \rho_{\mathrm{Tot}} \tilde{\rho}_{k}\right)
\end{aligned}
$$




$$
\begin{aligned}
& +\int b^{2} \frac{k \nabla \Delta^{K_{m}} \rho_{\mathrm{Tot}} \cdot \nabla \rho_{\mathrm{Tot}}}{\rho_{\mathrm{Tot}}}\left(\rho_{\mathrm{Tot}} \Delta \Psi_{k}+2 \nabla \rho_{\mathrm{Tot}} \cdot \nabla \Psi_{k}\right) \\
& +\int\left[\left(H_{1}-k\left(H_{2}+\Lambda H_{2}\right)\right) \tilde{\rho}_{k}-H_{2} \Lambda \tilde{\rho}_{k}\right. \\
& \left.-\left(\Delta^{K_{m}} \rho_{\mathrm{Tot}}\right) \Delta \Psi-2 \nabla\left(\Delta^{K_{m}} \rho_{\mathrm{Tot}}\right) \cdot \nabla \Psi\right] \\
& \times\left[-b^{2} \Delta \tilde{\rho}_{k}+(p-1) \rho_{D}^{p-2} \rho_{\mathrm{Tot}} \tilde{\rho}_{k}\right] \\
& -\int\left[b^{2} \rho_{\mathrm{Tot}} \Delta^{K_{m}+1} \rho_{D}-k \rho_{\mathrm{Tot}}\left(H_{2}+\Lambda H_{2}\right) \Psi_{k}\right. \\
& \left.-\rho_{\mathrm{Tot}} H_{2} \Lambda \Psi_{k}-\mu(r-2) \rho_{\mathrm{Tot}} \Psi_{k}-2 \rho_{\mathrm{Tot}} \nabla \Psi \cdot \nabla \Psi_{k}\right] \\
& \times\left[2 \nabla \rho_{\mathrm{Tot}} \cdot \nabla \Psi_{k}+\rho_{\mathrm{Tot}} \Delta \Psi_{k}\right]+\int k(p-1)(p-2) \rho_{\mathrm{Tot}} \rho_{D}^{p-3} \nabla \rho_{D} \\
& \cdot \nabla \Delta^{K_{m}-1} \tilde{\rho}\left[2 \nabla \rho_{\mathrm{Tot}} \cdot \nabla \Psi_{k}+\rho_{\mathrm{Tot}} \Delta \Psi_{k}\right] .
\end{aligned}
$$

We now estimate all terms in (7.13). The proof is similar to that one of Proposition 5.2 with two main differences: the absence of a cut-off function $\chi$, and a priori control of lower order derivatives from (7.4). The challenge here is to avoid any loss of derivatives and to compute exactly the quadratic form at the highest level of derivatives. The latter will be shown to be positive on a compact set in $Z$ provided $k_{m}>k_{m}^{*}(d, r, p) \gg 1$ has been chosen large enough.

In what follows, below, we will use $\delta>0$ as a small universal constant and will assume that the pointwise bounds (6.1) obtained on the lower order derivatives of $\tilde{\rho}$ and $\Psi$ are dominated by $\delta$. On the set $Z \leq Z^{*}$, this will often be a source of smallness, while for $Z \geq Z^{*}$, we may use the bootstrap bounds (4.40) and the $\delta$-smallness will be generated by extra powers of $Z$. We also note that from (7.6) the quadratic form is expected to be proportionate to $k_{m} I_{k_{m}}$. Choosing $k_{m}$ large will allow us to dominate other quadratic terms without smallness but with the uniform dependence on $k_{m}$. The notation $\lesssim$ will allow dependence on $k_{m}$, while $O$ will indicate a bound independent of $k_{m}$. As before, $\ell_{0}$ (as well as $\ell$ ) will denote small constants, dependent on the data (or, more precisely, on $\tau_{0}$ ), that can be made arbitrarily small. In particular, we will use

$$
\|\tilde{\rho}, \Psi\|_{k_{m}-1, \sigma\left(k_{m}-1\right)} \leq \ell_{0} .
$$

The constants $\delta \gg d_{0}$ will be assumed to be smaller than any power of $k_{m}$, so that our calculations will be unaffected by combinatorics generated by taking $k_{m}$ derivatives of the equations.

Step 3 Leading order terms. 
Cross term. Recall (5.27):

$$
\begin{aligned}
\int \Delta g F \cdot \nabla g d x & =\sum_{i, j=1}^{d} \int \partial_{i}^{2} g F_{j} \partial_{j} g d x \\
& =-\sum_{i, j=1}^{d} \int \partial_{i} g\left(\partial_{i} F_{j} \partial_{j} g+F_{j} \partial_{i, j}^{2} g\right) \\
& =-\sum_{i, j=1}^{d} \int \partial_{i} F_{j} \partial_{i} g \partial_{j} g+\frac{1}{2} \int|\nabla g|^{2} \nabla \cdot F .
\end{aligned}
$$

Letting $g=g_{1}+g_{2}$ yields a bilinear off-diagonal Pohozhaev identity:

$$
\begin{aligned}
\int & {\left[\Delta g_{1} F \cdot \nabla g_{2}+\Delta g_{2} F \cdot \nabla g_{1}\right] d x } \\
= & -\sum_{i, j=1}^{d} \int \partial_{i} F_{j}\left(\partial_{i} g_{1} \partial_{j} g_{2}+\partial_{i} g_{2} \partial_{j} g_{1}\right) \\
& +\int \nabla g_{1} \cdot \nabla g_{2}(\nabla \cdot F)
\end{aligned}
$$

We may therefore integrate by parts the one term in (7.13) which has too many derivatives:

$$
\begin{aligned}
& b^{2} k\left|\int\left[\nabla \rho_{\mathrm{Tot}} \cdot \nabla \Psi_{k} \Delta \tilde{\rho}_{k}+\nabla \Delta^{K_{m}} \rho_{\mathrm{Tot}} \cdot \nabla \rho_{\mathrm{Tot}} \Delta \Psi_{k}\right]\right| \\
& =b^{2} k\left|\int \nabla \rho_{\mathrm{Tot}} \cdot \nabla \Psi_{k} \Delta \tilde{\rho}_{k}+\nabla \rho_{\mathrm{Tot}} \cdot \nabla \tilde{\rho}_{k} \Delta \Psi_{k}+\nabla \rho_{\mathrm{Tot}} \cdot \nabla \Delta^{K_{m}} \rho_{D} \Delta \Psi_{k}\right| \\
& =b^{2} k \mid-\int \sum_{i, j=1}^{d} \partial_{i, j}^{2} \rho_{\mathrm{Tot}}\left(\partial_{i} \tilde{\rho}_{k} \partial_{j} \Psi_{k}+\partial_{i} \Psi_{k} \partial_{j} \tilde{\rho}_{k}\right)+\int \nabla \tilde{\rho}_{k} \cdot \nabla \Psi_{k} \Delta \rho_{\mathrm{Tot}} \\
& \quad-\int \nabla \Psi_{k} \cdot \nabla\left(\nabla \rho_{\mathrm{Tot}} \cdot \nabla \Delta^{K_{m}} \rho_{D}\right) \mid \\
& \lesssim b^{2} k \int \rho_{\mathrm{Tot}}\left|\nabla \Psi_{k}\right|\left[\frac{1}{\langle Z\rangle^{k+2}}+\frac{\left|\nabla \tilde{\rho}_{k}\right|}{\langle Z\rangle^{2}}\right] \\
& \leq \delta \int \rho_{\mathrm{Tot}}^{2}\left|\nabla \Psi_{k}\right|^{2}+C_{\delta} b^{4}+C_{\delta} b^{4} \int\left|\nabla \tilde{\rho}_{k}\right|^{2} \\
& \leq \delta J_{k_{m}}+C_{\delta} b^{4} .
\end{aligned}
$$


We estimate similarly:

$$
\begin{aligned}
& \left|k b^{2} \int \frac{\nabla \Delta^{K_{m}} \rho_{\mathrm{Tot}} \cdot \nabla \rho \mathrm{Tot}}{\rho_{\mathrm{Tot}}} \nabla \rho_{\mathrm{Tot}} \cdot \nabla \Psi_{k}\right| \\
& \quad \leq \delta\left[b^{2} \int\left|\nabla \tilde{\rho}_{k}\right|^{2}+\int \rho_{\mathrm{Tot}}^{2}\left|\nabla \Psi_{k}\right|^{2}\right]+c_{\delta} b^{4} \lesssim \delta J_{k_{m}}+C_{\delta} b^{4} .
\end{aligned}
$$

We use

$$
\frac{|\tilde{\rho}|}{\rho_{\mathrm{Tot}}}+\frac{|\Lambda \tilde{\rho}|}{\langle Z\rangle^{c} \rho_{\mathrm{Tot}}} \lesssim \delta, \quad 0<c \ll 1
$$

to compute the first coupling term:

$$
\begin{aligned}
& -k(p-1) \int \nabla \rho_{\mathrm{Tot}} \cdot \nabla \Psi_{k} \rho_{D}^{p-2} \rho_{\mathrm{Tot}} \tilde{\rho}_{k} \\
& =-k \int \rho_{D} \nabla \rho_{D}^{p-1} \cdot \nabla \Psi_{k} \tilde{\rho}_{k}+O\left(\delta \int \frac{\left|\nabla \Psi_{k}\right| \rho_{D}^{p-1} \rho_{\mathrm{Tot}}\left|\tilde{\rho}_{k}\right|}{\langle Z\rangle}\right) \\
& =-k \int \rho_{D} \nabla \rho_{D}^{p-1} \cdot \nabla \Psi_{k} \tilde{\rho}_{k}+O\left(\delta J_{k_{m}}\right) .
\end{aligned}
$$

The second coupling term is computed after an integration by parts using (7.15), the control of lower order terms (7.4) and the spherically symmetric assumption:

$$
\begin{aligned}
\int & \left(\rho_{\mathrm{Tot}} \Delta \Psi_{k}+2 \nabla \rho_{\mathrm{Tot}} \cdot \nabla \Psi_{k}\right) k(p-1)(p-2) \rho_{\mathrm{Tot}} \rho_{D}^{p-3} \nabla \rho_{D} \cdot \nabla \Delta^{K_{m}-1} \tilde{\rho} \\
= & k(p-1)(p-2) \int \nabla \cdot\left(\rho_{\mathrm{Tot}}^{2} \nabla \Psi_{k}\right) \rho_{D}^{p-3} \nabla \rho_{D} \cdot \nabla \Delta^{K_{m}-1} \tilde{\rho} \\
= & -k(p-1)(p-2) \int \rho_{\mathrm{Tot}}^{2} \nabla \Psi_{k} \cdot \nabla\left(\rho_{D}^{p-3} \nabla \rho_{D} \cdot \nabla \Delta^{K_{m}-1} \tilde{\rho}\right) \\
= & -k(p-1)(p-2) \int \rho_{\mathrm{Tot}}^{2} \partial_{Z} \Psi_{k} \partial_{Z}\left(\rho_{D}^{p-3} \partial_{Z} \rho_{D} \partial_{Z} \Delta^{K_{m}-1} \tilde{\rho}\right) \\
= & -k(p-1)(p-2) \int \rho_{D}^{p-3} \partial_{Z} \rho_{D} \rho_{\mathrm{Tot}}^{2} \partial_{Z} \Psi_{k} \partial_{Z}^{2} \Delta^{K_{m}-1} \tilde{\rho} \\
& +O\left(\int \rho_{\mathrm{Tot}}\left|\nabla \Psi_{k}\right| \rho_{\mathrm{Tot}}^{p-1} \frac{\left|\nabla^{k_{m}-1} \tilde{\rho}\right|}{\langle Z\rangle^{2}}\right) \\
= & -\int k(p-2) \rho_{D} \partial_{Z}\left(\rho_{D}^{p-1}\right) \partial_{Z} \Psi_{k} \tilde{\rho}_{k} \\
& +\int k(p-2)(d-1) \rho_{D} \partial_{Z}\left(\rho_{D}^{p-1}\right) \partial_{Z} \Psi_{k} \frac{\partial_{Z} \Delta^{K_{m}-1} \tilde{\rho}}{|Z|}
\end{aligned}
$$




$$
\begin{aligned}
& +O\left(\int \rho_{\mathrm{Tot}}\left|\nabla \Psi_{k}\right| \rho_{\mathrm{Tot}}^{p-1} \frac{\left|\nabla^{k_{m}-1} \tilde{\rho}\right|}{\langle Z\rangle^{2}}\right) \\
= & -k(p-2) \int \rho_{D} \nabla \rho_{D}^{p-1} \cdot \nabla \Psi_{k} \tilde{\rho}_{k}+O\left(d_{0}+\delta J_{k_{m}}\right),
\end{aligned}
$$

where in the last step we used that $\frac{\left|\partial_{Z} \rho_{D}\right|}{|Z| \rho_{D}} \lesssim \frac{1}{\langle Z\rangle^{2}}$.

$\rho_{k}$ terms. We compute:

$$
\begin{aligned}
\int & \left(H_{1}-k\left(H_{2}+\Lambda H_{2}\right)\right) \tilde{\rho}_{k}\left(-b^{2} \Delta \tilde{\rho}_{k}+(p-1) \rho_{D}^{p-2} \rho_{\operatorname{Tot}} \tilde{\rho}_{k}\right) \\
= & \int\left(H_{1}-k\left(H_{2}+\Lambda H_{2}\right)\right)\left[b^{2}\left|\nabla \tilde{\rho}_{k}\right|^{2}+(p-1) \rho_{D}^{p-2} \rho_{\mathrm{Tot}} \tilde{\rho}_{k}^{2}\right] \\
& -\frac{b^{2}}{2} \int \tilde{\rho}_{k}^{2} \Delta\left(H_{1}-k\left(H_{2}+\Lambda H_{2}\right)\right) .
\end{aligned}
$$

We now use the global lower bound, see properties (2.21) and (2.22) of the the profile $(w, \sigma)$,

$$
H_{2}+\Lambda H_{2}=\mu(1-w-\Lambda w) \geq c_{p, d, r}, \quad c_{p, d, r}>0
$$

to estimate using (8.17), (7.14):

$$
\begin{aligned}
\int\left(H_{1}-k\left(H_{2}+\Lambda H_{2}\right)\right) \tilde{\rho}_{k}\left(-b^{2} \Delta \tilde{\rho}_{k}+(p-1) \rho_{D}^{p-2} \rho_{\mathrm{Tot}} \tilde{\rho}_{k}\right) \\
\leq-k \int\left[1+O_{k_{m} \rightarrow+\infty}\left(\frac{1}{k_{m}}\right)\right]\left(H_{2}+\Lambda H_{2}\right) \\
\quad \times\left[b^{2}\left|\nabla \tilde{\rho}_{k}\right|^{2}+(p-1) \rho_{D}^{p-2} \rho_{\mathrm{Tot}} \tilde{\rho}_{k}^{2}\right]+C b^{2} \int \frac{\tilde{\rho}_{k}^{2}}{\langle Z\rangle^{2+r}} \\
\leq-k \int\left(H_{2}+\Lambda H_{2}\right)\left[b^{2}\left|\nabla \tilde{\rho}_{k}\right|^{2}+(p-1) \rho_{D}^{p-2} \rho_{\mathrm{Tot}} \tilde{\rho}_{k}^{2}\right]+d_{0} .
\end{aligned}
$$

Next, using

$$
\left|\partial^{k} \rho_{D}\right| \lesssim \frac{\rho_{D}}{\langle Z\rangle^{k}}
$$

we estimate from (4.40):

$$
\begin{aligned}
& b^{2}\left|\int \Delta \tilde{\rho}_{k}\left[\left(\Delta^{K_{m}} \rho_{D}\right) \Delta \Psi+2 \nabla\left(\Delta^{K_{m}} \rho_{D}\right) \cdot \nabla \Psi\right]\right| \\
& \quad \lesssim b^{2} \int\left|\nabla \tilde{\rho}_{k}\right|\left[\left|\nabla\left(\Delta^{K_{m}} \rho_{D} \Delta \Psi\right)\right|+\left|\nabla\left(\nabla \Delta^{K_{m}} \rho_{D} \cdot \nabla \Psi\right)\right|\right]
\end{aligned}
$$




$$
\leq b^{2} \delta \int\left|\nabla \tilde{\rho}_{k}\right|^{2}+\frac{b^{2}}{\delta} \sum_{j=1}^{3} \int \frac{\rho_{D}^{2}\left|\partial^{j} \Psi\right|^{2}}{\langle Z\rangle^{2(k+3-j)}} \leq \delta J_{k_{m}}+d_{0} b^{2} .
$$

For the nonlinear term, we use (6.1), (4.40), (5.27), (7.4) to estimate

$$
\begin{aligned}
& b^{2}\left|\int \Delta \tilde{\rho}_{k}\left[\tilde{\rho}_{k} \Delta \Psi+2 \nabla \tilde{\rho}_{k} \cdot \nabla \Psi\right]\right| \\
& \quad \lesssim b^{2}\left[\int\left|\partial^{2} \Psi\right|\left|\nabla \tilde{\rho}_{k}\right|^{2}+\int \frac{\tilde{\rho}_{k}^{2}}{\langle Z\rangle^{2}}\right] \leq \delta J_{k_{m}}+d_{0} b^{2} .
\end{aligned}
$$

Next

$$
\begin{aligned}
& \left|\int\left[\left(\Delta^{K_{m}} \rho_{D}\right) \Delta \Psi-2 \nabla\left(\Delta^{K_{m}} \rho_{D}\right) \cdot \nabla \Psi\right](p-1) \rho_{D}^{p-2} \rho_{\mathrm{Tot}} \tilde{\rho}_{k}\right| \\
& \quad \leq \delta \int \rho_{D}^{p-2} \rho_{\mathrm{Tot}} \tilde{\rho}_{k}^{2}+\frac{C}{\delta} \int \rho_{D}^{p-2} \rho_{\mathrm{Tot}}^{2}\left[\frac{\left|\partial^{2} \Psi\right|^{2}}{\langle Z\rangle^{2 k}}+\frac{|\partial \Psi|^{2}}{\langle Z\rangle^{2(k+1)}}\right] \\
& \quad \leq \delta J_{k_{m}}+d_{0},
\end{aligned}
$$

since we are assuming that $d_{0} \ll \delta$, and for the nonlinear term after an integration by parts:

$$
\left|\int\left[\tilde{\rho}_{k} \Delta \Psi-2 \nabla \tilde{\rho}_{k} \cdot \nabla \Psi\right](p-1) \rho_{D}^{p-2} \rho_{\operatorname{Tot}} \tilde{\rho}_{k}\right| \lesssim \delta \int \rho_{D}^{p-2} \rho_{\operatorname{Tot}} \tilde{\rho}_{k}^{2} .
$$

From Pohozhaev (5.27):

$$
-\int H_{2} \Lambda \tilde{\rho}_{k}\left(-b^{2} \Delta \tilde{\rho}_{k}\right)=b^{2} \int \Delta \tilde{\rho}_{k}\left(Z H_{2}\right) \cdot \nabla \tilde{\rho}_{k}=O\left(b^{2} \int\left|\nabla \tilde{\rho}_{k}\right|^{2}\right) .
$$

Integrating by parts and using (8.17), (5.41), (5.42):

$$
\begin{aligned}
- & \int H_{2} \Lambda \tilde{\rho}_{k}\left[(p-1) \rho_{D}^{p-2} \rho_{\mathrm{Tot}} \tilde{\rho}_{k}\right] \\
& +\frac{p-1}{2} \int(p-2) \partial_{\tau} \rho_{D} \rho_{D}^{p-3} \rho_{\mathrm{Tot}} \tilde{\rho}_{k}^{2}+\frac{p-1}{2} \int \partial_{\tau} \rho_{\mathrm{Tot}} \rho_{D}^{p-2} \tilde{\rho}_{k}^{2} \\
= & \frac{p-1}{2} \int \tilde{\rho}_{k}^{2}\left[\nabla \cdot\left(Z H_{2} \rho_{D}^{p-2} \rho_{\mathrm{Tot}}\right)+\partial_{\tau}\left(\rho_{D}^{p-2}\right) \rho_{\mathrm{Tot}}+\partial_{\tau} \rho_{\mathrm{Tot}} \rho_{D}^{p-2}\right] \\
= & O\left(\int \rho_{D}^{p-2} \rho_{\mathrm{Tot}} \tilde{\rho}_{k}^{2}\right)
\end{aligned}
$$


Note that the above two bounds, even though dependent on the highest order derivatives, contain no $k$ dependence.

$\Psi_{k}$ terms. After an integration by parts:

$$
\begin{aligned}
& \left|\int b^{2} \Delta^{K_{m}+1} \rho_{D}\left[2 \nabla \rho_{\mathrm{Tot}} \cdot \nabla \Psi_{k}+\rho_{\mathrm{Tot}} \Delta \Psi_{k}\right]\right| \\
& \quad \lesssim b^{2} \int \rho_{\mathrm{Tot}} \frac{\left|\nabla \Psi_{k}\right|}{\langle Z\rangle^{k+3}} \leq \delta \int \rho_{\mathrm{Tot}}^{2}\left|\nabla \Psi_{k}\right|^{2}+\alpha_{0} .
\end{aligned}
$$

Then

$$
\begin{aligned}
& \mu(r-2) \int \rho_{\mathrm{Tot}} \Psi_{k}\left[2 \nabla \rho_{\mathrm{Tot}} \cdot \nabla \Psi_{k}+\rho_{\mathrm{Tot}} \Delta \Psi_{k}\right] \\
& =-\mu(r-2) \int \Psi_{k}^{2} \nabla \cdot\left(\rho_{\mathrm{Tot}} \nabla \rho_{\mathrm{Tot}}\right)-\mu(r-2) \int \nabla \Psi_{k} \cdot \nabla\left(\rho_{\mathrm{Tot}}^{2} \Psi_{k}\right) \\
& =-\mu(r-2) \int \rho_{\mathrm{Tot}}^{2}\left|\nabla \Psi_{k}\right|^{2}
\end{aligned}
$$

and similarly, using (8.17), (7.4):

$$
\begin{aligned}
k & \int \rho_{\mathrm{Tot}}\left(H_{2}+\Lambda H_{2}\right) \Psi_{k}\left[2 \nabla \rho_{\mathrm{Tot}} \cdot \nabla \Psi_{k}+\rho_{\mathrm{Tot}} \Delta \Psi_{k}\right] \\
= & k \int\left(H_{2}+\Lambda H_{2}\right) \Psi_{k} \nabla \cdot\left(\rho_{\mathrm{Tot}}^{2} \nabla \Psi_{k}\right) \\
= & -k\left[\int\left(H_{2}+\Lambda H_{2}\right) \rho_{\mathrm{Tot}}^{2}\left|\nabla \Psi_{k}\right|^{2}\right. \\
& \left.+\int \rho_{\mathrm{Tot}}^{2} \Psi_{k}^{2}\left(\frac{\nabla \cdot\left(\rho_{\mathrm{Tot}}^{2} \nabla\left(H_{2}+\Lambda H_{2}\right)\right)}{2 \rho_{T}^{2}}\right)\right] \\
= & -k \int\left(H_{2}+\Lambda H_{2}\right) \rho_{\mathrm{Tot}}^{2}\left|\nabla \Psi_{k}\right|^{2}+d_{0},
\end{aligned}
$$

where the $\Psi_{k}^{2}$ term is controlled, with the help of the bound

$$
\left|\frac{\nabla \cdot\left(\rho_{\operatorname{Tot}}^{2} \nabla\left(H_{2}+\Lambda H_{2}\right)\right)}{2 \rho_{T}^{2}}\right| \lesssim\langle Z\rangle^{-2-r},
$$

by using the already bounded $\|(\tilde{\rho}, \Psi)\|_{k_{m}-1, \sigma\left(k_{m}-1\right)}$-norm.

Then, from (6.1) and (6.9):

$$
\left|\int 2 \rho_{\mathrm{Tot}} \nabla \Psi \cdot \nabla \Psi_{k}\left(2 \nabla \rho_{\mathrm{Tot}} \cdot \nabla \Psi_{k}\right)\right| \lesssim \ell_{0} \int \rho_{\mathrm{Tot}}^{2}\left|\nabla \Psi_{k}\right|^{2}
$$


and using (5.27):

$$
\begin{aligned}
\left|\int 2 \rho_{\mathrm{Tot}} \nabla \Psi \cdot \nabla \Psi_{k}\left(\rho_{\mathrm{Tot}} \Delta \Psi_{k}\right)\right| & \lesssim \int\left|\nabla \Psi_{k}\right|^{2}|| \partial\left(\rho_{\mathrm{Tot}}^{2} \nabla \Psi\right) \mid \\
& \lesssim \mathcal{Q}_{0} \int \rho_{\mathrm{Tot}}^{2}\left|\nabla \Psi_{k}\right|^{2}
\end{aligned}
$$

Arguing verbatim as in the proof of (5.49) produces the bound

$$
\left|\int \rho_{\mathrm{Tot}} H_{2} \Lambda \Psi_{k}\left(2 \nabla \rho_{\mathrm{Tot}} \cdot \nabla \Psi_{k}+\rho_{\mathrm{Tot}} \Delta \Psi_{k}\right)\right|=O\left(\int \rho_{\mathrm{Tot}}^{2}\left|\nabla \Psi_{k}\right|^{2}\right) .
$$

Step $4 F_{1}$ terms. We claim the bound:

$$
b^{2} \int\left|\nabla F_{1}\right|^{2}+(p-1) \int \rho_{D}^{p-1} F_{1}^{2} \lesssim \delta J_{k_{m}}+d_{0} .
$$

Source term induced by localization. From (5.55), for $k_{m}$ large enough:

$$
\int \rho_{D}^{p-2} \rho_{\mathrm{Tot}}\left|\Delta^{K_{m}} \tilde{\mathscr{E}}_{P, \rho}\right|^{2}+b^{2} \int\left|\nabla \Delta^{K_{m}} \tilde{\mathscr{E}}_{P, \rho}\right|^{2} \lesssim d_{0}
$$

$\left[\Delta^{K_{m}}, H_{1}\right]$ term. We estimate from (5.56), (7.14)

$$
(p-1) \int \rho_{D}^{p-1}\left(\left[\Delta^{K_{m}}, H_{1}\right] \tilde{\rho}\right)^{2} \lesssim \sum_{j=0}^{k-1} \int \rho_{D}^{p-1} \frac{\left|\partial^{j} \tilde{\rho}\right|^{2}}{\langle Z\rangle^{2(r+k-j)}} \leq \iota_{0}
$$

and

$$
\begin{aligned}
& b^{2} \int\left|\nabla\left(\left[\Delta^{K_{m}}, H_{1}\right] \tilde{\rho}\right)\right|^{2} \lesssim b^{2} \sum_{j=0}^{k} \int \frac{\left|\partial_{j} \tilde{\rho}\right|^{2}}{\langle Z\rangle^{2(1+r+k-j)}} \\
& \quad=b^{2} \int \frac{\tilde{\rho}^{2} d Z}{\langle Z\rangle^{2(1+r+k)}}+b^{2} \sum_{j=0}^{k-1} \int \frac{\left|\partial^{j} \nabla \tilde{\rho}\right|^{2}}{\langle Z\rangle^{2(r+k+1-j)+2}} \\
& \quad \lesssim b^{2}+\|\tilde{\rho}, \Psi\|_{k_{m}-1, \sigma\left(k_{m}-1\right)} \leq d_{0} .
\end{aligned}
$$

$\mathcal{A}_{k}(\tilde{\rho})$ term. From (7.7), (7.14):

$$
(p-1) \int \rho_{D}^{p-1}\left(\mathcal{A}_{k}(\tilde{\rho})\right)^{2} \lesssim \sum_{j=1}^{k-1} \int \rho_{D}^{p-1} \frac{\left|\nabla^{j} \tilde{\rho}\right|^{2}}{\langle Z\rangle^{2(r+k-j)}} \leq d_{0}
$$


and

$$
b^{2} \int\left|\nabla\left(\mathcal{A}_{k}(\tilde{\rho})\right)\right|^{2} \lesssim b^{2} \sum_{j=0}^{k-1} \int \frac{\left|\nabla \nabla^{j} \tilde{\rho}\right|^{2}}{\langle Z\rangle^{2(r+k-j)}} \leq d_{0}
$$

and (7.16) is proved for this term.

Nonlinear term. After changing indices, we need to estimate

$$
N_{j_{1}, j_{2}}=\nabla^{j_{1}} \rho_{\mathrm{Tot}} \nabla^{j_{2}} \nabla \Psi, \quad j_{1}+j_{2}=k+1, \quad 2 \leq j_{1}, j_{2} \leq k-1 .
$$

For the profile term:

$$
\left|\partial^{j_{1}} \rho_{D} \nabla^{j_{2}} \nabla \Psi\right| \lesssim \rho_{D} \frac{\left|\nabla^{j_{2}} \nabla \Psi\right|}{\langle Z\rangle^{j_{1}}}=\rho_{D} \frac{\left|\nabla^{j_{2}} \nabla \Psi\right|}{\langle Z\rangle^{k+1-j_{2}}}
$$

and therefore, recalling (5.59), (7.14):

$$
\int(p-1) N_{j_{1}, j_{2}}^{2} \rho_{D}^{p-1} \lesssim \int \frac{\rho_{\mathrm{Tot}}^{2}\left|\nabla^{j_{2}} \nabla \Psi\right|^{2}}{\langle Z\rangle^{2\left(k+1-j_{2}\right)+2(r-1)}} \leq d_{0}
$$

Similarly, after taking a derivative:

$$
\begin{aligned}
b^{2} \int\left|\nabla N_{j_{1}, j_{2}}\right|^{2} & \lesssim b^{2} \int \frac{\rho_{\text {Tot }}^{2}\left|\nabla^{j_{2}} \nabla \Psi\right|^{2}}{\langle Z\rangle^{2\left(k+2-j_{2}\right)}}+b^{2} \int \frac{\rho_{\mathrm{Tot}}^{2}\left|\nabla j^{j_{2}+1} \nabla \Psi\right|^{2}}{\langle Z\rangle^{2\left(k+1-j_{2}\right)}} \\
& \leq d_{0}+\delta J_{k_{m}} .
\end{aligned}
$$

The $\delta J_{k_{m}}$ term above controls the case $j_{2}=k-1$.

We now turn to the control of the nonlinear term. If $j_{1} \leq \frac{4 k_{m}}{9}$, then from (4.40), (7.4):

$$
\int \rho_{D}^{p-1}\left|\nabla^{j_{1}} \tilde{\rho} \nabla^{j_{2}} \nabla \Psi\right|^{2} \lesssim \int \rho_{D}^{2} \frac{\left|\nabla^{j_{2}} \nabla \Psi\right|^{2}}{\langle Z\rangle^{2\left(k+1-j_{2}\right)+(p-1) \frac{2(r-1)}{p-1}}} \leq d_{0}
$$

If $j_{2} \leq \frac{4 k_{m}}{9}$, then from (4.40) with $b=\frac{1}{\left(Z^{*}\right)^{r-2}}$ :

$$
\begin{aligned}
\int \rho_{D}^{p-1}\left|\nabla^{j_{1}} \tilde{\rho} \nabla^{j_{2}} \nabla \Psi\right|^{2} \lesssim & \int_{Z \leq Z^{*}} \rho_{D}^{p-1} \frac{\left|\nabla^{j_{1}} \tilde{\rho}\right|^{2}}{\langle Z\rangle^{2\left(k+1+(r-2)-j_{1}\right)}} \\
& +b^{2} \int_{Z \geq Z^{*}} \rho_{D}^{p-1} \frac{\left|\nabla^{j_{1}} \tilde{\rho}\right|^{2}}{\langle Z\rangle^{2\left(k+1-j_{1}\right)}} \leq d_{0} .
\end{aligned}
$$


We may therefore assume $j_{1}, j_{2} \geq m_{0}=\frac{4 k_{m}}{9}+1$, which implies $k \geq m_{0}$ and $j_{1}, j_{2} \leq \frac{2 k_{m}}{3}$ and hence from (4.40) and (6.1):

$$
\int \rho_{D}^{p-1}\left|\partial^{j_{1}} \tilde{\rho} \nabla^{j_{2}} \nabla \Psi\right|^{2} \lesssim \iota_{0}+\int_{Z \geq Z^{*}} \frac{d Z}{\langle Z\rangle^{\frac{k_{m}}{10}}} \leq \iota_{0}
$$

The $b^{2}$ derivative contribution of the nonlinear term is estimated similarly.

Step $5 F_{2}$ terms. We claim:

$$
\int \rho_{\mathrm{Tot}}^{2}\left|\nabla\left(F_{2}+\Delta^{K_{m}} \mathrm{NL}(\tilde{\rho})\right)\right|^{2} \leq \delta J_{k_{m}}+\iota_{0}
$$

The nonlinear term $\Delta^{K_{m}} \mathrm{NL}(\tilde{\rho})$ will be treated in the next step. $\mathcal{A}_{k}(\Psi)$ term. From (7.12)

$$
\left|\nabla \mathcal{A}_{k}(\Psi)\right| \lesssim \sum_{j=1}^{k} \frac{\left|\nabla^{j} \Psi\right|}{\langle Z\rangle^{r+k-j+1}}
$$

and hence:

$$
\int \rho_{\mathrm{Tot}}^{2}\left|\nabla \mathcal{A}_{k}(\Psi)\right|^{2} \lesssim \sum_{j=0}^{k-1} \int \rho_{\mathrm{Tot}}^{2} \frac{\left|\nabla \nabla^{j} \Psi\right|^{2}}{\langle Z\rangle^{2(r+k-j)}} \leq d_{0}
$$

$\left[\Delta^{K_{m}}, \rho_{D}^{p-2}\right]$ term. From (C.1):

$$
\left|\left[\Delta^{K_{m}}, \rho_{D}^{p-2}\right] \tilde{\rho}-k(p-2) \rho_{D}^{p-3} \nabla \rho_{D} \cdot \nabla \Delta^{K_{m}-1} \tilde{\rho}\right| \lesssim \sum_{j=0}^{k-2} \frac{\left|\nabla^{j} \tilde{\rho}\right|}{\langle Z\rangle^{k-j}} \rho_{D}^{p-2}
$$

After taking a derivative:

$$
\begin{aligned}
& \int \rho_{\mathrm{Tot}}^{2}\left|\nabla\left[\left[\Delta^{K_{m}}, \rho_{D}^{p-2}\right] \tilde{\rho}-k(p-2) \rho_{D}^{p-3} \nabla \rho_{D} \cdot \nabla \Delta^{K_{m}-1} \tilde{\rho}\right]\right|^{2} \\
& \lesssim \sum_{j=0}^{k-1} \int \rho_{D}^{2(p-2)+2} \frac{\left|\nabla^{j} \tilde{\rho}\right|^{2}}{\langle Z\rangle^{2(k-j)+2}} \leq \iota_{0}
\end{aligned}
$$

Nonlinear $\Psi$ term. Let

$$
\partial N_{j_{1}, j_{2}}=\nabla^{j_{1}} \nabla \Psi \nabla^{j_{2}} \nabla \Psi, \quad j_{1}+j_{2}=k+1, \quad j_{1}, j_{2} \geq 1
$$


We first treat the highest derivative term using the $L^{\infty}$ smallness of small derivatives: Using (4.40) and (6.1)

$$
\int \rho_{\mathrm{Tot}}^{2}|\nabla \nabla \Psi|^{2}\left|\nabla^{k_{m}} \nabla \Psi\right|^{2} \leq\left(d_{0}+b^{2}\right) I_{k_{m}} .
$$

We now assume $j_{1}, j_{2} \leq k_{m}-1$. If $j_{1} \leq \frac{4 k_{m}}{9}$, then from (4.40), (7.4):

$$
\int \rho_{\mathrm{Tot}}^{2}\left|\nabla N_{j_{1}, j_{2}}\right|^{2} \lesssim\left(d_{0}+b^{2}\right) \int \rho_{\mathrm{Tot}}^{2} \frac{\left|\nabla^{j_{2}} \nabla \Psi\right|^{2}}{\langle Z\rangle^{2\left(k+1-j_{2}\right)}} \leq d_{0} .
$$

The expression being symmetric in $j_{1}, j_{2}$, we may assume $j_{1}, j_{2} \geq m_{0}=$ $\frac{4 k_{m}}{9}+1, j_{1}, j_{2} \leq \frac{2 k_{m}}{3}$, and using (4.40), (7.4):

$$
\int \rho_{\text {Tot }}^{2}\left|\nabla N_{j_{1}, j_{2}}\right|^{2} \lesssim \alpha_{0} \int_{Z \leq Z^{*}} \frac{d Z}{\langle Z\rangle^{\frac{k_{m}}{10}}}+b^{4} \int_{Z>Z^{*}} \frac{d Z}{\langle Z\rangle^{\frac{k_{m}}{10}}} \leq \iota_{0} .
$$

Quantum pressure term. We estimate from Leibniz and (C.1):

$$
\begin{aligned}
& b^{2}\left|\Delta^{K_{m}}\left(\frac{\Delta \rho_{\mathrm{Tot}}}{\rho_{\mathrm{Tot}}}\right)-\frac{\Delta^{K_{m}+1} \rho_{\mathrm{Tot}}}{\rho_{\mathrm{Tot}}}+\frac{k \nabla \Delta^{K_{m}} \rho_{\mathrm{Tot}} \cdot \nabla \rho_{\mathrm{Tot}}}{\rho_{\mathrm{Tot}}^{2}}\right| \\
& \lesssim_{k} b^{2} \sum_{j_{1}+j_{2}=k, j_{2} \geq 2}\left|\nabla^{j_{1}} \Delta \rho_{\mathrm{Tot}} \partial^{j_{2}}\left(\frac{1}{\rho_{\mathrm{Tot}}}\right)\right| .
\end{aligned}
$$

We use the Faa di Bruno formula:

$$
N_{j_{1}, j_{2}}=b^{2} \nabla^{j_{1}+1} \Delta \rho_{\mathrm{Tot}} \frac{1}{\rho_{\mathrm{Tot}}^{j_{2}+1}} \sum_{m_{1}+2 m_{2}+\cdots+j_{2} m_{j_{2}}=j_{2}} \Pi_{i=1}^{j_{2}}\left(\nabla^{i} \rho_{\mathrm{Tot}}\right)^{m_{i}}
$$

and $m_{1}+2 m_{2}+\cdots+j_{2} m_{j_{2}}=j_{2}$. We decompose $\rho_{\text {Tot }}=\rho_{D}+\tilde{\rho}$ in the sum and estimate the $\rho_{D}$ contribution:

$$
\begin{aligned}
& b^{4} \int \rho_{\mathrm{Tot}}^{2}\left\{\sum_{j_{1}+j_{2}=k, j_{2} \geq 2} \frac{\left|\nabla^{j_{1}+1} \Delta \rho_{\mathrm{Tot}}\right|^{2}}{\rho_{\mathrm{Tot}}^{2}\langle Z\rangle^{2 j_{2}}}+\frac{\left|\nabla^{j_{1}} \Delta \rho_{\mathrm{Tot}}\right|^{2}}{\rho_{\mathrm{Tot}}^{2}\langle Z\rangle^{2 j_{2}+2}}\right\} \\
& \lesssim b^{4} \sum_{j_{1}+j_{2}=k, j_{2} \geq 2}\left[\int \frac{\rho_{\mathrm{Tot}}^{2} d Z}{\langle Z\rangle^{2 j_{2}+2\left(j_{1}+3\right)}}+\int \frac{\left|\nabla^{j_{1}+3} \tilde{\rho}\right|^{2}}{\langle Z\rangle^{2 j_{2}}}\right] \\
& \lesssim b^{4}\left(1+\sum_{j_{1}=2}^{k} \int \frac{\left|\nabla \nabla^{j_{1}} \tilde{\rho}\right|^{2}}{\langle Z\rangle^{2\left(k-j_{1}\right)+2}}\right) \leq d_{0}+\delta J_{k_{m}} .
\end{aligned}
$$


In the general case, we replace $\left(\nabla^{i} \rho_{\mathrm{Tot}}\right)^{m_{i}}$ by $\left(\nabla^{i} \hat{\rho}\right)^{m_{i}}$ where $\hat{\rho}$ is either $\rho_{D}$ or $\tilde{\rho}$. In both cases we will use the weaker estimates (4.40).

First, assume that $m_{i}=0$ for $i \geq \frac{4 k_{m}}{9}+1$, then from (4.40):

$$
\begin{aligned}
\left|N_{j_{1}, j_{2}}\right| & \lesssim b^{2}\left|\nabla^{j_{1}+1} \Delta \rho_{\mathrm{Tot}}\right| \frac{1}{\rho_{\text {Tot }}^{j_{2}+1}} \sum_{m_{1}+2 m_{1}+\cdots+j_{2} m_{j_{2}}=j_{2}} \Pi_{i=0}^{j_{2}}\left|\left(\nabla^{i} \hat{\rho}\right)^{m_{i}}\right| \\
& \lesssim b^{2} \frac{\left|\nabla^{j_{1}+1} \Delta \rho_{\mathrm{Tot}}\right|}{\rho_{\mathrm{Tot}}\langle Z\rangle^{j_{2}}}
\end{aligned}
$$

and the conclusion follows as above. Otherwise, there are at most two value $\frac{4 k_{m}}{9} \leq i_{1} \leq i_{2} \leq j_{2}$ with $m_{i_{1}}, m_{i_{2}} \neq 0$ and $m_{i_{1}}+m_{i_{2}} \leq 2$. Hence from (4.40):

$$
\begin{aligned}
\frac{1}{\rho_{\mathrm{Tot}}^{j_{2}+1}} \Pi_{i=0}^{j_{2}}\left|\left(\nabla^{i} \tilde{\rho}\right)^{m_{i}}\right| & \lesssim \frac{1}{\rho_{D}^{j_{2}+1}}\left|\nabla^{i_{1}} \tilde{\rho}\right|^{m_{i_{1}}}\left|\nabla^{i_{2}} \hat{\rho}\right|^{m_{i_{2}}} \Pi_{0 \leq i \leq j_{2}, i \notin\left\{i_{1}, i_{2}\right\}}\left(\frac{\rho_{D}}{\langle Z\rangle^{i}}\right)^{m_{i}} \\
& \lesssim\left(\frac{\left|\nabla^{i_{1}} \hat{\rho}\right|}{\rho_{D}}\right)^{m_{i_{1}}}\left(\frac{\left|\nabla^{i_{2}} \hat{\rho}\right|}{\rho_{D}}\right)^{m_{i_{2}}} \frac{1}{\rho_{D}\langle Z\rangle^{j_{2}-\left(m_{i_{1}} i_{1}+m_{i_{2}} i_{2}\right)}}
\end{aligned}
$$

Assume first $i_{2} \geq \frac{2 k_{m}}{3}+1$, then $m_{i_{1}}=0, m_{i_{2}}=1$ and $j_{1}+3 \leq \frac{4 k_{m}}{9}$ from which:

$$
\begin{aligned}
\int \rho_{\text {Tot }}^{2}\left|N_{j_{1}, j_{2}}\right|^{2} & \lesssim b^{4} \int \rho_{\text {Tot }}^{2}\left|\nabla^{j_{1}+1} \Delta \rho_{\text {Tot }}\right|^{2} \frac{\left|\nabla^{i_{2}} \hat{\rho}\right|^{2}}{\rho_{D}^{2}} \frac{1}{\rho_{D}^{2}\langle Z\rangle^{2\left(j_{2}-i_{2}\right)}} \\
& \lesssim b^{4} \int \frac{\left|\nabla^{i_{2}} \hat{\rho}\right|^{2}}{\langle Z\rangle^{2\left(j_{2}-i_{2}\right)+2\left(j_{1}+3\right)}} \\
& \lesssim b^{4} \int \frac{\left|\nabla^{i_{2}} \hat{\rho}\right|^{2}}{\langle Z\rangle^{2\left(k-i_{2}\right)+6}} \leq d_{0}
\end{aligned}
$$

There remains the case $\frac{4 k_{m}}{9}+1 \leq i_{1} \leq i_{2} \leq \frac{2 k_{m}}{3}$ which imply $j_{1}+3 \leq \frac{2 k_{m}}{3}$, and we distinguish cases:

- case $\left(m_{i_{1}}, m_{i_{2}}\right)=(0,1)$ : if $j_{1}+3 \leq \frac{4 k_{m}}{9}$, we estimate

$$
\begin{aligned}
\int \rho_{\mathrm{Tot}}^{2}\left|N_{j_{1}, j_{2}}\right|^{2} & \lesssim b^{4} \int \rho_{D}^{2}\left|\nabla^{j_{1}+1} \Delta \rho_{\mathrm{Tot}}\right|^{2} \frac{\left|\nabla^{i_{2}} \hat{\rho}\right|^{2}}{\rho_{D}^{2}} \frac{1}{\rho_{D}^{2}\langle Z\rangle^{2\left(j_{2}-i_{2}\right)}} \\
& \lesssim b^{4} \int \frac{\left|\nabla^{i_{2}} \hat{\rho}\right|^{2}}{\langle Z\rangle^{2\left(j_{2}-i_{2}\right)+2\left(j_{1}+3\right)}} \\
& \lesssim b^{4} \int \frac{\left|\nabla^{i_{2}} \hat{\rho}\right|^{2}}{\langle Z\rangle^{2\left(k-i_{2}\right)+6}} \leq d_{0} .
\end{aligned}
$$


Otherwise, $\frac{4 k_{m}}{9}+1 \leq j_{1}+3 \leq \frac{2 k_{m}}{3}$. Hence $\frac{4 k_{m}}{9}+1 \leq j_{1}+3 \leq \frac{2 k_{m}}{3}$, $\frac{4 k_{m}}{9}+1 \leq i_{2} \leq \frac{2 k_{m}}{3}$ and we estimate from (4.40), using $k_{m}$ large:

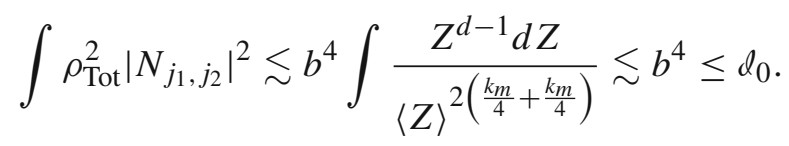

- case $m_{i_{1}}+m_{i_{2}}=2:$ we obtain from (4.40) and $j_{1}+3 \leq \frac{2 k_{m}}{3}$

$$
\int \rho_{\mathrm{Tot}}^{2}\left|N_{j_{1}, j_{2}}\right|^{2} \lesssim b^{4} \int \rho_{D}^{2}\left|\partial^{j_{1}+1} \Delta \rho_{\mathrm{Tot}}\right|^{2}\left(\frac{1}{\langle Z\rangle^{\frac{k_{m}}{4}}}\right)^{4} \lesssim b^{4} \int \frac{d Z}{\langle Z\rangle^{k_{m}}} \leq d_{0}
$$

Step $6 \mathrm{NL}(\tilde{\rho})$ term. We need to estimate

$$
\int \rho_{\mathrm{Tot}}^{2} \nabla \Delta^{K_{m}} \mathrm{NL}(\tilde{\rho}) \cdot \nabla \Psi_{k}
$$

which requires an integration by part in time for the highest order term. We expand using that, according to our assumptions, the nonlinearity is an integer:

$$
\mathrm{NL}(\tilde{\rho})=\left(\rho_{D}+\tilde{\rho}\right)^{p-1}-\rho_{D}^{p-1}-(p-1) \rho_{D}^{p-2} \tilde{\rho}=\sum_{q=2}^{p-1} c_{q} \tilde{\rho}^{q} \rho_{D}^{p-1-q}
$$

and hence by Leibniz:

$$
\begin{aligned}
\Delta^{K_{m}} \mathrm{NL}(\tilde{\rho})= & \sum_{q=2}^{p-1} c_{q} \tilde{\rho}^{q-1}\left(\Delta^{K_{m}} \tilde{\rho}\right) \rho_{D}^{p-1-q} \\
& +\sum_{q=2}^{p-1} \sum_{j_{1}+j_{2}=k \ell_{1}+\cdots+\ell_{q}=j_{1}, \ell_{1} \leq \cdots \ell_{q} \leq k-1} \nabla^{\ell_{1}} \tilde{\rho} \cdots \nabla^{\ell_{q}} \tilde{\rho} \nabla^{j_{2}}\left(\rho_{D}^{p-1-q}\right)
\end{aligned}
$$

Let

$$
N_{\ell_{1}, \ldots, \ell_{q}, j_{1}, q}=\nabla^{\ell_{1}} \tilde{\rho} \cdots \nabla^{\ell_{q}} \tilde{\rho} \nabla^{j_{2}}\left(\rho_{D}^{p-1-q}\right), \quad \ell_{1} \leq \cdots \leq \ell_{q}
$$

case $\ell_{q} \leq k_{m}-2$ : we estimate

$$
\left|\nabla N_{\ell_{1}, \ldots, \ell_{q}, j_{1}, q}\right| \lesssim\left|\nabla^{m_{1}} \tilde{\rho} \cdots \nabla^{m_{q}} \tilde{\rho}\right| \frac{\rho_{D}^{p-1-q}}{\langle Z\rangle^{j_{2}}}, \quad \begin{aligned}
& 0 \leq m_{i} \leq k_{m}-1 \\
& m_{1}+\cdots m_{q}=j_{1}+1 .
\end{aligned}
$$


We may reorder $m_{1} \leq \cdots \leq m_{q}$. If $m_{q} \leq \frac{4 k_{m}}{9}$, then

$$
\left|\nabla N_{\ell_{1}, \ldots, \ell_{q}, j_{1}, q}\right| \lesssim \frac{\tilde{\rho}^{q}}{\langle Z\rangle^{j_{1}+1}} \frac{\rho_{D}^{p-1-q}}{\langle Z\rangle^{j_{2}}} \lesssim \frac{d_{0}}{\langle Z\rangle^{\frac{k_{m}}{2}}}
$$

and hence the contribution of this term

$$
\int \rho_{\text {Tot }}^{2}\left|\nabla N_{\ell_{1}, \ldots, \ell_{q}, j_{1}, q}\right|^{2} \leq d_{0}
$$

If $\frac{4 k_{m}}{9} \leq m_{q} \leq \frac{2 k_{m}}{3}$, then similarly, combining (6.9), (6.1):

$$
\left|\nabla N_{\ell_{1}, \ldots, \ell_{q}, j_{1}, q}\right| \lesssim \frac{1}{\langle Z\rangle^{j_{2}}} \frac{d_{0}}{\langle Z\rangle^{\frac{k_{m}}{4}}}
$$

and the conclusion follows. If $m_{q} \geq \frac{2 k_{m}}{3}$, then $m_{q-1} \leq \frac{4 k_{m}}{9}$ from which:

$$
\left|\nabla N_{\ell_{1}, \ldots, \ell_{q}, j_{1}, q}\right| \lesssim \frac{\rho_{D}^{p-1-q}}{\langle Z\rangle^{j_{2}}} \frac{\rho_{D}^{q-1}}{\langle Z\rangle^{j_{1}+1-\ell_{q}}}\left|\nabla^{\ell_{q}} \tilde{\rho}\right| \lesssim \frac{\rho_{D}^{p-2}\left|\nabla^{\ell_{q}} \tilde{\rho}\right|}{\langle Z\rangle^{k_{m}+1-m_{q}}}
$$

and hence the bound

$$
\begin{aligned}
\int \rho_{\mathrm{Tot}}^{2}\left|\nabla N_{\ell_{1}, \ldots, \ell_{q}, j_{1}, q}\right|^{2} & \lesssim \int \rho_{\mathrm{Tot}}^{2(p-2)+2} \frac{\left|\nabla^{m_{q}} \tilde{\rho}\right|^{2}}{\langle Z\rangle^{2\left(k_{m}-m_{q}\right)+2}} \\
& \lesssim\|\tilde{\rho}, \Psi\|_{k_{m}-1, \sigma\left(k_{m}-1\right)}^{2} \leq d_{0} .
\end{aligned}
$$

case $\ell_{q}=k_{m}-1$ : we compute $\nabla N_{\ell_{1}, \ldots, \ell_{q}, j_{1}, q}$. If the derivative falls on $\ell_{j}$, $j \leq q-1$, we are back to the previous case, and we are therefore left with estimating

$$
\left|\partial^{\ell_{1}} \tilde{\rho} \partial^{\ell_{q-1}} \tilde{\rho} \partial^{k_{m}} \tilde{\rho}\right| \frac{\rho_{D}^{p-1-q}}{\langle Z\rangle^{j_{2}}}, \quad \begin{aligned}
& \ell_{1}+\cdots+\ell_{q-1}+k_{m}=j_{1}+1 \\
& j_{1}+j_{2}=k_{m} .
\end{aligned}
$$

If $j_{1}=k_{m}-1$, then $j_{2}=1, \ell_{1}=\cdots=\ell_{q-1}=0$ and we estimate relying onto the smallness of $\frac{\tilde{\rho}}{\rho_{\mathrm{Tot}}}$ from (7.4) (for $Z \leq Z^{*}$ ) and using (4.40) together with the smallness of $\langle Z\rangle^{-1}$ (for $Z \geq Z^{*}$ ):

$$
\left|\nabla^{\ell_{1}} \tilde{\rho} \cdots \nabla^{\ell_{q-1}} \tilde{\rho} \nabla^{k_{m}} \tilde{\rho}\right| \frac{\rho_{D}^{p-1-q}}{\langle Z\rangle^{j_{2}}} \lesssim\left|\tilde{\rho}^{q-1} \nabla^{k_{m}} \tilde{\rho}\right| \frac{\rho_{D}^{p-1-q}}{\langle Z\rangle} \lesssim \alpha_{0} \rho_{D}^{p-2}\left|\nabla^{k_{m}} \tilde{\rho}\right|
$$


and hence the corresponding contribution $(p \geq 3)$

$$
d_{0} \int \rho_{D}^{2(p-2)}\left|\partial^{k_{m}} \tilde{\rho}\right|^{2} \leq \delta J_{k_{m}} .
$$

Similarly, if $j_{1}=k_{m}$ then $\ell_{1}=\cdots=\ell_{q-2}=j_{2}=0$ and $\ell_{q-1}=1$.

$$
\begin{aligned}
\left|\nabla^{\ell_{1}} \tilde{\rho} \cdots \nabla^{\ell_{q-1}} \tilde{\rho} \nabla^{k_{m}} \tilde{\rho}\right| \frac{\rho_{D}^{p-1-q}}{\langle Z\rangle^{j_{2}}} & \lesssim \tilde{\rho}^{q-2}|\nabla \tilde{\rho}|\left|\nabla^{k_{m}} \tilde{\rho}\right| \rho_{D}^{p-1-q} \\
& \lesssim \ell_{0} \rho_{D}^{p-2}\left|\nabla^{k_{m}} \tilde{\rho}\right|
\end{aligned}
$$

Highest order term We are left with estimating the highest order term:

$$
N_{\ell_{1}, \ldots, \ell_{q}, j_{1}, q}=\tilde{\rho}^{q-1} \rho_{D}^{p-1-q} \Delta^{K_{m}} \tilde{\rho} .
$$

We treat this term by integration by parts in time using (7.8):

$$
\begin{aligned}
- & \int \rho_{\mathrm{Tot}}^{2} \nabla\left[\tilde{\rho}^{q-1} \rho_{D}^{p-1-q} \Delta^{K_{m}} \tilde{\rho}\right] \cdot \nabla \Psi_{k} \\
= & \int \tilde{\rho}^{q-1} \rho_{D}^{p-1-q} \tilde{\rho}_{k} \nabla \cdot\left(\rho_{\mathrm{Tot}}^{2} \nabla \Psi_{k}\right) \\
= & -\int \tilde{\rho}^{q-1} \rho_{D}^{p-1-q} \tilde{\rho}_{k} \rho_{\mathrm{Tot}}\left[\partial_{\tau} \tilde{\rho}_{k}-\left(H_{1}-k\left(H_{2}+\Lambda H_{2}\right)\right) \tilde{\rho}_{k}\right. \\
& +H_{2} \Lambda \tilde{\rho}_{k}+\left(\Delta^{K_{m}} \rho_{\mathrm{Tot}}\right) \Delta \Psi \\
& \left.+k \nabla \rho_{\mathrm{Tot}} \cdot \nabla \Psi_{k}+2 \nabla\left(\Delta^{K_{m}} \rho_{\mathrm{Tot}}\right) \cdot \nabla \Psi-F_{1}\right]
\end{aligned}
$$

and we treat all terms in (7.18). We will systematically use the smallness (4.27). The $\partial_{\tau} \tilde{\rho}_{k}$ term is integrated by parts in time:

$$
\begin{aligned}
& -\int \tilde{\rho}^{q-1} \rho_{D}^{p-1-q} \rho_{\mathrm{Tot}} \tilde{\rho}_{k} \partial_{\tau} \tilde{\rho}_{k}=-\frac{1}{2} \frac{d}{d \tau}\left\{\int \tilde{\rho}^{q-1} \rho_{D}^{p-1-q} \rho_{\mathrm{Tot}} \tilde{\rho}_{k}^{2}\right\} \\
& +\frac{1}{2} \int \tilde{\rho}_{k}^{2} \partial_{\tau}\left(\tilde{\rho}^{q-1} \rho_{D}^{p-1-q} \rho_{\mathrm{Tot}}\right) \\
& =-\frac{1}{2} \frac{d}{d \tau}\left\{\int \tilde{\rho}^{q-1} \rho_{D}^{p-1-q} \rho_{\mathrm{Tot}} \tilde{\rho}_{k}^{2}\right\}+O\left(\delta \int \rho_{D}^{p-1} \tilde{\rho}_{k}^{2}\right)
\end{aligned}
$$

and the boundary term in time is small

$$
\int \tilde{\rho}^{q-1} \rho_{D}^{p-1-q} \rho_{\mathrm{Tot}} \tilde{\rho}_{k}^{2} \lesssim \delta \int \rho_{D}^{p-1} \rho_{k}^{2} .
$$


We then estimate:

$$
\begin{aligned}
& \left|\int \tilde{\rho}^{q-1} \rho_{D}^{p-1-q} \tilde{\rho}_{k} \rho_{\operatorname{Tot}}\left(H_{1}-k\left(H_{2}+\Lambda H_{2}\right)\right) \tilde{\rho}_{k}\right| \\
& \quad \lesssim k \delta \int \rho_{\text {Tot }}^{p-1} \tilde{\rho}_{k}^{2} \lesssim \delta J_{k_{m}} .
\end{aligned}
$$

Using the extra decay in $Z$ and $\|\Delta \Psi\|_{L^{\infty}} \leq \delta \ll 1$ :

$$
\begin{aligned}
& \left|\int \tilde{\rho}^{q-1} \rho_{D}^{p-1-q} \tilde{\rho}_{k} \rho_{\mathrm{Tot}}\left(\Delta^{K_{m}} \rho_{\mathrm{Tot}}\right) \Delta \Psi\right| \lesssim \iota_{0} \\
& \int \frac{d Z}{\langle Z\rangle^{\frac{k_{m}}{2}}}+\int \rho_{\mathrm{Tot}}^{p-1} \tilde{\rho}_{k}^{2}|\Delta \Psi| \leq \iota_{0}+\delta J_{k_{m}} .
\end{aligned}
$$

Similarly, after an integration by parts:

$$
\begin{aligned}
& \left|-\int \tilde{\rho}^{q-1} \rho_{D}^{p-1-q} \tilde{\rho}_{k} \rho_{\mathrm{Tot}} \nabla\left(\Delta^{K_{m}} \rho_{\mathrm{Tot}}\right) \cdot \nabla \Psi\right| \lesssim \iota_{0} \\
& +\left|\int \tilde{\rho}^{q-1} \rho_{D}^{p-1-q} \rho_{\mathrm{Tot}} \nabla\left(\tilde{\rho}_{k}^{2}\right) \cdot \nabla \Psi\right| \leq d_{0}+\delta J_{k_{m}} .
\end{aligned}
$$

Similarly, after an integration by parts using (4.40):

$$
\begin{aligned}
& \left|-\int \tilde{\rho}^{q-1} \rho_{D}^{p-1-q} \tilde{\rho}_{k} \rho_{\operatorname{Tot}} H_{2} \Lambda \tilde{\rho}_{k}\right| \lesssim \iota_{0} \\
& \quad+\left(\left\|\frac{\tilde{\rho}}{\rho_{\mathrm{Tot}}}\right\|_{L^{\infty}}+\left\|\frac{Z|\nabla \tilde{\rho}|}{\rho_{\mathrm{Tot}}}\right\|_{L^{\infty}}\right) J_{k_{m}} \leq \iota_{0}+\delta J_{k_{m}}
\end{aligned}
$$

and similarly

$$
\left|\int \tilde{\rho}^{q-1} \rho_{D}^{p-1-q} \tilde{\rho}_{k} k \nabla \rho_{\mathrm{Tot}} \cdot \nabla \Psi_{k}\right| \lesssim \alpha_{0}+\delta J_{k_{m}} .
$$

Step 7 Conclusion for $k=k_{m}(d, r)$ large enough. We now sum the collection of above bounds and obtain the differential inequality with $k=k_{m}$.

$$
\begin{aligned}
& \frac{1}{2} \frac{d}{d \tau}\left\{J_{k_{m}}(1+O(\delta))\right\} \\
& \leq-k\left[1+O\left(\frac{1}{k}\right)\right] \int\left(H_{2}+\Lambda H_{2}\right) \\
& \quad \times\left[b^{2}\left|\nabla \tilde{\rho}_{k}\right|^{2}+(p-1) \rho_{D}^{p-2} \rho_{\mathrm{Tot}} \tilde{\rho}_{k}^{2}+\rho_{\mathrm{Tot}}^{2}\left|\nabla \Psi_{k}\right|^{2}\right]
\end{aligned}
$$




$$
-k \int(p-1) \rho_{D} \partial_{Z}\left(\rho_{D}^{p-1}\right) \tilde{\rho}_{k} \partial_{Z} \Psi_{k}+\ell .
$$

We recall from (2.21), (2.22):

$$
H_{2}+\Lambda H_{2}=\mu(1-w-\Lambda w) \geq c_{d, p}>0
$$

and we now claim the pointwise coercivity of the coupled quadratic form: $\exists c_{d, p}>0$ such that $\forall Z \geq 0$,

$$
\begin{aligned}
& \left(H_{2}+\Lambda H_{2}\right)\left[(p-1) \rho_{D}^{p-2} \rho_{\mathrm{Tot}} \tilde{\rho}_{k}^{2}+\rho_{\mathrm{Tot}}^{2}\left|\nabla \Psi_{k}\right|^{2}\right] \\
& \quad+(p-1) \rho_{D} \partial_{Z}\left(\rho_{D}^{p-1}\right) \tilde{\rho}_{k} \partial_{Z} \Psi_{k} \\
& \geq c_{d, p}\left[(p-1) \rho_{D}^{p-2} \rho_{\mathrm{Tot}} \tilde{\rho}_{k}^{2}+\rho_{\mathrm{Tot}}^{2}\left|\nabla \Psi_{k}\right|^{2}\right]
\end{aligned}
$$

which, after taking $k>k^{*}(d, p)$ large enough, concludes the proof of (7.6). Proof of (7.20). The coupling term is lower order for $Z$ large:

$$
\begin{aligned}
& \left|(p-1) \rho_{D} \partial_{Z}\left(\rho_{D}^{p-1}\right) \tilde{\rho}_{k} \partial_{Z} \Psi_{k}\right| \lesssim \frac{\rho_{\mathrm{Tot}}^{p-1}}{\langle Z\rangle} \tilde{\rho}_{k} \rho_{\mathrm{Tot}} \partial_{Z} \Psi_{k} \\
& \quad \leq \delta\left[(p-1) \rho_{D}^{p-2} \rho_{\operatorname{Tot}} \tilde{\rho}_{k}^{2}+\rho_{\mathrm{Tot}}^{2}\left|\nabla \Psi_{k}\right|^{2}\right]
\end{aligned}
$$

for $Z>Z(\delta)$ large enough. On a compact set using the smallness (4.27), (7.20) is implied by:

$$
\begin{aligned}
& \left(H_{2}+\Lambda H_{2}\right)\left[(p-1) Q \tilde{\rho}_{k}^{2}+\rho_{P}^{2}\left|\nabla \Psi_{k}\right|^{2}\right]+(p-1) \rho_{P} \partial_{Z} Q \tilde{\rho}_{k} \partial_{Z} \Psi_{k} \\
& \geq c_{d, p}\left[(p-1) Q \tilde{\rho}_{k}^{2}+\rho_{P}^{2}\left|\nabla \Psi_{k}\right|^{2}\right] .
\end{aligned}
$$

We compute the discriminant:

$$
\begin{aligned}
\text { Discr } & =(p-1)^{2} \rho_{P}^{2}\left(\partial_{Z} Q\right)^{2}-4 \mu^{2}(p-1) \rho_{P}^{2} Q\left(H_{2}+\Lambda H_{2}\right)^{2} \\
& =(p-1) \rho_{P}^{2} Q\left[(p-1) \frac{\left(\partial_{Z} Q\right)^{2}}{Q}-4 \mu^{2}(1-w-\Lambda w)^{2}\right] .
\end{aligned}
$$

We compute from (2.10):

$$
\begin{aligned}
(p-1) \frac{\left(\partial_{Z} Q\right)^{2}}{Q} & =(p-1)\left(2 \partial_{Z} \sqrt{Q}\right)^{2} \\
& =(p-1)\left(\frac{1-e}{2} \sqrt{\ell} \partial_{Z}(\sigma Z)\right)^{2}=(1-e)^{2}\left(\partial_{Z}(Z \sigma)\right)^{2}
\end{aligned}
$$




$$
=\frac{4}{r^{2}}\left(\partial_{Z}(Z \sigma)\right)^{2}=4 \mu^{2}(\sigma+\Lambda \sigma)^{2}
$$

and hence from (2.21), (2.22) the lower bound:

$$
\begin{aligned}
-\operatorname{Discr} & =4 \mu^{2}(p-1) \rho_{P}^{2} Q\left[(1-w-\Lambda w)^{2}-(\sigma+\Lambda \sigma)^{2}\right] \\
& \geq c_{d, r}(p-1) \rho_{P}^{2} Q, \quad c_{d, r}>0,
\end{aligned}
$$

which together with (7.19) concludes the proof of (7.20).

\section{Control of low Sobolev norms and proof of Theorem 1.1}

Our aim in this section is to control weighted low Sobolev norms in the interior $r \leq 1\left(Z \leq Z^{*}\right)$. On our way we will conclude the proof of the bootstrap Proposition 4.4. Theorem 1.1 will then follow from a classical topological argument.

\subsection{Exponential decay slightly beyond the light cone}

We use the exponential decay estimate (3.5) for a linear problem to prove exponential decay for the nonlinear evolution in the region slightly past the light cone $Z=Z_{2}$. We recall the notations of Sect. 3, in particular $Z_{a}$ of Lemma 3.7.

Lemma 8.1 (Exponential decay slightly past the light cone) Let

$$
\tilde{Z}_{a}=\frac{Z_{2}+Z_{a}}{2}
$$

Then, there holds the following bound:

$$
\|\nabla \Phi\|_{H^{2 k_{0}}\left(Z \leq \tilde{Z}_{a}\right)}+\|\rho\|_{H^{2 k_{0}}\left(Z \leq \tilde{Z}_{a}\right)} \lesssim e^{-\frac{\delta_{g}}{2} \tau} .
$$

Proof The proof relies on the spectral theory beyond the light cone $Z=Z_{2}$ and an elementary finite speed propagation like argument in renormalized variables, related to [48].

Step 1 Semigroup decay in $X$ variables. Recall the definition (4.12) of $X=$ $(\Phi, T)$

$$
\mid \begin{aligned}
& \Phi=\rho_{P} \Psi \\
& T=\partial_{\tau} \Phi+a H_{2} \Lambda \Phi \\
& \quad=-(p-1) Q \rho-H_{2} \Lambda \Phi+\left(H_{1}-e\right) \Phi+G_{\Phi}+a H_{2} \Lambda \Phi
\end{aligned}
$$


with $G_{\Phi}$ given by (3.11), the scalar product (3.44) and the definitions (4.14), (4.15):

$$
\mid \begin{aligned}
& \Lambda_{0}=\{\lambda \in \mathbb{C}, \quad \Re(\lambda) \geq 0\} \cap\{\lambda \text { is an eigenvalue of } m\}=\left(\lambda_{i}\right)_{1 \leq i \leq N}, \\
& V=\cup_{1 \leq i \leq N} \operatorname{ker}\left(m-\lambda_{i} I\right)^{k_{\lambda_{i}}}
\end{aligned}
$$

the projection $\mathbb{P}$ associated with $V$, the decay estimate (3.5) on the range of $(I-\mathbb{P})$ and the results of Lemma 3.5. Relative to the $X$ variables our equations take the form

$$
\partial_{\tau} X=m X+G
$$

which are considered on the time interval $\tau \geq \tau_{0} \gg 1$ and the space interval $Z \in\left[0, Z_{a}\right]$ (no boundary conditions at $Z_{a}$ ). We consider evolution in the Hilbert space $\mathbb{H}_{2 k_{0}}$ with initial data such that

$$
\left\|(I-\mathbb{P}) X\left(\tau_{0}\right)\right\|_{\mathbb{H}_{2 k_{0}}} \leq e^{-\frac{\delta g}{2} \tau_{0}}, \quad\left\|\mathbb{P} X\left(\tau_{0}\right)\right\|_{\mathbb{H}_{2 k_{0}}} \leq e^{-\frac{3 \delta_{g}}{5} \tau_{0}} .
$$

According to the bootstrap assumption (4.45)

$$
\|\mathbb{P} X(\tau)\|_{\mathbb{H}_{2 k_{0}}} \leq e^{-\frac{\delta g}{2} \tau}, \quad \forall \tau \in\left[\tau_{0}, \tau^{*}\right]
$$

Lemma 3.5 shows that as long as

$$
\|G\|_{\mathbb{H}_{2 k_{0}}} \leq e^{-\frac{2 \delta_{g}}{3} \tau}, \quad \tau \geq \tau_{0}
$$

there exists $\Gamma$, which can be made as large as we want with a choice of $\tau_{0}$, such that

$$
\|\mathbb{P} X(\tau)\|_{\mathbb{H}_{2 k_{0}}} \lesssim e^{-\frac{\delta g}{2} \tau}, \quad \tau_{0} \leq \tau \leq \tau_{0}+\Gamma
$$

This will allow us to show eventually that if we can verify (8.5), the bootstrap time $\tau^{*} \geq \tau_{0}+\Gamma$.

Moreover, as long as (8.5) holds, the decay estimate (3.5) implies that

$$
\begin{aligned}
\|(I-\mathbb{P}) X(\tau)\|_{\mathbb{H}_{2 k_{0}} \lesssim} & e^{-\frac{\delta g}{2}\left(\tau-\tau_{0}\right)}\left\|X\left(\tau_{0}\right)\right\|_{\mathbb{H}_{2 k_{0}}} \\
& +\int_{\tau_{0}}^{\tau} e^{-\frac{\delta g}{2}(\tau-\sigma)}\|G(\sigma)\|_{\mathbb{H}_{2 k_{0}}} d \sigma \\
\lesssim & e^{\frac{-\delta g}{2} \tau}\left[e^{\frac{\delta g}{2} \tau_{0}}\left\|X\left(\tau_{0}\right)\right\|_{\mathbb{H}_{2 k_{0}}}+\int_{\tau_{0}}^{+\infty} e^{-\frac{\delta g}{6} \tau} d \tau\right]
\end{aligned}
$$




$$
\leq e^{-\frac{\delta g}{2} \tau}
$$

As a result,

$$
\|X(\tau)\|_{\mathbb{H}_{2 k_{0}}} \lesssim e^{-\frac{\delta_{g}}{2} \tau}, \quad \tau_{0} \leq \tau \leq \tau^{*} .
$$

Below we will verify (8.5) $\forall \tau \in\left[\tau_{0}, \tau^{*}\right]$ under the assumption (8.7), closing both. Once again, this will allow us to show eventually that the length of the bootstrap interval $\tau^{*}-\tau_{0} \geq \Gamma$ is sufficiently large.

Recall from (3.13), (3.14), (3.44):

$$
\|G\|_{\mathbb{H}_{2 k_{0}}}^{2} \lesssim \int_{Z \leq Z_{a}}\left|\nabla \Delta^{k_{0}} G_{T}\right|^{2} g Z^{d-1} d Z+\int_{Z \leq Z_{a}} G_{T}^{2} Z^{d-1} d Z
$$

with

$$
\mid \begin{aligned}
& G_{T}=\partial_{\tau} G_{\Phi}-\left(H_{1}+H_{2} \frac{\Lambda Q}{Q}\right) G_{\Phi}+H_{2} \Lambda G_{\Phi}-(p-1) Q G_{\rho}, \\
& G_{\rho}=-\rho \Delta \Psi-2 \nabla \rho \cdot \nabla \Psi \\
& G_{\Phi}=-\rho_{P}\left(|\nabla \Psi|^{2}+\mathrm{NL}(\rho)\right)+\frac{b^{2} \rho_{P}}{\rho_{\mathrm{Tot}}} \Delta \rho_{\mathrm{Tot}} .
\end{aligned}
$$

Step 2 Semigroup decay for $(\rho, \Psi)$. We now translate the $X$ bound to the bounds for $\rho$ and $\Psi$ and then verify (8.5). We recall (8.2) and obtain for any $\hat{Z}>Z_{2}$

$$
\begin{aligned}
& \|T\|_{H^{2 k_{0}(Z \leq \hat{Z})}}+\|\Phi\|_{H^{2 k_{0}+1}(Z \leq \hat{Z})} \\
& \quad \lesssim\|\rho\|_{H^{2 k_{0}(Z \leq \hat{Z})}}+\|\Psi\|_{H^{2 k_{0}+1}(Z \leq \hat{Z})}+\left\|G_{\Phi}\right\|_{H^{2 k_{0}}(Z \leq \hat{Z})} \\
& \quad \lesssim\|T\|_{H^{2 k_{0}(Z \leq \hat{Z})}}+\|\Phi\|_{H^{2 k_{0}+1}(Z \leq \hat{Z})}+\left\|G_{\Phi}\right\|_{H^{2 k_{0}}(Z \leq \hat{Z})}
\end{aligned}
$$

and claim:

$$
\left\|G_{\Phi}\right\|_{H^{2 k_{0}(Z \leq \hat{Z})}} \lesssim\|\nabla \Psi\|_{H^{2 k_{0}(Z \leq \hat{Z})}}^{2}+\|\rho\|_{H^{2 k_{0}(Z \leq \hat{Z})}}^{2}+e^{-\delta_{g} \tau} .
$$

Indeed, since $H^{2 k_{0}}(Z \leq \hat{Z})$ is an algebra for $k_{0}$ large enough:

$$
\left\|\rho_{P}\left(|\nabla \Psi|^{2}+\mathrm{NL}(\rho)\right)\right\|_{H^{2 k_{0}(Z \leq \hat{Z})}} \lesssim\|\nabla \Psi\|_{H^{2 k_{0}(Z \leq \hat{Z})}}^{2}+\|\rho\|_{H^{2 k_{0}}(Z \leq \hat{Z})}^{2} .
$$

The remaining quantum pressure term is treated using the pointwise bound (4.40) for small Sobolev norms and the smallness of $b$ which imply:

$$
\left\|\frac{b^{2} \rho_{P} \Delta \rho_{\mathrm{Tot}}}{\rho_{\mathrm{Tot}}}\right\|_{H^{2 k_{0}(Z \leq \hat{Z})}} \lesssim C_{K} b^{2} \leq e^{-\delta_{g} \tau}
$$


provided $\delta_{g}>0$ has been chosen small enough, and (8.10) is proved. Choosing $\hat{Z}>Z_{2}$, this implies from (8.2) and the initial bound (4.19):

$$
\begin{aligned}
\left\|X\left(\tau_{0}\right)\right\|_{\mathbb{H}^{2 k_{0}}} & \lesssim\left\|\Psi\left(\tau_{0}\right)\right\|_{H^{2 k_{0}+1}(Z \leq \hat{Z})}+\left\|\rho\left(\tau_{0}\right)\right\|_{H^{2 k_{0}}(Z \leq \hat{Z})}+e^{-\delta_{g} \tau_{0}} \\
& \lesssim e^{-\frac{\delta_{g} \tau_{0}}{2}}
\end{aligned}
$$

This verifies (8.3). On the other hand, choosing $\hat{Z}=\tilde{Z}_{a}$ with

$$
\tilde{Z}_{a}=\frac{Z_{2}+Z_{a}}{2},
$$

we also obtain from (8.8)

$$
\|\Psi(\tau)\|_{H^{2 k_{0}+1}\left(Z \leq \tilde{Z}_{a}\right)}+\|\rho(\tau)\|_{H^{2 k_{0}}\left(Z \leq \tilde{Z}_{a}\right)} \lesssim\|X(\tau)\|_{\mathbb{H}^{2 k_{0}}}+e^{-\delta_{g} \tau} \lesssim e^{-\frac{\delta_{g} \tau}{2}} .
$$

The estimate (8.1) follows.

Step 3 Estimate for $G$. Proof of (8.5). We recall (8.9). On a fixed compact domain $Z \leq Z_{0}$ with $Z_{0}>Z_{2}$, we can interpolate the bootstrap bound (4.39) with the global large Sobolev bound (4.38) and obtain for $k_{m}$ large enough and $b_{0}<b_{0}\left(k_{m}\right)$ small enough:

$$
\|\rho\|_{H^{2 k_{0}+10}\left(Z \leq Z_{0}\right)}+\|\Psi\|_{H^{2 k_{0}+10}\left(Z \leq Z_{0}\right)} \leq C_{K} e^{-\left[\frac{3}{8}-\frac{1}{100}\right] \delta_{g} \tau} \leq e^{-\left[\frac{3}{8}-\frac{1}{50}\right] \delta_{g} \tau}
$$

and since $H^{2 k_{0}}$ is an algebra and all terms are either quadratic or with a $b$ term, (8.13) implies

$$
\begin{aligned}
& \left\|G_{T}\right\|_{H^{2 k_{0}+5}\left(Z \leq Z_{0}\right)}+\left\|G_{\rho}\right\|_{H^{2 k_{0}+5}\left(Z \leq Z_{0}\right)}+\left\|G_{\Phi}\right\|_{H^{2 k_{0}+5}\left(Z \leq Z_{0}\right)} \\
& \leq e^{-\left(\frac{3}{4}-\frac{1}{20}\right) \delta_{g} \tau} \leq e^{-\frac{2 \delta_{g}}{3} \tau}
\end{aligned}
$$

which in particular using (8.9) implies (8.5).

\subsection{Weighted decay for $m \leq 2 k_{0}$ derivatives}

We recall the notation (3.8). We now transform the exponential decay (8.1) from just past the light cone into weighted decay estimate. It is essential for this argument that the decay (8.1) has been shown in the region strictly including the light cone $Z=Z_{2}$. The estimates in the lemma below close the remaining bootstrap bound (4.39). 
Lemma 8.2 (Weighted Sobolev bound for $m \leq 2 k_{0}$ ) Let $m \leq 2 k_{0}$ and $v_{0}=$ $\frac{\delta_{g}}{2 \mu}-\frac{2(r-1)}{p-1}$, recall

$$
\chi_{v_{0}, m}=\frac{1}{\langle Z\rangle^{d-2(r-1)+2\left(v_{0}-m\right)}} \zeta\left(\frac{Z}{Z^{*}}\right), \quad \zeta(Z)=\mid \begin{array}{lll}
1 & \text { for } & Z \leq 2 \\
0 & \text { for } & Z \geq 3
\end{array}
$$

then

$$
\sum_{m=0}^{2 k_{0}} \sum_{i=1}^{d} \int(p-1) Q\left(\partial_{i}^{m} \rho\right)^{2} \chi_{v_{0}, m}+\left|\nabla \partial_{i}^{m} \Phi\right|^{2} \chi_{v_{0}, m} \leq C e^{-\frac{4 \delta_{g}}{5} \tau}
$$

Proof of Lemma 8.2 The proof relies on a sharp energy estimate with time dependent localization of $(\rho, \Phi)$. This is a renormalized version of the finite speed of propagation.

Step $1 \dot{H}^{m}$ localized energy identity. Pick a smooth well localized radially symmetric function $\chi(\tau, Z)$ and a coordinate $1 \leq i \leq d$ and note for $m$ integer

$$
\rho_{m}=\partial_{i}^{m} \rho, \quad \Phi_{m}=\partial_{i}^{m} \Phi,
$$

where we omit the $i$ dependence to simplify notations. We recall the Emden transform formulas (2.24):

$$
\mid \begin{aligned}
& H_{2}=\mu(1-w) \\
& H_{1}=\frac{\mu \ell}{2}(1-w)\left[1+\frac{\Lambda \sigma}{\sigma}\right] \\
& H_{3}=\frac{\Delta \rho_{P}}{\rho_{P}}
\end{aligned}
$$

which yield the bounds using (2.19), (2.20):

$$
\mid \begin{aligned}
& H_{2}=\mu+O\left(\frac{1}{\langle Z\rangle^{r}}\right), \quad H_{1}=-\frac{2 \mu(r-1)}{p-1}+O\left(\frac{1}{\langle Z\rangle^{r}}\right), \\
& \left|\langle Z\rangle^{j} \partial_{Z}^{j} H_{1}\right|+||\langle Z\rangle^{j} \partial_{Z}^{j} H_{2} \mid \lesssim \frac{1}{\langle Z\rangle^{r}}, \quad j \geq 1, \\
& \left|\langle Z\rangle^{j} \partial_{Z}^{j} H_{3}\right| \lesssim \frac{1}{\langle Z\rangle^{2}}, \\
& \frac{1}{\langle Z\rangle^{2(r-1)}}\left[1+O\left(\frac{1}{\langle Z\rangle^{r}}\right)\right] \lesssim_{j}\left|\langle Z\rangle^{j} \partial_{Z}^{j} Q\right| \lesssim_{j} \frac{1}{\langle Z\rangle^{2(r-1)}}
\end{aligned}
$$


and the commutator bounds:

$$
\mid \begin{aligned}
& \left|\left[\partial_{i}^{m}, H_{1}\right] \rho\right| \lesssim \sum_{j=0}^{m-1} \frac{\left|\partial_{Z}^{j} \rho\right|}{\langle Z\rangle^{r+m-j}}, \\
& \left|\nabla\left(\left[\partial_{i}^{m}, H_{1}\right] \rho\right)\right| \lesssim \sum_{j=0}^{m} \frac{\left|\partial_{Z}^{j} \rho\right|}{\langle Z\rangle^{m-j+r+1}}, \\
& \left|\left[\partial_{i}^{m}, Q\right] \rho\right| \lesssim Q \sum_{j=0}^{m-1} \frac{\left|\partial_{Z}^{j} \rho\right|}{\langle Z\rangle^{m-j}}, \\
& \left|\left[\partial_{i}^{m}, H_{2}\right] \Lambda \rho\right| \lesssim \sum_{j=1}^{m} \frac{\left|\partial_{Z}^{j} \rho\right|}{\langle Z\rangle^{r+m-j}}, \\
& \left|\nabla\left(\left[\partial_{i}^{m}, H_{2}\right] \Lambda \Phi\right)\right| \lesssim \sum_{j=1}^{m+1} \frac{\left|\partial_{Z}^{j} \Phi\right|}{\langle Z\rangle^{r+1+m-j}}
\end{aligned}
$$

Commuting (3.9) with $\partial_{i}^{m}$ :

$\mid \begin{aligned} & \partial_{\tau} \rho_{m}=H_{1} \rho_{m}-H_{2}(m+\Lambda) \rho_{m}-\Delta \Phi_{m}+\partial_{i}^{m} G_{\rho}+E_{m, \rho}, \\ & \partial_{\tau} \Phi_{m}=-(p-1) Q \rho_{m}-H_{2}(m+\Lambda) \Phi_{m}+\left(H_{1}-e\right) \Phi_{m}+\partial_{i}^{m} G_{\Phi}+E_{m, \Phi}\end{aligned}$ with the bounds

$$
\mid \begin{aligned}
& \left|E_{m, \rho}\right| \lesssim \sum_{j=0}^{m} \frac{\left|\partial_{Z}^{j} \rho\right|}{\langle Z\rangle^{r-1+m-j}}+\sum_{j=0}^{m} \frac{\left|\partial_{Z}^{j} \Phi\right|}{\langle Z\rangle^{m-j+2}}, \\
& \left|\nabla E_{m, \Phi}\right| \lesssim Q \sum_{j=0}^{m} \frac{\left|\partial_{Z}^{j} \rho\right|}{\langle Z\rangle^{m+1-j}}+\sum_{j=0}^{m+1} \frac{\left|\partial_{Z}^{j} \Phi\right|}{\langle Z\rangle^{r+m-j}} .
\end{aligned}
$$

Let $\chi$ be an arbitrary smooth function. We derive the corresponding energy identity:

$$
\begin{aligned}
\frac{1}{2} & \frac{d}{d \tau}\left\{\int(p-1) Q \rho_{m}^{2} \chi+\left|\nabla \Phi_{m}\right|^{2} \chi\right\} \\
= & \frac{1}{2} \int \partial_{\tau} \chi\left[(p-1) Q \rho_{m}^{2}+\left|\nabla \Phi_{m}\right|^{2}\right] \\
& +\int(p-1) Q \rho_{m} \chi\left[H_{1} \rho_{m}-H_{2}(m+\Lambda) \rho_{m}\right. \\
& \left.-\Delta \Phi_{m}+\partial_{i}^{m} G_{\rho}+E_{m, \rho}\right] \\
& +\int \chi \nabla \Phi_{m} \cdot \nabla\left[-(p-1) Q \rho_{m}-H_{2}\left(m+\Lambda \Phi_{m}\right)\right. \\
& \left.+\left(H_{1}-\mu(r-2)\right) \Phi_{m}+\partial_{m}^{i} G_{\Phi}+E_{m, \Phi}\right] \\
= & \frac{1}{2} \int \partial_{\tau} \chi\left[(p-1) Q \rho_{m}^{2}+\left|\nabla \Phi_{m}\right|^{2}\right] \\
& +\int(p-1) Q \rho_{m} \chi\left[H_{1} \rho_{m}-H_{2}(m+\Lambda) \rho_{m}\right. \\
& \left.+\partial_{i}^{m} G_{\rho}+E_{m, \rho}\right]+\int(p-1) Q \rho_{m} \nabla \chi \cdot \nabla \Phi_{m}
\end{aligned}
$$




$$
\begin{aligned}
& +\int \chi \nabla \Phi_{m} \cdot \nabla\left[-H_{2}(m+\Lambda) \Phi_{m}\right. \\
& \left.+\left(H_{1}-\mu(r-2)\right) \Phi_{m}+\partial_{i}^{m} G_{\Phi}+E_{m, \Phi}\right] .
\end{aligned}
$$

In what follows we will use $\omega>0$ as a small universal constant to denote the power of tails of the error terms. In most cases, the power is in fact $r>2$ which we do not need.

$\rho_{m}$ terms. From the asymptotic behavior of $Q$ (2.20) and (8.17):

$$
\begin{aligned}
& -\int(p-1) Q \rho_{m} \chi H_{2} \Lambda \rho_{m} \\
& =\frac{p-1}{2} \int \rho_{m}^{2} \chi Q H_{2}\left[d+\frac{\Lambda Q}{Q}+\frac{\Lambda H_{2}}{H_{2}}+\frac{\Lambda \chi}{\chi}\right] \\
& =\int \rho_{m}^{2}(p-1) \chi Q \mu\left[\frac{d}{2}-(r-1)+O\left(\frac{1}{\langle Z\rangle^{\omega}}\right)\right] \\
& \quad+\frac{1}{2} \int(p-1) Q H_{2} \Lambda \chi \rho_{m}^{2}
\end{aligned}
$$

$\Phi_{m}$ terms. We first estimate recalling (8.17):

$$
\begin{aligned}
\int & \chi \nabla \Phi_{m} \cdot \nabla\left[\left(-m H_{2}+H_{1}-\mu(r-2)\right) \Phi_{m}\right] \\
= & \int\left(-m H_{2}+H_{1}-\mu(r-2)\right) \chi\left|\nabla \Phi_{m}\right|^{2} \\
& +O\left(\int \frac{\chi}{\langle Z\rangle^{r}}\left|\nabla \Phi_{m}\right|\left|\Phi_{m}\right|\right) \\
= & -\left[\mu(m+r-2)+\frac{2 \mu(r-1)}{p-1}\right] \int \chi\left|\nabla \Phi_{m}\right|^{2} \\
& +O\left(\int \frac{\chi}{\langle Z\rangle^{\omega}}\left[\left|\nabla \Phi_{m}\right|^{2}+\frac{\Phi_{m}^{2}}{\langle Z\rangle^{2}}\right]\right) .
\end{aligned}
$$

From Pohozhaev identity (5.27) with $F=\chi H_{2}\left(Z_{1}, \ldots, Z_{d}\right)$ :

$$
\begin{aligned}
& -\int \chi \nabla \Phi_{m} \cdot \nabla\left(H_{2} \Lambda \Phi_{m}\right)=\int H_{2} \Lambda \Phi_{m}\left[\chi \Delta \Phi_{m}+\nabla \chi \cdot \nabla \Phi_{m}\right] \\
& =-\sum_{i, j=1}^{d} \int \partial_{i} F_{j} \partial_{i} \Phi_{m} \partial_{j} \Phi_{m}+\frac{1}{2} \int\left|\nabla \Phi_{m}\right|^{2} \nabla \cdot F \\
& \quad+\int H_{2} \Lambda \Phi_{m} \nabla \chi \cdot \nabla \Phi_{m}
\end{aligned}
$$




$$
\begin{aligned}
= & \sum_{i, j=1}^{d} \partial_{i} \Phi_{m} \partial_{j} \Phi_{m}\left[-\partial_{i}\left(\chi H_{2} Z_{j}\right)+H_{2} Z_{j} \partial_{i} \chi\right] \\
& +\frac{1}{2} \int\left|\nabla \Phi_{m}\right|^{2} \chi H_{2}\left[d+\frac{\Lambda \chi}{\chi}+\frac{\Lambda H_{2}}{H_{2}}\right] \\
= & \frac{\mu(d-2)}{2} \int \chi\left|\nabla \Phi_{m}\right|^{2}+\frac{1}{2} \int H_{2} \Lambda \chi\left|\nabla \Phi_{m}\right|^{2}+O\left(\int \frac{\chi}{\langle Z\rangle^{\omega}}\left|\nabla \Phi_{m}\right|^{2}\right) .
\end{aligned}
$$

The collection of above bounds yields for some universal constant $\omega>0$ the weighted energy identity:

$$
\begin{aligned}
\frac{1}{2} \frac{d}{d \tau}\left\{\int(p-1) Q \rho_{m}^{2} \chi+\left|\nabla \Phi_{m}\right|^{2} \chi\right\} \\
=-\int \chi\left[(p-1) Q \rho_{m}^{2}+\left|\nabla \Phi_{m}\right|^{2}\right] \\
\times\left[\mu\left(m-\frac{d}{2}+r-1\right)+\frac{2 \mu(r-1)}{p-1}+O\left(\frac{1}{\langle Z\rangle^{\omega}}\right)\right] \\
+\frac{1}{2} \int(p-1) Q \rho_{m}^{2}\left[\partial_{\tau} \chi+H_{2} \Lambda \chi\right] \\
+\frac{1}{2} \int\left|\nabla \Phi_{m}\right|^{2}\left[\partial_{\tau} \chi+H_{2} \Lambda \chi\right]+\int(p-1) Q \rho_{m} \nabla \chi \cdot \nabla \Phi_{m} \\
+O\left(\int \chi \left[\sum_{j=0}^{m+1} \frac{\left|\partial_{Z}^{j} \Phi\right|^{2}}{\left.\left.\langle Z\rangle^{2(m+1-j)+\omega}+\sum_{j=0}^{m} \frac{Q\left|\partial_{Z}^{j} \rho\right|^{2}}{\langle Z\rangle^{2(m-j)+\omega}}\right]\right)}\right.\right. \\
+O\left(\int \chi\left|\nabla \Phi_{m}\right|\left|\nabla \partial^{m} G_{\Phi}\right|+\int \chi Q\left|\rho_{m}\right|\left|\partial^{m} G_{\rho}\right|\right) \cdot
\end{aligned}
$$

Step 2 Nonlinear and source terms. We claim the bound for $\chi=\chi_{\nu_{0}, m}$ :

$$
\begin{gathered}
\sum_{m=0}^{2 k_{0}} \sum_{i=1}^{d} \int \chi_{\nu_{0}, m}\left|\nabla \partial^{m} G_{\Phi}\right|^{2}+\int(p-1) Q \chi_{\nu_{0}, m}\left|\partial^{m} G_{\rho}\right|^{2} \\
\quad \lesssim\left(\sum_{m=0}^{2 k_{0}} \sum_{i=1}^{d} \int Q \rho_{m}^{2} \chi_{\nu_{0}+1, m}+\left|\nabla \Phi_{m}\right|^{2} \chi_{\nu_{0}+1, m}\right)+b^{2}
\end{gathered}
$$

$G_{\rho}$ term. Recall (3.11)

$$
G_{\rho}=-\rho \Delta \Psi-2 \nabla \rho \cdot \nabla \Psi
$$


then by Leibniz:

$$
\left|\partial^{m} G_{\rho}\right|^{2} \lesssim \sum_{j_{1}+j_{2}=m+2, j_{2} \geq 1}\left|\partial^{j_{1}} \rho\right|^{2}\left|\partial^{j_{2}} \Psi\right|^{2}
$$

We recall the pointwise bounds (4.40) for $Z \leq 3 Z^{*}$,

$$
\left|\partial^{j_{1}} \rho\right| \leq \frac{C_{K}}{\langle Z\rangle^{j_{1}+\frac{2(r-1)}{p-1}}}, \quad\left|\partial^{j_{2}} \Psi\right| \leq \frac{C_{K}}{\langle Z\rangle^{j_{2}+r-2}} .
$$

This yields, recalling (8.33), for $j_{1} \leq 2 k_{0}$ :

$$
\begin{aligned}
& \int \chi_{\nu_{0}, m} Q\left|\partial^{j_{1}} \rho\right|^{2}\left|\partial^{j_{2}} \Psi\right|^{2} \lesssim \int Q \zeta\left(\frac{Z}{Z^{*}}\right) \frac{\left|\partial^{j_{1}} \rho\right|^{2}}{Z^{2\left(j_{2}-m\right)+d-2(r-1)+2(r-2)+2 \nu_{0}}} \\
& \lesssim \int \zeta\left(\frac{Z}{Z^{*}}\right) Q \frac{\left|\partial^{j_{1}} \rho\right|^{2}}{\langle Z\rangle^{d-2(r-1)+2\left(\nu_{0}-j_{1}\right)+2}} \lesssim \sum_{j=0}^{j_{1}} \int \chi_{\nu_{0}+1, j_{1}} Q\left|\partial_{Z}^{j} \rho\right|^{2} \\
& \lesssim \sum_{m=0}^{2 k_{0}} \sum_{i=1}^{d} \int Q \rho_{m}^{2} \chi_{\nu_{0}+1, m}+\left|\nabla \Phi_{m}\right|^{2} \chi_{\nu_{0}+1, m} .
\end{aligned}
$$

For $j_{1}=m+1, j_{2}=1$, we use the other variable:

$$
\begin{aligned}
\int & \chi_{v_{0}, m} Q\left|\partial^{j_{1}} \rho\right|^{2}\left|\partial^{j_{2}} \Psi\right|^{2} \lesssim \int Q \zeta\left(\frac{Z}{Z^{*}}\right) \frac{\left|\partial^{j_{2}} \Psi\right|^{2}}{Z^{2\left(j_{1}-m\right)+d-2(r-1)+\frac{4(r-1)}{p-1}+2 v_{0}}} \\
& \lesssim \int \zeta\left(\frac{Z}{Z^{*}}\right) \frac{\rho_{P}^{2}\left|\partial^{j_{2}} \Psi\right|^{2}}{\langle Z\rangle^{d-2(r-1)+2\left(\nu_{0}-j_{2}\right)+2}} \\
& \lesssim \sum_{j=0}^{j_{2}} \int \zeta\left(\frac{Z}{Z^{*}}\right) \frac{\left|\partial_{Z}^{j} \Phi\right|^{2}}{\langle Z\rangle^{d-2(r-1)+2\left(\nu_{0}-j\right)+2}} \\
& \lesssim \sum_{j=0}^{j_{2}} \int \chi_{v_{0}+1, j}\left|\partial_{Z}^{j} \Phi\right|^{2} \lesssim \sum_{m=0}^{2 k_{0}} \sum_{i=1}^{d} \int Q \rho_{m}^{2} \chi_{v_{0}+1, m}+\left|\nabla \Phi_{m}\right|^{2} \chi_{v_{0}+1, m}
\end{aligned}
$$

and (8.20) follows for $G_{\rho}$ by summation on $0 \leq m \leq 2 k_{0}$.

$G_{\Phi}$ term. Recall (3.11)

$$
G_{\Phi}=-\rho_{P}\left(|\nabla \Psi|^{2}+\mathrm{NL}(\rho)\right)+\frac{b^{2} \rho_{P}}{\rho_{\mathrm{Tot}}} \Delta \rho_{\mathrm{Tot}} .
$$


We estimate using the pointwise bounds (4.40) for $j_{3} \leq 2 k_{0}$ :

$$
\begin{aligned}
& \left|\nabla \partial^{m}\left(\rho_{P}|\nabla \Psi|^{2}\right)\right| \lesssim \sum_{j_{1}+j_{2}+j_{3}=m+1, j_{2} \leq j_{3}} \frac{\rho_{P}}{\langle Z\rangle^{j_{1}}}\left|\partial^{j_{2}+1} \Psi \partial^{j_{3}+1} \Psi\right| \\
& \quad \lesssim \sum_{j_{1}+j_{2}+j_{3}=m+1, j_{2} \leq j_{3}} \frac{1}{\langle Z\rangle^{\frac{2(r-1)}{p-1}+j_{1}+r-2+j_{2}+1}}\left|\partial^{j_{3}+1} \Psi\right| \\
& \lesssim \sum_{j_{3}=0}^{2 k_{0}} \frac{\left|\partial^{j_{3}+1} \Phi\right|}{\langle Z\rangle^{r+m-j_{3}}}
\end{aligned}
$$

and since $r>1$ :

$$
\sum_{j_{3}=0}^{2 k_{0}} \int \chi_{v_{0}, m} \frac{\left|\partial^{j_{3}+1} \Phi\right|^{2}}{\langle Z\rangle^{2\left(r+m-j_{3}\right)}} \lesssim \sum_{j_{3}=0}^{2 k_{0}} \int \chi_{\nu_{0}+1, j_{3}}\left|\nabla \Phi_{j_{3}}\right|^{2}
$$

For $j_{3}=2 k_{0}+1$, we use the other variable and the conclusion follows similarly. The quantum pressure term is estimated using the pointwise bounds (4.40):

$$
\begin{aligned}
& \int \chi_{\nu_{0}, m}\left|\nabla \partial^{m}\left(\frac{b^{2} \rho_{P}}{\rho_{\mathrm{Tot}}} \Delta \rho_{\mathrm{Tot}}\right)\right|^{2} \lesssim C_{K} b^{4} \int_{Z \leq 3 Z^{*}} \frac{\chi_{\nu_{0}, m}}{\langle Z\rangle^{\frac{4(r-1)}{p-1}+2(m+3)}} \\
& \lesssim C_{K} b^{4} \int_{Z \leq 3 Z^{*}} \frac{Z^{d-1} d Z}{\langle Z\rangle^{d-2(r-1)+2\left(v_{0}+\frac{2(r-1)}{p-1}-m\right)+2(m+3)}} \leq b^{2}
\end{aligned}
$$

Step 2 Initialization and lower bound on the bootstrap time $\tau^{*}$.

Fix a large enough $Z_{0}$ and pick a small enough universal constant $\omega_{0}$ such that

$$
\forall Z \geq 0, \quad-\omega_{0}+H_{2} \geq \frac{\omega_{0}}{2}>0
$$

and let $\Gamma=\Gamma\left(Z_{0}\right)$ such that

$$
\frac{Z_{0}}{2 \hat{Z}_{a}} e^{-\omega_{0} \Gamma}=1
$$

We claim that provided $\tau_{0}$ has been chosen sufficiently large, the bootstrap time $\tau^{*}$ of Proposition 4.4 satisfies the lower bound

$$
\tau^{*} \geq \tau_{0}+\Gamma
$$


Indeed, in view of Sects. 5, 6, 7 there remains to control the bound (4.39) on $\left[\tau_{0}, \tau_{0}+\Gamma\right]$. By (8.6) (8.7), the desired bounds already hold for $Z \leq \tilde{Z}_{a}$ on $\left[\tau_{0}, \tau_{0}+\Gamma\right]$.

We now run the energy estimate (8.19) with $\chi=\chi_{\nu_{0}, m}$ and obtain from (8.19), (8.20) the rough bound on $\left[\tau_{0}, \tau^{*}\right]$ :

$$
\begin{aligned}
& \frac{d}{d \tau}\left\{\int(p-1) Q \rho_{m}^{2} \chi_{\nu_{0}, m}+\left|\nabla \Phi_{m}\right|^{2} \chi_{\nu_{0}, m}\right\} \\
& \quad \leq C \int(p-1) Q \rho_{m}^{2} \chi_{\nu_{0}, m}+\left|\nabla \Phi_{m}\right|^{2} \chi_{\nu_{0}, m}+b^{2} .
\end{aligned}
$$

which yields using (4.19):

$$
\begin{aligned}
& \int(p-1) Q \rho_{m}^{2} \chi_{v_{0}, m}+\left|\nabla \Phi_{m}\right|^{2} \chi_{\nu_{0}, m} \\
& \leq e^{C\left(\tau-\tau_{0}\right)} \int(p-1) Q\left(\rho_{m}(0)\right)^{2} \chi_{v_{0}, m}+\left|\nabla \Phi_{m}(0)\right|^{2} \chi_{\nu_{0}, m} \\
& \quad+e^{C \tau} \int_{\tau_{0}}^{\tau} e^{-\left(C+2 \delta_{g}\right) \sigma} d \sigma \\
& \leq e^{C \Gamma}\left[C_{0} e^{-\delta_{g} \tau_{0}}+e^{-2 \delta_{g} \tau_{0}}\right] \leq 2 e^{C \Gamma} C_{0} e^{-\delta_{g} \tau_{0}}
\end{aligned}
$$

and hence

$$
\begin{aligned}
e^{\frac{4 \delta g}{5}} \tau & {\left[\int(p-1) Q \rho_{m}^{2} \chi_{v_{0}, m}+\left|\nabla \Phi_{m}\right|^{2} \chi_{\nu_{0}, m}\right] } \\
\leq & e^{\frac{4 \delta g}{5} \tau_{0}} e^{\frac{4 \delta g}{5} \Gamma}\left[\int(p-1) Q \rho_{m}^{2} \chi_{v_{0}, m}+\left|\nabla \Phi_{m}\right|^{2} \chi_{v_{0}, m}\right. \\
& \left.\times \int(p-1) Q \rho_{m}^{2} \chi_{\nu_{0}, m}+\left|\nabla \Phi_{m}\right|^{2} \chi_{v_{0}, m}\right] \\
= & 2 e^{C \Gamma} C_{0} e^{-\delta_{g} \tau_{0}} e^{\frac{4 \delta_{g}}{5} \tau_{0}} \leq e^{2 C \Gamma} e^{-\frac{\delta_{g}}{10} \tau_{0}} \leq 1,
\end{aligned}
$$

which concludes the proof of (8.23) and (8.15) for $\tau \in\left[\tau_{0}, \tau_{0}+\Gamma\right]$.

Step 3 Finite speed of propagation. We now pick a time $\tau_{f} \in\left[\tau_{0}+\Gamma, \tau^{*}\right]$ and propagate the bound (8.1) to the compact set $Z \leq Z_{0}$ using a finite speed of propagation argument. We claim:

$$
\|\rho\|_{H^{2 k_{0}\left(Z \leq \frac{Z_{0}}{2}\right)}}^{2}+\|\nabla \Psi\|_{H^{2 k_{0}\left(Z \leq \frac{Z_{0}}{2}\right)}}^{2} \leq C e^{-\delta_{g} \tau}
$$


Here the key is that (8.1) controls a norm on the set strictly including the light cone $Z \leq Z_{2}$. Let

$$
\hat{Z}_{a}=\frac{\tilde{Z}_{a}+Z_{2}}{2}
$$

and note that we may, without loss of generality by taking $a>0$ small enough, assume:

$$
\frac{\tilde{Z}_{a}}{\hat{Z}_{a}} \leq 2
$$

Recall that $\Gamma=\Gamma\left(Z_{0}\right)$ is parametrized by (8.22). We define

$$
\chi(\tau, Z)=\zeta\left(\frac{Z}{v(\tau)}\right), v(\tau)=\frac{Z_{0}}{2 \hat{Z}_{a}} e^{-\omega_{0}\left(\tau_{f}-\tau\right)}
$$

with $\omega_{0}>0$ defined in (8.21), (8.22) and a fixed spherically symmetric nonincreasing cut off function

$$
\zeta(Z)=\mid \begin{aligned}
& 1 \text { for } 0 \leq Z \leq \hat{Z}_{a} \\
& 0 \text { for } Z \geq \tilde{Z}_{a}
\end{aligned}, \quad \zeta^{\prime} \leq 0
$$

We define

$$
\tau_{\Gamma}=\tau_{f}-\Gamma
$$

so that from (8.22):

$$
\mid \begin{aligned}
& \tau_{0} \leq \tau_{\Gamma} \leq \tau^{*} \\
& v\left(\tau_{\Gamma}\right)=\frac{Z_{0}}{2 \hat{Z}_{a}} e^{-\omega_{0}\left(\tau_{f}-\tau_{\Gamma}\right)}=\frac{Z_{0}}{2 \hat{Z}_{a}} e^{-\omega_{0} \Gamma}=1 .
\end{aligned}
$$

We pick

$$
0 \leq m \leq 2 k_{0}
$$

then (8.26), (8.27) ensure $\operatorname{Supp}\left(\chi\left(\tau_{\Gamma}, \cdot\right)\right) \subset\left\{Z \leq \tilde{Z}_{a}\right\}$ and hence from (8.1):

$$
\left(\int(p-1) Q \rho_{m}^{2} \chi+\left|\nabla \Phi_{m}\right|^{2} \chi\right)\left(\tau_{\Gamma}\right) \lesssim e^{-\delta_{g} \tau_{\Gamma}} .
$$

This estimate implies that we can integrate energy identity (8.19) only on the interval $\left[\tau_{\Gamma}, \tau_{f}\right]$. We now estimate all terms in (8.19). 
Boundary terms. We compute the quadratic terms involving $\Lambda \chi$ which should be thought of as boundary terms. First

$$
\partial_{\tau} \chi(\tau, Z)=-\frac{\partial_{\tau} v}{v} \frac{Z}{v} \partial_{Z} \zeta\left(\frac{Z}{v}\right)=-\omega_{0} \Lambda \chi
$$

We now assume, recalling (8.16), that $\omega_{0}$ has been chosen small enough so that (8.21) holds, and hence the lower bound on the full boundary quadratic form using $\Lambda \chi \leq 0$ :

$$
\begin{aligned}
& \frac{1}{2} \int(p-1) Q \rho_{m}^{2}\left[\partial_{\tau} \chi+H_{2} \Lambda \chi\right] \\
& \quad+\frac{1}{2} \int\left|\nabla \Phi_{m}\right|^{2}\left[\partial_{\tau} \chi+H_{2} \Lambda \chi\right]+\int(p-1) Q \rho_{m} \nabla \chi \cdot \nabla \Phi_{m} \\
& =\int\left\{\frac{1}{2}(p-1) Q \rho_{m}^{2}\left[-\omega_{0}+H_{2}\right]\right. \\
& \left.\quad+\frac{1}{2}\left|\nabla \Phi_{m}\right|^{2}\left[-\omega_{0}+H_{2}\right]+(p-1) \frac{Q}{Z} \partial_{Z} \Phi_{m} \rho_{m}\right\} \Lambda \chi
\end{aligned}
$$

From (3.18), the discriminant of the above quadratic form is given by

$$
\begin{aligned}
& {\left[(p-1) \frac{Q}{Z}\right]^{2}-\left(-\omega_{0}+H_{2}\right)^{2}(p-1) Q} \\
& =(p-1) Q\left[\frac{(p-1) Q}{Z^{2}}-\left(-\omega_{0}+H_{2}\right)^{2}\right] \\
& =(p-1) \mu^{2} Q\left[\sigma^{2}-\left(-\frac{\omega_{0}}{\mu}+1-w\right)^{2}\right] \\
& =(p-1) \mu^{2} Q\left[-D(Z)+O\left(\omega_{0}\right)\right] .
\end{aligned}
$$

We then observe by definition of $\chi$ that for $\tau \geq \tau_{\Gamma}$ :

$$
Z \in \operatorname{Supp} \Lambda \chi \Leftrightarrow \hat{Z}_{a} \leq \frac{Z}{v(\tau)} \leq \tilde{Z}_{a} \Rightarrow Z \geq v(\tau) \hat{Z}_{a} \geq v\left(\tau_{\Gamma}\right) \hat{Z}_{a}=\hat{Z}_{a}
$$

from which since $\hat{Z}_{a}>Z_{2}$ :

$$
Z \in \operatorname{Supp} \Lambda \chi \Rightarrow-D(Z)+O\left(\omega_{0}\right)<0
$$

provided $0<\omega_{0} \ll 1$ has been chosen small enough. 
Together with (8.21) and $\Lambda \chi<0$, this ensures: $\forall \tau \in\left[\tau_{\Gamma}, \tau^{*}\right]$,

$$
\begin{aligned}
& \frac{1}{2} \int(p-1) Q \rho_{m}^{2}\left[\partial_{\tau} \chi+H_{2} \Lambda \chi\right] \\
& \quad+\frac{1}{2} \int\left|\nabla \Phi_{m}\right|^{2}\left[\partial_{\tau} \chi+H_{2} \Lambda \chi\right]+\int(p-1) Q \rho_{m} \nabla \chi \cdot \nabla \Phi_{m}<0
\end{aligned}
$$

Nonlinear terms. From (8.26), (8.25) for $\tau \leq \tau_{f}$ :

$\operatorname{Supp} \chi \subset\left\{Z \leq v(\tau) \tilde{Z}_{a}\right\} \subset\left\{Z \leq v\left(\tau_{f}\right) \tilde{Z}_{a}\right\}=\left\{Z \leq \frac{Z_{0}}{2} \frac{\tilde{Z}_{a}}{\hat{Z}_{a}}\right\} \subset\left\{Z \leq Z_{0}\right\}$, and hence from (8.14):

$$
\begin{aligned}
& \int \chi\left|\nabla \partial^{m} G_{\Phi}\right|^{2}+\int(p-1) Q \chi\left|\partial^{m} G_{\rho}\right|^{2} \\
& \lesssim\left\|\nabla G_{\Phi}\right\|_{H^{2 k_{0}\left(Z \leq Z_{0}\right)}}^{2}+\left\|G_{\rho}\right\|_{H^{2 k_{0}}\left(Z \leq Z_{0}\right)}^{2} \leq e^{-\frac{4 \delta g}{3} \tau} .
\end{aligned}
$$

Conclusion. Injecting the collection of above bounds into (8.19) and summing over $m \in\left[0,2 k_{0}\right]$ yields the crude bound: $\forall \tau \in\left[\tau_{\Gamma}, \tau_{f}\right]$,

$$
\begin{aligned}
& \frac{d}{d \tau}\left\{\sum_{m=0}^{2 k_{0}} \int(p-1) Q \rho_{m}^{2} \chi+\left|\nabla \Phi_{m}\right|^{2} \chi\right\} \\
& \leq C \sum_{m=0}^{2 k_{0}} \int(p-1) Q \rho_{m}^{2} \chi+\left|\nabla \Phi_{m}\right|^{2} \chi+e^{-\frac{4 \delta_{g}}{3} \tau} .
\end{aligned}
$$

We integrate the above on $\left[\tau_{\Gamma}, \tau_{f}\right]$ and conclude using

$$
\chi\left(\tau_{f}, Z\right)=\zeta\left(\frac{Z}{v\left(\tau_{f}\right)}\right)=\zeta\left(\frac{Z}{\frac{Z_{0}}{2 \hat{Z}_{a}}}\right)=1 \text { for } Z \leq Z_{0}
$$

and the initialization (8.28):

$$
\begin{aligned}
& {\left[\|\rho\|_{H^{2 k_{0}\left(Z \leq Z_{0}\right)}}^{2}+\|\nabla \Psi\|_{H^{2 k_{0}}\left(Z \leq Z_{0}\right)}^{2}\right]\left(\tau_{f}\right)} \\
& \quad \lesssim e^{C\left(\tau_{f}-\tau_{\Gamma}\right)} e^{-\delta_{g} \tau_{\Gamma}}+\int_{\tau_{\Gamma}}^{\tau_{f}} e^{C\left(\tau_{f}-\sigma\right)} e^{-\frac{4 \delta_{g}}{3} \sigma} d \sigma \\
& \quad \lesssim C(\Gamma) e^{-\delta_{g} \tau_{f}}=C\left(Z_{0}\right) e^{-\delta_{g} \tau_{f}} .
\end{aligned}
$$


Since the time $\tau_{f}$ is arbitrary in $\left[\tau_{0}+\Gamma, \tau^{*}\right]$, the bound (8.24) follows.

Step 4 Proof of (8.15). We run the energy identity (8.19) with $\chi_{\nu_{0}, m}$ and estimate each term.

Terms $\frac{Z_{0}}{3} \leq Z \leq \frac{Z_{0}}{2}$. In this zone, we have by construction

$$
\rho=\tilde{\rho}
$$

and hence the bootstrap bounds (4.38) imply

$$
\|\rho\|_{H^{k_{m}}\left(Z \leq \frac{Z_{0}}{2}\right)}+\|\nabla \Psi\|_{H^{k_{m}}\left(Z \leq \frac{Z_{0}}{2}\right)} \lesssim 1
$$

and hence interpolating with (8.24) for $k_{m}$ large enough:

$$
\begin{aligned}
\|\rho\|_{H^{m}\left(\frac{Z_{0}}{3} \leq Z \leq \frac{Z_{0}}{2}\right)} & \lesssim\|\rho\|_{H^{k_{m}}\left(\frac{Z_{0}}{3} \leq Z \leq \frac{Z_{0}}{2}\right)}^{\frac{m}{k^{2}}}\|\rho\|_{L^{2}\left(\frac{Z_{0}}{3} \leq Z \leq \frac{Z_{0}}{2}\right)}^{1-\frac{m}{2}} \lesssim e^{-\frac{\delta g}{2}\left(1-\frac{m}{k_{m}}\right)} \\
& \leq e^{-\frac{4 \delta_{g}}{10}}
\end{aligned}
$$

and similarly for the phase

$$
\|\nabla \Psi\|_{H^{m}\left(\frac{Z_{0}}{3} \leq Z \leq \frac{Z_{0}}{2}\right)} \lesssim e^{-\frac{\delta g}{2}\left(1-\frac{m}{k_{m}}\right)} \leq e^{-\frac{4 \delta g}{10}} .
$$

Linear term. We observe the cancellation using (8.17), (4.2):

$$
\begin{aligned}
\partial_{\tau} \chi_{v_{0}, m}+H_{2} \Lambda \chi_{v_{0}, m}= & \frac{1}{\langle Z\rangle^{d-2(r-1)+2\left(v_{0}-m\right)}}\left[-\mu \Lambda \zeta\left(\frac{Z}{Z^{*}}\right)\right] \\
& +\mu(1-w)\left[\frac{1}{\langle Z\rangle^{d-2(r-1)+2\left(v_{0}-m\right)}} \Lambda \zeta\left(\frac{Z}{Z^{*}}\right)\right. \\
& \left.+\Lambda\left(\frac{1}{\langle Z\rangle^{d-2(r-1)+2\left(v_{0}-m\right)}}\right) \zeta\left(\frac{Z}{Z^{*}}\right)\right] \\
= & -\mu\left[d-2(r-1)+2\left(v_{0}-m\right)\right] \chi_{\nu_{0}, m} \\
& +O\left(\frac{1}{\langle Z\rangle^{d-2(r-1)+2\left(v_{0}-m\right)+\omega}}\right)
\end{aligned}
$$

for some universal constant $\omega>0$. We now estimate the norm for $2 Z^{*} \leq Z \leq$ $3 Z^{*}$. Using spherical symmetry for $Z \geq 1$ and $m \geq 1$ :

$$
\left|Z^{m} \partial^{m} \rho\right| \lesssim \sum_{j=1}^{m} Z^{m} \frac{\left|\partial_{Z}^{j} \rho\right|}{Z^{m-j}} \lesssim \sum_{j=1}^{m} Z^{j}\left|\partial_{Z}^{j} \rho\right|
$$


and hence using the outer $L^{\infty}$ bound (4.40):

$$
\begin{aligned}
& \int_{2 Z^{*} \leq Z \leq 3 Z^{*}} \frac{(p-1) Q\left|\partial^{m} \rho\right|^{2}+\left|\partial^{m} \nabla \Phi\right|^{2}}{\langle Z\rangle^{d-2(r-1)+2\left(\nu_{0}-m\right)+\omega}} \\
& \lesssim \int_{2 Z^{*} \leq Z \leq 3 Z^{*}}\left[\sum_{j=0}^{m}\left|\frac{Z^{j} \partial_{Z}^{j} \rho}{\langle Z\rangle^{\frac{d}{2}+\nu_{0}+\frac{\omega}{2}}}\right|^{2}+\sum_{j=1}^{m+1}\left|\frac{Z^{j} \partial_{Z}^{j} \Phi}{\langle Z\rangle^{\nu_{0}+\frac{d}{2}-(r-1)+1+\frac{\omega}{2}}}\right|^{2}\right] \\
& \lesssim \int_{2 Z^{*} \leq Z \leq 3 Z^{*}}\left[\sum_{j=0}^{m}\left|\frac{Z^{j} \partial_{Z}^{j} \rho}{\rho_{P}\langle Z\rangle^{\frac{d}{2}+\nu_{0}+\frac{2(r-1)}{p-1}+\frac{\omega}{2}}}\right|^{2}\right. \\
& \quad+\sum_{j=1}^{m+1} \mid\langle Z\rangle^{r-2} \frac{Z^{j} \partial_{Z}^{j} \Psi}{\left.\left.\langle Z\rangle^{\nu_{0}+\frac{2(r-1)}{p-1}+\frac{d}{2}+\frac{\omega}{2}}\right|^{2}\right]} \\
& \lesssim \frac{1}{\left(Z^{*}\right)^{\omega+2\left[\nu_{0}+\frac{2(r-1)}{p-1}\right]}\left(1+b^{2}\left(Z^{*}\right)^{2(r-2)}\right) \leq e^{-\delta_{g} \tau}}
\end{aligned}
$$

using

$$
b\left(Z^{*}\right)^{r-2}=e^{\tau[-e+\mu(r-2)]}=e^{\tau[-e+1-2 \mu]}=1
$$

and the explicit choice from (4.17):

$$
2 \mu\left(v_{0}+\frac{2(r-1)}{p-1}\right)=\delta_{g} .
$$

Conclusion Injecting the above bounds into (8.19) yields:

$$
\begin{aligned}
\frac{1}{2} \frac{d}{d \tau}\left\{\int(p-1) Q \rho_{m}^{2} \chi_{\nu_{0}, m}+\left|\nabla \Phi_{m}\right|^{2} \chi_{\nu_{0}, m}\right\} \\
=-\int \chi_{\nu_{0}, m}\left[(p-1) Q \rho_{m}^{2}+\left|\nabla \Phi_{m}\right|^{2}\right]\left[\mu \nu_{0}+\frac{2 \mu(r-1)}{p-1}\right] \\
\quad+O\left(\int _ { Z _ { 0 } \leq Z \leq 2 Z ^ { * } } \chi _ { \nu _ { 0 } , m } \left[\sum_{m=0}^{m+1} \frac{\left|\partial_{Z}^{j} \Phi\right|^{2}}{\langle Z\rangle^{2(m+1-j)+2 \omega}}\right.\right. \\
\left.\left.\quad+\sum_{j=0}^{m} \frac{Q\left|\partial_{Z}^{j} \rho\right|^{2}}{\langle Z\rangle^{2(m-j)+2 \omega}}\right]+e^{-\frac{4 \delta_{g}}{5} \tau}\right) \\
\quad+O\left(\int \chi_{\nu_{0}, m}\left|\nabla \Phi_{m}\right|\left|\nabla \partial^{m} G_{\Phi}\right|+\int \chi_{\nu_{0}, m} Q\left|\rho_{m}\right|\left|\partial^{m} G_{\rho}\right|\right)
\end{aligned}
$$


and hence after summing over $m$ :

$$
\begin{aligned}
\frac{1}{2} & \frac{d}{d \tau}\left\{\sum_{m=0}^{2 k_{0}} \int(p-1) Q \rho_{m}^{2} \chi_{\nu_{0}, m}+\left|\nabla \Phi_{m}\right|^{2} \chi_{\nu_{0}, m}\right\} \\
= & -\mu\left[v_{0}+\frac{2(r-1)}{p-1}\right] \sum_{m=0}^{2 k_{0}} \int \chi_{\nu_{0}, m}\left[(p-1) Q \rho_{m}^{2}+\left|\nabla \Phi_{m}\right|^{2}\right] \\
& +O\left(e^{-\frac{4 \delta_{g}}{5} \tau}+\sum_{m=0}^{2 k_{0}} \int(p-1) Q \rho_{m}^{2} \chi_{\nu_{0}+\omega, m}+\left|\nabla \Phi_{m}\right|^{2} \chi_{\nu_{0}+\omega, m}\right) \\
& +\sum_{m=0}^{2 k_{0}} O\left(\int \chi_{\nu_{0}, m}\left|\nabla \Phi_{m}\right|\left|\nabla \partial^{m} G_{\Phi}\right|+\int \chi_{\nu_{0}, m} Q\left|\rho_{m}\right|\left|\partial^{m} G_{\rho}\right|\right)
\end{aligned}
$$

Using (8.24) we conclude

$$
\begin{aligned}
\frac{1}{2} & \frac{d}{d \tau}\left\{\sum_{m=0}^{2 k_{0}} \int(p-1) Q \rho_{m}^{2} \chi_{\nu_{0}, m}+\left|\nabla \Phi_{m}\right|^{2} \chi_{\nu_{0}, m}\right\} \\
= & -\mu\left[\nu_{0}+\frac{2(r-1)}{p-1}+O\left(\frac{1}{Z_{0}^{C}}\right)\right] \\
& \times \sum_{m=0}^{2 k_{0}} \int \chi_{v_{0}, m}\left[(p-1) Q \rho_{m}^{2}+\left|\nabla \Phi_{m}\right|^{2}\right] \\
& +O\left(e^{-\frac{4 \delta g}{5} \tau}+\sum_{m=0}^{2 k_{0}} \int \chi_{\nu_{0}, m}\left|\nabla \partial^{m} G_{\Phi}\right|^{2}\right. \\
& \left.+\int(p-1) Q \chi_{\nu_{0}, m}\left|\partial^{m} G_{\rho}\right|^{2}\right) .
\end{aligned}
$$

Therefore, using also (8.20), for $Z_{0}$ large enough and universal and

$$
2 \mu\left(v_{0}+\frac{2(r-1)}{p-1}\right)=\delta_{g}
$$

there holds

$$
\frac{d}{d \tau}\left\{\sum_{m=0}^{2 k_{0}} \int(p-1) Q \rho_{m}^{2} \chi_{v_{0}, m}+\left|\nabla \Phi_{m}\right|^{2} \chi_{v_{0}, m}\right\}
$$




$$
\leq-\frac{4 \delta_{g}}{5} \sum_{m=0}^{2 k_{0}} \int \chi_{v_{0}, m}\left[(p-1) Q \rho_{m}^{2}+\left|\nabla \Phi_{m}\right|^{2}\right]+C e^{-\frac{4 \delta_{g} \tau}{5}}
$$

Integrating in time and using (4.19) yields (8.15).

\subsection{Closing the bootstrap and proof of Theorem 1.1}

We are now in position to prove the bootstrap Proposition 4.4 which immediately implies Theorem 1.1.

Proof of Proposition 4.4 and Theorem 1.1 Recall that the non vanishing of the solution is ensured by (4.27). It remains to close the bound (4.26). Indeed, from (4.1), (4.2), (4.8) for $Z \geq Z^{*}$ :

$$
\frac{|\Delta u|}{\rho_{D}} \lesssim \frac{\left(Z^{*}\right)^{2}}{\rho_{D}}\left[\left|\Delta \rho_{\mathrm{Tot}}\right|+\frac{\left|\partial_{Z} \rho_{\mathrm{Tot}}\right|\left|\partial_{Z} \Psi_{\mathrm{Tot}}\right|}{b}+\frac{\left|\rho_{\mathrm{Tot}} \Delta \Psi_{\mathrm{Tot}}\right|}{b}\right] \lesssim 1
$$

where we used (4.40) in the last step. The $|u|^{p}$ term is handled similarily, and (4.26) is improved for $b_{0}$ small enough. ${ }^{14}$ Note also that the bounds (4.40) imply

$$
\|u(t)\|_{H^{k_{c}}} \leq C(t)
$$

for $\frac{d}{2} \ll k_{c} \ll k_{m}$ for times in the bootstrap interval and hence the bootstrap time is strictly smaller than the life time provided by standard Cauchy theory. We now conclude from a classical topological argument à la Brouwer. The bounds of Sects. 5, 6, 7, 8 have been shown to hold for all initial data on the time interval $\left[\tau_{0}, \tau_{0}+\Gamma\right]$ with $\Gamma$ large. Moreover, as explained in the proof of Lemma 8.1, they can be immediately propagated to any time $\tau^{*}$ after a choice of projection of initial data on the subspace of unstable modes $\mathbb{P} X\left(\tau_{0}\right)$.

This is done as follows. We define a decomposition of the the set of initial data $X\left(\tau_{0}\right)$. Recall that the restriction of the data $X\left(\tau_{0}\right)$ to the interval $\left[0, Z_{a}\right]$ is contained in the Sobolev space $\mathbb{H}_{2 k_{0}}$ which can be split into a direct sum of the stable and unstable subspaces

$$
\mathbb{H}_{2 k_{0}}=U \bigoplus V
$$

\footnotetext{
14 The smallness of $b_{0}$ is responsible for the size of the time length between initial data and formation of a singularity.
} 
For functions defined for all $Z$, we define a subspace of functions satisfying the assumptions of Sect. 4.3 on initial data

$$
V_{\text {reg }}=\left\{(\tilde{\rho}, \Psi):\|\tilde{\rho}, \Psi\|_{k_{m}}<\infty\right\}
$$

with the property that $V_{\text {reg }}$ has the same dimension as $V$ and its restriction to $\left[0, Z_{a}\right]$ satisfies the property

$$
\operatorname{dist}\left(V_{\text {reg }}, V\right)_{\mathbb{H}_{2 k_{0}}}<e^{-3 \delta_{g} \tau_{0}} .
$$

Note that the space $V_{\text {reg }}$ consists of functions which are defined for all $Z$ and which are more regular on $\left[0, Z_{a}\right]$ than the ones contained in $V$. We can explicitly construct $V_{\text {reg }}$ by defining it as the linear space generated by $\left\{v_{r e g}^{1}, \ldots, v_{r e g}^{n}\right\}$ where each $v_{r e g}^{j}$ is obtained from the element $v^{j}$-a generator of $V$-by a smoothing and an extension procedure. The precise details of both the smoothing and extension are not important, as long as (8.36) is ensured to hold. By (8.36), the projection $\mathbb{P}$ (composed with the restriction to $\left[0, Z_{a}\right]$ ) is an isomorphism between $V_{\text {reg }}$ and $V$. Denoting the inverse of this isomorphism by $\mathbb{I}$, we see that it satisfies the property that $\mathbb{P} \circ \mathbb{I}$ is the identity map on $V$. We also define a complementary subspace $W$ such that the space of all data with $\|\tilde{\rho}, \Psi\|_{k_{m}}<\infty$ decomposes into the sum

$$
V_{\text {reg }} \bigoplus W
$$

with $W$ obeying the additional property that $\mathbb{P} W=0$. We can further restrict $W$ to consist of functions satisfying all of the assumptions of Sect. 4.3 on initial data. Let $X(\tau)$ be the solution of the nonlinear problem (3.14) with the initial data $X\left(\tau_{0}\right)$. We now apply Lemma 3.5 to $x(\tau)=\mathbb{P} X(\tau)$. We choose the initial data $X\left(\tau_{0}\right)$ obeying all the initial data bounds with the additional condition that it is of the form $w+\mathbb{I} v$ with a fixed element $w \in W$ obeying the bound

$$
\|w\|_{\mathbb{H}_{2 k_{0}}} \leq e^{-\frac{\delta g}{2} \tau_{0}}
$$

and any element $v \in V$ obeying

$$
\|v\|_{\mathbb{H}_{2 k_{0}}} \leq e^{-\frac{3 \delta g}{5} \tau_{0}}
$$

For such initial data $\mathbb{P} X\left(\tau_{0}\right)=v$ and the solution $X(\tau)$ depends continuously on $v$. As a consequence, the right hand side $F$ of Lemma 3.5, which represents the projection $\mathbb{P}$ of the nonlinear terms $G$ in (3.14), can be shown to depend continuously on $x(\tau)$. All other assumptions of Lemma 3.5 follow from the 
bounds of Sects. 5, 6, 7, 8 and Lemma 8.1. It now follows from Lemma 3.5 that, for any $w$ fixed as above, there exists $v^{*}=v^{*}(w)$ such that the exit time $\tau^{*}$ corresponding to the initial data $X\left(\tau_{0}\right)=\mathbb{I} v^{*}+w$ satisfies $\tau^{*}=\infty$. This concludes the proof of Theorem 1.1.

Acknowledgements P.R. is supported by the ERC-2014-CoG 646650 SingWave. P.R. would like to thank the Université de la Côte d'Azur where part of this work was done for its kind hospitality, and N. Burq (Orsay) and L. Vega (Bilbao) for stimulating discussions at very early stages of this work. I.R. is partially supported by the NSF Grant DMS \#1709270 and a Simons Investigator Award. J.S. is supported by the ERC Grant ERC-2016 CoG 725589 EPGR. The authors would also like to thank the anonymous referees for their careful reading and numerous valuable suggestions and comments.

Open Access This article is licensed under a Creative Commons Attribution 4.0 International License, which permits use, sharing, adaptation, distribution and reproduction in any medium or format, as long as you give appropriate credit to the original author(s) and the source, provide a link to the Creative Commons licence, and indicate if changes were made. The images or other third party material in this article are included in the article's Creative Commons licence, unless indicated otherwise in a credit line to the material. If material is not included in the article's Creative Commons licence and your intended use is not permitted by statutory regulation or exceeds the permitted use, you will need to obtain permission directly from the copyright holder. To view a copy of this licence, visit http://creativecommons.org/licenses/by/4.0/.

\section{Appendix A: Comparison with compressible Euler dynamics}

We consider the compressible Euler equations with a polytropic equation of state:

$$
\mid \begin{aligned}
& \partial_{t} \rho+\nabla \cdot(\rho u)=0, \\
& \rho \partial_{t} u+\rho u \cdot \nabla u+\nabla P=0, \quad x \in \mathbb{R}^{d} \\
& P=\frac{\gamma-1}{\gamma} \rho^{\gamma}
\end{aligned}
$$

for $\gamma>1$.

Each step below should be compared with the corresponding step in Sect. 2.1.

Step 1 Scaling and renormalization. The scaling symmetry ${ }^{15}$ is

$$
\lambda^{\frac{2}{\gamma+1}} \rho\left(\lambda^{\frac{2 \gamma}{\gamma+1}} t, \lambda x\right), \quad \lambda^{\frac{\gamma-1}{\gamma+1}} u\left(\lambda^{\frac{2 \gamma}{\gamma+1}} t, \lambda x\right) .
$$

We renormalize self-similarly

$$
\frac{d \tau}{d t}=\frac{1}{\lambda \frac{2 \gamma}{\gamma+1}}, \quad-\frac{\lambda_{\tau}}{\lambda}=\frac{1}{2}
$$

15 We choose a 1-parameter of scaling transformation, which is compatible with the NavierStokes equations, out of a larger 2-parameter family of possible transformations. 
and obtain

$$
\mid \begin{aligned}
& \partial_{\tau} \rho+\frac{1}{2}\left(\frac{2}{\gamma+1} \rho+y \cdot \nabla \rho\right)+\nabla \cdot(\rho u)=0 \\
& \rho \partial_{\tau} u+\frac{1}{2} \rho\left(\frac{\gamma-1}{\gamma+1} u+y \cdot \nabla u\right)+\rho u \cdot \nabla u+\nabla P=0 .
\end{aligned}
$$

As we did for the Schrödinger equations in Lemma 2.1, we proceed with a front renormalization

$$
\rho \mapsto \frac{1}{b^{\frac{1}{\gamma-1}}} \rho(y \sqrt{b}), \quad u \mapsto \frac{1}{b^{\frac{1}{2}}} u(y \sqrt{b})
$$

with

$$
\frac{b_{\tau}}{b}=-e
$$

and consider a potential spherically symmetric flow with $u=\nabla \Psi=\Psi^{\prime}$. Note that for the Euler equations, unlike the Schrödinger and Navier-Stokes cases, the front renormalization corresponds to a symmetry of the equations. A direct computation in which we also integrate the second equation leads to

$$
\mid \begin{aligned}
& -\partial_{\tau} \rho=\Delta \Psi+\left(\frac{e}{\gamma-1}+\frac{1}{\gamma+1}\right)+\frac{\nabla \rho}{\rho} \cdot\left[\left(\frac{1-e}{2}\right) Z+\nabla \Psi\right] \\
& -\partial_{\tau} \Psi=\frac{1}{2}|\nabla \Psi|^{2}+\left(e-\frac{1}{\gamma+1}\right) \Psi-1+\left(\frac{1-e}{2}\right) Z \cdot \nabla \Psi+\rho^{\gamma-1}
\end{aligned}
$$

These equations should be directly compared to the Schrödinger equations in their front renormalized, self-similar, hydrodynamical formulation (2.5).

A stationary solution of the above equation satisfies

$$
\mid \begin{aligned}
& \Delta \Psi+\left(\frac{e}{\gamma-1}+\frac{1}{\gamma+1}\right)+\frac{\nabla \rho}{\rho} \cdot\left[\left(\frac{1-e}{2}\right) Z+\nabla \Psi\right]=0, \\
& \frac{1}{2}|\nabla \Psi|^{2}+\left(e-\frac{1}{\gamma+1}\right) \Psi+\left(\frac{1-e}{2}\right) Z \cdot \nabla \Psi+\rho^{\gamma-1}=1 .
\end{aligned}
$$

Step 2 Emden transform. We introduce the variables

$$
V=\Psi^{\prime}, \quad S=\sqrt{2} \rho^{\frac{\gamma-1}{2}}
$$

where $S$ is the space dependent sound speed, so that equivalently taking the derivative of the second equation:

$$
\mid \begin{aligned}
& V^{\prime}+\frac{d-1}{Z} V+\left(\frac{e}{\gamma-1}+\frac{1}{\gamma+1}\right)+\frac{2}{\gamma-1} \frac{S^{\prime}}{S}\left[\left(\frac{1-e}{2}\right) Z+V\right]=0 \\
& V V^{\prime}+\left(e-\frac{1}{\gamma+1}\right) V+\left(\frac{1-e}{2}\right)\left(Z V^{\prime}+V\right)+S S^{\prime}=0 .
\end{aligned}
$$


Let

$$
\varkappa=\log Z, \quad V(Z)=v(\varkappa), \quad S(Z)=s(\varkappa), \quad Z \frac{d}{d Z}=\frac{d}{d \varkappa} .
$$

First equation

$$
\frac{v^{\prime}}{Z}+\frac{(d-1) v}{Z}+\left(\frac{e}{\gamma-1}+\frac{1}{\gamma+1}\right)+\frac{2}{\gamma-1} \frac{s^{\prime}}{s} \frac{1}{Z}\left[\left(\frac{1-e}{2}\right) Z+V\right]
$$

and hence letting

$$
v(\varkappa)=e^{\varkappa} w, \quad s(\varkappa)=e^{\varkappa} \sigma
$$

yields

$$
\begin{aligned}
& \left(w^{\prime}+w\right)+(d-1) w \\
& +\left(\frac{e}{\gamma-1}+\frac{1}{\gamma+1}\right)+\frac{2}{\gamma-1}\left(\frac{\sigma^{\prime}}{\sigma}+1\right)\left(\frac{1-e}{2}+w\right)=0
\end{aligned}
$$

i.e.,

$$
\sigma w^{\prime}+\frac{2}{\gamma-1}\left(\frac{1-e}{2}+w\right) \sigma^{\prime}+\sigma\left[\left(d+\frac{2}{\gamma-1}\right) w+\frac{2 \gamma}{\gamma^{2}-1}\right]=0 .
$$

Second equation. We get

$$
\frac{v v^{\prime}}{Z}+\left(e-\frac{1}{\gamma+1}\right) v+\left(\frac{1-e}{2}\right)\left(v^{\prime}+v\right)+\frac{s s^{\prime}}{Z}=0
$$

and hence

$$
w\left(w^{\prime}+w\right)+\left(e-\frac{1}{\gamma+1}\right) w+\left(\frac{1-e}{2}\right)\left(w^{\prime}+2 w\right)+\sigma\left(\sigma^{\prime}+\sigma\right)=0
$$

or equivalently

$$
\left(w+\frac{1-e}{2}\right) w^{\prime}+\sigma \sigma^{\prime}+\left(w^{2}+\frac{\gamma}{\gamma+1} w+\sigma^{2}\right)=0
$$

We have obtained:

Lemma A.1 (Emden transform) Let

$$
\varkappa=\log Z, \quad \Psi^{\prime}(Z)=e^{\varkappa} w(\varkappa), \quad S(Z)=e^{\varkappa} \sigma(\varkappa), \quad S=\sqrt{2} \rho^{\frac{\gamma-1}{2}},
$$


then

$$
\mid \begin{aligned}
& \left(w+\frac{1-e}{2}\right) w^{\prime}+\sigma \sigma^{\prime}+\left(w^{2}+\frac{\gamma}{\gamma+1} w+\sigma^{2}\right)=0 \\
& \sigma w^{\prime}+\frac{2}{\gamma-1}\left(\frac{1-e}{2}+w\right) \sigma^{\prime}+\sigma\left[\left(d+\frac{2}{\gamma-1}\right) w+\frac{2 \gamma}{\gamma^{2}-1}\right]=0
\end{aligned}
$$

Step 3 Renormalized form. We define

$$
\ell=\frac{2}{\gamma-1}, \quad r=\frac{2 \gamma}{(1-e)(\gamma+1)}, \quad \phi^{2}\left(\frac{2}{e-1}\right)^{2}=\ell
$$

and the renormalized unknowns

$$
U=\frac{2}{c-1} w, \quad \Sigma=\frac{\sigma}{\phi} .
$$

The second equation becomes:

$$
\begin{aligned}
& \Sigma \frac{e-1}{2} U^{\prime}+\ell \frac{e-1}{2}(U-1) \Sigma^{\prime} \\
& +\Sigma \ell\left[\left(1+\frac{d}{\ell}\right) \frac{e-1}{2} U+\frac{2 \gamma}{\left(\gamma^{2}-1\right) \ell}\right]=0
\end{aligned}
$$

i.e.,

$$
\frac{\sigma}{\ell} U^{\prime}+(U-1) \sigma^{\prime}+\sigma\left[\left(1+\frac{d}{\ell}\right) U-\frac{2 \gamma}{(1-e)(\gamma+1)}\right]=0 .
$$

The first equation becomes

$$
\begin{aligned}
& \left(\frac{e-1}{2}\right)^{2} U U^{\prime}+\phi^{2} \Sigma \Sigma^{\prime} \\
& +\left[\left(\frac{e-1}{2}\right)^{2} U^{2}+\frac{\gamma}{\gamma+1} \frac{e-1}{2} U+\phi^{2} \Sigma^{2}\right]=0
\end{aligned}
$$

and hence (A.5) yields:

$$
(U-1) U^{\prime}+\ell \Sigma \Sigma^{\prime}+\left[U^{2}-\frac{2 \gamma}{(1-e)(\gamma+1)} U+\ell \Sigma^{2}\right]=0 .
$$

We arrive at the renormalized system

$$
\mid \begin{aligned}
& (U-1) U^{\prime}+\ell \Sigma \Sigma^{\prime}+\left(U^{2}-r U+\ell \Sigma^{2}\right)=0 \\
& \frac{\Sigma}{\ell} U^{\prime}+(U-1) \Sigma^{\prime}+\Sigma\left[U\left(\frac{d}{\ell}+1\right)-r\right]=0
\end{aligned}
$$


which is identical to the system (2.11) for the defocusing NLS but with the parameters

$$
\ell=\frac{2}{\gamma-1}, \quad r=\frac{2 \gamma}{(1-e)(\gamma+1)}
$$

in place of

$$
\ell=\frac{4}{p-1}, \quad r=\frac{2}{(1-e)}
$$

\section{Appendix B: Hardy inequality}

Lemma B.1 Assume $2 \gamma \notin \mathbb{Z}$. Then, for all $u \in C_{\mathrm{rad}}^{\infty}(r \geq 1)$ and $j \geq 1$ :

$$
\int_{r \geq 1} r^{2 \gamma} u^{2} d r \lesssim j, \gamma\|u\|_{H^{j}(1 \leq r \leq 2)}^{2}+\int_{r \geq 1} r^{2(\gamma+j)}\left|\partial_{r}^{j} u\right|^{2} d r
$$

Proof Assume $2 \gamma \neq-1$, We integrate by parts

$$
\begin{aligned}
& \int_{r \geq 1} r^{2 \gamma} u^{2} d r=\frac{1}{2 \gamma+1}\left[r^{2 \gamma+1} u^{2}\right]_{1}^{+\infty}-\frac{2}{2 \gamma+1} \int_{r \geq 1} r^{2 \gamma+1} u \partial_{r} u d r \\
& \leq C\|u\|_{H^{1}(1 \leq r \leq 2)}^{2}+C\left(\int_{r \geq 1} r^{2 \gamma} u^{2} d r\right)^{\frac{1}{2}}\left(\int_{r \geq 1} r^{2 \gamma+2}\left(\partial_{r} u\right)^{2} d r\right)^{\frac{1}{2}},
\end{aligned}
$$

where we used the one dimensional Sobolev embedding, and (B.1) for $j=1$ follows by Hölder. For higher values of $j$, (B.1) now follows by induction.

\section{Appendix C: Commutator for $\Delta^{k}$}

Lemma C.1 (Commutator for $\Delta^{k}$ ) Let $k \geq 1$, then for any two smooth functions $V, \Phi$, there holds:

$$
\left[\Delta^{k}, V\right] \Phi-2 k \nabla V \cdot \nabla \Delta^{k-1} \Phi=\sum_{|\alpha|+|\beta|=2 k,|\beta| \leq 2 k-2} c_{k, \alpha, \beta} \partial^{\alpha} V \partial^{\beta} \Phi .(\mathrm{C} .1)
$$

where $\partial^{\alpha}=\partial_{1}^{\alpha_{1}} \cdots \partial_{d}^{\alpha_{d}},|\alpha|=\alpha_{1}+\cdots+\alpha_{d}$.

Proof We argue by induction on $k$. For $k=1$ :

$$
\Delta(V \Phi)-V \Delta \Phi=2 \nabla V \cdot \nabla \Phi .
$$


We assume (C.1) for $k$ and prove $k+1$. Indeed,

$$
\begin{aligned}
\Delta^{k+1}(V \Phi)=\Delta\left(\left[\Delta^{k}, V\right] \Phi+V \Delta^{k} \Phi\right) \\
=\Delta\left(2 k \nabla V \cdot \nabla \Delta^{k-1} \Phi+\sum_{|\alpha|+|\beta|=2 k,|\beta| \leq 2 k-2} c_{k, \alpha, \beta} \partial^{\alpha} V \partial^{\beta} \Phi+V \Delta^{k} \Phi\right) \\
=2 k \nabla V \cdot \nabla \Delta^{k} \Phi \quad \tilde{c}_{k, \alpha, \beta} \partial^{\alpha} V \partial^{\beta} \Phi+V \Delta^{k+1} \Phi+2 \nabla V \cdot \nabla \Delta^{k} \Phi \\
\quad+\sum_{|\alpha|+|\beta|=2 k+2,|\alpha| \geq 2} \sum=V \Delta^{k+1} \Phi+2(k+1) \nabla V \cdot \nabla \Delta^{k} \Phi+\sum_{|\alpha|+|\beta|=2 k+2,|\alpha| \geq 1} c_{k+1, \alpha, \beta} \partial^{\alpha} V \partial^{\beta} \Phi
\end{aligned}
$$

and (C.1) is proved.

\section{Appendix D: Behaviour of Sobolev norms}

We compute Sobolev norms assuming that the leading part of the solution is given by (1.9). Computations below are formal but could be justified as a consequence of the bootstrap estimates.

Dirichlet energy of the profile. We show that the Dirichlet energy of the solution remains bounded until blow up time. Indeed, we recall (1.8), (1.9) and compute:

$$
\|\nabla u\|_{L^{2}}^{2} \sim 1+\|\nabla u\|_{L^{2}(|x| \leq 1)}^{2}=1+\int_{|x| \leq 1}|\nabla \rho|^{2} d x+\int_{|x| \leq 1} \rho^{2}|\nabla \phi|^{2} .
$$

We compute for the first term:

$$
\begin{aligned}
& \int_{|x| \leq 1}|\nabla \rho|^{2} d x \sim \frac{1}{\left(T_{*}-t\right)^{\frac{4(r-1)}{r(p-1)}}} \int_{|Z| \leq \frac{1}{(T-t)^{\frac{1}{r}}}} \frac{\left(T_{*}-t\right)^{\frac{d}{r}}}{\left(T_{*}-t\right)^{\frac{2}{r}}} \frac{Z^{d-1} d Z}{\langle Z\rangle^{\frac{4(r-1)}{p-1}+2}} \\
& =\frac{1}{\left(T_{*}-t\right)^{\frac{2}{r}\left(\frac{2(r-2)}{p-1}+\frac{2}{p-1}-\frac{d}{2}+1\right)}} \int_{|Z| \leq \frac{1}{\left(T_{*}-t\right)^{\frac{1}{r}}}} \frac{d Z}{Z^{1+2\left(1+\frac{2(r-2)}{p-1}+\frac{2}{p-1}-\frac{d}{2}\right)}} \\
& =\frac{1}{\left(T_{*}-t\right)^{\frac{2}{r}(1-\sigma)}} \int_{|Z| \leq \frac{1}{\left(T_{*}-t\right)^{\frac{1}{r}}}} \frac{d Z}{\langle Z\rangle^{1+2(1-\sigma)}}
\end{aligned}
$$

with

$$
\sigma=s_{c}-\frac{2(r-2)}{p-1}>1 \Leftrightarrow \frac{d}{2}-\frac{\ell}{2}-\frac{\ell}{2}(r-2)>1 \Leftrightarrow d-\ell(r-1)>2
$$




$$
\begin{aligned}
& \Leftrightarrow d-2>\ell\left(\frac{\ell+d}{\ell+\sqrt{d}}-1\right)=\frac{\ell(d-\sqrt{d})}{\ell+\sqrt{d}} \\
& \Leftrightarrow(d-2) \sqrt{d}+\ell(\sqrt{d}-2)>0
\end{aligned}
$$

which holds, and hence

$$
\int_{|x| \leq 1}|\nabla \rho|^{2} d x \lesssim 1
$$

Similarily:

$$
\begin{aligned}
& \int_{|x| \leq 1} \rho^{2}|\nabla \phi|^{2} \\
& =\frac{1}{\left(T_{*}-t\right)^{\frac{4(r-1)}{r(p-1)}+\frac{2(r-2)}{r}}} \int_{|Z| \leq \frac{1}{\left(T_{*}-t\right) \frac{1}{r}}} \frac{\left(T_{*}-t\right)^{\frac{d}{r}}}{\left(T_{*}-t\right)^{\frac{2}{r}}} \frac{Z^{d-1} d Z}{\langle Z\rangle^{2(r-2+1)+\frac{4(r-1)}{p-1}}} \\
& =\frac{1}{\left(T_{*}-t\right)^{\frac{2}{r}\left(r-2+\frac{2(r-2)}{p-1}+1+\frac{2}{p-1}-\frac{d}{2}\right)}} \int_{|Z| \leq \frac{1}{\left(T_{*}-t\right)^{\frac{1}{r}}}} \frac{d Z}{\langle Z\rangle^{1+2\left(1+r-2+\frac{2(r-2)}{p-1}+\frac{2}{p-1}-\frac{d}{2}\right)}}
\end{aligned}
$$

and at $r^{*}(\ell)$ :

$$
\begin{aligned}
& r-2+\frac{2(r-2)}{p-1}+1+\frac{2}{p-1}-\frac{d}{2}<0 \Leftrightarrow(r-1)\left(1+\frac{\ell}{2}\right)<\frac{d}{2} \\
& \Leftrightarrow(2+\ell)(d-\sqrt{d})<d(\ell+\sqrt{d}) \Leftrightarrow d(\sqrt{d}-2)+(\ell+2) \sqrt{d}>0
\end{aligned}
$$

which holds and hence

$$
\int_{|x| \leq 1} \rho^{2}|\nabla \phi|^{2} \lesssim 1 .
$$

In view of the above, we infer

$$
\sup _{t \in\left[0, T_{*}\right)}\|\nabla u(t, \cdot)\|_{L^{2}}^{2} \lesssim 1 .
$$

Blow up of high enough Sobolev norms below the scaling. Let us now compute the behavior of higher Sobolev norms. We unfold the change of variables

$$
\mid \begin{aligned}
& u(t, x)=\frac{1}{\lambda(t)^{\frac{2}{p-1}}} v(s, y) e^{i \gamma}, \quad y=\frac{x}{\lambda} \\
& v(s, y)=\frac{1}{(\sqrt{b})^{\frac{2}{p-1}}}\left(\rho_{\mathrm{Tot}} e^{\frac{i}{b}} \Psi_{\mathrm{Tot}}\right)(\tau, Z), \quad Z=y \sqrt{b}
\end{aligned}
$$


which yields

$$
\begin{aligned}
& \left\|\nabla^{s} u\right\|_{L^{2}}=\frac{1}{\lambda^{s-s_{c}}}\left\|\nabla^{s} v\right\|_{L^{2}} \\
& \geq \frac{1}{\lambda^{s-s_{c}}(\sqrt{b})^{\frac{2}{p-1}}}(\sqrt{b})^{s-\frac{d}{2}}\left\|\nabla^{s}\left(\rho_{\operatorname{Tot}} e^{\frac{i \Psi_{\mathrm{Tot}}}{b}}\right)\right\|_{L^{2}(|Z| \geq 1)} \\
& \gtrsim e^{\frac{s-s_{C}}{2} \tau}(\sqrt{b})^{s-\frac{d}{2}-\frac{2}{p-1}}\left\|\nabla^{s}\left(\rho_{\operatorname{Tot}} e^{\frac{\Psi_{\text {Tot }}}{b}}\right)\right\|_{L^{2}(|Z| \leq 1)} \\
& \gtrsim e^{\frac{s-s_{C}}{2} \tau}(\sqrt{b})^{s-\frac{d}{2}-\frac{2}{p-1}} \frac{1}{b^{s}} \\
& \gtrsim e^{\tau\left[\frac{s-s_{C}}{2}+\frac{e}{2}\left(s+\frac{d}{2}+\frac{2}{p-1}\right)\right]}
\end{aligned}
$$

which blows up as soon as

$$
s>\sigma=\frac{1}{1+e}\left[s_{c}-e\left(\frac{d}{2}+\frac{2}{p-1}\right)\right] .
$$

We can check that at $r^{*}(\ell)$ :

$$
\begin{aligned}
\sigma>1 & \Leftrightarrow \frac{d}{2}-\frac{2}{p-1}>1+e+e\left(\frac{d}{2}+\frac{2}{p-1}\right) \\
& \Leftrightarrow \frac{d}{2}(1-e)>(1+e)\left(1+\frac{\ell}{2}\right) \\
& \Leftrightarrow \frac{d}{2} \frac{2}{r}>\frac{1}{r}(r-1)(\ell+2) \Leftrightarrow d>(\ell+2)\left(\frac{d+\ell}{\ell+\sqrt{d}}-1\right) \\
& \Leftrightarrow d(\sqrt{d}-2)+(\ell+2) \sqrt{d}>0
\end{aligned}
$$

The last inequality holds for our assumptions on $d \geq 5$ and $\ell>0$.

\section{References}

1. Alazard, T., Carles, R.: Loss of regularity for supercritical nonlinear Schrödinger equations. Math. Ann. 343, 397-420 (2009)

2. Bahouri, H., Gérard, P.: High frequency approximation of solutions to critical nonlinear wave equations. Am. J. Math. 121(1), 131-175 (1999)

3. Banica, V., Vega, L.: On the stability of a singular vortex dynamics. Commun. Math. Phys. 286(2), 593-627 (2009)

4. Beceanu, M., Deng, Q., Soffer, A., Wu, Y.: Large global solutions for nonlinear Schrödinger equations III, energy-supercritical cases. arXiv:1901.07709 [math.AP]

5. Bizon, P., Biernat, P.: Generic self-similar blowup for equivariant wave maps and YangMills fields in higher dimensions. Commun. Math. Phys. 338(3), 1443-1450 (2015)

6. Bourgain, J.: Problems in Hamiltonian PDE'S. Geom. Funct. Anal. (2000). Special volume Vision in Mathematics, pp. 32-56 
7. Bourgain, J.: Global well posedness of defocusing critical nonlinear Schrödinger equations in the radial case. J. Am. Math. Soc. 12(1), 145-171 (1999)

8. Bricmont, J., Kupiainen, A.: Renormalization group and asymptotics of solutions of nonlinear parabolic equations. Commun. Pure Appl. Math. 47, 893-922 (1994)

9. Cazenave, T., Naumkin, I.: Modified scattering for the critical nonlinear Schrödinger equation. J. Funct. Anal. 274, 402-432 (2018)

10. Cazenave, T., Weissler, F.: The Cauchy problem for the critical nonlinear Schrödinger equation. Non. Anal. TMA 14, 807-836 (1990)

11. Colliander, J., Keel, M., Staffilani, G., Takaoka, H., Tao, T.: Global well-posedness and scattering for the energy-critical nonlinear Schrödinger equation in $\mathbb{R}^{3}$. Ann. Math. (2) 167(3), 767-865 (2008)

12. Colliander, J., Simpson, G., Sulem, C.: Numerical simulations of the energy- supercritical nonlinear Schrödinger equation. J. Hyp. Differ. Eqs. 7(2), 279-296 (2010)

13. Colombo, M., Haffter, S.: Global regularity for the nonlinear wave equation with slightly supercritical power. arXiv:1911.02599

14. Collot, C.: Type II blow up manifolds for the energy supercritical semilinear wave equation. Mem. Am. Math. Soc. 252(1205), (2018)

15. Collot, C., Raphaël, P., Szeftel, J.: On the stability of type I blow up for the energy super critical heat equation. Mem. Am. Math. Soc. 260(1255), (2019)

16. Collot, C., Merle, F., Raphaël, P.: On strongly anisotropic type II blow up. Preprint (2017)

17. Dafermos, M., Rodnianski, I.: Lectures on black holes and linear waves. Clay Math. Proc. 17:97-205. American Mathematicals Society, Providence, RI (2013)

18. Donninger, R.: On stable self-similar blowup for equivariant wave maps. Commun. Pure Appl. Math. 64(8), 1095-1147 (2011)

19. Donninger, R., Schörkhuber, B.: On blowup in supercritical wave equations. Commun. Math. Phys. 346(3), 907-943 (2016)

20. Engel, K.J., Nagel, R.: One Parameter Semi Groups for Linear Evolution Equations. Springer

21. Gallay, T., Wayne, C.E.: Invariant manifolds and the long-time asymptotics of the NavierStokes and vorticity equations on $\mathbb{R}^{2}$. Arch. Ration. Mech. Anal. 163(3), 209-258 (2002)

22. Ginibre, J., Velo, G.: On a class of nonlinear Schrödinger equations. I. The Cauchy problem, general case. J. Funct. Anal. 32(1), 1-32 (1979)

23. Ginibre, J., Velo, G.: Scattering theory in the energy space for a class of nonlinear Schrödinger equations. J. Math. Pure Appl. 64, 363-401 (1985)

24. Grenier, E.: Semiclassical limit of the nonlinear Schrödinger equation in small time. Proc. Am. Math. Soc. 126(2), 523-530 (1998)

25. Grillakis, G.: On nonlinear Schrödinger equations. CPDE 25, 1827-1844 (2000)

26. Guderley, G.: Starke kugelige und zylindrische verdichtungsstöfle in der nähe des kugelmittelpunktes bzw. der zylinderachse. Luftahrtforschung 19, 302 (1942)

27. Hayashi, N., Nakamitsu, K., Tsutsumi, M.: Nonlinear Schrödinger equations in weighted Sobolev spaces. Funkcial Ekvac. 31, 363-381 (1988)

28. Herrero, M.A., Velázquez, J.J.L.: Explosion de solutions des équations paraboliques semilinéaires supercritiques. C. R. Acad. Sci. Paris 319, 141-145 (1994)

29. Jin, S., Levermore, C., McLaughlin, D.: The semiclassical limit of the defocusing NLS hierarchy. Commun. Pure Appl. Math. 52(5), 613-654 (1999)

30. Kenig, C.E., Merle, F.: Global well-posedness, scattering and blow-up for the energycritical, focusing, non-linear Schrödinger equation in the radial case. Invent. Math. 166(3), 645-675 (2006)

31. Kenig, C.E., Merle, F.: Nondispersive radial solutions to energy supercritical non-linear wave equations, with applications. Am. J. Math. 133(4), 1029-1065 (2011)

32. Killip, R., Visan, M.: Energy supercritical NLS: critical $\dot{H}^{s}$ bounds imply scattering. Commun. PDE 35(6), 945-987 (2010) 
33. Krieger, J., Schlag, W.: Large global solutions for energy supercritical nonlinear wave equations on $\mathbb{R}^{3+1}$. J. Anal. Math. 133, 91-131 (2017)

34. Krieger, J., Schlag, W., Tataru, D.: Renormalization and blow up for charge one equivariant critical wave maps. Invent. Math. 171(3), 543-615 (2008)

35. Lebeau, G.: Perte de régularité pour les équations d'condes sur-critiques. Bull. Soc. Math. France 133(1), 145-157 (2005)

36. Lepin, L.A.: Self-similar solutions of a semilinear heat equation. Mat. Model. 2, 63-7 (1990)

37. Madelung, E.: Quantentheorie in hydrodynamischer Form. Z. Phys. 40(34), 322-326 (1927)

38. Matano, H., Merle, F.: On nonexistence of type II blowup for a supercritical nonlinear heat equation. Commun. Pure Appl. Math. 57(11), 1494-1541 (2004)

39. Matano, H., Merle, F.: Classification of type I and type II behaviors for a supercritical nonlinear heat equation. J. Funct. Anal. 256(4), 992-1064 (2009)

40. Merle, F., Raphaël, P.: Profiles and quantization of the blow up mass for critical nonlinear Schrödinger equation. Commun. Math. Phys. 253(3), 675-704 (2005)

41. Merle, F., Raphaël, P., Rodnianski, I.: Blowup dynamics for smooth data equivariant solutions to the critical Schrödinger map problem. Invent. Math. 193(2), 249-365 (2013)

42. Merle, F., Raphaël, P., Rodnianski, I.: Type II blow up for the energy supercritical NLS. Camb. J. Math. 3(4), 439-617 (2015)

43. Merle, F., Raphaël, P., Rodnianski, I., Szeftel, J.: On smooth self similar solutions to the compressible Euler equation. Preprint (2019)

44. Merle, F., Raphaël, P., Rodnianski, I., Szeftel, J.: On the implosion of a viscous compressible gas. Preprint (2019)

45. Merle, F., Raphaël, P., Szeftel, J.: On strongly anisotropic type I blow up. To appear in Int. Math. Res. Not

46. Merle, F., Vega, L.: Compactness at blow-up time for $L^{2}$ solutions of the critical nonlinear Schrödinger equation in 2D. Int. Math. Res. Notices 8, 399-425 (1998)

47. Merle, F., Zaag, H.: Stability of the blow-up profile for equations of the type $u_{t}=\Delta u+$ $|u|^{p-1} u$. Duke Math. J. 86(1), 143-195 (1997)

48. Merle, F., Zaag, H.: Existence and universality of the blow-up profile for the semilinear wave equation in one space dimension. J. Funct. Anal. 253(1), 43-121 (2007)

49. Mizoguchi, N.: Rate of type II blowup for a semilinear heat equation. Math. Ann. 339(4), 839-877 (2007)

50. Murphy, J., Zhang, Y.: Numerical simulations for the energy-supercritical nonlinear wave equation. arXiv:1905.10446 [math.NA]

51. Perelman, G.: Blow up dynamics for equivariant critical Schrödinger maps. Commun. Math. Phys. 330(1), 69-105 (2014)

52. Raphaël, P., Rodnianski, I.: Stable blow up dynamics for the critical co-rotational wave maps and equivariant Yang-Mills problems. Publ. Math. Inst. Hautes Etudes Sci. 115, $1-122$ (2012)

53. Raphaël, P., Schweyer, R.: Stable blowup dynamics for the 1-corotational energy critical harmonic heat flow. Commun. Pure Appl. Math. 66(3), 414-480 (2013)

54. Rodnianski, I., Sterbenz, J.: On the formation of singularities in the critical $O$ (3) $\sigma$-model. Ann. Math. 172(1), 187-242 (2010)

55. Ryckman, E., Visan, M.: bGlobal well-posedness and scattering for the defocusing energycritical nonlinear Schrödinger equation in $\mathbb{R}^{1+4}$. Am. J. Math. 129, 1-60 (2007)

56. Schmudgen, K.: Unbounded Self Adjoint Operators on Hilbert Spaces. Springer

57. Sedov, L.I.: Similarity and Dimensional Methods in Mechanics. Academic Press, New York (1959)

58. Shatah, J.: Weak solutions and development of singularities of the $S U$ (2) $\sigma$-model. Commun. Pure Appl. Math. 41(4), 459-469 (1988) 
59. Struwe, M.: Global well-posedness of the Cauchy problem for a super-critical nonlinear wave equation in two space dimensions. Math. Ann. 350(3), 707-719 (2011)

60. Tao, T.: Global well-posedness and scattering for the higher-dimensional energy-critical non-linear Schrödinger equation for radial data. N. Y. J. Math. 11, 57-80 (2005)

61. Tao, T.: Finite-time blowup for a supercritical defocusing nonlinear wave system. Anal. PDE 9(8), 1999-2030 (2016)

62. Tao, T.: Finite time blowup for a supercritical defocusing nonlinear Schrödinger system. Anal. PDE 11(2), 383-438 (2018)

63. Tao, T.: Global regularity for a logarithmically supercritical defocusing nonlinear wave equation for spherically symmetric data. J. Hyperbolic Differ. Equ. 4(2), 259-265 (2007)

64. Thomann, L.: Instabilities for supercritical Schrödinger equations in analytic manifolds. J. Differ. Equ. 245(1), 249-280 (2008)

65. Visan, M.: The defocusing energy-critical nonlinear Schrödinger equation in higher dimensions. Duke Math. J. 138(2), 281-374 (2007)

Publisher's Note Springer Nature remains neutral with regard to jurisdictional claims in published maps and institutional affiliations. 